\title{
Revisión de los criterios de discriminación florística de las alisedas riparias templadas (sub)litorales del extremo noroccidental ibérico
}

Recibido: 3 xaneiro 2018 / Aceptado: 15 marzo 2018

(C) Universidade de Santiago de Compostela 2018

\begin{abstract}
Resumen Se revisan los criterios florísticos de diferenciación de las alisedas riparias presentes en los territorios litorales y sublitorales del extremo NW Ibérico a partir de la comparación de la información disponible en trabajos previos (197 inventarios) con la aportada en 288 nuevas muestras obtenidas mediante la aplicación de la metodología fitosociológica sigmatista. Aunque se mantienen las tres asociaciones vegetales descritas hasta el momento, se proponen nuevos grupos de especies diferenciales para remarcar su separación florística y se precisan sus límites biogeográficos. Además, se propone una nueva ordenación sintaxonómica para estos bosques por debajo del nivel de asociación (subasociaciones, variantes, subvariantes y facies) dentro del esquema fitosociológico seguido.
\end{abstract}

Palabras clave Bosques de ribera - NW Ibérico - Taxones diferenciales - Sintaxonomía.

Reassessment of the floristic differences between north-west iberian temperate (sub)littoral riparian blackalder forests

Abstract In this paper we reassess the floristic groups used until now to differentiate the riparian black alder-forests existing in the litoral and sublittoral cantabro-atlantic territories of the extreme NW Iberian Peninsula. For this purpose, we compared the available floristic information about these forests (197 relevés) to a database of 288 new relevés collected following the sigmatistic phytosociological

Manuel Antonio Rodríguez Guitián · Rosa Romero Franco Depto. de Produción Vexetal e Proxectos de Enxeñaría. EPS de Lugo. USC. Campus Universitario s/n. 27002-Lugo. Gl-1934 Territorio \& Biodiversidade Correo-e: manuelantonio.rodriguez@usc.es

Pablo Ramil-Rego · Javier Ferreiro da Costa Gl-1934 Territorio \& Biodiversidade

Instituto de Biodiversidade Agraria e Desenvolvemento Rural. USC. Campus Universitario s/n. 27002-Lugo. method. As a result, new sets of vascular plants are proposed to differentiate the three associations considered whose biogeographical boundaries are redelimited too. In addition, a new syntaxonomical scheme for lower ranks below association level (subassociations, variants, subvariants and facies) is introduced.

Key words Back-alder riparian woodlands - NW Spain . Differential taxa $\cdot$ Syntaxonomy.

\section{Introducción y objetivos}

Los bosques de ribera dominados por el aliso (Alnus glutinosa Gaert.) son ecosistemas de un elevado valor ecológico cuya protección se considera prioritaria dentro del ámbito de la Unión Europea, tal y como se desprende de su inclusión dentro del Anexo I de la DC 92/43/CEE bajo el código 91E0*. Tanto su extensión como su estado de conservación se encuentran amenazados debido a la reducción de su área original de presencia, así como por la introducción en el medio de diferentes agentes microbianos (hongos del género Phythopthora) que afectan, en muchos casos de manera letal, a su especie arbórea característica (Hansen \& Sutton 2000, Haque \& Díez 2012, Ramil-Rego \& Ferreiro da Costa 2016). En este escenario, el conocimiento de la variabilidad florística de estos bosques a escala continental y regional resulta determinante para que el mantenimiento y mejora de su estado de conservación, en aplicación de la Directiva antes mencionada, tenga lugar sin menoscabo de las peculiaridades botánicas existentes a lo largo del amplio territorio en el que dichos bosques están presentes (Rodríguez González 2008; Biurrun et al. 2014, 2016).

Aunque desde mediados del siglo pasado se venían empleando algunas propuestas nomenclaturales y de distribución de los tipos de bosques de ribera existentes en el área litoral-sublitoral del extremo noroccidental ibérico (Braun-Blanquet et al. 1956, Casaseca 1959, Bellot 1968, Dalda 1972), el trabajo publicado por Amigo et al. (1987) sobre las alisedas riparias cántabro-atlánticas constituye la 
aportación fitosociológica fundamental en lo que al conocimiento de este tipo de bosques se refiere. No en vano, esta propuesta ha sido utilizada como referencia básica en multitud de estudios posteriores, cuyo ámbito varía desde caracterizaciones a nivel local, comarcal o regional (Izco et al. 1998, Díaz González \& Fernández Prieto 1994, Rivas-Martínez et al. 2001, 2002; Rodríguez Guitián 2004, 2005, 2010a; Lara et al. 2004, Mayor \& Fernández 2007, Rodríguez Guitián et al. 2014, Biurrun et al. 2014, 2016) hasta revisiones a nivel ibérico o europeo (European Communities 1991, Devillers \& DevillersTerschuren 1996, Davies \& Moss 2002, Biurrun et al. 2016, Douda et al. 2016). En el trabajo de Amigo et al. (1987) se establecen tres tipos principales de alisedas riparias (asociaciones) a lo largo de los territorios eurosiberianos comprendidos entre la cuenca del Río Asón (Cantabria) y la desembocadura del Río Miño: cántabro-euskaldunas (Hyperico androsaemi-Alnetum glutinosae), cantábricas occidentales y orocantábricas occidentales (Valeriano pyrenaicae-Alnetum glutinosae) y galaico-portuguesas (Senecioni bayonnensis-Alnetum glutinosae), cuyas fronteras naturales se situarían, aproximadamente, en la desembocadura del Río Nalón, en el caso de las dos primeras, y el estuario del Río Eume, para las dos últimas.

Si bien en el trabajo comentado se establecen una serie de especies discriminantes entre los tipos de bosques descritos (Tabla 1), tanto en sus tablas de inventarios originales como en otras publicadas posteriormente, así como en nuestras observaciones de campo, se pone en evidencia la existencia de tramos fluviales dentro del ámbito geográfico citado cuyas alisedas de ribera presentan una composición florística que no se ajusta claramente a ninguna de las combinaciones de especies típicas de las asociaciones propuestas. Esta "indefinición florística" se concreta en dos situaciones: a) la aparición conjunta de especies que "a priori" se establecieron como excluyentes ("especies diferenciales") entre las tres asociaciones citadas; y b) la ausencia de determinadas especies que se habrían considerado como de presencia constante o casi ("especies características"). Estas combinaciones florísticas de difícil interpretación fitosociológica se dan mayoritariamente a lo largo del tramo comprendido entre las desembocaduras de los ríos Masma (Lugo) y Mandeo (A Coruña), área dentro de la que el citado estudio de Amigo et al. (1987) aporta un escaso número de inventarios, pero también aparecen en los territorios próximos a la desembocadura del Río Nalón (Asturias). Ello nos ha motivado a profundizar en el conocimiento de la variabilidad florística de las alisedas riparias en el extremo occidental de la Cornisa Cantábrica, con el ánimo de establecer las causas que determinan la "indefinición florística" comentada y proponer criterios alternativos que faciliten su encuadre fitosociológico, aspectos que constituyen el objetivo fundamental del presente estudio.

\begin{tabular}{|c|c|c|c|c|}
\hline & \multicolumn{3}{|c|}{ Asociaciones } \\
\hline \multirow{3}{*}{ 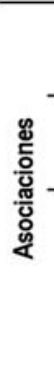 } & $S b-A g$ & -.. & $\begin{array}{l}\text { Galium broterianum, Fraxinus angustifolia (p.p), } \\
\text { Fraxinus } x \text { oxycarpa (p.p) }\end{array}$ & $\begin{array}{l}\text { Carex elata subsp, reuteriana, Fraxinus } \\
\text { angustifolia, Fraxinus x oxycarpa, Galium } \\
\text { broterianum, Osmunda regalis (p.p.) }\end{array}$ \\
\hline & $V p-A g$ & $\begin{array}{l}\text { Carex remota, Carex sylvatica, Circaea } \\
\text { lutetiana, Festuca gigantea, Stachys sylvatica, } \\
\text { Ulmus glabra, Woodwardia radicans }\end{array}$ & ... & $\begin{array}{l}\text { Carex elata subsp, reuteriana, Valeriana } \\
\text { pyrenaica, Fraxinus angustifolia (p.p.), } \\
\text { Osmunda regalis (p.p.), Salix salviffolia (p.p.) }\end{array}$ \\
\hline & $\mathrm{Ha}-\mathrm{Ag}$ & $\begin{array}{l}\text { Carex remota, Carex sylvatica, Circaea } \\
\text { lutetiana, Equisetum telmateia, Festuca } \\
\text { gigantea, Helleborus viridis subsp. occidentalis, } \\
\text { Lamiastrum galeobdolon, Saxifraga hirsuta, } \\
\text { Stachys sylvatica, Stegnogramma pozoi, Ulmus } \\
\text { glabra, Woodwardia radicans }\end{array}$ & $\begin{array}{l}\text { Equisetum telmateia, Helleborus viridis subsp. } \\
\text { occidentalis, Lamiastrum galeobdolon, } \\
\text { Saxifraga hirsuta, Stegnogramma pozoi }\end{array}$ & $\cdots$ \\
\hline
\end{tabular}

Tabla 1.- Grupos de especies exclusivas (diferenciales) propuestos para la discriminación florística de las tres asociaciones de alisedas riparias cántabro-atlánticas descritas por Amigo et al. (1987). Acrónimos: Sb-Ag: Senecioni bayonnensis-Alnetum glutinosae; Vp-Ag: Valeriano pyrenaicae-Alnetum glutinosae; Ha-Ag: Hyperico androsaemi-Alnetum glutinosae

\section{Material y métodos}

Para la realización del presente trabajo se han recogido inventarios florísticos a lo largo de diversas cuencas fluviales del extremo NW ibérico comprendidas entre la desembocadura de los ríos Nalón y Miño (Figura 1). En la toma de datos se ha seguido la metodología fitosociológica sigmatista (Braun-Blanquet 1979), idéntica a la empleada en el trabajo de referencia de Amigo et al. (1987) y de la mayoría de trabajos posteriores, empleándose en la nomenclatura botánica las propuestas para los géneros contenidos en los volúmenes disponibles de Flora Iberica (Castroviejo 1986-2013, Talavera et al. 2014, Devesa et al.
2015, Muñoz et al. 2015), y en los de Flora Europaea (Tutin et al. 1964-1980) para el resto. En los aspectos sintaxonómicos se sigue básicamente el esquema propuesto recientemente por Mucina et al. (2016) y los grupos de taxones característicos de unidades sintaxonómicas de rango superior propuestos por Biurrun et al. (2016).

Con la información recogida acerca de la composición florística de los bosques estudiados se han confeccionado tablas florísticas que han sido comparadas con la información disponible en trabajos previos (Braun-Blanquet et al. 1958, Casaseca 1959, Bellot 1968, Díaz 1975, Amigo 
1984, Amigo et al. 1987, Pulgar 1990, Silva-Pando 1990, Romero Buján 1993, Mayor \& Fernández 2007, Rodríguez Guitián et al. 2014, López Castro et al. 2015), en su mayor parte accesibles desde el portal electrónico del Sistema de Información de la Vegetación Ibérica y Macaronésica (SIVIM, http://www.sivim.info/sivi/).

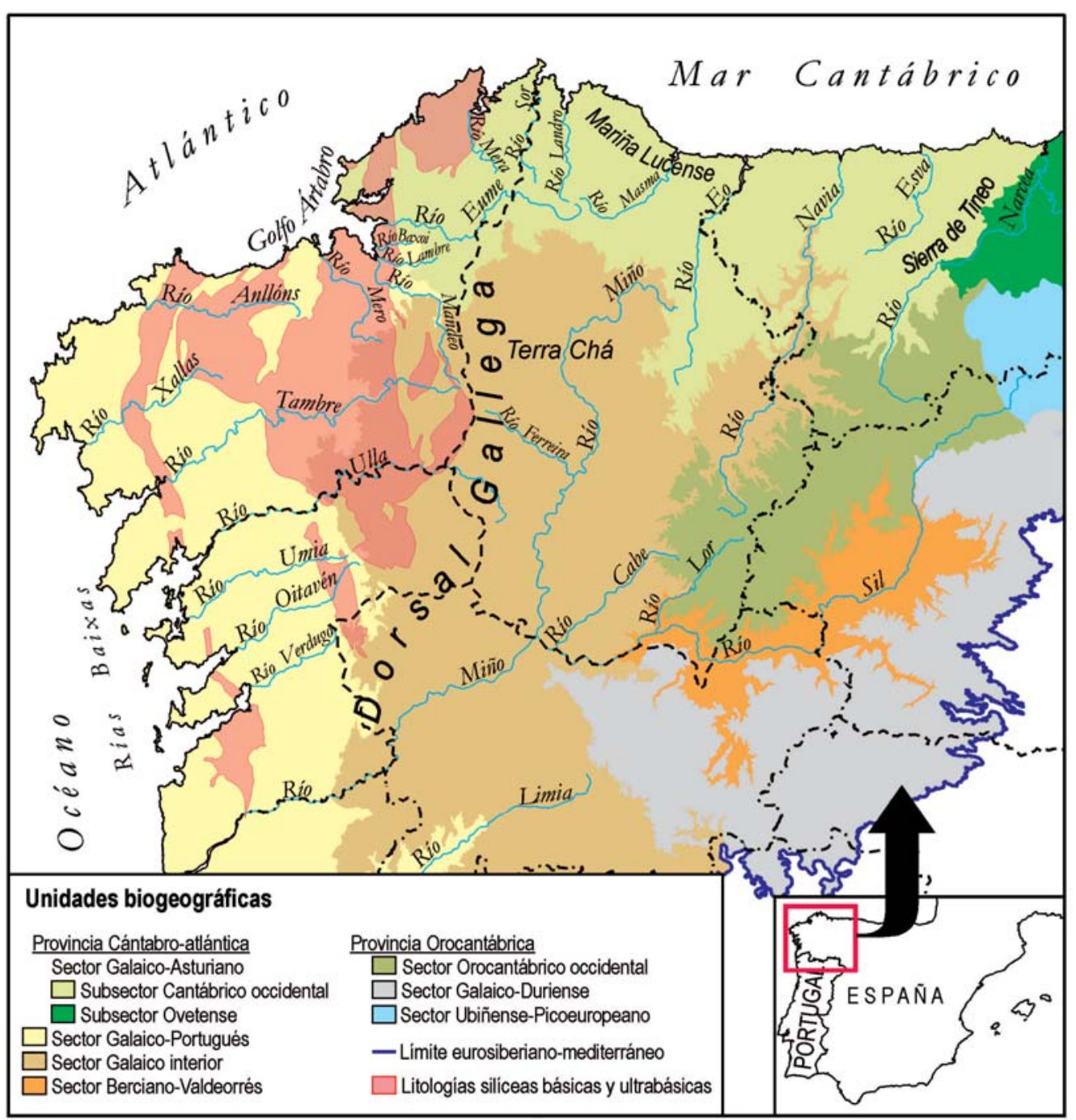

Figura 1.- Localización del área de estudio con indicación de las unidades biogeográficas y diversos accidentes geográficos citados en el texto. Unidades biogeográficas adaptadas de Rodríguez Guitián \& Ramil Rego (2008)

\section{Resultados y discusión}

A lo largo de los trabajos de campo efectuados se han recogido 288 nuevos inventarios florísticos de bosques de ribera dominados por el aliso (Figura 2; Anexo, tablas I a XIV) que han sido comparados con un total de 197 inventarios de procedencia bibliográfica. Para facilitar la interpretación fitosociológica realizada se ha construido una tabla sintética (Tabla 2) en la que se señalan las principales diferencias florísticas existentes entre los tipos de bosques estudiados. No obstante, antes de entrar en la presentación y discusión propiamente dicha de los resultados obtenidos en este trabajo, queremos realizar algunos comentarios acerca de diversos aspectos cualitativos y cuantitativos de la información que sobre estos bosques han aportado previamente otros autores.

Consideraciones sobre las características de la información de referencia

En trabajos anteriores (Rodríguez Guitián 2006, 2010a, 2010b) hemos discutido la importancia que tiene la elección apropiada de la unidad de muestreo y la época del año para la realización de los inventarios florísticos en la 
caracterización de las comunidades vegetales. La primera de estas cuestiones resulta de primordial importancia a la hora de determinar las especies que, por su mayor constancia en el medio ecológico estudiado, se consideran características de una comunidad en el sentido fitosociológico, así como aquellas otras que, por su ausencia en otros medios comparables, se consideran diferenciales frente a aquellos. Si la unidad de muestreo no alcanza una superficie mínima representativa de la frecuencia y abundancia con la que aparecen en la naturaleza las especies que en ella se integran, la separación de una determinada comunidad de otras semejantes teniendo como referencia un conjunto de inventarios de calidad "deficiente" puede ser ardua y, además, de difícil correspondencia con los fragmentos de dicha comunidad que pueden encontrarse en el campo (Dengler et al. 2009).

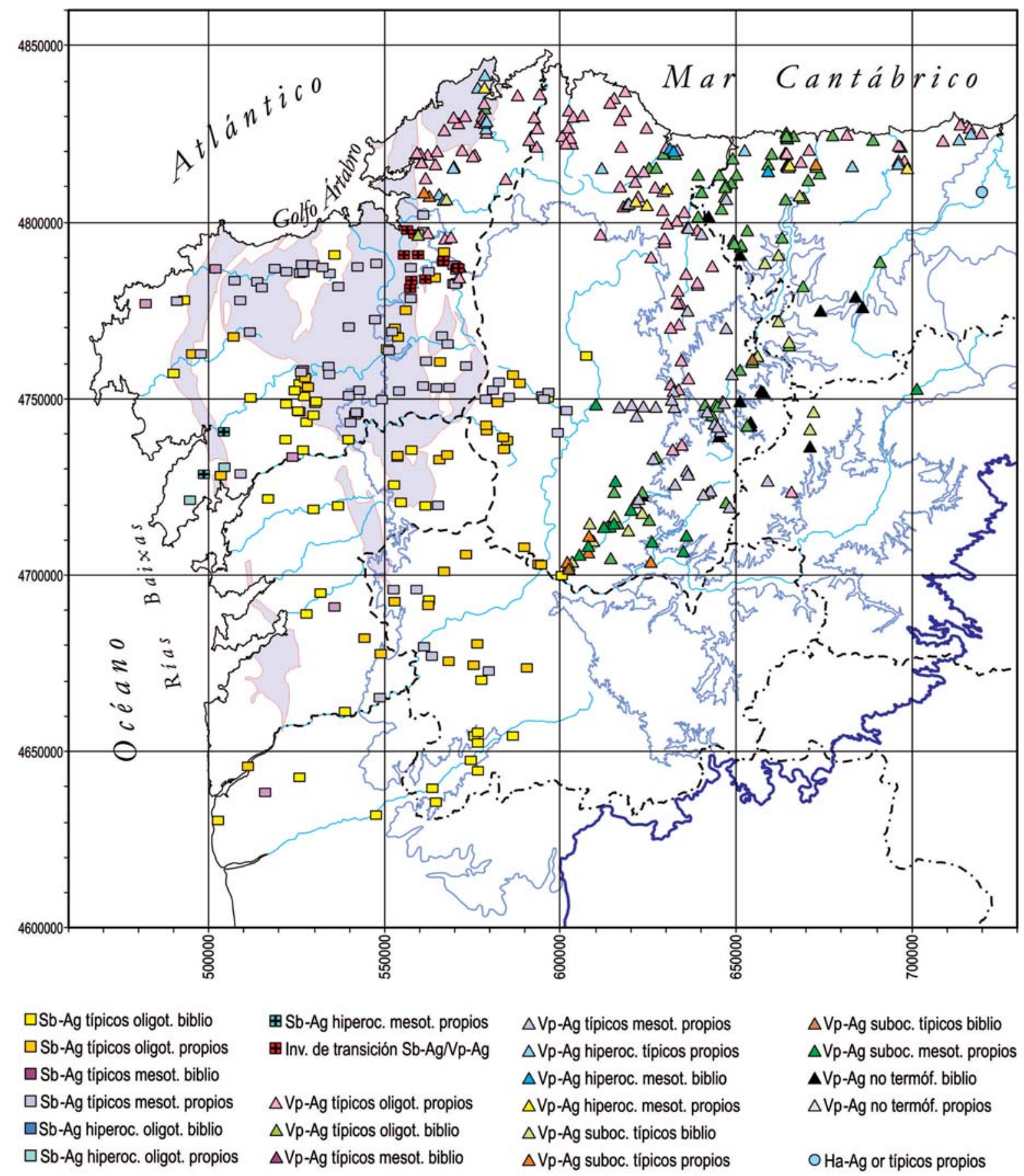

Figura 2.- Localización de los inventarios utilizados en este trabajo. En sombreado gris se delimitan los afloramientos de rocas metamórficas silíceas básicas y ultrabásicas 
Tabla 2.- Tabla sintética (frecuencia de aparición de taxones en \%) de alisedas riparias litorales y sublitorales cántabro-atlánticas agrupadas según los criterios florísticos mostrados en la Figura 6. Por razones de espacio, se ha prescindido de los taxones arbóreos de "E1+E2" y de "E3: Otros taxones" presentes en menos de seis columnas. En las columnas formadas por menos de 4 inventarios se muestra la frecuencia absoluta de aparición de los diferentes taxones presentes. Se destacan en negrita las frecuencias superiores al $50 \%$. ${ }^{*}=$ taxón adventicio. Procedencia de los inventarios: Pr: inventarios propios; Bib: inventarios bibliográficos. * $=$ taxón naturalizado.

\begin{tabular}{|c|c|c|c|c|c|c|c|c|c|c|c|c|c|c|c|c|c|c|c|c|c|c|c|c|}
\hline \multirow{2}{*}{$\begin{array}{l}\text { Asociación } \\
\text { Subasociación }\end{array}$} & \multicolumn{8}{|c|}{ Senecioni-Alnetum } & \multicolumn{13}{|c|}{ Valeriano-Alnetum } & \multicolumn{3}{|c|}{ Hyperico-Al } \\
\hline & $t$ & $t$ & $\mathrm{t}$ & $\mathrm{t}$ & $\mathrm{t}$ & $\tau$ & $t$ & $t$ & $t$ & $t$ & $\mathrm{t}$ & $t$ & $t$ & l & $t$ & $\mathrm{t}$ & & t & t & nt? & & or & or & \\
\hline ariante & $t$ & $t$ & $t$ & $t$ & $l$ & ho & ho & ho & $t$ & $t$ & $t$ & $t$ & ho & ho & ho & sho $s$ & sho & sho & sho & $t$ & $t$ & $t$ & $t$ & ho \\
\hline ubvariante & $t$ & $t$ & $\mathrm{~m}$ & $\mathrm{~m}$ & $\mathrm{~m}$ & $t$ & $t$ & $\mathrm{~m}$ & $t$ & $t$ & $\mathrm{~m}$ & $\mathrm{~m}$ & $t$ & $t$ & $\mathrm{~m}$ & $t$ & $t$ & $t$ & $\mathrm{~m}$ & $t$ & $t$ & $t$ & $t$ & $t$ \\
\hline acies & $t$ & $t$ & $t$ & $t$ & cont. & $\mathrm{t}$ & $t$ & $t$ & $t$ & $t$ & $t$ & $t$ & $t$ & $t$ & $t$ & $t$ & $\mathrm{t}$ & $t$ & $t$ & $t$ & $t$ & $t$ & $\mathrm{t}$ & $t$ \\
\hline ocedencia inventarios & $\mathrm{Pr}$ & $\mathrm{Bib}$ & $\mathrm{Pr}$ & $\mathrm{Bib}$ & $\operatorname{Pr}$ & $\mathrm{Pr}$ & Bib & $\operatorname{Pr}$ & $\mathrm{Pr}$ & Bib & $\operatorname{Pr}$ & $\mathrm{Bib}$ & $\mathrm{Pr}$ & B & $\mathrm{Pr}$ & $\mathrm{Pr}$ & Bib & $\mathrm{Pr}$ & Bib & $\mathrm{Pr}$ & Bib & $\mathrm{Pr}$ & Bib & Bib \\
\hline$d$ & 37 & 54 & 66 & 7 & 10 & 2 & 2 & 2 & 96 & 68 & 24 & 10 & 14 & 3 & 6 & 7 & 18 & 12 & 3 & 11 & 15 & 1 & 14 & 3 \\
\hline náx.) & 600 & 300 & 6001 & $150 !$ & 500 & 300 & -- & 300 & 500 & 400 & 500 & 400 & 500 & 600 & 400 & 6002 & 200 & 600 & 400 & 300 & 300 & --- & 4002 & 250 \\
\hline omedio) & 352 & 103 & 381 & 68 & 355 & 300 & --- & 270 & 325 & 110 & 308 & 170 & 287 & 383 & 292 & 457 & 121 & 433 & 210 & 231 & 101 & 400 & 164 & 183 \\
\hline$p$ & 100 & 10 & 200 & 40 & 200 & 300 & --- & 240 & 100 & 20 & 100 & 20 & 100 & 150 & 200 & 300 & 70 & 300 & 80 & 100 & 20 & --- & 40 & 100 \\
\hline & 71 & 59 & 81 & 34 & 81 & 49 & 63 & 46 & 87 & 66 & 89 & 79 & 93 & 82 & 63 & 97 & 38 & 111 & 78 & 53 & 70 & --- & 54 & 54 \\
\hline io) & 48 & 25 & 53 & 26 & 64 & 47 & 61 & 44 & 52 & 23 & 55 & 42 & 51 & 59 & 51 & 71 & $29 !$ & 70 & 46 & 40 & 25 & 59 & 36 & 48 \\
\hline to & 31 & 7 & 35 & 17 & 37 & 45 & 58 & 41 & 22 & 7 & 25 & 17 & 27 & 32 & 39 & 47 & 11 & 51 & 24 & 31 & 12 & -- & 14 & 43 \\
\hline & $1 a$ & $1 b$ & $2 a$ & $2 b$ & 3 & $4 a$ & $4 b$ & 5 & $6 a$ & $6 \mathrm{~b}$ & $7 a$ & $7 \mathrm{~b}$ & $8 a$ & $8 b$ & 9 & $10 \mathrm{a} 1$ & $10 \mathrm{~b}$ & $11 \mathrm{a}$ & $11 b$ & $12 a$ & $12 b$ & 13 & $14 \mathrm{a}$ & $14 b$ \\
\hline \multicolumn{25}{|l|}{$(>4,0 \mathrm{~m})+\mathrm{E}_{2}(>1,5-4,0 \mathrm{~m}$} \\
\hline nus glutinosa & 100 & 100 & 1001 & 100 & 100 & 2 & 2 & 2 & 100 & 100 & 100 & 100 & 100 & 3 & 100 & 100 & 100 & 100 & 3 & 100 & 100 & 4 & 100 & 100 \\
\hline & 94,6 & 90,7 & 92,4 & 100 & 90,0 & 2 & 2 & 2 & 87,5 & 67,6 & 83,3 & 0,0 & 71,4 & 3 & 3,3 & 100 & $\pi$ & 91,7 & 3 & 6 & 66,7 & 1 & & 33,3 \\
\hline $7 a$ & 29,7 & 13,0 & 54,52 & 28,6 & 100 & 1 & 1 & 1 & 76,0 & 26,5 & 79,2 & 50,0 & 100 & 3 & 100 & 71,42 & $27, \varepsilon$ & 83,3 & 1 & 63,66 & 66,7 & $1:$ & & 100 \\
\hline & 10,8 & 11,1 & 10,61 & 14,3 & 30,0 & - & 1 & - & 51,0 & 29,4 & 66,7 & 70,0 & 57,1 & 1 & 66,7 & 57,15 & $50,($ & 91,7 & 3 & 54,5 & 46,7 & 3 & 7,13 & 33,3 \\
\hline & 24,33 & 35,2 & 39,44 & 42,9 & 70,0 & 1 & - & - & 40,6 & 10,3 & 41,7 & 40,0 & 57,1 & 1 & 16,7 & 85,72 & 27,8 & 83,3 & 3 & 27,31 & 313,3 & & 35,76 & 66,7 \\
\hline & 89,23 & 37,0 & 90,9 & 14,3 & 90,0 & 1 & - & 2 & 56,3 & 4,4 & 33,3 & & 50,0 & 1 & 16,7 & 85,72 & 22,2 & 75,0 & 2 & 63,6 & 6,7 & + & 50,0 & \\
\hline & 78,4 & 64,8 & 66 , & 57,1 & 40,0 & 2 & 2 & 2 & 37,5 & 13,2 & 8,3 & 20,0 & 50,0 & 1 & & 85,74 & 44, & 33,3 & & 27,32 & 320,0 & & 21,4 & - \\
\hline & 59,5 & 24,1 & 62,1 & & 70,0 & - & 2 & - & 65,6 & 42,6 & 79,2 & 40,0 & 50,0 & 1 & 66,7 & 28,6 & & :75,0 & & 54,52 & 20,0 & 1 & 28,63 & 33,3 \\
\hline & 2 & 11,1 & 37,9 & - & 60,0 & 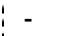 & 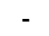 & - & 45,8 & 7,4 & 37 & 20,0 & 50,0 & 1 & 33,3 & 42,9 & - & 33,3 & 1 & 27,3 & 13,3 & + & -3 & 33,3 \\
\hline & 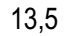 & & 10,6 & 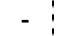 & 10,0 & 1 & 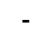 & 1 & 7,3 & 1,5 & 16,7 & 10,0 & 7,1 & - & & 42,9 & 5,6 & $: 50,0$ & 2 & 9,1 & 6,7 & + & - & - \\
\hline & 10 & 5,6 & 15,2 & - & 30,0 & 1 & - & 1 & 22,9 & 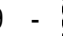 & 8,3 & 10,0 & 21,4 & 1 & 33,3 & 28,6 & - & - & - & 45,5 & - & & 14,36 & 66 \\
\hline & 5 & 3,7 & 6,1 & - & - & - & - & - & 5,2 & 2,9 & 20,8 & 10,0 & 21,4 & - & 16,7 & 71,4 & 5,6 & :58,3 & 2 & 9,1 & - & & 14,36 & 66,7 \\
\hline & 21,6 & 11,1 & 22,7 & - & 20,0 & 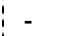 & 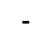 & - & 21,9 & 2,9 & 50,0 & 50,0 & 35,7 & - & 16,7 & 42,9 & - & 83,3 & 1 & 9,1 & 6,7 & & - & - \\
\hline & - & 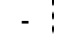 & 3,0 & - & 20,0 & - & - & - & 5,2 & 2,9 & 4,2 & & 35,7 & - & $\ldots$ & 28,6 & & 8,3 & - & 1 & 13,3 & & 7,13 & 33,3 \\
\hline & - & - & - & - & - & - & - & - & - & 5,9 & 8,3 & 20,0 & - & - & - & 14,31 & 16,7 & 25,0 & 1 & - & - & 1 & 14,3 & - \\
\hline & 8,1 & 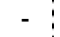 & 6,1 & - & 10,0 & 1 & - & 1 & 5,2 & - & - & - & 14,3 & - & - & - & - & - & - & 9,1 & - & & - & - \\
\hline & 5,4 & - & - & - & 10,0 & 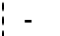 & - & 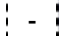 & 4,2 & 1,5 & 4,2 & 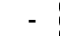 & - & - & & 14,3 & - & 41,7 & 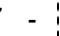 & - & - & + & - & - \\
\hline & 2,7 & - & 13,6 & - & 40,0 & 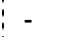 & 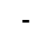 & - & 22,9 & 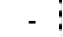 & - & - & 21,4 & 1 & 16,7 & - & - & 8,3 & - & - & - & & - & - \\
\hline & 5,4 & 1,9 & - & - & - & 1 & - & 2 & 4,2 & - & - & - & $\begin{array}{l}7,1 \\
\end{array}$ & 2 & - & 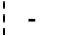 & - & - & - & - & - & & - & - \\
\hline rosat & 5,4 & & 9,1 & & & - & & & 1,0 & & 4,2 & & 7,1 & & & 14,3 & & :16,7 & & & & & 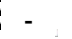 & \\
\hline \multicolumn{25}{|c|}{$\begin{array}{l}\mathrm{E}_{3}(<1,5 \mathrm{~m}): \\
\text { Taxones diferenciales de asociaciones }\end{array}$} \\
\hline & & 53,7 & 66 & 28,6 & 80,0 & 1 & 1 & 2 & & & & 30 & 57 & 2 & 16,7 & 7 & 38,9 & & 1 & 5 & 20,0 & & & \\
\hline & & 9 & 1 & & 60,0 & 1 & - & 2 & & 14, & & 20,0 & 4 & 3 & 3 & 2 & $2 ;$ & 16,7 & 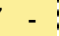 & 81,8 & 30,0 & & - & - \\
\hline & & 14 & 4 & 14,3 & 10 & 1 & - & - & 16,7 & 1,5 & 8,3 & - & 35,7 & 2 & 16,7 & 1 & 11,1 & 8,3 & - & 9,1 & 6,7 & & - & - \\
\hline & ; & 16 & 5004 & 42,9 & 50,0 & 2 & - & 2 & 6 & 4,4 & 4,2 & - & 42,9 & 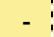 & 33,3 & 2 & 5,6 & & - & 63,6 & 13,3 & & - & - \\
\hline & 3 & 37, & 4 & 42,9 & 60,0 & 2 & - & 2 & 4 & 13,2 & & - & 57,1 & 1 & & 28,6 & 11,1 & & 1 & 45,5 & & & - & \\
\hline & 52 & 25,9 & 4 & - & 30,0 & - & - & - & 4 & 11,8 & 20,8 & - & 21,4 & - & - & 14,31 & 16,7 & 8,3 & - & 81,8 & 313,3 & & - & - \\
\hline & & - & 3 & - & 6 & 1 & - & - & 6 & 2,9 & 8,3 & - & - & - & 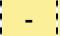 & - & 5,6 & 8,3 & - & 9,1 & 6,7 & & - & - \\
\hline & 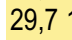 & 13,0 & 3 & - & 3 & - & - & 2 & 1 & & 4,2 & - & 35,7 & - & & 14,3 & & 8,3 & - & & & & - & - \\
\hline Erio & ? & 13,0 & 25 & - & 10 & 1 & - & 1 & 20,8 & 1,5 & 8,3 & - & 21,4 & - & 16,7 & 28,6 & 5,6 & & - & 36,4 & 6,7 & & - & - \\
\hline & 10,8 & - & 3 & - & 10,0 & 1 & - & 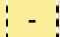 & 5,2 & - & 4,2 & - & 14,3 & - & & 28,6 & 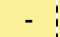 & 25,0 & - & 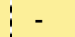 & 6,7 & & - & - \\
\hline & 5, & 1,9 & 4,5 & - & 10 & - & - & & 4,2 & & & & 7,1 & & 16,7 & 28,6 & & & & 9,1 & & & - & \\
\hline & 18,9 & 9,3 & 19,7 & & 20,0 & - & 1 & 1 & & & & 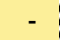 & & - & -1 & 28,6 & 11,1 & 8,3 & & & - & & 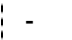 & - \\
\hline & 2,7 & 1,9 & 1,5 & - & - & - & - & - & 5,2 & 1,5 & - & - & 14,3 & - & - & - & - & - & - & 36,4 & 6,7 & & - & - \\
\hline & - & 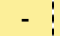 & 3 & - & 20,0 & - & & & 3,1 & - & - & & $\begin{array}{l}7,1 \\
\end{array}$ & - & - & - & - & 8,3 & - & 9,1 & 6,7 & & & 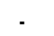 \\
\hline & 8,1 & 1,9 & 1,5 & - & & - & - & & 1,0 & & - & - & 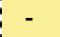 & - & & 14,3 & 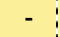 & 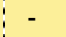 & - & 9,1 & & & & . \\
\hline & - & 1,9 & - & - & & - & 1 & - & - & - & - & 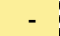 & $\begin{array}{l}7,1 \\
\end{array}$ & - & & 28,6 & - & 25,0 & 1 & 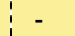 & - & & . & \\
\hline & - & & - & & 20,0 & - & & & 7,3 & 1,5 & - & - & 28,6 & 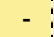 & 16,7 & & & 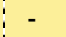 & - & 18,2 & & & 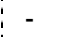 & \\
\hline & 8,1 & & 4 & - & & 1 & & & 16,7 & & & 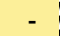 & 14,3 & & 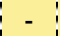 & 1 & & & - & & & & - & \\
\hline & - & 7,4 & 13 & - & 110 & - & - & - & 1 & - & - & - & i - & - & - & 14, & - & - & - & - & - & & - & \\
\hline & 5 & - & 6 , & - & 50,0 & - & - & - & 13,5 & 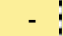 & - & - & : 7,1 & - & - & 14,3 & - & 8,3 & - & 9,1 & - & & - & - \\
\hline Varcissus triandrus & 5,4 & & 7,6 & & 20,0 & 1 & & & 17,7 & & & 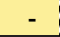 & 7,1 & & & 28,6 & & 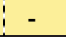 & -1 & $\pi$ & - & & - & \\
\hline
\end{tabular}


Tabla 2.- Continuación

\begin{tabular}{|c|c|c|c|c|c|c|c|c|c|c|c|c|c|c|c|c|c|c|c|c|c|c|c|c|}
\hline Columna & $1 \mathrm{a}$ & $1 \mathrm{~b}$ & $2 a$ & $2 b$ & 3 & $4 a$ & $4 b$ & 5 & $6 a$ & $6 b$ & $7 a$ & $7 b$ & $8 a$ & $8 b$ & 9 & $10 a$ & $10 \mathrm{~b}$ & $11 a$ & $11 b$ & $12 a$ & $12 b$ & 13 & $14 a$ & $14 b$ \\
\hline Peucedanum lancifolium & 24,3 & 9,3 & 16,7 & 14,3 & & - & - & & 1,0 & & & - & & - & & 14,3 & & - & - & 18,2 & & & 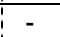 & - \\
\hline altha palustris & 27,0 & 3,7 & 33,3 & - & 40,0 & - & - & - & 19,8 & 2,9 & 12,5 & $5-$ & 7,1 & 1 & - & 14,3 & - & - & - & 9,1 & - & & - & - \\
\hline Ilium victorialis & - & 3,7 & 4,5 & - & - & - & - & - & 5,2 & - & 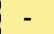 & - & 7,1 & - & - & 14,3 & 5,6 & - & - & 9,1 & & & - & - \\
\hline arex laevigata & 29,7 & 16,7 & 27,3 & - & 30,0 & - & 2 & - & 16,7 & - & - & 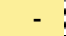 & - & 1 & & 28,6 & - & 8,3 & - & 9,1 & - & & - & - \\
\hline uercus pyrenaica & 8,1 & 1,9 & 4 & - & - & - & - & - & 2,1 & - & 4,2 & 10,0 & - & - & - & 14,3 & - & 25,0 & - & - & - & & - & - \\
\hline ardamine raphanifolia & - & - & - & - & - & - & - & - & 3,1 & 2,9 & 33,3 & 3 & : 7,1 & - & :33,3 & $3:-$ & - & - & - & - & 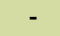 & & 14,3 & 33,3 \\
\hline astrea limbosperma & - & - & - & - & - & - & - & - & 14,6 & 8,8 & & & 7,1 & 2 & - & 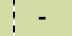 & 5,6 & 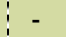 & - & 27,3 & 20,0 & & : & - \\
\hline impinella major & - & - & - & - & - & - & - & - & 2,1 & 2,9 & 16,7 & 70,0 & - & - & - & 14,3 & - & - & - & - & 6,7 & & 21,4 & - \\
\hline milax aspera & - & - & - & - & - & - & - & - & - & - & - & - & 7,1 & - & - & 14,3 & - & - & - & - & - & + & 7,1 & 33,3 \\
\hline hamaeiris foetidissima & - & - & - & - & - & - & - & - & - & - & - & - & - & - & - & 14,3 & - & 50,0 & - & - & 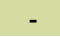 & + & - & - \\
\hline clamineus & 67,6 & 18,5 & 74,2 & 14,3 & 40,0 & - & 2 & 1 & - & - & - & - & - & - & - & - & - & - & - & - & 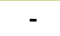 & 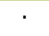 & - & - \\
\hline rianum & & 22,2 & 1,5 & 28,6 & - & - & - & - & - & - & - & - & - & - & - & - & - & - & - & - & - & . & & - \\
\hline paivae & & 3,7 & 3,0 & - & - & - & 1 & - & - & - & - & - & - & - & 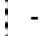 & - & - & - & - & - & - & & & - \\
\hline itanicum & 5,4 & - & - & - & - & - & - & - & - & - & - & - & - & - & - & - & - & - & - & - & - & . & - & - \\
\hline enaica & - & - & - & - & - & - & - & - & 41,7 & 41,2 & 79,2 & 60,0 & 42,9 & 2 & 100 & ) - & 16,7 & 25,0 & 1 & 36,4 & 60,0 & & - & - \\
\hline foetidus & - & - & - & - & - & - & - & 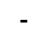 & 5,2 & 1,5 & 33,3 & 330,0 & & - & 16,7 & - & 5,6 & 16,7 & 1 & 9,1 & 6,7 & & & - \\
\hline ia alpestris & - & - & - & - & - & - & - & - & :10,4 & 8,8 & 8,3 & - & 28,6 & - & 33,3 & 3: - & - & - & - & 27,3 & 13,3 & . & - & - \\
\hline Salix & - & - & - & - & - & - & - & - & 2,1 & 2,9 & 4,2 & - & - & - & - & 14,3 & 11,1 & 16,7 & 1 & - & - & & - & - \\
\hline pismigena & - & - & - & 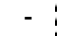 & - & - & - & - & 4,2 & 5,9 & - & - & 7,1 & 1 & . & - & - & 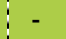 & - & 9,1 & 13,3 & & - & - \\
\hline alpina & - & - & - & - & - & - & - & - & 5,2 & - & - & - & 28,6 & - & 16,7 & - & - & 8,3 & - & 45,5 & 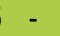 & & - & - \\
\hline npatiens & - & - & - & - & - & - & - & - & - & - & 8,3 & 20,0 & - & - & - & - & - & - & 1 & - & 6,7 & & - & - \\
\hline losa & - & 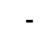 & - & - & - & - & 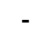 & & 11,5 & - & - & 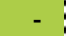 & 21,4 & 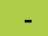 & 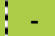 & - & - & - & - & 18,2 & & & & - \\
\hline aea & 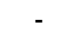 & - & - & - & - & - & 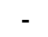 & 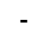 & 1,0 & - & - & - & - & - & - & - & - & 8,3 & - & - & 6,7 & & - & - \\
\hline Q.pe & - & - & - & - & - & - & - & - & - & 2,9 & - & - & 7,1 & - & - & - & - & - & - & - & 6,7 & & & - \\
\hline ckaiana & 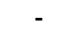 & - & - & - & - & - & - & - & 1,0 & - & - & - & 7,1 & - & - & 14,3 & - & - & - & - & - & & - & - \\
\hline naria & - & - & - & - & - & - & - & 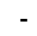 & - & - & - & 10,0 & - & - & - & - & - & - & 1 & - & - & . & - & - \\
\hline nutatus & - & - & - & - & 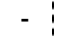 & - & - & - & 4,2 & - & - & - & - & - & - & - & - & - & - & - & - & . & - & - \\
\hline$e d \epsilon$ & - & - & - & - & - & - & - & - & - & - & - & - & - & - & - & - & - & - & - & - & & & 85,7 & 100 \\
\hline dolon & - & - & - & - & - & - & - & - & - & - & - & - & - & - & - & - & - & - & - & - & & & 35,7 & 33,3 \\
\hline yestina & - & - & - & - & - & - & - & - & - & - & - & - & - & - & - & - & - & - & - & - & 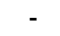 & & & 66,7 \\
\hline Salix & - & - & - & - & - & - & - & & - & - & - & - & 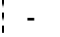 & - & - & - & - & - & - & - & & 1 & 14,3 & \\
\hline rvirens & - & - & - & - & - & - & - & - & - & - & - & - & - & - & - & - & - & - & - & - & 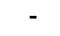 & 1 & - & 33,3 \\
\hline or & - & - & - & - & - & - & - & - & - & - & - & - & - & - & - & - & - & - & - & - & - & & : 7,1 & 33,3 \\
\hline virens & 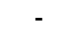 & - & - & - & - & - & - & & $3,1^{*}$ & - & - & - & :7, * & - & - & - & - & - & - & - & & & 21,4 & \\
\hline alustre & - & - & - & - & - & - & - & & - & - & - & - & - & - & 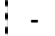 & - & - & - & - & - & 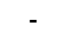 & & 14,3 & - \\
\hline $\mathrm{Cal}$ & - & - & - & - & - & - & - & & - & - & - & - & - & - & - & - & - & - & - & - & - & & 7,1 & - \\
\hline & - & - & - & - & - & - & - & & - & 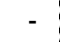 & - & - & - & - & - & - & - & - & - & - & 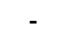 & & 7,1 & 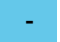 \\
\hline $\mathrm{Ca}$ & - & - & - & - & - & - & - & - & - & - & - & - & - & - & - & - & - & - & - & - & - & & 7,1 & - \\
\hline nosissim & - & - & - & - & - & - & - & - & - & - & - & - & - & - & - & - & - & - & - & - & - & & 7,1 & - \\
\hline oorei & - & - & - & - & - & - & - & & - & - & - & - & - & - & - & - & - & - & - & - & - & & 7,1 & - \\
\hline Eri & - & - & - & - & - & - & - & & - & - & - & - & - & - & & - & 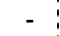 & - & - & _ & 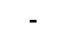 & & 7,1 & \\
\hline us & - & - & - & - & - & - & - & & - & - & - & - & - & - & - & - & - & - & - & - & - & & : 7,1 & - \\
\hline . & - & - & - & - & - & - & - & & - & - & - & - & - & - & - & 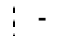 & - & - & - & - & - & & 7,1 & - \\
\hline Salix & - & - & - & - & - & - & - & & - & - & - & - & - & - & - & - & - & - & - & - & - & & 7,1 & - \\
\hline Salix eleagnos & 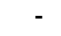 & - & - & - & - & - & - & 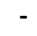 & - & - & - & 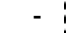 & - & - & - & - & - & - & - & - & - & & 7,1 & - \\
\hline Sambucus ebulus & 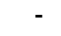 & - & - & - & - & - & - & & - & - & - & - & - & - & - & - & - & 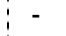 & - & 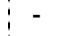 & & & 7,1 & \\
\hline Silene nemoralis & - & - & - & - & - & - & & & 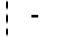 & - & - & , & - & - & & - & 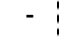 & 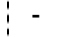 & - & & & & 7,1 & \\
\hline Viola sylvestris & - & & - & - & & - & & & - & - & - & & - & - & - & - & - & - & - & - & & & & 33,3 \\
\hline
\end{tabular}

Taxones diferenciales de subvariante de Osmunda regalis de $\mathrm{H}-\mathrm{A}$

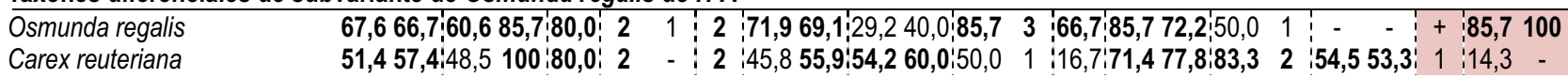

Taxones diferenciales de subasociaciones típicas (termófilas)

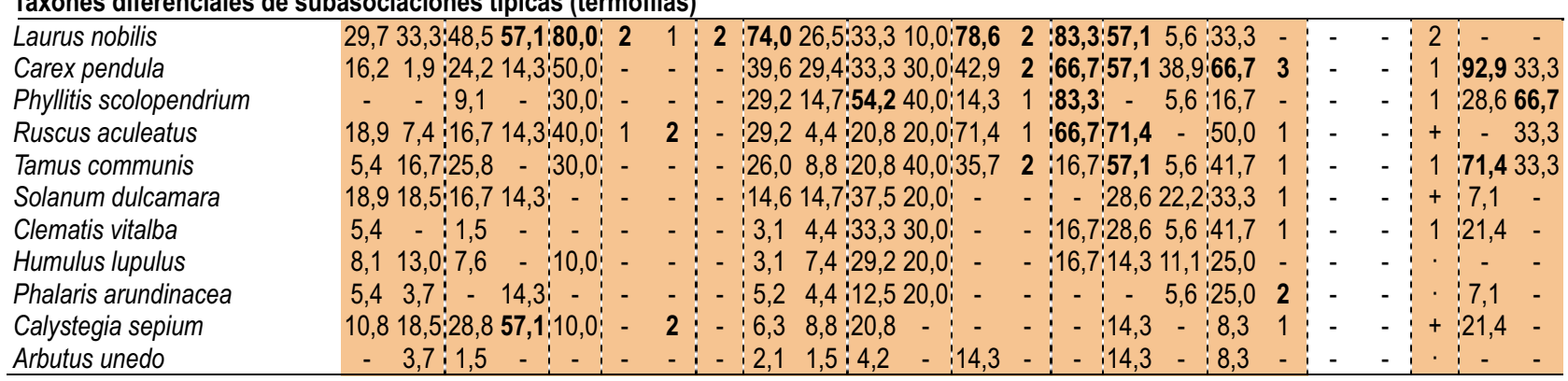


Tabla 2.- Continuación

\begin{tabular}{|c|c|c|c|c|c|c|c|c|c|c|c|c|c|c|c|c|c|c|c|c|c|c|c|c|}
\hline Columna & $1 \mathrm{a}$ & 1b & $2 a$ & $2 b$ & 3 & $4 a$ & $4 \mathrm{~b}$ & 5 & $6 a$ & $6 \mathrm{~b}$ & $7 a$ & $7 \mathrm{~b}$ & $8 a$ & $8 \mathrm{~b}$ & 9 & $10 a$ & $10 \mathrm{~b}$ & $11 \mathrm{a}$ & $11 \mathrm{~b}$ & $12 a$ & $12 b$ & 13 & $14 a$ & $14 b$ \\
\hline Asplenium onopteris & - & - & - & - & - & - & 1 & - & - & 4,4 & 4,2 & - & - & 1 & - & 14,3 & - & 8,3 & - & - & - & & - & - \\
\hline Bryonia dioica & 2,7 & 9,3 & 3,0 & - & - & - & 1 & - & - & - & - & - & - & - & 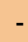 & 14,3 & 5,6 & - & - & - & - & & - & - \\
\hline Saponaria officinalis & 5,4 & 3,7 & 3,0 & & 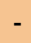 & 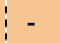 & . & & 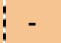 & 1,5 & - & - & - & - & & $e^{-}$ & - & 33,3 & - & - & - & & 14,3 & - \\
\hline Rubia peregrina & - & - & - & - & - & - & - & - & 1,0 & - & - & - & - & 2 & - & 28,6 & - & - & - & - & - & + & - & - \\
\hline Osyris alba & - & 3,7 & - & - & - & - & - & - & - & - & - & - & - & - & - & - & - & - & - & - & - & & - & - \\
\hline Daphne gnidium & - & 3,7 & 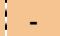 & - & & - & - & & - & - & - & - & - & - & 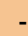 & - & - & - & - & - & - & & - & - \\
\hline Arisarum vulgare & - & 1,9 & - & - & - & - & - & - & - & - & - & - & - & - & - & - & - & - & - & - & _ & & - & - \\
\hline
\end{tabular}

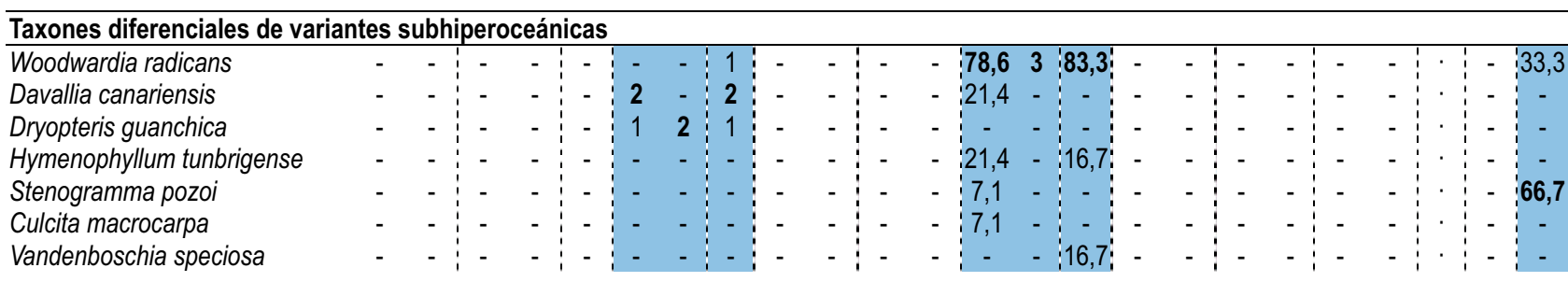

Taxones diferenciales de variantes semihiperoceánicas en V-A

\begin{tabular}{|c|c|c|c|c|c|c|c|c|c|c|c|c|c|c|c|c|c|c|c|}
\hline Fraxinus angustifolia & $48,668,5 ; 7,9$ & $1,4: 50,0$ & 2 & 1 & 2 & - & - & 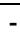 & - & & 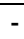 & & $85,777,8: 58,3$ & 3 & - & - & . & - & - \\
\hline Ulmus minor & $-1,5$ & - & - & 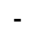 & - & - & - & - & & - & - & & $14,311,1,58,3$ & 1 & - & - & . & - & - \\
\hline Fr. excelsior x Fr. angustifolia & $13,5 \quad 1,9: 18,2$ & - & - & 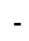 & - & - & 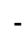 & - & - & 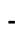 & & & $-42,9-41,7$ & - & - & - & & - & \\
\hline Salix salviifolia & $2,7 \quad 1,9$ & - & - & - & - & - & & - & 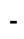 & 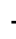 & & & - $14,327,8: 16,7$ & - & 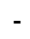 & - & . & - & \\
\hline Anemone albida & $\begin{array}{ll:l}5,4 & 1,9 & 4,5\end{array}$ & - & - & - & - & - & - & - & - & 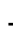 & & - & $-11,1-$ & - & - & - & . & - & - \\
\hline Salix atrocinerea x S. salviifolia & $2,7 \quad 9,3 \quad-$ & 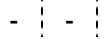 & - & & - & - & & . & 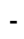 & 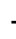 & & - & $-8,3$ & - & - & & . & - & \\
\hline
\end{tabular}

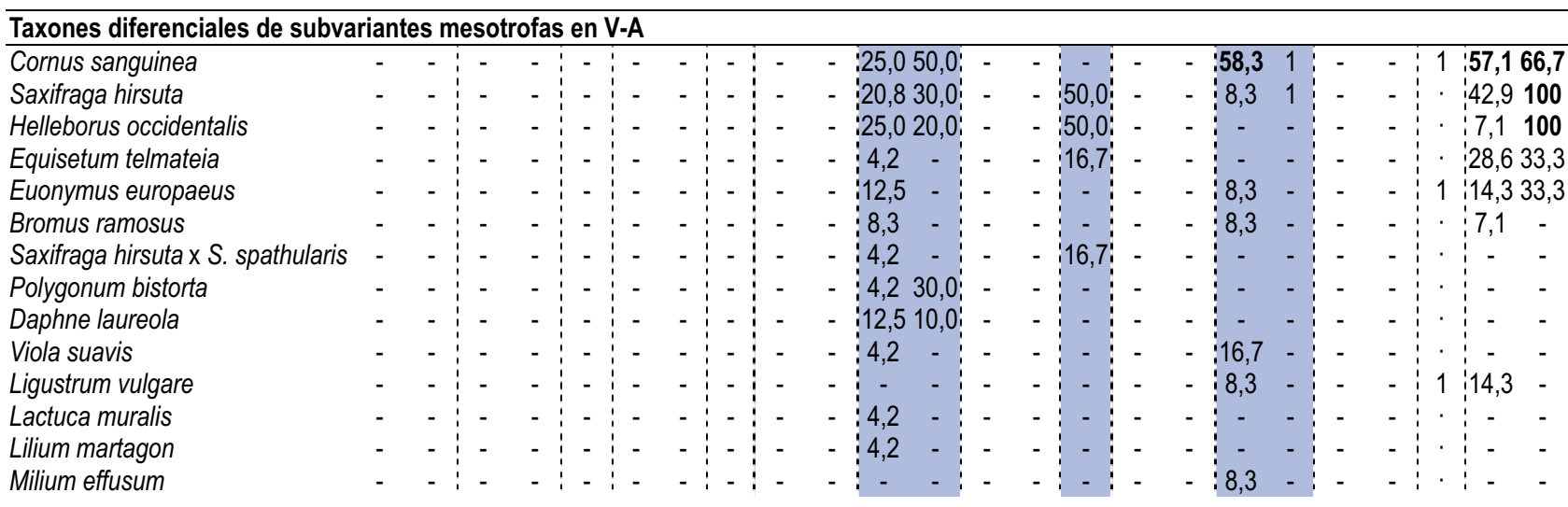

Taxones diferenciales de subvariantes mesotrofas en S-A

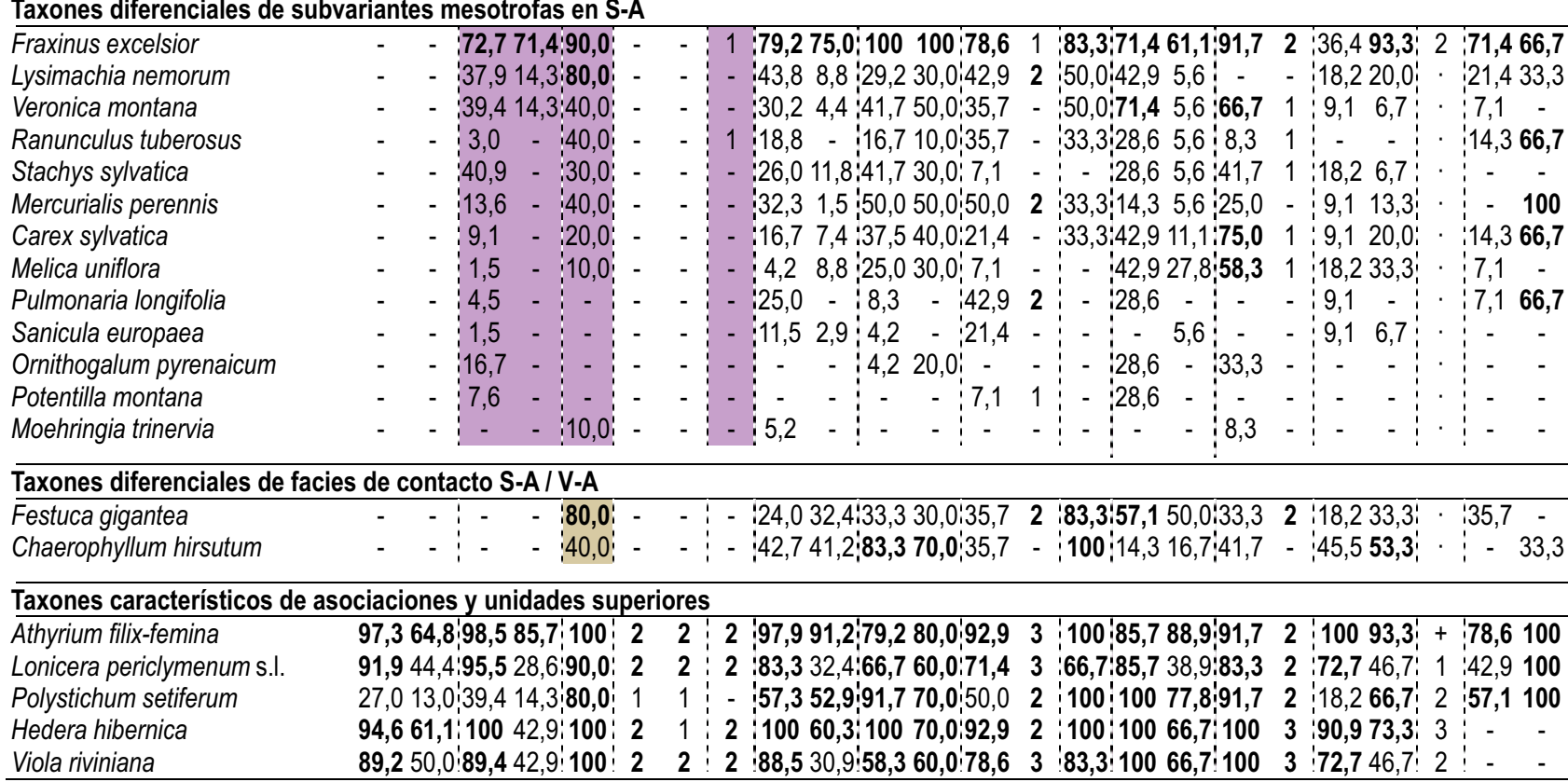


Tabla 2.- Continuación

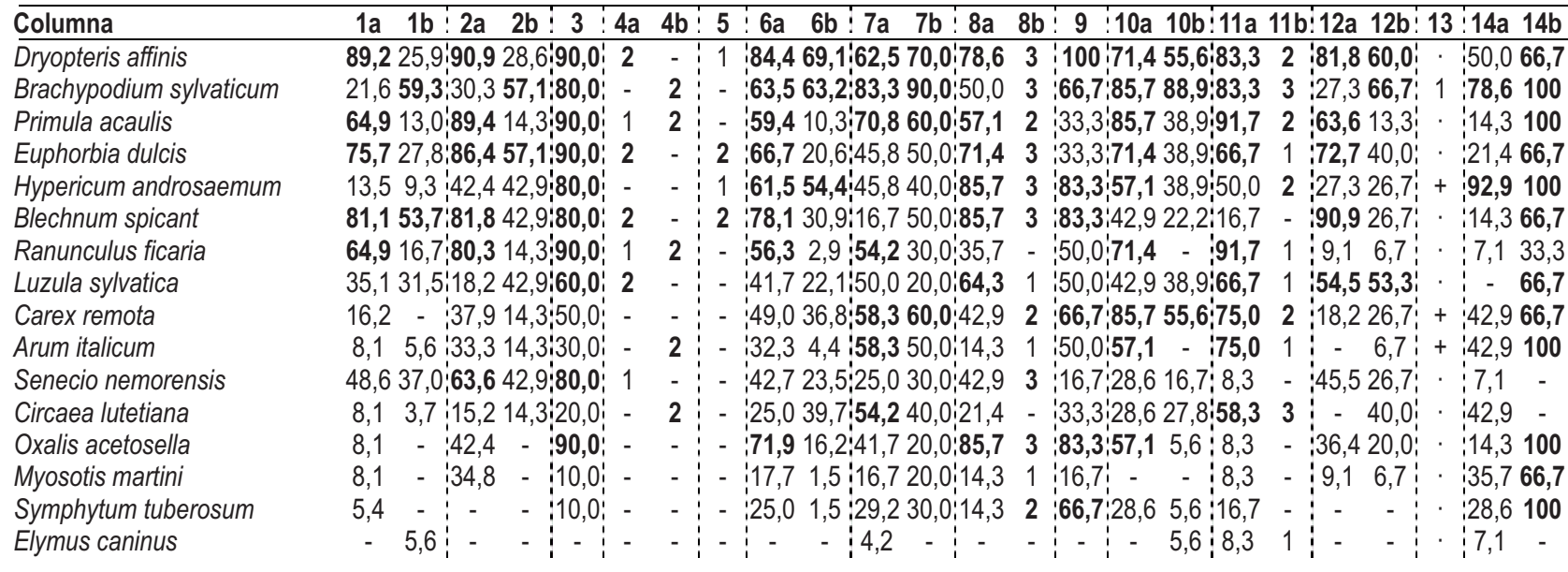

\section{Taxones característicos de Quercetea robori-petraeae y Carpino-Fagetea sylvaticae}

\begin{tabular}{|c|c|c|c|c|c|c|}
\hline Euphorbia amygdaloides & $27,033,3,48,542,9 ; 70,0$ & 1 & $\begin{array}{l:l:}1 & 53,126,5 \\
\end{array}$ & 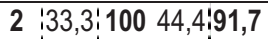 & $2 \mid 27,326,7 !$ & $+57,133,3$ \\
\hline Ajuga reptans & $35,114,8,71,271,4,100$ & 2 & - $\quad 68,8 \quad 8,8,54,240,0 ; 71,4$ & 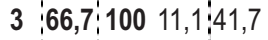 & $1: 18,213,3$ & $21,466,7$ \\
\hline Crepis lampsanoides & $43,224,130,3-70,0$ & 1 & - $34,423,5,45,830,0,57,1$ & $33,3,57,138,9,50,0$ & $1: 27,340,0$ & 66,7 \\
\hline Teucrium scorodonia & $83,844,4,66,714,3) 70,0$ & 2 & 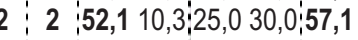 & $57,122,2,50,0$ & $2 \nmid 90,96,7\}$ & 7,1 \\
\hline Holcus mollis & $78,49,3) 84,8 \quad 14,3,80,0$ & 1 & $\begin{array}{l:ll:l}1 & 72,9 & 5,9 & 29,2 \\
30,0 & 78,6\end{array}$ & 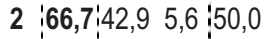 & - $81,826,7$ & 7,1 \\
\hline Dryopteris dilatata & $-60,6-90,0$ & 1 & 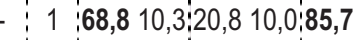 & $\begin{array}{l:ll:l}100 & 42,9 & 5,6 & 8,3\end{array}$ & $63,613,3$ & $7,1 \quad 100$ \\
\hline Stellaria holostea & 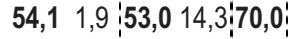 & - & - $32,3 \quad 2,9: 41,720,0: 14,3$ & $1: 33,3,42,9$ & $1 \quad 54,513,3$ & 66,7 \\
\hline Aquilegia vulgaris & $2,7 \quad 3,7: 18,2 \quad-$ & - & - $35,45,9,16,720,0,28,6$ & $\begin{array}{l:l:l} & 16,7 & 42,9 \\
22,2,33,3\end{array}$ & $18,220,0$ & 100 \\
\hline Anemone nemorosa & $21,6-28,8$ & 2 & $\begin{array}{l:ll:l} & 43,8 & 7,4 & 20,8 \\
20,0 & 57,1\end{array}$ & $33,3: 28,611,1: 41,7$ & 27,3 & 66,7 \\
\hline Potentilla sterilis & $24,3-42,4$ & - & - $26,0 \quad 1,5: 37,520,0: 14,3$ & $50,0: 42,9-66,7$ & 6,7 & $1,433,3$ \\
\hline Hyacinthoides non-scripta & $\begin{array}{ll:l}24,3 & 3,7 & 45,5\end{array}$ & 1 & - $\begin{array}{ll:lll}40,6 & 2,9 & 12,5 & 30,0 & 14,3\end{array}$ & $\begin{array}{l:l}-66,7 \\
\end{array}$ & 1 & 66,7 \\
\hline Polypodium vulgare & $75,7-68,2-90,0$ & 1 & 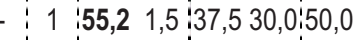 & $16,7: 57,1$ & - 45,5 & . \\
\hline Stachys officinalis & $5,4 \quad 3,7: 16,7 \quad 14,3: 40,0)$ & - & - 19,8 5,9 & - $28,65,6$ & 9,1 & $-\quad 33,3$ \\
\hline Dryopteris filix-mas & $-6,1$ & - & $\begin{array}{l:llll}-12,5 & - & 45,8 & 10,0 & 7,1\end{array}$ & $\begin{array}{l}-42,9-66,7 \\
\end{array}$ & - $27,36,7$ & - 7,1 \\
\hline Poa nemoralis & $2,7 \quad 16,7:-$ & 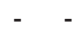 & $\begin{array}{l:lll} & 7,3 & 27,9,25,0 & -\end{array}$ & - $\quad 44,4,16,7$ & $-53,3$ & - $: 7,1$ \\
\hline
\end{tabular}

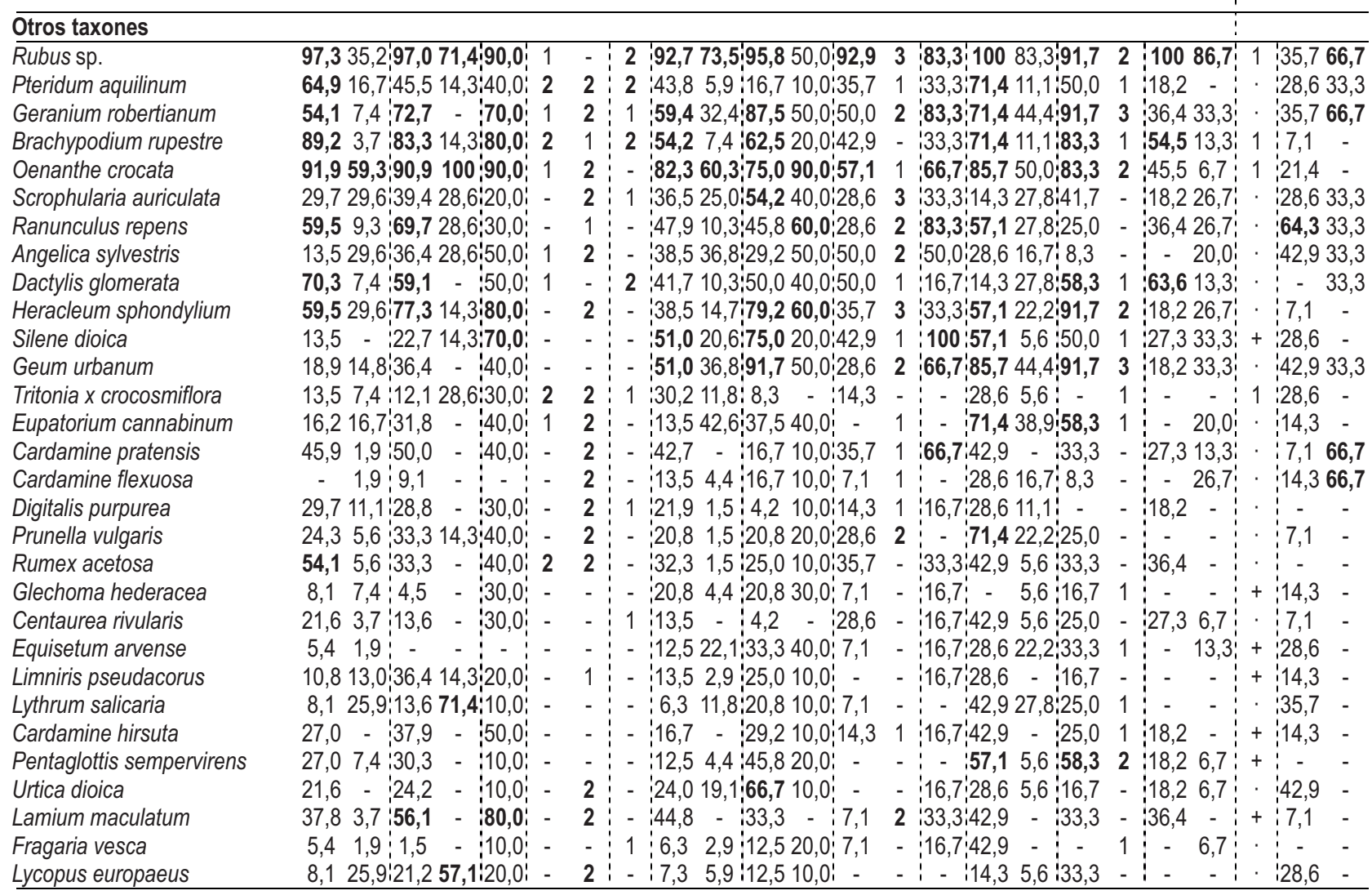


Tabla 2.- Continuación

\begin{tabular}{|c|c|c|c|c|c|c|c|c|c|c|c|c|c|c|c|c|c|c|c|c|c|c|c|c|}
\hline olumna & $1 \mathrm{a}$ & 1b ! & $2 a$ & $2 \mathrm{~b}$ & 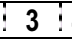 & $4 a$ & $4 b$ & 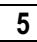 & 6a & 6b! & $7 a$ & /D ! & $8 \mathrm{a}$ & 8D & 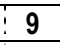 & !10a & 10b: & !11a & 110 & $12 a$ & $12 b$ : & $13 !$ & !14a 1 & 14b \\
\hline hrysosplenium oppositifolium & 5,4 & 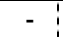 & 10,6 & - & $|30,0|$ & 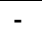 & & & 28,1 & 1,5 & 45,8 & 10,0 & 21,4 & & 66,7 & & & 8,3 & - & 27,3 & 20,0 & & -3 & 33,3 \\
\hline lipendula ulmaria & 5,4 & 13,0 & 24,2 & 42,9 & 20,0 & - & 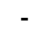 & & 9,4 & 8,8 & :41,7 & 4( & & & & 14,3 & 16,7 & 66,7 & 1 & - & 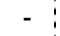 & & 50,0 & - \\
\hline rupestris & 5 & - & 37,9 & - & $40,0\}$ & 1 & & & 32,3 & -1 & 29,22 & 20,0 & 21,4 & & 50,0 & 57,1 & 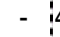 & 1,7 & 1 & 18,2 & & & & - \\
\hline & 5 & 7,4 & 7,6 & 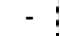 & 10,0 & 1 & 1 & & 4,2 & & 4,2 & & & 2 & & & - & - & - & 9,1 & - & & 14,33 & 33,3 \\
\hline des & 5,4 & $9,3:$ & 6,1 & - & 10,0 & 1 & & & 10,4 & 5,9 & 4,2 & - & 14,3 & 2 & 16,7 & 42,9 & - & - & - & $\begin{array}{l}9,1 \\
9\end{array}$ & - & & - & - \\
\hline his & 21,6 & 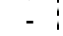 & 7,6 & - & 10,0 & - & - & - & $\begin{array}{l}19,8 \\
\end{array}$ & 1,5 & 33,3 & 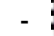 & 7,1 & - & 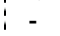 & 42,9 & 5,6 & 50,0 & 1 & 18,2 & - & & 7,1 & - \\
\hline Ilarıs & 24,32 & 20,4 & 18,2 & $28,6^{\prime}$ & & - & - & 1 & 15,6 & 2,9 & 12,5 & & 7,1 & 1 & & 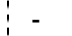 & - & :16,7 & - & 54,5 & & & 21,4 & - \\
\hline rine & 24,3 & 1,9 & 30,3 & & 10,0 & - & 2 & & 17,7 & 2,9 & 25,0 & 10,0 & & - & & 28,6 & 11,1 & 50,0 & 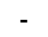 & - & - & & 14,3 & - \\
\hline atica & 2,7 & 1,9 & 13,6 & 14,3 & & - & & 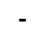 & 8,3 & 7,4 & 8 & & 14,3 & 1 & 16,7 & & - & - & - & - & - & + & 42,9 & - \\
\hline Im & 3 & 1,9 & 6,1 & 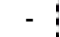 & 30,0 & - & - & - & 13,5 & 1,5 & 12,5 & 10,0 & & 1 & 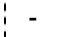 & 14,3 & & 8,3 & - & 9 & 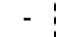 & . & - & - \\
\hline iar & 5 & & $\begin{array}{l}1,5 \\
\end{array}$ & - & & - & - & - & 4,2 & & $54,2\}$ & 30,0 & - & - & - & 57,1 & 22,2 & 75,0 & 2 & - & 6,7 & + & 21,4 & - \\
\hline lorum & 16,2 & 1,9 & $\begin{array}{l}9,1 \\
9\end{array}$ & 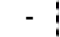 & $10,0\}$ & 1 & 1 & & 11,5 & 13,2 & 29,2 & 10,0 & & - & - & & -1 & 16,7 & - & - & - & . & $\begin{array}{l}7,1 \\
\end{array}$ & - \\
\hline officinale & 8 & - & 9,1 & - & & - & - & - & 7,7 & 1,5 & 41,72 & 20,0 & - & - & - & 28,6 & $-:$ & :41,7 & 1 & - & - & $r$ & 7,13 & 33,3 \\
\hline era & 2 , & $1,9:$ & $\begin{array}{l}9,1 \\
\end{array}$ & - & & - & - & - & 1 & - & 8,3 & 10,0 & $\begin{array}{l}7,1 \\
\end{array}$ & 1 & - & 14,3 & - & - & 1 & $\begin{array}{l}9,1 \\
\end{array}$ & - & 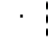 & - & - \\
\hline$a$ & & 11,1 & 3,0 & 28,6 & & 1 & - & 2 & 2 & & & & 7,1 & & & 28,6 & 11,1 & - & - & 9,1 & & & - & - \\
\hline ustre & & 3,7 & 12,1 & + & $30,0\}$ & - & - & & 2,1 & - & 8,3 & 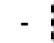 & (,1 & - & - & - & 5,6 & 8,3 & - & 9,1 & 6,7 & . & - & - \\
\hline m perss & & 1,9 & 13 & 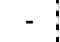 & $1-1$ & - & - & & 8,3 & - & 4,2 & - & l,1 & - & 16,7 & 14,3 & 5,6 & 8,3 & - & 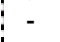 & - & & 21,4 & - \\
\hline roni & & - & 13 & - & 40,0 & - & - & & 15,6 & 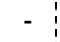 & 37,5 & - & 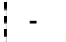 & - & 16,7 & 42,9 & 1 & :50,0 & 1 & $\begin{array}{l}18,2 \\
\end{array}$ & 6,7 & & - & $\begin{array}{lll}- & -1\end{array}$ \\
\hline eolens & 5 & 1,9 & 1,5 & 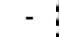 & - & - & 1 & & 1 & 5,9 & 4,2 & & - & - & & 14,3 & 5,6 & - & - & - & - & & 14,3 & - \\
\hline Cami & 2 & - & 6,1 & - & 10,0 & 1 & & & 6,3 & . & 8,32 & 20,0 & - & 1 & 33,3 & & - & 8,3 & - & - & - & & & - \\
\hline 1100 & & 1,9 & 7,6 & - & & - & - & - & 11,5 & - & 4,2 & - & - & 1 & 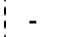 & 28,6 & 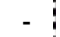 & 8,3 & - & 18,2 & - & + & - & - \\
\hline ifolius & 5 & 1,9 & 12,1 & - & 10,0 & - & 1 & - & 3 & - & 8,3 & - & - & - & - & - & - & 25,0 & - & 9 & - & . & 7,1 & - \\
\hline 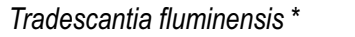 & 2 , & 1,9 & 1,5 & 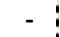 & 10,0 & 1 & 1 & 1 & 1,3 & & - & - & - & - & - & 14,3 & -1 & 1 - & - & - & 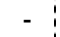 & & - & - \\
\hline veright & 8, & 3,7 & $\begin{array}{l}1,5 \\
\end{array}$ & 14,3 & & - & - & - & - & 1,5 & - & - & - & - & - & 14,3 & 11,1 & - & 2 & - & 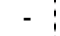 & & 21,4 & - \\
\hline & - & -1 & $\begin{array}{l}3,0 \\
\end{array}$ & - & & - & - & - & 8,3 & 1,5 & $29,2\}$ & 30,0 & - & - & - & - & - & 41,7 & - & 9 & 6,7 & . & $\begin{array}{l}7,1 \\
\end{array}$ & - \\
\hline ticus & 24,3 & 5,6 & 25,8 & - & & 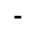 & - & 1 & 4,2 & 2,9 & & & & 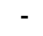 & & 14,3 & 5,6 & & - & & & & 35,7 & - \\
\hline er & 2 & 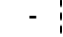 & $\begin{array}{l}1, \\
.\end{array}$ & - & - & - & 1 & - & 1,0 & 1,5 & 16,7 & - & - & - & - & 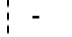 & 5,6 & $\begin{array}{l}8,3 \\
\end{array}$ & - & - & - & & - & - \\
\hline Dlys & 8,1 & - & 1, & - & 10,0 & - & - & & - & - & 4,2 & 10,0 & - & - & 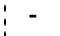 & 14,3 & - & 25,0 & 1 & - & 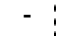 & & - & - \\
\hline (2) & - & 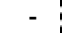 & 1 & - & & - & - & 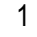 & 2,1 & - & 20,8 & 10,0 & - & - & $\begin{array}{l}33,3 \\
\end{array}$ & $3:-$ & 5,6 & 16,7 & - & - & 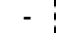 & & - & - \\
\hline & & - & 1 & - & & - & - & - & 0 & & :12,5 & 10,0 & - & - & 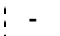 & - & - & 25,0 & 1 & - & & & :28,6 & - \\
\hline & & - & 4 & 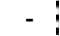 & & - & - & & 0 & 1,5 & :16,7 & - & - & - & & 14,3 & - & :16,7 & 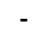 & 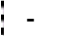 & & & $\begin{array}{l}7,1 \\
\end{array}$ & - \\
\hline IIm & 8, & 9,3 & 3 & - & $10,0\}$ & - & - & & 4, & 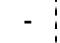 & 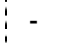 & - & 7,1 & - & - & 14,3 & - & - & - & - & 6,7 & & - & $\begin{array}{l}- \\
-\end{array}$ \\
\hline$n=$ & 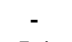 & 3,7 & 6 & 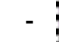 & 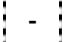 & - & 2 & & 6,3 & - & 4,2 & 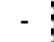 & 7,1 & 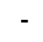 & - & 14,3 & - & 8,3 & - & - & & & - & $\begin{array}{ll}- \\
-\end{array}$ \\
\hline & 5 & 3,7 & 4 & - & & - & & & 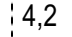 & 7,4 & 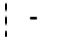 & - & - & - & & 14,3 & 5,6 & - & - & 9,1 & & & - & - \\
\hline Iboco & & - & 12,1 & - & 20,0 & 1 & - & & 1,0 & - & - & 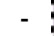 & 7,1 & - & - & 14,3 & - & - & - & - & & & - & - \\
\hline ecta & & 18,5 & 9 & - & & - & - & & 5 , & - & - & - & 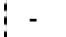 & 1 & - & 14,3 & - & - & - & 9 & & & - & - \\
\hline istre & & 9,3 & 13 & - & & 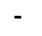 & & & 3,1 & & 4,2 & & - & 2 & & & - & 16,7 & - & - & & & 14,3 & $\begin{array}{ll}- \\
-\end{array}$ \\
\hline um am & & 3,7 & 7 & - & 10,0 & - & - & & 16,7 & & & - & 7,1 & - & & 14,3 & - & - & - & 18,2 & & & & \\
\hline iflor & 2 , & $1,9:$ & 1, & - & & - & - & & 1,0 & & & 10,0 & - & - & - & 14,3 & & 8,3 & - & - & & & & \\
\hline & & 1,9 & 1, & - & & & - & & 1,0 & 1,5 & 4,2 & - & & - & & & - & - & - & & & & :14,3 & - \\
\hline & & 1,9 & - & - & & - & - & & 1,0 & 5,9 & & & - & 1 & & & 5,6 & 8,3 & - & & & & - & - \\
\hline & & - & 3, & - & & & 1 & & - & & 8,3 & 10,0 & - & - & - & & - & :16,7 & - & $\begin{array}{ll}- \\
-\end{array}$ & & & 7,1 & - \\
\hline , a & & - & 1, & - & & 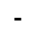 & - & - & 2,1 & & 12,5 & 10,0 & 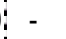 & - & 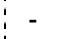 & 14,3 & - & : 8,3 & - & - & & & - & - \\
\hline & & 1,9 & 1,5 & - & & - & 1 & - & 4,2 & & 4,2 & 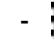 & - & - & 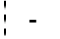 & 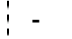 & - & 25,0 & - & - & & & & - \\
\hline rapterum & & - & 4,5 & 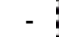 & & 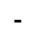 & & & 4,2 & 1,5 & & & - & - & 16,7 & 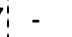 & & - & - & 18,2 & & & - & 33,3 \\
\hline & & 3,7 & :10,6 & - & & & & & L, & 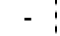 & 4,2 & & - & 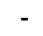 & - & - & - & - & - & 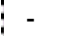 & & & 7,1 & - \\
\hline ens & & - & - & - & & & & & 1,0 & & 4,2 & & - & - & & 14,3 & - & 8,3 & - & - & 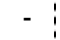 & + & 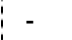 & - \\
\hline & $2, I$ & $\begin{array}{l}- \\
\end{array}$ & 1, & - & & & & & 2,1 & 1,5 & - & 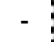 & - & - & - & - & 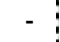 & 8,3 & 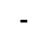 & 9,1 & 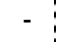 & & - & - \\
\hline & 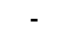 & 1,9 & 1 , & & & & & & 1 & & & & 7,1 & 1 & & & 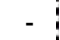 & - & - & - & & & 7,1 & \\
\hline & 2,7 & - & $\begin{array}{l}7,6 \\
\end{array}$ & - & 20,0 & - & & & 12,5 & - & & & 14,3 & - & - & - & - & 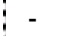 & - & 9,1 & - & & - & - \\
\hline & -3 & 37,0 & 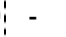 & 14,3 & 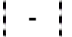 & - & 2 & - & - & - & - & - & - & 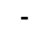 & - & - & 5,6 & - & - & - & - & & 28,63 & 3,3 \\
\hline die & 8,1 & 5, & 27,3 & - & 30,0 & - & & - & 10,4 & & - & & - & & - & - & & - & - & & & & 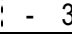 & 33,4 \\
\hline
\end{tabular}

Procedencia de los inventarios:

Col. 1a: columna sintética a partir de las tablas la y lb (ANEXO).

Col. 1b: columna sintética apartir de: Amigo et al. (1987): tabla 1: invs. 2-17 y 21; Bellot (1968): Cuadro 6: invs. 1-4, 8-10, 12-14,

16 y 21; Casaseca (1959): Tabla pag. 346 bis: 6 invs.; Dalda (1972): Cuadro no 10: invs. 4 y 5.; Honrado et al. (2002): Tabla

1: invs. 1,2 y 4-6; Izco et al. (1994): Tabla 6: invs. 1 y 2 y Pulgar (1999): Tabla 5: 9 invs.

Col. 2a: columna sintética a partir de las tablas Ila, Ilb y llc (ANEXO).

Col. 2b: columna sintética a partir de: Amigo et al. (1987): tabla 1: invs. 1, 18-20, 23 y 25; Honrado et al. (2002): Tabla 1: inv. 3.

Col. 3: columna sintética a partir de los invs. 1-10 de la tabla III (ANEXO).

Col. 4a: columna sintética a partir de los invs. 11 y 12 de la tabla III (ANEXO).

Col. 4b: columna sintética a partir de Honrado et al. (2002): Tabla 1: inv. 7 y 8. 
Col. 5: columna sintética a partir los invs. 13 y 14 de la tabla III (ANEXO).

Col. 6a: columna sintética a partir de las tablas IVa, IVb, IVc y IVd (ANEXO).

Col. 6b: columna sintética a partir de Amigo et al. (1987): tabla 3: invs. 1, 6-9, 12-23, 25-27, 29, 30 y 33; López Castro et al. (2015): Tabla 3: inv. 6; Mayor \& Fernández (2007): Tabla 4: invs. 1-5, 7-14, 17, $19-31$ y 33-35; Rodríguez Guitián et al. (2014): Tabla 10: invs. 17 y 18; Romero Buján (1993): Tabla 5: invs. 4-7, 10 y 11; Silva-Pando (1990): Tabla 17: invs. 1, 2 y 4-7.

Col. 7a: columna sintética a partir de la tabla $\mathrm{V}$ (ANEXO)

Col. 7b: columna sintética a partir de Amigo et al. (1987): tabla 3: inv. 28; Mayor \& Fernández (2007): Tabla 4: invs. 15, 16 y 32; Rodríguez Guitián et al. (2014): Tabla 10: invs. 20-23; Romero Buján (1993): Tabla 5: invs. 1 y 8.

Col. 8a: columna sintética a partir de la tabla VI (ANEXO).

Col. 8b: columna sintética a partir de Amigo et al. (1987): tabla 3: inv. 24; López Castro et al. (2015): Tabla 3: inv. 4 y 5.

Col. 9: columna sintética a partir de los invs. 1-6 de la tabla VII (ANEXO).

Col. 10a: columna sintética a partir de los invs. 7-13 de la tabla VII (ANEXO).

Col. 10b: columna sintética a partir de Amigo et al. (1987): tabla 1: inv. 22, 32 y 34-37; Bellot (1968): Cuadro 16: inv. 17; Mayor \& Fernández (2007): Tabla 4: inv. 6; Romero Buján (1993): Tabla 5: inv. 2 y 9; Tabla 6: invs. 2-7; Silva-Pando (1990): Tabla 17: invs. 15 y 17.

Col. 11a: columna sintética a partir de los invs. 14-25 de la tabla VII (ANEXO).

Col. 11b: columna sintética a partir de Rodríguez Guitián et al. (2014): Tabla 10: inv. 24.; Romero Buján (1993): Tabla 6: invs. 1 y 8.

Col. 12a: columna sintética a partir de los invs. 1-11 de la tabla VIII (ANEXO).

Col. 12b: columna sintética a partir de Amigo et al. (1987): tabla 3: invs. 2-5, 10 y 11; Mayor \& Fernández (2007): Tabla 4: inv. 18; Rodríguez Guitián et al. (2014): Tabla 10: inv. 19; Romero Buján (1993): Tabla 5: inv. 3; Silva-Pando (1990): Tabla 17: invs. 3, 8,9 y $12-14$.

Col. 13: inv. 12, tabla VIII (ANEXO).

Col. 14a: columna sintética a partir de Amigo et al. (1987): tabla 2: invs. 14 y 17; Biurrun et al. (1994): tabla 1: invs. 1, 4-7 y 9; Braun-Blanquet (1967): Tabla 28: inv. 9; Catalán (1987): tabla 5: invs. 1, 2, 4 y 5; García-Mijangos et al. (2004): Tabla 14: inv. 1; Onaindía et al. (1987): Tabla 1: inv. 2.

Col. 14b: columna sintética a partir de Amigo et al. (1987): tabla 2: invs. 15 y 16; Biurrun et al. (1994): tabla 1: inv. 3.

Por otra parte, si el muestreo se realiza en una unidad de tamaño suficiente pero sin tener en cuenta la fenología de las especies presentes, incluirá solamente una parte, en ocasiones no suficientemente significativa a efectos fitosociológicos, de las plantas que integran dicha comunidad $y$, nuevamente, su descripción a partir de un listado parcial de taxones puede dar lugar a problemas interpretativos a posteriori. Esta cuestión podría ser la causa de la ausencia o presencia testimonial de un grupo bastante numeroso de especies de fenología otoñal, invernal y pre-estival, entre las que se encuentran diversos geófitos, como azafranes silvestres (Crocus spp.), narcisos (Narcissus spp.), jacintos (Hyacinthoides spp.) o algunos ranúnculos (Ranunculus ficaria, $R$. tuberosus), en la mayor parte de los inventarios contenidos en las referencias bibliográficas consultadas para este trabajo. Análogamente, también se echa en falta una mayor presencia de taxones cuyo desarrollo de sus estructuras aéreas tiene lugar bien avanzado el verano, como ocurre con diversas trepadoras (Bryonia dioica, Calystegia sepium, Humulus lupulus, Solanum dulcamara). Por su particular fenología, estas especies son difíciles (o en algunos casos imposible) de detectar a finales de primavera o inicios de verano, período en el que se han concentrado tradicionalmente los trabajos de campo relacionados con el estudio de la vegetación leñosa en nuestras latitudes, particularmente aquella que se desarrolla en medios húmedos.

Los aspectos comentados tienen trascendencia a la hora de comparar la información contenida en las referencias previas sobre los bosques de ribera dentro del ámbito de estudio con la obtenida en nuestros trabajos de campo. Para facilitar la comprensión del alcance de estos fenómenos se han construido las gráficas de la Figura 3.
En la primera de ellas (Figura 3a) se comparan los valores mínimo, medio y máximo del número de especies registrado en los inventarios publicados consultados para la realización de este trabajo y los de elaboración propia. En conjunto, se constata una gran variación en dichos valores, oscilando los valores mínimo y máximo por inventario entre 7 y 111 taxones. Esta variación tan abultada en la composición florística de los inventarios no sólo se observa al comparar las diferentes asociaciones comentadas, sino que se presentan en los datos aportados por diferentes autores para una misma comunidad. La comparación de la distribución de estos mismos valores por medio de los intervalos mostrados en la Figura $3 \mathrm{~b}$ muestra que sus modas se encuentran bastante distanciadas: en los primeros, la clase más representada $(36,0 \%$ de las muestras) corresponde con inventarios de entre 21 y 30 taxones de plantas vasculares, mientras que en los segundos, las clases $41-50$ y $51-60$ se reparten, prácticamente a partes iguales, el $61,4 \%$ de las muestras.

La heterogeneidad encontrada en lo relativo al número de especies registradas en los dos conjuntos de inventarios estudiados se observa, igualmente, al estudiar el tamaño de las unidades de muestreo empleadas (Figura 3c): el 65,1\% de los inventarios procedentes de las referencias consultadas se han realizado en áreas de muestreo con tamaño igual o inferior a $150 \mathrm{~m}^{2}$, destacando, por extremadamente pequeñas $\left(10-100 \mathrm{~m}^{2}\right)$, las superficies utilizadas por algunos autores, como Dalda (1972), Romero (1993) o Mayor \& Fernández (2007). En algunos casos (p.ej.: Bellot 1968, Honrado et al. 2002), este tipo de análisis no se ha podido realizar, al no incluir las tablas de inventarios la información acerca del área de muestreo, cuestión que dificulta la comparación de información en estudios relacionados con el análisis de la biodiversidad. 

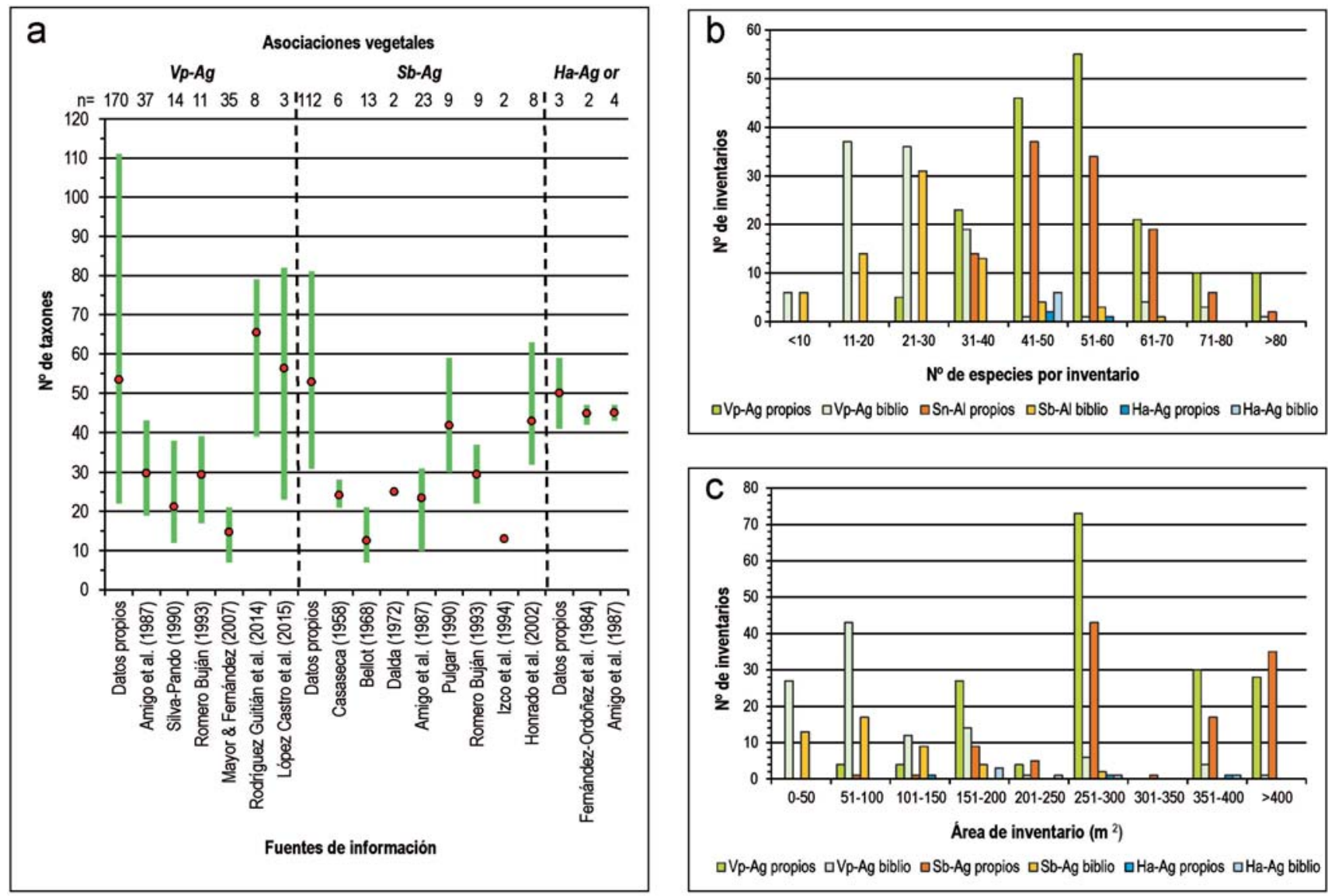

$\square$ Vp-Ag propios $\square$ Vp-Ag biblio $\square$ Sn-Al propios $\square$ Sb-Al biblio $\square$ Ha-Ag propios $\square \mathrm{Ha}-\mathrm{Ag}$ biblio

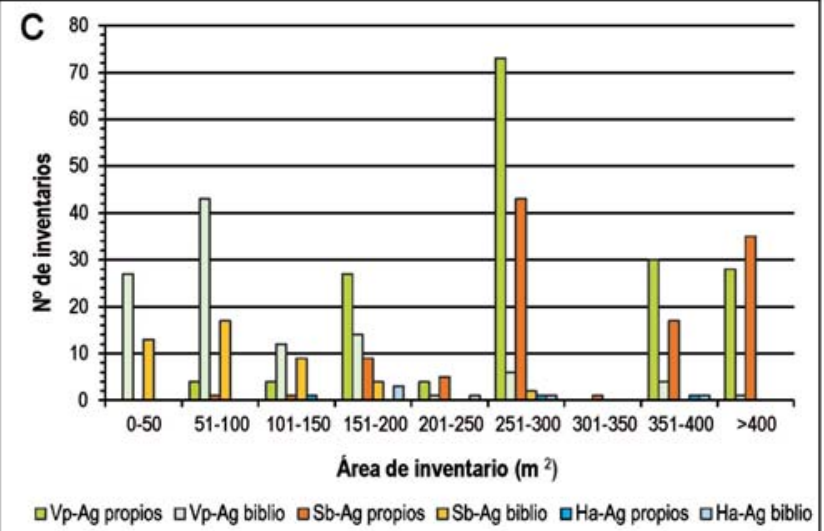

Figura 3.- a: comparación de los valores mínimo, medio (círculo rojo) y máximo de especies de plantas vasculares presentes en distintas tablas de inventarios tomadas de las fuentes bibliográficas utilizadas y los de elaboración propia. b: comparación de las distribuciones de la riqueza en taxones vegetales de inventarios tomados de las fuentes bibliográficas utilizadas y los de elaboración propia. c: comparación de las distribuciones de las superficies de muestreo de inventarios tomados de las fuentes bibliográficas utilizadas y los de elaboración propia. Acrónimos: Vp-Ag: Valeriano pyrenaicae-Alnetum glutinosae; Sb-Ag: Senecioni bayonnensis-Alnetum glutinosae; Ha-Agor: Hyperico androsaemi-Alnetum glutinosae subas. osmundetosum regalidis

La utilización de unidades de muestreo no suficientemente amplias para caracterizar ciertos tipos de vegetación ha sido detectada en muchos otros territorios europeos y constituye una fuente de problemas en el análisis e interpretación de los patrones de variación de tipos de vegetación de amplia distribución (Dengler et al. 2009, Biurrun et al. 2016). A este respecto, diferentes autores han discutido acerca del área mínima de inventario que se debería utilizar en la caracterización florística de las comunidades arboladas de las áreas templadas de Europa, existiendo un consenso generalizado acerca de que ésta debería ser igual o superior a $300 \mathrm{~m}^{2}$ (Braun-Blanquet 1979, Rodwell 1991, Gillet 2000, Mueller-Dombois \& Ellenberg 2002, Chytrý \& Otýpková 2003, Sitte et al. 2004). Si se tiene en cuenta esta recomendación, se tendría que algo más del $82 \%$ de los inventarios florísticos publicados previamente a la realización del presente estudio han sido efectuados en superficies cuyo tamaño sería insuficiente para garantizar que el número de taxones presentes fuese representativo del ambiente ecológico caracterizado.

En el caso que nos ocupa, la representación gráfica de la relación entre el tamaño del área de muestreo y el número de especies vegetales presentes en cada inventario (Figura 4) muestra la existencia, de una correlación de tipo logarítmica entre ambos parámetros, comportamiento bien conocido desde hace tiempo en el ámbito de la ecología vegetal (Mueller-Dombois \& Ellenberg 2002). El resultado de este análisis revela que la mayoría de los inventarios en los que se computan 50 o más taxones de plantas vasculares han sido obtenidos en áreas de inventario iguales o superiores a $300 \mathrm{~m}^{2}$, mientras que los más pobres (25 sp. o menos) han sido tomados mayoritariamente en áreas de muestreo iguales o inferiores a $100 \mathrm{~m}^{2}$. En el caso de los inventarios inéditos aquí aportados (Figuras 3c y 4), más del $80 \%$ han sido realizados en unidades de muestreo de superficie igual o superior a $250 \mathrm{~m}^{2}$.

Un tercer aspecto que creemos importante para comprender la interpretación que se ha realizado hasta el momento sobre la variabilidad florística y fitocenótica de los bosques riparios de aliso en el territorio estudiado es el desigual reparto geográfico con el que se han efectuado los muestreos. Esta cuestión también ha sido discutida en trabajos anteriores a propósito de la caracterización de otros tipos de bosques presentes en el extremo NW Ibérico 
(Rodríguez Guitián 2010b) y, desde nuestro punto de vista, es quizás la principal causa de descripción de comunidades vegetales cuya composición florística "teórica" difiera más con respecto a lo que se puede reconocer en la naturaleza. En este caso, la información recopilada muestra la ausencia casi total de datos florísticos sobre alisedas riparias en el territorio que se extiende entre las desembocaduras de los ríos Masma y Eume ( $\mathrm{N}$ de Galicia), a pesar de que en el trabajo de referencia de Amigo et al. (1987) suponía, aproximadamente, un tercio del área geográfica abarcada por dichos autores. Ello ha conllevado la generalización a un ámbito biogeográfico de considerable extensión de una caracterización florística de las alisedas riparias fundamentada en un muestreo "parcial" e insuficiente de estos bosques, obviando posibles variaciones territoriales de su composición botánica debidas a causas como la propia corología de los taxones presentes, aspectos bioclimáticos, la influencia de los variados sustratos litológicos existentes en la morfología y dinámica de los cauces y las eventuales diferencias nutricionales de los suelos de las riberas, etc.

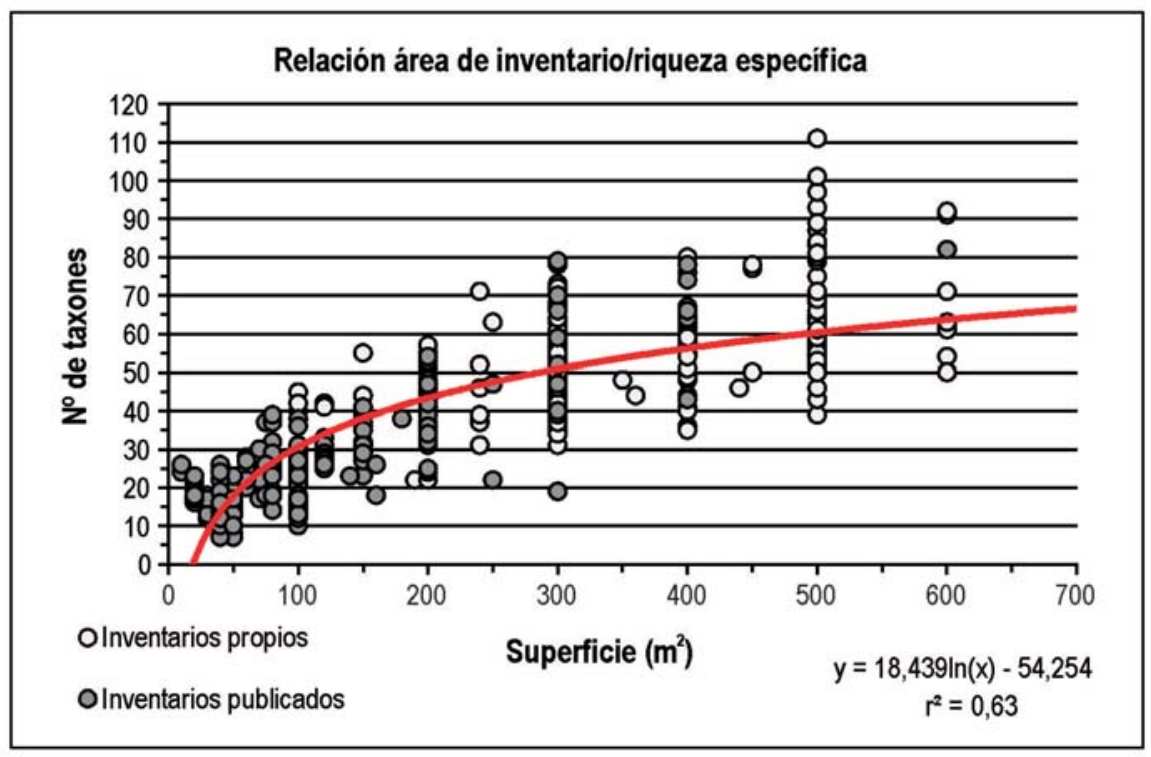

Figura 4.- Representación gráfica del número de especies presentes en cada inventario con relación a su respectiva área de muestreo $\left(\mathrm{m}^{2}\right)$ para las alisedas riparias estudiadas. En conjunto, las muestras tomadas de referencias bibliográficas (círculos negros) presentan un menor número de especies y superficie de inventariación que las aportadas en este trabajo (círculos blancos)

Todos estos aspectos contribuyen a explicar, en nuestra opinión, las dificultades comentadas al inicio de este trabajo con relación a los problemas de identificación fitosociológica de las alisedas riparias de numerosos tramos fluviales del $\mathrm{N}$ de Galicia y W de Asturias haciendo uso de los criterios florísticos propuestos por Amigo et al. (1987). También podrían explicar algunas propuestas interpretativas para este amplio tipo de bosques realizadas desde ámbitos no fitosociológicos, como la realizada por Lara et al. (2004), en las que se mezcla información bibliográfica con otra obtenida mediante diferentes metodologías.

\section{La transición florística cantábrica occidental/galaico- portuguesa en las alisedas estudiadas}

Expuestas las consideraciones anteriores, pasamos a comentar los resultados obtenidos acerca de la distribución encontrada para los grupos de especies que se han venido utilizando como diferenciales de las asociaciones descritas.

En síntesis, la diferenciación florística de las alisedas cántabro-atlánticas realizada por Amigo et al. (1987) se fundamenta en la distribución de dos grupos de especies de plantas vasculares a lo largo de un gradiente geográfico E-
W que abarca la totalidad de territorios cántabro-atlánticos, siguiendo las combinaciones que se muestran en la Tabla 1. Por un lado, el fresno común (Fraxinus excelsior), unido a Valeriana pyrenaica y un grupo de especies nemorales exigentes en nutrientes y humedad ambiental características de los órdenes Fagetalia sylvaticae y Populetalia albae (Carex remota, Circaea lutetiana, Festuca gigantea, Stachys sylvatica), serían más frecuentes y abundantes hacia el E y tenderían a desaparecer progresivamente hacia el W, especialmente al W y $S$ del Golfo Ártabro; por otro, diversas especies frecuentes en ambiente húmedos del extremo occidental ibérico (principalmente higrófitos que crecen adecuadamente sobre suelos no demasiado ricos en bases), cuya presencia se reduciría paulatinamente y de manera desigual hacia el $\mathrm{E}$ de los territorios cántabro-atlánticos ibéricos (Carex elata subsp. reuteriana, Fraxinus angustifolia, Galium broterianum, Osmunda regalis), situándose su límite oriental de distribución en la desembocadura del Río Nalón. En medio de estos gradientes florísticos contrapuestos se encontrarían los territorios cantábricos occidentales, en los que las alisedas se caracterizarían por la presencia simultánea de diversas especies características del orden Fagetalia sylvaticae y plantas como Carex reuteriana, Osmunda regalis y Valeriana pyrenaica. Estas tres últimas 
especies estarían prácticamente ausentes de los ríos más orientales (alisedas cántabro-vascónicas), mientras que las citadas más arriba, junto a Fraxinus excelsior, se extinguirían progresivamente entre la cuenca baja del Río Eume y la del Ulla, dentro ya del dominio establecido para la alisedas galaico-portuguesas.

Frente a esta propuesta, los resultados obtenidos en nuestros trabajos muestran una situación bastante más compleja, que se podría sintetizar en los siguientes puntos:

a) en primer lugar, Valeriana pyrenaica es un megaforbio frecuente a lo largo de los cauces fluviales de la cordillera cántabro-pirenaica con irradiaciones septentrionales hacia el litoral cantábrico y meridionales en la parte septentrional de Sistema ibérico y las montañas Galaico-Durienses, de aparición escasa en el extremo NW de la provincia de Lugo y muy puntual en la de A Coruña, de donde únicamente se le conoce, si se exceptúa la cita de esta especie publicada por F. Bellot (1951) hace más de 70 años ("valles de la elevada penillanura que se extiende desde Órdenes hacia Carballo"), de algunos valles de la Serra da Capelada y la cabecera del Río Mera (Tabla 2, Figura 5a). Su progresiva desaparición hacia su límite occidental de distribución tiene lugar a lo largo del área no prospectada por Amigo et al. (1987), lo que ha dado pie a problemas interpretativos derivados de su ausencia en una proporción apreciable de las cuencas fluviales del norte de Lugo y A Coruña.

b) paralelamente, la mayor parte del resto de las especies consideradas por Amigo et al. (1987) como diferenciales de las alisedas cantábricas occidentales y laciano-ancaresas de la Valeriano-Alnetum frente a las galaico-portuguesas de la Senecioni-Alnetum, mantienen su presencia en los bosques aquí tratados hasta bastante más al sur de lo establecido por estos autores. Para varias de estas especies (Circaea lutetiana, Carex remota, Fraxinus excelsior) su presencia comprende, al menos, las cuencas de los ríos Mandeo, Anllóns, Xallas, Tambre, Ulla y el curso medio y bajo del Miño, así como las de algunos pequeños ríos pontevedreses que desembocan en las Rías Baixas (Umia, Oitavén). Dado que, en algunos casos, estos bosques meridionales carecen de los taxones diferenciales de las alisedas galaico-portuguesas indicados por Amigo et al. (1987) (Fraxinus angustifolia, Galium broterianum), podría plantearse, en aplicación de los criterios utilizados por estos autores, su asimilación a las alisedas de la Valeriano-Alnetum. En el caso concreto de Fraxinus excelsior, su área de distribución conocida en el extremo noroccidental ibérico (Figura $5 b$ ), lejos de extinguirse de manera brusca al sur de la cuenca del río Ulla, se mantiene de manera discontinua a lo largo del área sublitoral de las Rías Baixas y el tramo final del Río Miño. De la misma manera, se ha constatado la presencia de esta especie arbórea en las márgenes de ríos de numerosas localidades interiores gallegas cuya vegetación se ha venido asimilando con alisedas de la Senecioni-Alnetum, tanto en cuencas de tributarios del río Miño (Izco et al. 1994) como del Sil (Romero Buján 1993).

A la vista de esta situación, parece difícilmente sostenible desde el punto de vista florístico y escasamente resolutivo desde el punto de vista fitosociológico, el mantener la existencia de una situación "ecotónica" entre las alisedas galaico-portuguesas y las cantábricas y orocantábricas occidentales fundamentada en la presencia del fresno común. Ello implicaría admitir que dicho "ecotono" o contacto biogeográfico se extendería prácticamente desde la desembocadura del Río Eume, en el Golfo Ártabro, hasta el norte de Portugal, comprendiendo alrededor de la mitad del área de distribución atribuida a esta asociación de alisedas riparias (cuyo límite meridional se encontraría al sur de Oporto), con lo que la subasociación "fraxinetosum excelsioris" pasaría de ser una "particularidad florística" de un ámbito geográfico relativamente limitado, a un fenómeno que se podría observar en más de la mitad del territorio dentro del que se encontraría esta asociación vegetal, perdiendo por completo su significado fitosociológico original. Llegados a este punto, estaría por dilucidar cómo y a lo largo de qué territorio tendría lugar el paso de las alisedas cantábricas occidentales y orocantábricas de la Valeriano-Alnetum a las galaico-portuguesas de la Senecioni-Alnetum.

c) otra circunstancia que resulta especialmente llamativa es la total ausencia de Narcissus cyclamineus, junto a otros geófitos, en las tablas florísticas de la Senecioni-Alnetum de Amigo et al. (1987), hecho derivado, muy posiblemente, de la realización de los inventarios florísticos en momentos de su ciclo vital no apropiados para su detección en el campo. La ausencia del narciso mencionado en dichos inventarios contrasta con la gran cantidad de citas y pliegos de herbario sobre los que se ha ido construyendo el conocimiento de su corología y ecología desde hace más de cien años (Moreno Sáez \& Saínz Ollero 1992), así como con la información aportada por Casaseca (1959) en sus inventarios de bosques de ribera de las inmediaciones de Santiago de Compostela, área comprendida dentro del ámbito biogeográfico que Amigo et al. (1987) establecen para la asociación comentada. Si nos atenemos a la información proporcionada por Pino et al. (2009) en su revisión sobre la distribución conocida y ecología de este endemismo del NW Ibérico, se trataría de una especie frecuente en bosques húmedos (alisedas riparias y pantanosas, robledales de vega en contacto con los anteriores) y praderas higrófilas de la vertiente atlántica galaico-portuguesa, con presencia generalizada en este territorio desde Aveiro (Portugal) hasta la cuenca del Río Mandeo (A Coruña) (Figura 5a cuadrados naranja y rombos morados). Nuestros datos confirman que Narcissus cyclamineus es una especie frecuente en las alisedas riparias de la cuenca baja y media del Río Mandeo, desde su desembocadura hasta un poco más arriba de su unión con el Río Deo, cerca de la localidad de Teixeiro (Curtis, A Coruña). Más hacia el Norte, no conocemos poblaciones de esta especie en la cuenca del Río Lambre y solamente hemos localizado dos enclaves en los que está presente en la cuenca baja del Río Baxoi, no superando los $100-150$ m de altitud.

Posiblemente, el mayor grado de continentalidad y frío invernal que presentan las tierras altas coruñesas del entorno del Golfo Ártabro y las sierras que conforman la Dorsal Gallega (Rodríguez Guitián \& Ramil-Rego 2007) sea un factor limitante para el crecimiento en altura de esta especie de carácter termófilo, que raramente supera los 450 
$\mathrm{m}$ de altitud a lo largo de las áreas de cabecera de los ríos Tambre y Ulla. Traspasada la Dorsal Gallega hacia el oriente, esta amarilidácea tan sólo se conoce de algunos tramos fluviales abrigados, y hasta cotas no superiores a los $500 \mathrm{~m}$, en la cuenca del Río Ferreira, tributario del Miño (Figura 5a), de dónde fue citada por Fagúndez (2003) a 464 $\mathrm{m}$ de altitud. Este temperamento termófilo explicaría la ausencia absoluta de esta bulbosa de la cuenca alta del Río Miño, de condiciones más continentales, cuestión que ya había sido vaticinada por Laínz (1966). A pesar de ello, Izco (1987) llegó a proponer una combinación fitosociológica para los robledales climatófilos de Terra Chá con la participación de este taxón (Narcisso cyclaminei-Quercetum roboris nomem nudum). Propuesta que, aunque inválida de todo punto desde una perspectiva fitosociológica, fue incorporada a la base de datos del proyecto Corine Biotopes (CE 1991) y mantenida en otras clasificaciones europeas posteriores de tipos de hábitats, como Paleartic Habitats typology (Devillers \& Devillers-Terschuren 1996) o EUNIS Habitat Classification (EEA 2017), dando por hecho que el geófito comentado habría de estar presente en esta parte de Galicia.
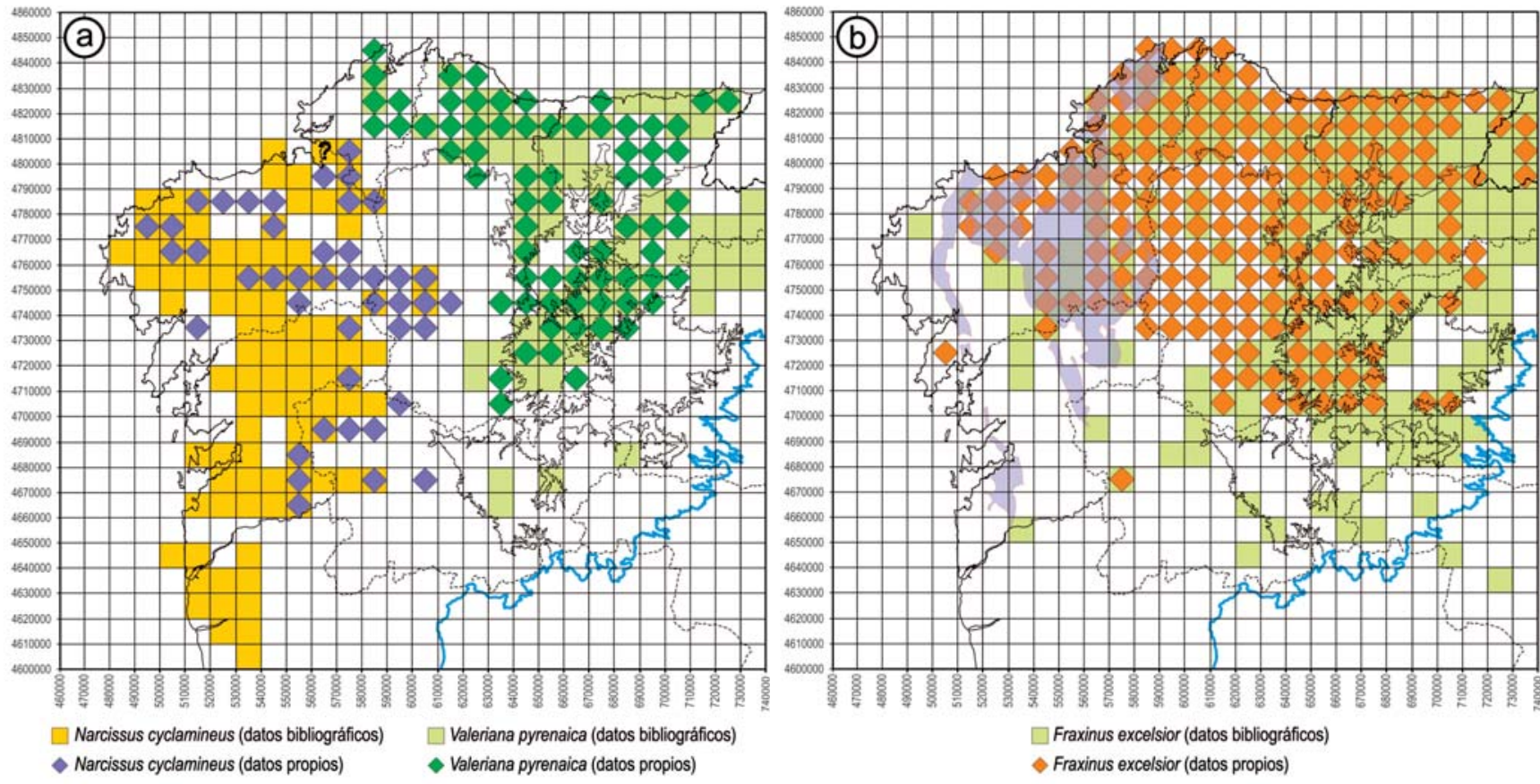

Figura 5.- a: Distribución conocida de Valeriana pyrenaica (se ha prescindido de la cita de las proximidades de Carballo de Bellot [1951]) y Narcissus cyclamineus (se ha prescindido de la cita de Laínz [1966] de las proximidades de Viveiro) en el área de estudio. Elaborado a partir de: Pino et al. (2009), Proyecto Anthos (2017), SIVIM (2017) y datos propios. b: Distribución conocida de Fraxinus excelsior en el área de estudio. En sombreado gris se delimitan los afloramientos de rocas metamórficas silíceas básicas y ultrabásicas. Elaborado a partir de: Proyecto Anthos (2017), SIVIM (2017) y datos propios.

Por otra parte, a lo largo de los diversos trabajos que hemos efectuado en el área litoral gallega situada al norte de la desembocadura del Río Eume no hemos encontrado ninguna población de este narciso, en concordancia con lo constatado por Fagúndez (2011) para el municipio de Ferrol (A Coruña). Esta ausencia contrasta con la cita publicada hace más de 50 años por Laínz (1966) en las proximidades de Viveiro (Lugo), a raíz de una comunicación personal del profesor B. Casaseca y que, por lo que sabemos, carece de pliego de respaldo. Si se exceptúa esta última supuesta localidad norteña extrema no confirmada a día de hoy, el área septentrional de presencia generalizada y habitual de esta especie se ajusta básicamente a la delimitación de la frontera biogeográfica establecida para los sectores Galaico-Portugués y Galaico-interior con el subsector
Cantábrico occidental (Sector Galaico-Asturiano) por Rodríguez Guitián \& Ramil-Rego (2008) (Figura 5b). Por esta razón, creemos que Narcisus cyclamineus debería considerarse como una especie característica y diferencial de las alisedas galaico-portuguesas frente a las cantábricas occidentales y orocantábricas.

La aceptación de esta línea argumental lleva a considerar como artificiosa la separación de los bosques ribereños de territorios portugueses miñenses litorales (Narcisso cyclaminei-Alnetum glutinosae) frente a los de la SenecioniAlnetum planteada por Honrado et al. (2002), pues, al decaer el carácter de taxón diferencial de $N$. cyclamineus, ésta se sustentaría exclusivamente en la presencia en los primeros de Hedera helix subsp. canariensis y Thalictrum 
speciosissimum. No obstante, hay que señalar que, si se atienden los criterios taxonómicos de identificación propuestos por Valcárcel et al. (2002) en su revisión del género Hedera, el taxón de hiedra indicado por los autores portugueses no estaría presente dentro del ámbito ibérico, por lo que habría de suponerse que, en realidad se trate de $H$. hibernica, planta cuya presencia en el tramo bajo del Río Miño y en la cuenca del Limia ha sido reconocida por Amigo et al. (2009) y Honrado et al. (2007), respectivamente; además, la segunda de las plantas indicadas sería de aparición más bien esporádica en dichos bosques, a juzgar por la composición florística mostrada en la tabla original de 8 inventarios sobre la que se sustenta la descripción de dicha asociación. El resto de taxones inicialmente propuestos por los autores portugueses citados para diferenciar ambas asociaciones (Fraxinus angustifolia, Laurus nobilis, Ruscus aculeatus, Arum italicum y el propio Narcissus cyclamineus) carecen realmente de ese carácter discriminante, pues todos ellos se pueden considerar frecuentes en las alisedas riparias de la fachada atlántica de Galicia situada al S del Golfo Ártabro. En este sentido, Costa et al. (2012) y Biurrun et al. (2016) han publicado sendos trabajos de actualización sintaxonómica en los que proponen la sinonimización de Narcisso-Alnetum con la Senecioni-Alnetum, aunque sin aportar ningún tipo de justificación al respecto. Los datos que aquí se presentan dejan claro, en nuestra opinión, que la asociación Narcisso cyclaminei-Alnetum glutinosae debe considerarse, efectivamente, como un sinónimo nomenclatural de la Senecioni-Alnetum

La delimitación precisa de la distribución de las alisedas de la Senecioni-Alnetum a lo largo de la cuenca media del Río Miño y del tramo final del Sil está por resolver. Si tomamos como guía la distribución conocida de Narcisus cyclamineus, éstas se extenderían a lo largo de la cuenca del Río o Avia, la comarca del Ribeiro y la hoya de Ourense y abarcarían una parte extensa de las cuencas que nacen a lo largo de la vertiente oriental de la Dorsal Gallega, entre Os Peares y la desembocadura del Río Ferreira en el Miño. La dilucidación del límite oriental de estos bosques en el tramo final del Río Sil es una tarea particularmente difícil de aclarar, habida cuenta del nivel de degradación que la construcción de grandes embalses han impuesto sobre las condiciones ecológicas de su cauce y el de algunos de sus principales tributarios (Mao, Návea, Xares, Bibei) y que han sido responsables, igualmente, de la desaparición de otros tipos de bosques de ribera en esta y otras cuencas fluviales de Galicia (Amigo 2005, Ramil-Rego et al. 2010). En este caso, la hipótesis a testar sería su substitución por la subasociación con fresno eurosiberiano de las alisedas carpetano-leonesas (Galio broteriani-Alnetum glutinosae subas. fraxinetosum excelsioris), cuya existencia en diversas localidades del cuadrante SE de Galicia se ha venido admitiendo desde hace varias décadas (Díaz González et al. 1986, Izco et al. 1999). Menos dificultades debería entrañar la verificación de la identidad fitosociológica de las alisedas riparias que pueblan la cuenca Alta del Río Miño, para determinar si pertenecen a la asociación Senecioni-Alnetum, tal y como plantearon Izco et al. (1994).
La transición florística cantábrica occidental/ovetense en las alisedas estudiadas

Durante la realización de nuestros trabajos de campo en el extremo oriental del área de estudio hemos hecho un seguimiento análogo al descrito en el apartado anterior a aquellas especies que Amigo et al. (1987) establecieron como diferenciales de las alisedas galaico-asturianas de la Valeriano-Alnetum y las cántabro-euskaldunas de la Hyperico-Alnetum. La información florística contenida en nuestros inventarios muestra que cuatro de las cinco especies consideradas como diferenciales de las alisedas cántabro-euskaldunas (Equisetum telmateia, Helleborus viridis subsp. occidentalis, Saxifraga hirsuta y Stegnogramma pozoi), están presentes en las alisedas galaico-asturianas occidentales estudiadas, siendo Lamiastrum galeobdolon el único taxón de los inicialmente propuestos como diferenciales por Amigo et al. (1987) que está totalmente ausente de este territorio y que se comportaría, consecuentemente, como especie exclusiva (diferencial) de las alisedas ovetenses.

La interpretación estricta de los criterios florísticos de Amigo et al. (1987) llevaría a considerar que aquellos tramos de alisedas cantábricas y orocantábricas occidentales en las que se ha documentado la presencia de una o varias de las especies anteriormente citadas se deberían incluir en la subasociación osmundetosum regalidis de las alisedas cántabro-euskaldunas, en la línea de lo planteado por dichos autores para el caso de diversos inventarios tomados por Díaz González (1975) en las cuencas asturianas de los ríos Orio y Posadas. Con la información que actualmente disponemos, esto equivaldría a afirmar que los bosques de la Hyperico-Alnetum osmundetosum se extenderían, más al W de la desembocadura del Río Nalón, no sólo a lo largo de diversas localidades de los concejos asturianos de Cudillero y Valdés, por los que circulan los tramos de los ríos inventariados por Díaz González, sino que alcanzarían el extremo septentrional de la provincia de A Coruña (Serra da Capelada) y el área centro-oriental lucense (sierras de Ancares y O Courel).

Varios motivos nos llevan a plantear que esta interpretación no parece la más adecuada para estas situaciones. Quizás, el más obvio sea que no siempre la presencia de estas especies meso-eutrofas coincide con la existencia de afloramientos de rocas carbonatadas en los territorios galaico-asturianos y orocantábricos occidentales. De hecho, en los escasos tramos fluviales en los que se puede reconocer la existencia de dicho roquedo en los bordes de los ríos (inventarios marcados con * en la tablas $\mathrm{V}$ y VII del Anexo), éste aflora conformando estratos de poca potencia, de manera que, aunque los suelos puedan mostrar puntualmente un mayor grado de trofía, las aguas fluviales pobres en carbonatos y el aporte continuado de sedimentos de origen silíceo desde las vertientes circundantes, amortiguan su influencia sobre la flora constituyente de estos bosques de ribera. Por otro lado, la subasociación osmundetosum regalidis de la Hyperico-Alnetum se describió para reunir los aspectos menos eutrofos de las alisedas riparias cántabro-euskaldunas cuando, debido a la existencia local de sustratos silíceos, en ellas aparecen 
especies típicas de bosques de ribera asentados sobre litologías ácidas, como Osmunda regalis y Carex reuteriana, pero manteniendo la presencia de las especies eutrofas características de la asociación (Amigo et al. 1987, ver Tabla 2, columnas 12 a 14b). Sin embargo, la presencia simultánea de estos dos grupos de especies no se observa en los escasos tramos de alisedas cantábricas occidentales y orocantábricas que atraviesan roquedos carbonatados dentro de nuestra área de estudio. Todo ello nos lleva a considerar que la presencia en algunos tramos de alisedas galaico-asturianas y orocantábricas occidentales de ciertas especies meso-eutrofas frecuentes en las alisedas de la Hyperico-Alnetum debe ser considerada dentro de la variabilidad florística de los bosques de la asociación Valeriano pyrenaicae-Alnetum glutinosae y no como representaciones occidentales de dichas alisedas cántabroeuskaldunas.

Nuevos criterios florísticos de diferenciación de las alisedas (sub)litorales del extremo NW ibérico

Conocida en detalle la distribución y comportamiento ecológico de las especies establecidas por Amigo et al. (1987) para discriminar las alisedas estudiadas, la confrontación de la información previamente publicada con la aportada en este trabajo muestra que, en la mayoría de los casos, dichos taxones carecen del carácter diferencial que en su día se les atribuyó. Por el contrario, el manejo de una más abundante y mejor distribuida información sobre la composición florística de estos bosques pone de manifiesto la existencia de otros conjuntos de plantas, principalmente herbáceas, que pueden considerarse como discriminantes frente a los bosques riparios de aliso pertenecientes a las dos asociaciones con las que establecen contacto hacia el W y el E.

En cuanto a la diferenciación de las alisedas cantábricas occidentales y altonarceense-ancaresas (ValerianoAlnetum) de las galaico-portuguesas (Senecioni-Alnetum), además de Valeriana pyrenaica, se ha constatado la presencia en las primeras de algunas especies que, hasta el momento, no se han encontrado en las segundas, como Adenostyles alpina, Avenella flexuosa, Cytisus commutatus, Dryopteris aemula, Helleborus foetidus, Helleborus viridis subsp. occidentalis, Lastrea limbosperma, Pulmonaria longifolia, Sanicula europaea, Saxifraga hirsuta, Saxifraga x polita, Scrophularia alpestris, Symphytum tuberosum (Tabla 2). En sentido inverso, el número de taxones que se muestran como exclusivos de la Senecioni-Alnetum frente a Valeriano-Alnetum es sensiblemente menor, pues a la vista de la información aquí reunida, tan sólo podrían citarse como tales Narcissus cyclamineus, Galium broterianum, Anemone albida y Echium lusitanicum.

Por otro lado, el análisis de la información aquí presentada permite establecer que un cierto conjunto de especies características de la clase Carpino-Fagetea, indicadoras de una mayor trofía edáfica, frecuentes en las alisedas de la Valeriano-Alnetum, aparecen en numerosos ríos que vierten al Oceáno Atlántico al $\mathrm{S}$ de la desembocadura del Río Eume de una manera sensiblemente más frecuente que lo considerado por Amigo et al. (1987). Así, se pueden encontrar en estos bosques árboles como Acer pseudoplatanus, o Ulmus glabra junto a un conjunto bastante amplio de plantas herbáceas características del orden Fagetalia sylvaticae (Allium ursinum, Carex sylvatica, Conopodium majus, Lysimachia nemorum, Melica uniflora, Mercurialis perennis, Ornithogalum pyrenaicum, Poa nemoralis, Potentilla montana, Potentilla sterilis, Primula acaulis, Ranunculus tuberosus, Stachys sylvatica, Veronica montana). Con frecuencia, en estas mismas alisedas son frecuentes taxones higro-esciófilos como Allium scorzonerifolium, Arum italicum, Carex remota, Chrysosplenium oppositifolium, Myosotis martini y Oxalis acetosella (comparar columnas 1 y 4 con 2, 3 y 5, Tabla 2). En nuestra opinión, la distribución de este conjunto de taxones está asociada a la presencia en este amplio territorio geográfico de una topografía suave, favorable para la alteración en profundidad de unos sustratos mayoritarios (esquistos biotíticos pobres en cuarzo, rocas básicas y ultrabásicas) relativamente ricos en nutrientes, de texturas finas y con una elevada capacidad de retención de agua (cf. Calvo de Anta \& Macías Vázquez 2001), lo que favorecería la presencia de dichos vegetales en los bosques estudiados hasta localidades situadas en el centro-sur de Galicia (Figura 2).

En el caso de las alisedas presentes a lo largo de la Cornisa Cantábrica, el examen de la información manejada en este estudio revela que las alisedas cantábricas centroorientales de la Hyperico-Alnetum se diferencian florísticamente de las de la Valeriano-Alnetum por la presencia de un amplio grupo de especies que, además de Lamium galeobdolon, incluye, entre aquellas que alcanzan frecuencias de aparición más elevadas, Salix alba, Hedera helix, Lathraea clandestina y Ligustrum vulgare, a las que habría que añadir algunas otras de aparición más esporádica, pero con un marcado significado biogeográfico cantábrico centro-oriental, ya que están ausentes de manera espontánea en las alisedas riparias de los territorios litorales y sublitorales situados más al $W$ de la desembocadura del Río Nalón. Entre ellas se podrían citar Buxus sempervirens, Carex distans, Carex hirta, Carex strigosa, Equisetum palustre, Equisetum ramosissimum, Equisetum x moorei, Erica lusitanica, Juncus inflexus, Primula elatior, Quercus ilex, Rosa sempervirens, Salix discolor, Salix eleagnos, Sambucus ebulus, Silene nemoralis o Viola sylvestris (columnas 13 y 14, Tabla 2).

En sentido inverso, el conjunto de especies presentes en las alisedas de la Valeriano-Alnetum que no aparecen en las de la Hyperico-Alnetum incluye un extenso repertorio de plantas vasculares, unas comunes con las alisedas galaicoportuguesas de la Senecioni-Alnetum, como Allium scorzonerifolium, Allium victorialis, Angelica major, Aquilegia dichroa, Arbutus unedo, Betula pubescens, Caltha palustris, Carex laevigata, Ceratocapnos claviculata, Conopodium majus, Deschampsia subtriflora, Dryopteris aemula, Erica arborea, Euphorbia hyberna, Hieracium umbellatum, Luzula forsteri, Narcissus asturiensis, Narcissus triandrus, Omphalodes nitida, Peucedanum lancifolium, Physospermum cornubiense, Pyrus cordata, Quercus pyrenaica, Saxifraga spathularis, Solidago virgaurea, 
Vaccinium myrtillus, Viola palustris; mientras que otras (Adenostyles alpina, Avenella flexuosa, Cardamine impatiens, Cytisus commutatus, Erica mackaiana, Helleborus foetidus, Lathraea squamaria, Quercus petraea, Quercus petraea $\times$ Q. robur, Salix fragilis, Saxifraga lepismigena, Scrophularia alpestris, Valeriana pyrenaica) son exclusivas.

Admitidas estas puntualizaciones, el dominio territorial de las alisedas cantábricas occidentales (as. Valerianopyrenaicae-Alnetum glutinosae) se extendería, hacia el W, por la totalidad de la cuenca del río Eume y las partes altas y medias (hasta los 150-200 m de altitud) de las de los ríos Baxoi y Lambre, así como la del Cambás, tributario del Río Mandeo, entre su desembocadura en el Río Mandeo y los $450 \mathrm{~m}$ de altitud (Figura 2), pues en cotas superiores las alisedas se ven sustituidas por otros tipos de bosques riparios, como fresnedas, abedulares o avellanedas (Rodríguez Guitián et al. 2010, 2017). Entre el límite S de la Cuenca del Río Eume y la cabecera del Río Mandeo, por debajo de los $250 \mathrm{~m}$ de altitud, los bosques de ribera muestran un carácter transicional entre las combinaciones florísticas adscribibles a la Valeriano-Alnetum y la Senecioni-Alnetum que se evidencia por la aparición conjunta de especies características de la primera, como Festuca gigantea o Chaerophyllum hirsutum junto a Narcissus cyclamineus, taxón diferencial de la segunda asociación citada.

En el extremo opuesto, el límite oriental de estos bosques riparios de aliso vendría a coincidir con la traza que describen los afloramientos de materiales carbonatados paleozoicos y mesozoicos que flanquean la margen izquierda del tramo bajo del Río Nalón, entre las localidades asturianas de Muros del Nalón y Pravia, remontando, a continuación, por la cuenca del Río Narcea hasta, aproximadamente, su unión con el Río de Junqueras, al SW de la localidad de Tineo. Hacia el E y SE del territorio aquí tratado, Rodríguez Guitián (2004) ha recogido inventarios de alisedas cuya composición florística es asimilable a la de esta asociación en algunas localidades de la cuenca alta del Río Miño, a las que hay que sumar otras más aportadas en este trabajo. No obstante, queda pendiente de precisar de qué manera se realiza, si es que realmente tiene lugar en la cuenca alta del Río Miño, la transición entre esta asociación y la Senecioni-Alnetum, cuestión sobre la que no se ha vuelto a debatir en el plano científico desde que Izco et al. (1994) plantearon la presencia de esta última comunidad en las inmediaciones de la capital lucense en base a una escueta tabla de tres inventarios caracterizados por una extraordinaria pobreza florística (entre 12 y 14 taxones) efectuados en superficies de inventario de 40 a $100 \mathrm{~m}^{2}$.

Interpretación sintaxonómica de la variabilidad florística de los bosques estudiados

Dentro de las alisedas galaico-asturianas, como en las otras dos asociaciones objeto de comentario en este trabajo, se han propuesto unidades sintaxonómicas de rango inferior al de asociación para encuadrar diversas combinaciones florísticas particulares (Amigo et al. 1987).
A pesar de que en todos los casos la unidad utilizada ha sido la subasociación, las causas aducidas para justificar la descripción de dichas unidades son de naturaleza heterogénea y tienen una manifestación territorial (biogeográfica) de muy diferente magnitud. Así, la subasociación fraxinetosum excelsioris de la SenecioniAlnetum se propuso, como ya se ha comentado, como expresión de una situación transicional o ecotónica, desde el punto de vista biogeográfico, entre los bosques de la subasociación típica (Senecioni-Alnetum alnetosum glutinosae) y los de la Valeriano-Alnetum. Sin embargo, en otros casos se han aducido motivos paleoambientales (relictismo) para justificar la descripción de una unidad sintaxonómica de rango similar (subas. fraxinetosum angustifoliae) que acogería las alisedas galaico-asturianas del tramo bajo del Río Navia (Lugo-Asturias) en las que están presentes determinados fanerófitos frecuentes en comunidades vegetales riparias más meridionales, como Fraxinus angustifolia, F. excelsior x F. angustifolia o Salix salviifolia. En último lugar, en el caso de las alisedas ovetenses y cántabro-vascónicas ya hemos señalado el recurso a factores relacionados con la naturaleza química del sustrato para explicar las peculiaridades florísticas de las alisedas cántabro-euskaldunas incluidas en la subasociación osmundetosum regalidis.

En diversos trabajos previos (Rodríguez Guitián 2004, 2006, 2010b; Rodríguez Guitián et al. 2009a, 2009b) hemos venido defendiendo, siguiendo a autores como Géhu (1998) y en aras a alcanzar una generalización del método fitosociológico, que la descripción de sintaxones de rango inferior al de asociación (subasociación, variante, subvariante, facies, etc.) debe de estar sustentada en fenómenos o factores causales que se mantengan a lo largo de la totalidad del área geográfica en la que se reconozca la presencia de la unidad fitosociológica elemental a la que se subordinan. Por extensión, las unidades de rango inferior reconocibles en asociaciones vicariantes que se distribuyen a lo largo de territorios biogeográficos contiguos deben de mantener una homología en cuanto a su tipología y relación con factores responsables de las variaciones florísticas que representan siempre que se mantengan las causas ambientales que las propician. Si se admite este planteamiento, parece evidente que es necesario realizar una adaptación de la correspondencia entre unidades fitosociológicas de rango inferior al de asociación (subasociaciones, variantes, subvariantes y facies) y los factores causales de las respectivas combinaciones florísticas que representan dentro de los bosques comentados, en la línea de lo mostrado en la Tabla 3.

Según esta propuesta, la descripción de subasociaciones en los bosques estudiados estaría justificada para discriminar los aspectos más ampliamente distribuidos territorialmente, en los que se observa la que presencia de especies termófilas, como Arbutus unedo, Asplenium onopteris, Bryonia dioica, Calystegia sepium, Carex pendula, Clematis vitalba, Humulus lupulus, Laurus nobilis, Phalaris arundinacea, Phyllitis scolopendrium, Rubia peregrina, Ruscus aculeatus, Smilax aspera, Solanum dulcamara o Tamus communis, indicadoras de los 
termotipos termotemplado y mesotemplado inferior (Díaz González \& Fernández Prieto 1994; Rodríguez Guitián \& Ramil Rego 2007), de aquellos otros, por lo general situados dentro del termotipo mesotemplado superior y, más raramente, supratemplado inferior, que carecen de tales especies.

\begin{tabular}{lll}
\hline Factor causal & Rango fitosociológico & Ejemplo \\
\hline Elemento bioclimático (termotipo) & subasociación & subas. termófila / mesófila / orófila \\
Elemento bioclimático (oceaneidad) & variante & variante hiperoceánica / semioceánica / suboceánica \\
Riqueza en nutrientes do solo & subvariante & variante oligotrofa / mesotrofa \\
Situaciones ecotónicas & facies & facies de transición \\
\hline
\end{tabular}

Tabla 3.- Correspondencia entre factores responsables de la variación florística de una asociación vegetal y los rangos fitosociológicos aplicados en el presente trabajo

En lo que a la asociación Valeriano pyrenaicae-Alnetum glutinosae se refiere, aceptando como válida la tipificación realizada por Amigo et al. (1987), la subasociación típica representaría a una comunidad termófila, por cuanto en el inventario tipo aparecen taxones como Carex pendula y Osmunda regalis, siendo la más extendida dentro del subsector Cantábrico occidental. No obstante, en ciertas áreas de la cabecera de los ríos Miño, Narcea, Navia, Lor y Sil existen alisedas que han sido incluidas en esta asociación pero que carecen de taxones termófilos, como se desprende del examen de las tablas de inventarios aportadas por Amigo (1984), Amigo et al. (1987), SilvaPando (1990) o Romero Buján (1993) y de los datos obtenidos por nosotros en algunas localidades de estos ámbitos geográficos (Anexo: Tabla VIII). Con frecuencia, estas "alisedas no termófilas" presentan taxones de cierto carácter "orófilo", raros o inexistentes en las áreas más térmicas, como Aconitum vulparia subsp. neapolitanum o Ranunculus platanifolius. Para estos casos, la aplicación de la correspondencia indicada en la Tabla 3 llevaría a plantear la existencia de una subasociación diferente de la típica caracterizada por dicha ausencia de taxones termófilos y la presencia de los orófilos citados. No obstante, esta opción precisaría, en nuestra opinión, de un estudio específico por lo que, por el momento, preferimos designarlas como "alisedas cantábricas occidentales y orocantábricas occidentales no termófilas" (columnas 12 a y 12 b, Tabla 2; Anexo: Tabla VII).

En relación a los inventarios de bosques de la SenecioniAlnetum y de la Hyperico-Alnetum manejados, en todos aquellos tomados por nosotros y en la inmensa mayoría de los de procedencia bibliográfica se documenta la presencia de especies termófilas, por lo que, al menos por el momento, creemos que no sería procedente la descripción de subasociaciones en base a causas termoclimáticas.

En otro nivel de segregación (variantes) deben situarse aquellos casos en que condicionantes bioclimáticos de otro orden causan ciertas modificaciones de la composición florística más extendida a lo largo del territorio que se considere. Es el caso de los mesoambientes de bioclima hiperoceánico o los microambientes de elevada humedad atmosférica, particularmente favorables para la incorporación a estos bosques de numerosos helechos marcadamente higrófilos y querenciosos por ambientes con poca oscilación térmica, como Culcita macrocarpa, Davallia canariensis, Vandenboschia speciosa, Hymenophyllum tunbrigense, Stegnogramma pozoi o Woodwardia radicans, entre otras especies. Estas situaciones son reconocibles dentro de las tres asociaciones de alisedas riparias aquí comentadas (Tabla 2).

Además, en el caso de la Valeriano-Alnetum, puede diferenciarse otra variante que incluiría aquellos otros enclaves caracterizados por una atmosférica más seca o contrastada termométricamente (localidades con bioclima de características suboceánicas) o un régimen hidrológico con un estiaje más marcado, que permiten la presencia de taxones como Fraxinus angustifolia, Fraxinus oxycarpa o Salix salviifolia. A la vista de la información aquí manejada, dicha variante está presente tanto en la parte media y baja de la cuenca del Río Navia (como inicialmente identificaron Amigo et al. 1987), como en diversas áreas de las cuencas bajas de los ríos Mao, Cabe y Lor, en la porción meridional de la provincia de Lugo, dentro de territorios biogeográficos galaico-interiores y valdeorreses en el sentido de Rodríguez Guitián \& Ramil Rego (2008), como irradiaciones a partir de los territorios próximos orocantábricos occidentales. Sin embargo, las especies arbóreas que caracterizan dicha comunidad no podrán utilizarse como diferenciales de un tipo de mesoambiente bioclimático local o particular en el caso de las alisedas galaico-portuguesas, pues en la fachada atlántica gallega y nor-portuguesa el clima dominante muestra ya de por sí una tendencia más marcada á la reducción de las precipitaciones (cf. Rodríguez Guitián \& Ramil Rego 2007), responsable de un estiaje mucho más evidente en los ríos, lo que favorecería una presencia generalizada de este conjunto de bioindicadores.

Las modificaciones locales de la composición florística de los bosques estudiados relacionadas con un incremento del contenido en nutrientes de los suelos debido a la presencia de substratos ricos en bases, ya fuese en forma de delgados afloramientos de rocas carbonatadas en el caso de las alisedas cantábricas occidentales, o a la de sustratos metamórficos o ígneos pobres en sílice, como esquistos biotíticos, anfibolitas, eclogitas, serpentinitas o gabros, en el de las alisedas galaico-portuguesas, se plasmaría, en el plano fitosociológico, en la definición de "subvariantes mesotrofas", dado el carácter mayoritariamente oligotrofo de los materiales litológicos presentes dentro del área biogeográfica aquí tratada. 
En último lugar cabría comentar el recurso al rango fitosociológico de facies para incluir las situaciones previamente comentadas en las que se observa la confluencia de especies características de las asociaciones Valeriano-Alnetum y Senecioni-Alnetum, registrada únicamente, dentro del ámbito de estudio considerado, en la cuenca del Río Mandeo (Figura 2; Tabla 2: columna 3; Anexo: Tabla III).
Como complemento y síntesis de la discusión planteada en los párrafos precedentes, se ha construido una clave dicotómica cuyo uso permite una fácil determinación de la identidad sintaxonómica (tanto a nivel de asociación como de unidades de rango inferior) de las diferentes combinaciones florísticas que pueden encontrarse dentro de las alisedas del territorio estudiado (Figura 6).

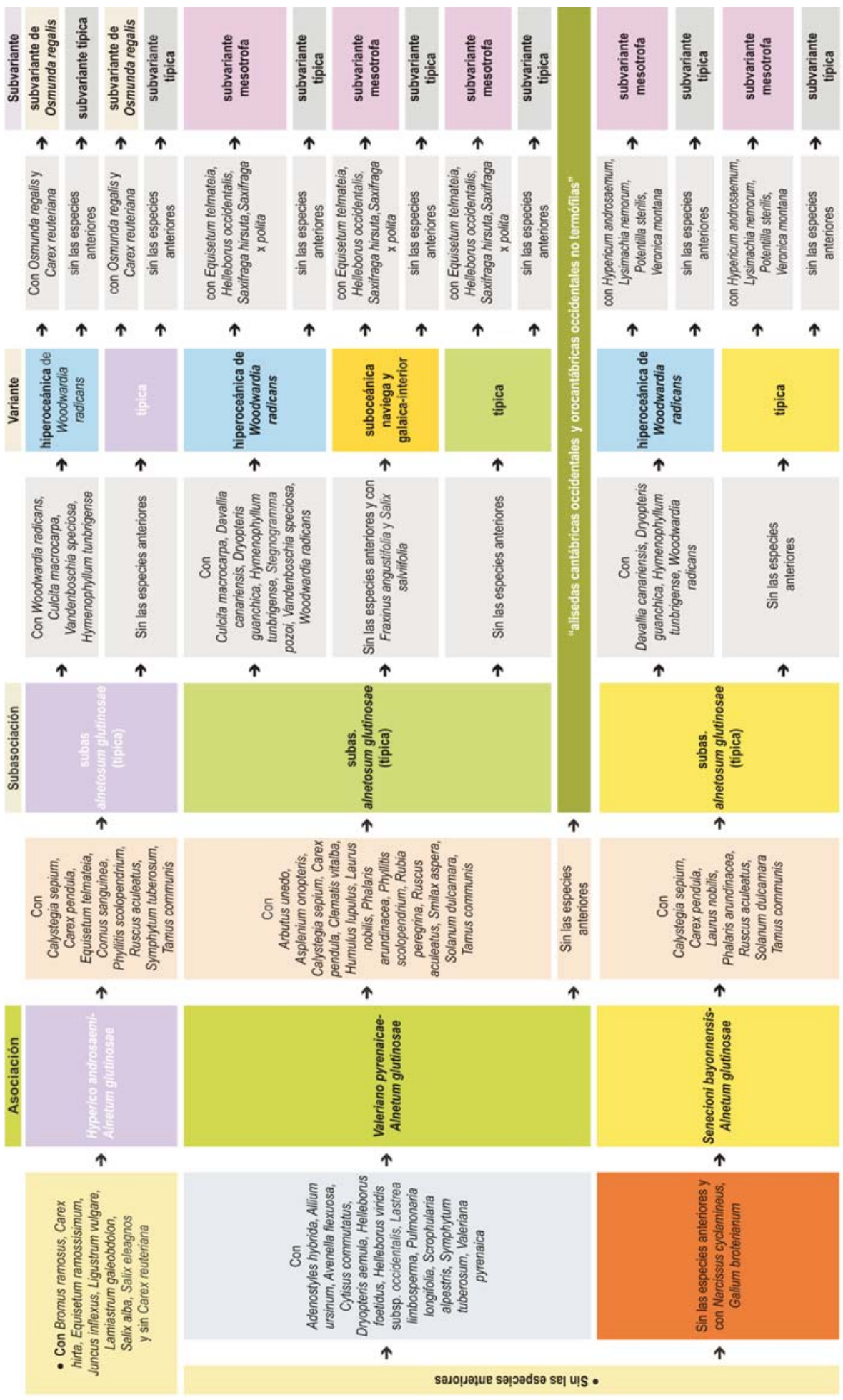

Figura 6.- Clave dicotómica para la discriminación de las asociaciones de alisedas del extremo noroccidental cántabroatlántico y de sus unidades subsintaxonómicas identificadas 


\section{Encuadre sintaxonómico}

Hasta hace muy poco tiempo, el esquema sintaxonómico utilizado para encuadrar los tipos de vegetación arbolada del ámbito noroccidental ibérico que crecen en los ambientes riparios incluía a todas las asociaciones descritas en dos alianzas (Alnion incanae, que reuniría las alisedas y fresnedas fluviales eurosiberianas, y OsmundoAlnion, que englobaría las alisedas y saucedas fluviales reófilas mediterráneas y transicionales galaicoportuguesas), dentro del orden Populetalia albae (clase Salici purpureae-Populetea nigrae) (Rivas-Martínez et al. 2011, Costa et al. 2013).

Sin embargo, como consecuencia de una reciente revisión de la tipología de unidades fitosociológicas europeas hasta el nivel de alianza realizada por Mucina et al. (2016), se ha considerado oportuno reubicar los bosques riparios cantábricos y orocantábricos de aliso dentro un orden descrito inicialmente para el ámbito alpino-centroeuropeo (Alno-Fraxinetalia excelsioridis Passarge 1968) y que a raíz de esta propuesta se extiende al ámbito biogeográfico atlántico de Europa, manteniendo el resto dentro del orden
Populetalia albae. Paralelamente, Biurrun et al. (2016) han estudiado las relaciones florísticas existentes entre los bosques riparios descritos del territorio ibérico, concluyendo que las presentes en el territorio cantábrico aquí tratado (Hyperico-Alnetum y Valeriano-Alnetum) deben de ubicarse en dicho orden Alno-Fraxinetalia excelsioridis, mientras que la asociación de distribución atlántica (Senecioni-Alnetum) se mantendría en el orden Populetalia albae. Además, en el caso de las dos primeras asociaciones comentadas, éstas junto con el resto de bosques riparios de aliso, fresno y abedul del ámbito cántabro-atlántico del SW europeo (N España, W Francia e Islas Británicas) se integrarían en una nueva alianza, Hyperico androsaemi-Alnion glutinosae. Dentro de ella, la subalianza Hyperico androsaemi-Alnenion glutinosae agruparía a las comunidades riparias de aliso presentes a lo largo de los territorios cantábricos y orocantábricos ibéricos.

Teniendo en cuenta las propuestas de unidades superiores realizadas por Mucina et al. (2016) y Biurrun et al. (2016), las comunidades vegetales a las que se alude en este trabajo se integrarían en el siguiente esquema sinaxonómico:

CI. SALICI PURPUREAE-POPULETEA NIGRAE (Rivas-Martinez \& Cantó ex Rivas-Martinez, Báscones, T.E. Diaz, FernándezGonzález \& Loidi 1991) Rivas-Martinez \& Cantó 2002

OR. ALNO-FRAXINETALIA EXCELSIORIDIS Passarge 1968

Al. Hyperico androsaemi-Alnion glutinosae (Amigo et al.1987) Biurrun, Campos, Garcia-Mijangos \& Loidi 2016

Subal. Hyperico androsaemi-Alnenion glutinosae Amigo et al. 1987

As. Hyperico androsaemi-Alnetum glutinosae (Br.-BI. 1967) Rivas-Martínez in Loidi 1983

variante típica (termófila no hiperoceánica)

subvariante típica

subvariante de Osmunda regalis

variante hiperoceánica de Woodwardia radicans

subvariante típica

subvariante de Osmunda regalis

As. Valeriano pyrenaicae-Alnetum glutinosae Amigo, J. Guitián \& F. Prieto 1987

subas. alnetosum glutinosae (tipica termo-mesotemplada inferior)

variante típica (termófila no hiperoceánica)

subvariante típica

subvariante meso-eutrofa

variante hiperoceánica de Woodwardia radicans

subvariante típica

subvariante meso-eutrofa

variante suboceánica de Fraxinus angustifolia y Salix salviifolia

subvariante típica

"alisedas orocantábricas occidentales no termófilas"

OR. Populion AlBAE Br.-BI. ex Tchou 1948

Al. Osmundo-Alnion glutinosae (Br.-Bl., P. Silva \& Rozeira 1956) Dierschke \& Rivas -Martínez in Rivas-Martínez 1975

Subal. Osmundo regalis-Alnenion glutinosae (Br.-Bl. et al. 1956) Dierschke \& Rivas-Martínez in Rivas-Martinez 1975

As. Senecioni bayonnensis-Alnetum glutinosae Amigo, J. Guitián \& F. Prieto 1987

variante tipica

subvariante tipica

subvariante mesotrofa de Fraxinus excelsior

facies tipica

facies de transición a alisedas de la Valeriano-Alnetum 


\section{Conclusiones}

La aportación de nueva información acerca de la composición florística de los bosques de ribera dominados por Alnus glutinosa existentes a lo largo de los territorios litorales y sublitorales del extremo NW de la Península Ibérica ha permitido mejorar el conocimiento de la distribución de las asociaciones vegetales previamente descritas en este ámbito geográfico, el fundamento de su diferenciación desde el punto de vista fitosociológico y su variabilidad florística interna.

Paralelamente, los resultados obtenidos ponen en evidencia la necesidad de profundizar en el conocimento de las características botánicas de otros bosques dominados por esta especie presentes en territorios más interiores, principalmente a lo largo de las cuencas media y alta del Río Miño, el tramo final del Río Sil, y las áreas de cabecera de los ríos Navia, Limia y Támoga.

Agradecimientos Los autores agradecen la ayuda prestada por Ramiro Alvite Díaz, Javier Amigo Vázquez, Alejandro Álvarez Hurtado, Luís Gómez-Orellana, Gabriel Lijó Pose, Hugo López Castro, Carlos Oreiro Rey, José Luís Penín Franco, Javier PereiraEspinel, Carlos Real Rodríguez y Manuel Rodríguez Romero durante la realización de los trabajos de campo. Parte de los inventarios inéditos aportados han sido obtenidos en el curso de los trabajos de campo realizados durante la vigencia del Convenio 2015-CP098, firmado entre la Confederación Hidrográficas Miño-Sil y Limia y la Universidad de Santiago de Compostela.

\section{Referencias bibliográficas}

Amigo, J. (1984). Estudio de los matorrales y bosques de la Sierra de Caurel (Lugo). Tesis Doctoral inédita. Facultad de Farmacia. Universidade de Santiago de Compostela.

Amigo, J. (2005). Las saucedas riparias de Salicion salviifoliae en Galicia (Noroeste de España). Lazaroa 26: 67-81.

Amigo, J., Guitián, J. \& Fernández Prieto, J.A. (1987). Datos sobre los bosques ribereños de aliso (Alnus glutinosa) cántabro-atlánticos ibéricos. V Jornadas de Fitosociología. Vegetación de riberas de agua dulce, II. Universidad de la Laguna. Sec. de Publicaciones. Ser. Informes, 22: 159176.

Amigo, J., Pulgar, I. \& Izco, J. (2009): Evidence of riverside ash tree forest in Southern Galicia (Northwestern Spain). Lazaroa 30: 181-189.

ANTHOS. Sistema de Información sobre plantas de España. http://www.anthos.es/

Bellot, F. (1951). Sinopsis de la vegetación de Galicia. Anal. Jard. Bot. Madrid X(17): 389-444.

Bellot, F. (1968). La vegetación de Galicia. Anales del Instituto A. J. Cavanilles XXIV: 3-306.

Biodiversidade ameazada

http://www.biodiversidade.eu/catalogo/mapa/
Biurrun, I., García-Mijangos, I. \& Loidi, J, (1994): Study of alder forests in the Basque Country and bordering territories by means of multivariate analysis. Bot. Helv. 104: $31-54$

Biurrun, I., Campos, J.A., Herrera, M., Loidi, J. \& GarcíaMijangos, I. (2014). A survey of the riverine forests of the northern Iberian Peninsula: numerical classification versus traditional syntaxonomy and relationships with climatic gradients. Documents phytosociologiques, Série 3, Vol. 1: 92-109.

Biurrun, I., Campos, J.A., García-Mijangos,I., Herrera, M. \& Loidi, J. (2016). Floodplain forests of the Iberian Peninsula: Vegetation classification and climatic features. Applied Vegetation Science 19: 336-354.

Braun-Blanquet, J., Pinto da Silva, A.R. \& Rozeira, A. (1956): Résultats de deux excursions géobotaniques à travers le Portugal septentrional et moyen. II. Chênaies à feuilles caduques (Quercion occidentale) et chênaies à feuilles persistantes (Quercion fagineae) au Portugal. Agronomia Lusitana 3(18): 167-234.

Braun-Blanquet, J.; 1967; Vegetationsskizzen aus dem Baskenland mit Ausblicken auf das weitere IberoAtlantikum. II Teil.; Vegetatio 141-4: 1-126.

Braun-Blanquet, J. (1979). Fitosociología. Bases para el estudio de las comunidades vegetales. Ed. Blume. Barcelona.

Casaseca, B. (1959). La vegetación y flora del término municipal de Santiago de Compostela Boletín de la Universidad Compostelana 67:297-349.

Castroviejo, S. (Coord.)(1986-2013). Flora Iberica. Vols. IVIII, X, XIV y XXI. Real Jardín Botánico. C.S.I.C. Madrid.

Catalán, P. (1987). Geobotánica de las cuencas BidasoaUrumea (NO de Navarra-NE de Guipúzcoa). Estudio ecológico de los suelos y de la vegetación de la cuenca de Artikutza (Navarra). Tesis doctoral ined. Universidad del País Vasco.

Commision of the European Communities (1991). CORINE Biotopes Manual. Habitats of the European Community. EUR 12587/3. Directorate General Environment, Nuclear Safety and Civil Protection. Office for official Publications of the European Communities. Luxembourg.

Chytrý, M. \& Otýpková, Z. (2003). Plot sizes used for phytosociological sampling of European vegetation. Journal of Vegetation Science 14: 563-570.

Dalda González J. 1972. Vegetación de la cuenca del Río Deo. Cuenca alta del Mandeo. Santiago de Compostela. Monografías de la Universidad de Santiago de Compostela 14.

Davies, C. E. \& Moss, D. (2002). EUNIS Habitat Classification. 2001 Work Programme. Final Report. 108 pp. European Environment Agency. Huntingdon. 
Davies, C.E., Moss, D. \& Hill, M.O. (2004). EUNIS Habitat Classification Revised 2004. Report to the European Topic Centre on Nature Protection and Biodiversity. European Environment Agency.

Dengler, J., Lobel, S. \& Dolnik, C. (2009). Species constancy depends on plot size-a problem for vegetation classification and how it can be solved. Journal of Vegetation Science 20: 754-766.

Devesa, J.A., Quintanar, A. \& García, M.Á. (2015): Flora Iberica. Vol. XVI(I). Real Jardín Botánico. C.S.I.C. Madrid.

Devillers, P. \& Devillers-Terschuren, J. (1996). A Classification of Palaeartic Habitats. Nature and environment, $\mathrm{n}^{\circ}$ 78. Council of Europe Publishing. Strasbourg.

Díaz González, T.E. (1975). La vegetación del litoral occidental asturiano. Revista de la Facultad de Ciencias de Oviedo 15(2)-16: 369-545.

Díaz González, T.E. \& Fernández Prieto J.A. (1994). La vegetación de Asturias. Itinera Geobotanica 8: 243-528.

Díaz González T.E., Andrés, J., Llamas, F., Herrero, L., \& Fernández, M.D. (1986). Datos sobre la vegetación de las olmedas y alisedas de la provincia de León (NW de España). Secr. Publ. Univ. La Laguna. Ser. Informes 22: 177-198.

Douda, J., Boublík, K., Slezak, M., Biurrun, I., Nociar, J., Alena Havrdová, A., Doudová, J., Aćić, S., Brisse, H., Brunet, J., Chytry, M., Claessens, H., Csiky, J., Didukh, Y., Dimopoulos, P., Dullinger, S., Una FitzPatrick, U., Guisan, A., Horchler, P.J., Hrivnák, R., Jandt, U., Kącki, Z., Kevey, B., Landucci, F., Lecomte, H., Lenoir, J., Paal, J., Paternoster,D., Pauli, H., Pielech, R., Rodwell, J.S., Roelandt, B., Svenning, J-C., Šibík, J., Šilc, U., Škvorc, Ž., Tsiripidis, I., Tzonev, R.T., Wohlgemuth, Th. \& Zimmermann, N.E. (2016). Vegetation classification and biogeography of European floodplain forests and alder cars. Applied Vegetation Science 19: 147-163.

EEA (2017): EUNIS habitat classification 2007 (Revised descriptions 2012). Accesible en: http://eunis.eea.europa.eu/habitats/4271

Fagúndez, J. (2003). Algunas citas florísticas gallegas. Nova Acta Cient. Comp. (Biol.) 13: 103-105.

Fagúndez, J. (2011). Catálogo de la flora vascular del Concello de Ferrol (A Coruña). Monografías de Botánica Ibérica $\mathrm{n}^{\circ} 10.151 \mathrm{pp}$.

Fernández Prieto, J.A. Amigo, J. \& Guitián, J. (1987). Sobre la presencia de Salix salviifolia Brot. y Fraxinus angustifolia Vahl en la cuenca del Río Navia (Galicia-Asturias). Anales del Jardín Botánico de Madrid 44(1): 109-112.

García-Mijangos, I., Biurrun, I., Darquistade, A., Herrera, M. \& Loidi, J. (2004). Nueva cartografía de los hábitats en los lugares de interés comunitario (L.I.C.) fluviales de Navarra. Manual de interpretación de los hábitats. Informe para Viveros y Repoblaciones de Navarra.
Gillet, F. (2000). La phytosociologie synusiale intégrée. Guide méthodologique. Documents du Laboratoire d'ecologie végétale. Université de Neuchâtel-Institut de Botanique. Neuchâtel. 68 pp.

Géhu, J.-M. (1998). Epistémologie de la typologie phytosociologique de la végétation. Itinera Geobotanica 11: $65-83$.

Guitián, J. (1984). Estudio de la vegetación herbácea de la Sierra del Caurel (Lugo). Tesis doctoral inédita. Facultad de Farmacia. Universidad de Santiago de Compostela.

Hansen E.M. \& Sutton, W. (2000). Phytophthora diseases of forest trees. 152 pp. Forest Research Laboratory, Oregon State University. Oregon. USA.

Haque, M.M. \& Díez, J.J. (2012). Susceptibility of common alder (Alnus glutinosa) seeds and seedlings to Phytophthora alni and other Phytophthora species. Forest Systems 21(2): 313-322.

Honrado, J., Alves, P., Nepomuceno Alves, H. \& Barreto Caldas, F. (2002): Notas do Herbário da Estaçao Florestal Nacional (LISFA): Fasc. XVI. XXXIII: Ten new syntaxa from the Miniensean biogeographic subsector (northestern Portugal). Silva Lusitana 10(2): 247-259.

Honrado, J., Alves, P., Lomba, A., Torres, T. \& Barreto Caldas, F. (2007): Ecology, diversity and conservation of relict laurel-leaved mesophytic scrublands in mainland Portugal, Acta Bot. Gallica 154(1): 63-77.

IGME (1982). Mapa Geológico de España. Escala 1:200.000. Hoja 8 (2-2): Lugo. Servicio de Publicaciones. Ministerio de Industria y Energía. Madrid.

IGME (1984). Mapa Geológico de España. Escala 1:200.000. Hoja 1 (2-1): La Coruña. Servicio de Publicaciones. Ministerio de Industria y Energía. Madrid.

Izco, J. (1987). Galicia. En: S. Rivas Martínez \& M. Peinado Lorca (Eds.): La Vegetación de España. Publicaciones de la Universidad de Alcalá de Henares. Madrid.

Izco, J. (1989). El Río Miño: barrera y camino en la migración de las plantas. Conferencias sobre el Río Miño: 87-97. Caixa Ourense. Ourense.

Izco, J., Rodríguez-Dacal, C. \& Sánchez, J.M. (1994): Análisis geobotánico de las caldas de Lugo. Estudios sobre el Balneario de Lugo. Memorias de la Real Academia de Farmacia 20: 75-103. Madrid.

Izco, J., Amigo, J. \& García-San León, D. (1999). Análisis y clasificación de la vegetación leñosa de Galicia (España). Lazaroa 20: 29-47.

Laínz, M. (1966). Aportaciones al conocimiento de la flora gallega, IV. Anales Inst. Forest. Invest. 10: 299-332.

Lara, F., Garilleti, R. \& Calleja, J.A. (2004). La vegetación de ribera de la mitad norte española. Monografías CEDEX M81. Ministerio de Fomento-Ministerio de Medio Ambiente. Madrid. 536 pp. 
López Castro, H., Rodríguez Guitián, M.A., Ramil Rego, P., Real, C. \& Ferreiro da Costa, J. (2015). A Fraga de Santo Estevo do Ermo (Barreiros, Lugo): un lugar clave para a conservación de pteridófitas no Norte de Galicia (NW España) Recursos Rurais $11: 37-50$.

Macías Vázquez, F. \& Calvo de Anta, R. (2001). Los suelos. En A. Precedo Ledo (Coord.): Atlas de Galicia. I. O Medio: 173-217. Sociedade para o Desenvolvemento Comarcal de Galicia. Consellería da Presidencia. Xunta de Galicia. Santiago de Compostela.

Mayor López, M. \& Fernández Benito, M. (2007). Flora y vegetación de Asturias. Aspectos ecológicos, geográficos y fitosociológicos. Cuadernos de campo de la Zona Occidental. Editorial CEP. S.L. Humanes de Madrid. Madrid. $270 \mathrm{pp}$.

Moreno Sáez, JC. \& Sainz Ollero, H. (1992). Atlas corológico de las monocotiledóneas endémicas de la Península Ibérica y Baleares Colección Técnica. ICONA. Ministerio de Agricultura, Pesca y Alimentación. Madrid. $354 \mathrm{pp}$.

Muñoz, F., Navarro, C. Quintanar, A. \& Buira, A. (2015). Flora iberica. Volumen IX. Real Jardín Botánico. C.S.I.C. Madrid.

Mucina, L., Bültmann, H., Dierßen, K., Theurillat, J.-P., Raus, Th., Čarni, A., Šumberová, K., Willner, W., Dengler, J., Gavilán García, R., Chytrý, M., Hajek, M., Di Pietro, R., lakushenko, D., Pallas, J., Daniëls, F.J.A., Bergmeier, E., Santos Guerra, A., Ermakov, N., Valachovič, M., Schaminée, J.H.J., Lysenko, T., Didukh, Y.P., Pignatti, S., Rodwell, J.S., Capelo, J., Weber, H.E., Solomeshch, A., Dimopoulos, P., Aguiar, C., Hennekens, S.M., \& Tichý, L. (2016). Vegetation of Europe: hierarchical floristic classification system of vascular plant, bryophyte, lichen, and algal communities. App. Veg. Sci. 19 (Suppl. 1): 3-264.

Mueller-Dombois, D. \& Ellenberg, H. (2002). Aims and methods of vegetation ecology. The Blackburn Press. Caldwell, New Jersey, USA. 547 pp.

Onaindia, M., García, I. \& Herrera, M. (1987). Vegetación riparia en la provincia de Vizcaya. Publicaciones de la Universidad de La Laguna. Serie Informes 22: 267-271.

Pino Pérez, R., Silva-Pando, F.J., Camaño Portela, J.L., Pino Pérez, J.J., García Martínez, X.R. \& Gómez Vigide, F. (2009). Atlas y catálogo de la familia Amaryllidaceae de Galicia. Boletín BIGA 6: 83-107.

Pulgar Sañudo, I. (1999). La vegetación de la Baixa Limia y sierras del entorno. Tese de Doutoramento inédita. Facultade de Bioloxía. USC. Santiago de Compostela. 275 pp.

Ramil-Rego, P., Ferreiro da Costa, J. (2016): Biodiversidad del corredor fluvial del río Miño: tramo Ponte OmbreiroCaneiro do Anguieiro (Lugo). En: J.M. Crecente Maseda \& S. González Soutelo (Eds. científicos): Dos mil años del balneario de Lugo. Un modelo de activación del patrimonio termal: 64-96. Lugo.
Ramil-Rego, P., de Nóvoa Fernández, B., Ferreiro da Costa, J., Hinojo Sánchez, B.A. \& Rubinos Román, M.A. (2010). Medio natural e rede fluvial. En: A. Meixide Vecino, $M$. Fernández Fernández \& M.F. Marey Pérez (Coords.): Plan estratéxico da Provincia de Lugo: 604-638. USCFundación Caixa Galicia-Deputación de Lugo. Lugo.

Rivas-Martínez, S., Fernández-González, F., Loidi, J., Lousâ, M. \& Penas, A. (2001): Syntaxonomical checklist of vascular plant communities of Spain and Portugal to association level. Itinera Geobotanica 14, 5-341.

Rivas-Martínez, S., Díaz, T.E., Fernández-González, F., Izco, J., Loidi, J., Lousâ, M. \& Penas, A. (2002). Addenda to the syntaxonomical checklist of 2001 . Itinera Geobotanica 15, 2 vol.

Rodríguez González, P.M. (2008): Os bosques higrófilos Ibero-atlânticos. Tese de Doutoramento. 245 pp. Instituto Superior de Agronomia. Lisboa.

Rodríguez Guitián, M.A. (2004). Aplicación de criterios botánicos para a proposta de medidas de xestión sustentable das masas arborizadas autóctonas do Subsector Galaico-Asturiano setentrional. Tesis doctoral inédita. Escola Politécnica Superior de Lugo. USC. Lugo. $620 \mathrm{pp}$.

Rodríguez Guitián, M.A. (2005). Avaliación da diversidade sílvica do subsector galaico-asturiano septentrional: tipos de bosques, valor para a conservación e principais ameazas. Recursos Rurais, Serie Cursos 2: 23-44.

Rodríguez Guitián, M.A. (2006). Acerca de la identidad fitosociológica de los hayedos silicícolas sublitorales del centro de la cornisa cantábrica. Lazaroa 27: 59-78.

Rodríguez Guitián, M.A. (2010a). Temperate riverside forests without alder trees at the NW of the Iberian Peninsula: ecology, phytosociological profile and interest for preservation policies. Lazaroa 31: 9-37.

Rodríguez Guitián, M.A. (2010b). Aportacións sobre a tipoloxía e composición florística dos bosques mesófilos de quercíneas do occidente da Cornixa Cantábrica (NW Ibérico). Recursos Rurais 2(6): 35-73.

Rodríguez Guitián, M.A. \& Ramil Rego. P. (2007). Revisión de las clasificaciones climáticas aplicadas al territorio gallego desde una perspectiva biogeográfica. Recursos Rurais 1(3): 31-53.

Rodríguez Guitián, M.A. \& Ramil-Rego, P. (2008). Fitogeografía de Galicia (NW Ibérico): análisis histórico y nueva propuesta corológica. Recursos Rurais 1(4): 19-50.

Rodríguez Guitián, M.A., Amigo Vázquez, J. \& Romero Franco, R. (2000). Aportaciones sobre la interpretación, ecología y distribución de los bosques supratemplados naviano-ancarenses. Lazaroa 21: 51-71. 
Rodríguez-Guitián, M.A., Romero Franco, R., Díaz Varela, R.A. \& Ferreiro da Costa, J. (2014): Tipoloxía e valor de conservación dos bosques da Reserva da Biosfera "Os Ancares Lucenses e Montes de Cervantes, Navia e Becerreá" (Lugo, Galicia, España). En: A. Rigueiro Rodríguez \& M.A. Rodríguez Guitián (Coords.): Ciclo de Conferencias Luís Asorey 2014: A Reserva da Biosfera dos Ancares Lucenses", Recursos Rurais. Serie Cursos: 53-111.

Rodríguez Guitián, M.A., Real, C., Romero Franco, R. \& Álvarez-Hurtado, A. (2017). Phytosociological framework and conservation value of supratemperate riparian birch forest of the NW Iberian Peninsula. Lazaroa 38(2): 87-125.

Rodwell, J.S. (Ed.)(1991). British Plant Communities. Vol. I. Woodlands and scrub. Cambrigde University Press. Cambrigde. 395 pp.

Romero Buján, M.I. (1993). La vegetación del valle del río Cabe (Terra de Lemos, Lugo). Tesis Doctoral (inédita). Facultade de Bioloxía. USC. Santiago de Compostela.

Silva-Pando, F. J. (1990): La Flora y vegetación de la Sierra de Ancares: base para la planificación y ordenación forestal. Mem. Doc. Inéd. Facultad de Ciencias Biológicas. Universidad Complutense. Madrid. 532 pp.
Sitte, P., Weiler, E.W., Kadereit, J.W., Bresinsky, A. \& Körner, C. (2004). Strasburger. Tratado de Botánica. $35^{\mathrm{a}}$ ed. Ed. Omega. Barcelona. 1.134 pp.

SIVIM. Sistema de Información de la vegetación ibérica y macaronésica. http://www.sivim.info/sivi/

Talavera, S., Andrés, C., Arista, M., Fernández Piedra, M.P., Rico, E., Crespo, M.B., Quintanar, A., Herrero, A. \& Aedo, C. (2014): Flora iberica. Vol. XX. Real Jardín Botánico. C.S.I.C. Madrid.

Tutin, T.G., Heywood, V.H., Burges, N.A., Moore, D.M., Valentine, D.H., Walters, S.M. \& Webb, D.A. (Eds.) (19641980). Flora Europaea, Vols. 1-5. Cambrigde University Press. Cambrigde.

Valcárcel, V. \& Vargas, P. (2002). Hacia un tratamiento taxonómico de las hiedras (Hedera L., Araliaceae) ibéricas: de caracteres morfológicos a moleculares. Anales del Jardín Botánico de Madrid, 59(2): 363-368. 


\section{ANEXO}

Tablas de inventarios florísticos inéditos de las comunidades de alisedas riparias estudiadas

Tabla la. Alisedas galaico-portuguesas (Senecioni bayonnensis-Alnetum glutinosae) variante típica, subvariante típica, facies típica

\begin{tabular}{|c|c|c|c|c|c|c|c|c|c|c|c|c|c|c|c|c|c|c|c|}
\hline $\mathrm{N}^{0}$ de orden & 1 & 2 & 3 & 4 & 5 & 6 & 7 & 8 & 9 & 10 & 11 & 12 & 13 & 14 & 15 & 16 & 17 & 18 & 19 \\
\hline Altitud (m) & 445 & 350 & 535 & 240 & 530 & 525 & 433 & 500 & 585 & 445 & 490 & 485 & 495 & 362 & 295 & 345 & 510 & 365 & 255 \\
\hline Pendiente $\left({ }^{\circ}\right)$ & $<2$ & 6 & $<2$ & 2 & $<2$ & 2 & 6 & $<2$ & 6 & $<2$ & $<2$ & $<2$ & $<2$ & 2 & 2 & SSW & $<2$ & 2 & 4 \\
\hline Orientación & SW & $\mathrm{N}$ & NW & ESE & ESE & NE & $S$ & WSW & $S$ & NW & $\mathrm{N}$ & W & SSW & W & $S$ & $<2$ & W & SW & WSW \\
\hline Altura de copas (m) & 14 & 10 & 20 & 18 & 20 & 16 & 12 & 16 & 18 & 16 & 20 & 14 & 16 & 16 & 14 & 16 & 16 & 12 & 20 \\
\hline Cob. $E_{1}(\%)$ & 100 & 95 & 100 & 100 & 100 & 100 & 95 & 100 & 100 & 90 & 85 & 100 & 100 & 90 & 95 & 100 & 100 & 100 & 90 \\
\hline Cob. $E_{2}(\%)$ & 20 & 15 & 15 & 25 & 20 & 15 & 15 & 25 & 15 & 15 & 5 & 20 & 20 & 15 & 25 & 20 & 20 & 60 & 25 \\
\hline Cob. $E_{3}(\%)$ & 100 & 85 & 100 & 90 & 100 & 55 & 85 & 100 & 90 & 95 & 100 & 100 & 95 & 95 & 95 & 95 & 100 & 100 & 90 \\
\hline Área $\left(\mathrm{m}^{2}\right)$ & 300 & 200 & 120 & 200 & 200 & 300 & 300 & 240 & 500 & 300 & 300 & 400 & 240 & 300 & 500 & 300 & 300 & 300 & 600 \\
\hline $\mathrm{N}^{0}$ de taxones & 37 & 43 & 33 & 41 & 33 & 34 & 34 & 31 & 46 & 46 & 36 & 56 & 39 & 40 & 46 & 47 & 55 & 45 & 61 \\
\hline \multicolumn{20}{|l|}{$\overline{E_{1}(>4,0 \mathrm{~m})+E_{2}(>1,5-4,0 \mathrm{~m}):}$} \\
\hline Alnus glutinosa & 5 & 3 & 5 & 5 & 5 & 5 & 4 & 5 & 4 & 4 & 4 & 4 & 4 & 5 & 4 & 4 & 4 & 5 & 5 \\
\hline Salix atrocinerea & 1 & 3 & 1 & 1 & 1 & 1 & 2 & 1 & 1 & 2 & 1 & 1 & 2 & 1 & 2 & 1 & 2 & 1 & . \\
\hline Quercus robur & 1 & + & 1 & 1 & . & 1 & 2 & . & 2 & 1 & 1 & 1 & 1 & + & 2 & 2 & + & + & 1 \\
\hline Frangula alnus & . & . & 1 & . & 1 & 1 & . & . & 1 & 1 & 1 & + & 2 & 1 & . & 1 & 1 & + & 1 \\
\hline Sambucus nigra & 1 & 1 & 1 & 1 & 1 & 1 & . & . & 1 & + & . & 1 & . & . & 1 & 1 & . & 2 & . \\
\hline Laurus nobilis & 1 & . & . & 1 & . & . & . & 1 & r & . & . & . & . & 1 & . & . & . & 1 & . \\
\hline Castanea sativa & 1 & . & . & . & . & . & . & . & . & . & . & . & + & 1 & . & . & . & . & 1 \\
\hline \multicolumn{20}{|l|}{$\begin{array}{l}\mathrm{E}_{3}(<1,5 \mathrm{~m}) \\
\text { Taxones dife }\end{array}$} \\
\hline Narcissus cyclamineus & . & 1 & 1 & + & + & 1 & 2 & 3 & 1 & 2 & 1 & + & 2 & 1 & . & . & . & + & . \\
\hline Fraxinus angustifolia & . & . & . & . & . & . & . & . & . & . & . & . & . & . & . & . & . & + & 1 \\
\hline Echium Iusitanicum & . & . & . & . & . & . & . & . & + & . & . & . & . & . & . & . & . & . & . \\
\hline \multicolumn{20}{|c|}{ Taxones diferenciales frente a Hyperico-Alnetum } \\
\hline Deschampsia subtriflora & 1 & + & 1 & + & 1 & 1 & 2 & + & + & + & $\cdot$ & + & + & + & 1 & + & + & + & $\cdot$ \\
\hline Betula pubescens & 1 & . & 1 & 1 & 1 & 1 & 1 & 1 & 1 & 1 & 2 & 1 & 3 & 1 & . & 2 & 2 & 1 & . \\
\hline Viola palustris & 1 & 1 & 1 & 2 & 1 & . & . & 1 & 1 & . & . & . & 1 & 1 & 2 & . & 1 & 2 & . \\
\hline Osmunda regalis & . & + & . & + & + & . & 2 & . & . & 1 & + & + & . & 1 & + & . & + & . & 1 \\
\hline Omphalodes nitida & 1 & 1 & . & + & 1 & . & . & . & + & + & . & + & 1 & 1 & . & 1 & . & . & + \\
\hline Erica arborea & + & + & . & + & + & + & 1 & . & + & + & . & . & 1 & 1 & . & . & . & . & + \\
\hline Angelica major & + & + & $r$ & + & + & . & + & + & . & . & . & . & + & . & . & 1 & . & + & . \\
\hline Caltha palustris & 1 & 1 & . & . & . & . & . & 1 & . & . & + & . & + & . & + & + & . & + & . \\
\hline Carex laevigata & + & . & . & . & . & + & . & . & . & . & + & . & + & . & + & . & + & . & + \\
\hline Pyrus cordata & + & . & . & . & . & . & . & . & . & 1 & 1 & . & . & . & . & 1 & . & . & . \\
\hline Saxifraga spathularis & . & . & . & . & . & . & . & . & 1 & . & . & . & . & 1 & . & . & . & . & . \\
\hline Narcissus asturiensis & . & . & . & . & . & . & . & . & . & . & . & + & . & . & . & 1 & . & . & . \\
\hline Aquilegia dichroa & . & . & . & . & . & . & . & . & . & . & . & . & . & . & . & . & . & . & + \\
\hline Physospermum cornubiense & . & . & . & . & . & . & . & . & . & . & . & . & . & . & . & . & . & . & + \\
\hline Solidago virgaurea & . & . & . & . & . & . & . & . & . & . & . & . & . & . & . & . & . & . & + \\
\hline Narcissus triandrus & . & . & . & . & . & . & . & . & . & . & . & . & . & . & . & . & . & . & + \\
\hline
\end{tabular}

\begin{tabular}{|c|c|c|c|c|c|c|c|c|c|c|c|c|c|c|c|c|c|c|c|}
\hline $\begin{array}{l}\text { Taxones característicos } \\
\text { Lonicera hispanica }\end{array}$ & $\frac{1}{2}$ & 2 & + & 2 & 1 & 2 & 2 & 1 & 1 & 1 & 1 & 1 & 1 & 1 & 1 & 2 & 1 & 2 & 1 \\
\hline Dryopteris affinis & 2 & + & 2 & 1 & 2 & 2 & 1 & 1 & 1 & 1 & 1 & 1 & + & 2 & 1 & 2 & 1 & 1 & 1 \\
\hline Athyrium filix-femina & 2 & 2 & 2 & 2 & 2 & $\cdot$ & 2 & 1 & 1 & 2 & 3 & 2 & + & 2 & 2 & 1 & 1 & 3 & 1 \\
\hline Hedera hibernica & 2 & . & 1 & 4 & 1 & 1 & 2 & 2 & 1 & 1 & 2 & . & 1 & 2 & 2 & 2 & 1 & 3 & 2 \\
\hline Euphorbia dulcis & 2 & 1 & . & + & + & 1 & 1 & + & + & 1 & 1 & . & 1 & 2 & 1 & 2 & 2 & + & 1 \\
\hline Blechnum spicant & 2 & 1 & 2 & + & 1 & + & 1 & 1 & 1 & 1 & + & + & 1 & 1 & 1 & 1 & + & · & . \\
\hline Viola riviniana & . & 1 & + & + & + & $\cdot$ & + & + & + & . & . & + & + & 1 & 1 & + & 2 & + & + \\
\hline Ranunculus ficaria & . & 2 & . & . & $\cdot$ & + & . & 1 & 1 & + & 1 & 1 & 1 & 1 & 2 & 2 & 2 & 1 & 1 \\
\hline Primula acaulis & · & + & · & . & · & + & 1 & + & 1 & + & + & 1 & · & · & · & + & 1 & · & + \\
\hline Senecio nemorensis & 1 & . & · & 1 & 1 & ' & - & + & . & . & . & 1 & 1 & 1 & 2 & 2 & . & 2 & . \\
\hline Carex reuteriana & · & . & · & 1 & · & · & · & · & 1 & 1 & · & + & 1 & + & · & · & $\cdot$ & · & 2 \\
\hline Solanum dulcamara & · & . & + & + & · & · & · & · & . & · & · & 1 & · & . & · & · & + & · & · \\
\hline Myosotis martini & · & . & · & . & + & · & · & · & . & · & · & · & · & . & 1 & 1 & · & · & · \\
\hline Polystichum setiferum & · & . & · & · & · & + & · & · & . & · & · & · & · & . & · & · & · & · & 2 \\
\hline Carex remota & · & . & + & · & · & · & $\cdot$ & · & · & · & · & $\cdot$ & · & . & · & · & · & · & 1 \\
\hline Brachypodium sylvaticum & . & . & · & . & . & . & . & · & - & . & . & . & · & . & + & . & . & . & + \\
\hline Carex pendula & · & . & + & · & · & · & · & · & · & · & · & $\cdot$ & . & $r$ & . & . & . & $\cdot$ & . \\
\hline
\end{tabular}




\begin{tabular}{|c|c|c|c|c|c|c|c|c|c|c|c|c|c|c|c|c|c|c|c|}
\hline $\mathrm{N}^{0}$ de orden & 1 & 2 & 3 & 4 & 5 & 6 & 7 & 8 & 9 & 10 & 11 & 12 & 13 & 14 & 15 & 16 & 17 & 18 & 19 \\
\hline \multicolumn{20}{|c|}{ Taxones característicos de Quercetea robori-petraeae y Carpino-Fagetea } \\
\hline Holcus mollis & 3 & + & 2 & 1 & 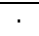 & 1 & 2 & 1 & + & 2 & 1 & . & 1 & 2 & 1 & + & 1 & 2 & 1 \\
\hline Polypodium vulgare & + & + & . & 1 & + & . & 1 & + & 1 & + & + & + & + & 1 & 1 & + & + & . & 1 \\
\hline Teucrium scorodonia & 1 & 1 & . & 1 & 2 & . & 1 & . & 1 & 1 & 1 & 1 & + & 1 & + & + & 2 & 1 & 1 \\
\hline Stellaria holostea & 1 & 1 & 1 & . & 1 & + & . & . & + & + & 1 & 1 & + & + & . & 1 & + & . & . \\
\hline Dryopteris dilatata & 1 & 1 & 2 & + & 1 & 1 & . & 2 & . & 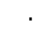 & . & . & . & 1 & + & + & . & . & . \\
\hline Potentilla sterilis & . & . & . & $\cdot$ & $\cdot$ & · & . & · & . & + & + & 1 & + & r & + & $\cdot$ & $\cdot$ & . & $\cdot$ \\
\hline Hyacinthoides non-scripta & + & . & . & $\cdot$ & $\cdot$ & . & . & . & + & + & . & + & $\cdot$ & $\cdot$ & $\cdot$ & $\cdot$ & . & . & . \\
\hline Crepis lampsanoides & . & . & . & $\cdot$ & $\cdot$ & + & . & . & 1 & $\cdot$ & $\cdot$ & $\cdot$ & + & $\cdot$ & $\cdot$ & $\cdot$ & $\cdot$ & $\cdot$ & 1 \\
\hline Ajuga reptans & . & 1 & . & . & . & . & . & . & . & . & . & . & . & . & 1 & 1 & $\cdot$ & 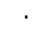 & + \\
\hline
\end{tabular}

\begin{tabular}{|c|c|c|c|c|c|c|c|c|c|c|c|c|c|c|c|c|c|c|c|}
\hline \multicolumn{20}{|l|}{ Otros taxones } \\
\hline Rubus sp. & 1 & 1 & 1 & 1 & 2 & . & 2 & 2 & 1 & 1 & + & + & 1 & 2 & 1 & 1 & 1 & 3 & 1 \\
\hline Oenanthe crocata & 1 & 1 & + & 2 & + & 1 & 1 & 1 & + & 1 & + & 1 & 1 & + & . & + & + & 2 & + \\
\hline Brachypodium rupestre & + & + & + & 1 & 1 & . & 2 & . & 2 & 3 & 4 & 4 & 3 & 3 & 3 & 1 & 2 & 2 & 3 \\
\hline Dactylis glomerata & . & 1 & 1 & . & . & + & 1 & . & . & 1 & 1 & 2 & 1 & . & + & + & 1 & 1 & 1 \\
\hline Pteridum aquilinum & + & + & $r$ & . & . & 1 & 1 & . & . & 1 & 1 & + & 2 & + & 1 & $\cdot$ & $\cdot$ & 1 & + \\
\hline Ranunculus repens & . & + & . & + & . & + & . & . & + & + & + & 1 & + & . & + & . & + & + & + \\
\hline Cardamine pratensis & . & + & . & . & + & . & + & 1 & . & . & . & + & 1 & . & 1 & 1 & 1 & + & . \\
\hline Umbilicus rupestris & . & + & . & . & . & . & + & + & + & + & + & + & . & . & . & + & + & ${ }^{\prime}$ & + \\
\hline Heracleum sphondylium & . & + & . & . & . & 1 & . & + & 1 & 1 & & + & . & . & . & 1 & 1 & ${ }^{\prime}$ & + \\
\hline Geranium robertianum & . & + & + & . & . & + & . & + & . & + & . & 1 & . & . & + & 1 & + & ${ }^{\prime}$ & . \\
\hline Rumex acetosa & . & + & . & + & . & . & + & . & . & + & . & . & . & . & + & . & 1 & + & + \\
\hline Scrophularia auriculata & . & . & + & + & + & . & $\cdot$ & . & . & + & + & + & . & . & + & $\cdot$ & + & . & . \\
\hline Narcissus bulbocodium & + & $\cdot$ & $\cdot$ & . & . & $\cdot$ & + & $\cdot$ & + & + & $\cdot$ & $\cdot$ & . & . & + & $\cdot$ & + & $\cdot$ & . \\
\hline Peucedanum lancifolium & . & . & . & + & . & . & + & . & . & . & . & . & + & . & . & . & + & + & 1 \\
\hline Senecio aquaticus & 1 & + & . & . & . & . & . & . & . & + & . & . & . & . & . & + & . & + & . \\
\hline Agrostis capillaris & . & . & 1 & . & . & . & + & . & . & . & 1 & . & . & . & . & $\cdot$ & 1 & 1 & . \\
\hline Digitalis purpurea & + & . & + & . & . & . & . & . & + & . & & . & . & . & + & . & . & . & + \\
\hline Prunella vulgaris & . & + & $\cdot$ & + & . & · & . & . & + & . & . & · & . & . & + & $\cdot$ & $\cdot$ & 1 & . \\
\hline Lamium maculatum & . & + & $\cdot$ & + & . & . & $\cdot$ & . & . & + & + & . & . & . & 1 & $\cdot$ & $\cdot$ & $\cdot$ & . \\
\hline Anthoxanthum amarum & . & . & $\cdot$ & . & + & · & $\cdot$ & . & $\cdot$ & · & 1 & 2 & . & $\cdot$ & $\cdot$ & $\cdot$ & $\cdot$ & $\cdot$ & + \\
\hline Arrhenatherum bulbosum & - & - & . & . & + & · & $\cdot$ & . & . & . & . & . & . & 1 & . & $\cdot$ & 2 & 1 & . \\
\hline Centaurea rivularis & . & + & . & . & . & - & + & . & . & . & . & . & . & $r$ & . & . & + & . & . \\
\hline Galium aparine & . & . & . & . & . & + & . & . & . & . & . & + & . & . & . & + & + & . & . \\
\hline Cardamine hirsuta & . & . & . & . & . & . & $r$ & . & . & . & & 1 & . & . & . & . & + & . & + \\
\hline Pentaglottis sempervirens & . & . & . & . & $r$ & . & . & . & . & + & & 1 & . & . & . & . & + & . & \\
\hline
\end{tabular}

Taxones presentes en menos de cuatro inventarios:

$\mathbf{E}_{\mathbf{1}}+\mathrm{E}_{\mathbf{2}}$ : Corylus avellana: 2 en 8, + en 9 e 1 en 19; Crataegus monogyna: 2 en 19; Cytisus scoparius: + en 9 y + en 19; Cytisus striatus: + en 9; llex aquifolium: + en 15; Rosa gr. canina: + en 10 y + en 19. Taxones característicos de Quercetea robori-petraeae y CarpinoFagetea: Anemone nemorosa: + en 19; Aquilegia vulgaris: 1 en 10; Conopodium majus: + en 9 y + en 17; Dryopteris filix-mas: 1 en 12 y + en 19; Euphorbia amygdaloides: 1 eb 10, 1 eb 15 y + en 19; Hypericum pulchrum: + en 18; Luzula sylvatica: 3 en 9 y 2 en 19; Oxalis acetosella: 1 en 8, + en 14 y 1 en 16; Stachys officinalis: 1 en 14 y + en 16. Otros taxones: Angelica sylvestris: 1 en 15; Anthoxanthum odoratum: + en 14; Antinoria agrostidea: + en 12; Apium nodiflorum: + en 18; Asphodelus lusitanicus: 1 en 16; Avenula sulcata: + en 19; Bryonia dioica: + en 12; Calystegia sepium: 1 en 18; Carex cariophyllea: r en 15; Carex echinata: e en 3; Carex lusitanica: + en 6, + en 12 y + en 17; Chelidonium majus: + en 12; Chrysosplenium oppositifolium: 2 en 8 y 1 en 16; Cirsium palustre: + en 6, + en 9 y + en 17; Crocus serotinus: 1 en 13 y + en 16; Cruciata glabra: + en 7 y + en 14; Epilobium hirsutum: + en 3; Epilobium sp.: + en 12 y + en 18; Eupatorium cannabinum: 1 en 11; Festuca gr. rubra: + en 2 y + en 19; Fragaria vesca: + en 17; Galeopsis tetrahit: + en 12; Galium palustre: 1 en 17 y + en 18; Geranium lucidum: r en 19; Geum urbanum: 1 en 15 y + en 19; Glechoma hederacea: + en 19; Glyceria fluitans: 1 en 17; Hieracium sp.: + en 19; Holcus lanatus: 1 en 12; Hypericum sp.: + en 4; Hypericum undulatum: + en 17; Juncus effusus: r en 2, r en 17 y + en 18; Juncus sp.: + en 12; Lapsana communis: + en 10 y + en 19; Limniris pseudacorus: + en 6 y + en 17; Linaria triornithophora: r en 19; Lotus pedunculatus: + en 17; Lycopus europaeus: 1 en 18; Malva sp.: + en 11; Molinia caerulea: r en 19; Peucedanum gallicum: + en 1; Plantago lanceolata: $r$ en 9; Poa annua: + en 12; Poa trivialis: + en 15; Polygonum hydropiper + en 18; Polygonum persicaria: + en 4; Rumex acetosella: + en 16; Rumex obtusifolius: + en 4 y + en 6; Ruscus aculeatus: 1 en 19; Scutellaria minor: + en 17; Silene dioica: 2 en 16; Sparganium neglectum: + en 12 y + en 17; Stellaria media: + en 12; Taraxacum gr. officinale: + en 12; Tritonia x crocosmiflora: + en 4 y + en 15; Urtica dioica: 1 en 1, + en 6 y 1 en 12; Valeriana dioica: + en 16; Valeriana officinalis: 1 en 17; Veronica chamaedrys: + en 12; Vitis sp.: + en 18.

\section{Procedencia de los inventarios:}

1: C: Mesía, Cabrui, Río da Tarroeira (565/4774); 2: C: Santa Catalina de Armada, entre Santa Comba y Xallas, Rego da Abelleira (516/4766); 3: Lu: Palas de Rei, carretera a Quindimil, Río Pequeño (592/4748); 4: C: Santiago de Compostela, Albudiña, A Ponte Vilar, Rego de Oufín (536/4755); 5: Lu: Palas de Rei, Ferreira de Negral, Río Ferreira (596/4756); 6: Lu: Antas de Ulla, entre Vilanuñe y A Caira, Rego do Ribeiro (593/4734); 7: C: Aranga, Bexo, Río de Bexo (574/4783); 8: C: Boimorto, Corneda, Rego de Corneda (575/4759); 9: Po: A Cañiza, Petán, entre A Aldeíña y Senande, Río Deva (558/4676); 10: Po: Lalín, Moimenta, Cancela, Río de Mouroces, aguas arriba de Ponte das Abellas (575/4731); 11: Lu: Antas de Ulla, Casa de Naia, Pontepedriña, margen izquierda do Río Ulla (594/4737); 12: Lu: Monterroso, entre Monterroso y Os Carreiros, Río Ulla (593/4738); 13: Lu: Palas de Rei, Río Ferreira, entre Berbetouros y Vilamaior de Negral (598/4753); 14: C: Irixoa, Verís, Río Zarzo aguas abajo de Ponte da Fraga (576/4791); 15: C: Zas, Limideiro, Brañas Grandes, 
Rego de Porto Carreiro (504/4762); 16: C: Mesía, Xanceda, aguas arriba del puente entre O Campo y Xestosa, Rego do Molar (562/4769); 17: Lu: Antas de Ulla, O Barreiro, A Ponte Paredes, Río Pequeno (589/4740); 18: C: Mesía, Albixoi, Maruzo, Río Maruzo (563/4766); 19: Ou: Cartelle, Outomuro, entre O Mundil y O Viso, margen derecha del Río Arnoia, aguas abajo de A Ponte Nova (578/4673).

Tabla Ib. Alisedas galaico-portuguesas (Senecioni bayonnensis-Alnetum glutinosae) variante típica, subvariante típica, facies típica (cont) 


\begin{tabular}{|c|c|c|c|c|c|c|c|c|c|c|c|c|c|c|c|c|c|c|}
\hline $\mathrm{N}^{0}$ de orden & 1 & 2 & 3 & 4 & 5 & 6 & 7 & 8 & 9 & 10 & 11 & 12 & 13 & 14 & 15 & 16 & 17 & 18 \\
\hline \multicolumn{19}{|c|}{ Taxones característicos de asociación y unidades superiores } \\
\hline Athyrium filix-femina & 3 & + & 2 & 3 & + & 1 & 2 & 2 & 2 & 1 & 1 & 2 & 2 & 4 & 3 & 1 & 1 & 1 \\
\hline Hedera hibernica & 1 & 1 & 2 & 1 & 1 & 2 & + & 1 & 1 & 1 & 2 & 2 & 1 & 1 & 1 & 1 & 2 & 2 \\
\hline Lonicera hispanica & . & 1 & 1 & 1 & + & 1 & 1 & + & 1 & . & 2 & 1 & 1 & 2 & + & 1 & 1 & . \\
\hline Dryopteris affinis & 1 & + & . & . & 2 & 1 & 1 & 1 & + & 1 & 1 & 1 & 1 & 2 & 1 & 1 & . & . \\
\hline Primula acaulis & . & 1 & 1 & + & 1 & 1 & 1 & 1 & $r$ & + & + & + & . & . & 1 & + & . & . \\
\hline Blechnum spicant & . & 1 & + & + & + & 1 & 1 & + & 1 & . & + & + & . & . & 1 & 1 & . & + \\
\hline Euphorbia dulcis & + & 1 & . & + & 1 & 2 & 2 & 2 & . & . & + & . & . & + & 1 & 1 & . & . \\
\hline Ranunculus ficaria & . & 1 & 1 & . & 1 & . & 1 & 1 & $\cdot$ & 1 & 2 & . & 1 & . & + & 1 & . & . \\
\hline Senecio nemorensis & . & + & 2 & + & . & . & . & 2 & + & . & . & + & . & . & + & + & . & . \\
\hline Polystichum setiferum & . & . & . & . & + & . & . & 1 & + & + & 1 & . & . & . & + & . & 1 & 1 \\
\hline Brachypodium sylvaticum & . & . & . & & 1 & . & . & . & . & 1 & + & + & + & . & . & . & + & . \\
\hline Hypericum androsaemum & . & . & . & . & + & + & . & . & + & . & . & + & . & . & . & + & . & . \\
\hline Carex remota & . & + & . & & . & . & . & . & . & 2 & 1 & . & . & . & . & . & . & 1 \\
\hline Carex pendula & . & . & . & & . & . & . & . & 1 & . & ${ }^{\circ}$ & + & . & . & . & . & + & + \\
\hline
\end{tabular}

Taxones característicos de Quercetea robori-petraeae y Carpino-Fagetea

\begin{tabular}{|c|c|c|c|c|c|c|c|c|c|c|c|c|c|c|c|c|c|c|}
\hline Teucrium scorodonia & + & 1 & 1 & 1 & + & 1 & + & 1 & 1 & . & 1 & + & 2 & 1 & + & + & . & \\
\hline Holcus mollis & 1 & 1 & 1 & 3 & . & & 1 & 2 & + & . & 1 & . & 1 & 3 & 1 & + & . & \\
\hline Crepis lampsanoides & 1 & 1 & . & . & 1 & + & 1 & 1 & 1 & + & 1 & + & + & $\cdot$ & . & + & . & \\
\hline Polypodium vulgare & + & + & + & . & + & + & + & + & + & . & + & . & + & . & . & + & . & 1 \\
\hline Luzula sylvatica & . & 2 & . & . & 1 & 4 & . & 1 & 1 & + & 2 & 1 & + & . & 1 & 3 & . & \\
\hline Ajuga reptans & + & . & . & . & + & 2 & . & 1 & + & . & . & . & + & 1 & 1 & + & . & \\
\hline Anemone nemorosa & + & + & . & + & 2 & & 2 & . & . & + & . & . & . & . & 1 & . & . & \\
\hline Stellaria holostea & 2 & . & 1 & . & 1 & 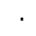 & + & . & . & . & + & . & 1 & + & . & . & . & \\
\hline Dryopteris dilatata & . & . & + & 1 & . & & . & 1 & + & . & . & + & 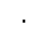 & 2 & + & . & . & \\
\hline Euphorbia amygdaloides & + & . & . & . & 1 & & . & . & $r$ & + & + & . & + & . & . & + & . & \\
\hline Hyacinthoides non-scripta & . & . & . & . & + & & 1 & . & . & ${ }^{\circ}$ & + & . & & & 2 & 1 & . & \\
\hline
\end{tabular}

\begin{tabular}{|c|c|c|c|c|c|c|c|c|c|c|c|c|c|c|c|c|c|c|}
\hline \multicolumn{19}{|l|}{ Otros taxones } \\
\hline Rubus sp. & 2 & 1 & 1 & 1 & 1 & + & 1 & 1 & + & 1 & 1 & 1 & 1 & 2 & + & + & 2 & + \\
\hline Brachypodium rupestre & 3 & + & 3 & 1 & 2 & 2 & 3 & 2 & 1 & 2 & 2 & . & 4 & 1 & 1 & 1 & . & 1 \\
\hline Oenanthe crocata & . & + & + & 1 & + & + & 1 & 1 & + & 2 & + & . & + & 1 & 1 & + & 1 & 1 \\
\hline Heracleum sphondylium & 1 & . & 1 & . & 1 & 1 & 1 & 1 & + & 1 & 1 & . & 1 & . & + & + & + & . \\
\hline Dactylis glomerata & 1 & . & . & + & + & 1 & 1 & 1 & + & 1 & + & . & 1 & 1 & $r$ & + & . & . \\
\hline Rumex acetosa & + & . & 1 & . & . & + & + & + & + & + & 1 & . & + & 1 & . & + & + & . \\
\hline Umbilicus rupestris & + & + & + & + & + & + & + & . & + & . & 1 & . & + & . & + & + & . & . \\
\hline Pteridum aquilinum & 2 & 1 & + & . & + & 1 & 1 & 2 & 1 & . & 1 & . & + & . & . & . & . & + \\
\hline Geranium robertianum & + & + & 1 & + & + & . & + & 1 & + & + & 1 & 1 & . & . & . & . & . & . \\
\hline Ranunculus repens & 1 & . & 1 & . & + & . & + & 1 & . & 1 & . & . & 1 & 1 & . & + & . & + \\
\hline Lamium maculatum & 1 & . & + & . & + & . & + & 1 & · & + & . & . & + & 1 & . & . & . & + \\
\hline Pentaglottis sempervirens & 1 & . & . & $\cdot$ & + & . & . & 1 & . & 1 & + & . & 1 & · & . & . & . & · \\
\hline Cardamine hirsuta & . & . & 1 & . & . & . & . & . & + & 1 & 1 & . & + & · & . & . & + & · \\
\hline Ruscus aculeatus & . & . & . & . & + & . & . & · & . & + & 1 & . & . & . & + & + & . & 1 \\
\hline Lapsana communis & + & . & . & $\cdot$ & . & . & . & 1 & . & + & + & . & + & . & + & . & . & . \\
\hline Digitalis purpurea & · & + & + & + & . & . & $r$ & . & + & · & . & . & . & · & · & + & $\cdot$ & . \\
\hline Polygonum persicaria & . & . & . & + & . & . & . & . & + & · & . & + & . & + & . & . & 2 & · \\
\hline Geum urbanum & . & . & 1 & $\cdot$ & . & . & $\cdot$ & · & 1 & 1 & + & . & 1 & · & · & . & $\cdot$ & . \\
\hline Arrhenatherum bulbosum & + & . & 1 & $\cdot$ & . & . & 1 & 1 & · & · & . & $\cdot$ & 1 & · & . & . & $\cdot$ & · \\
\hline Urtica dioica & 1 & . & . & + & . & . & . & . & . & + & . & . & 1 & . & . & . & 1 & . \\
\hline Veronica chamaedrys & + & . & . & . & . & . & . & 1 & 1 & . & + & . & . & + & . & . & . & . \\
\hline Galium aparine & 1 & . & . & . & . & . & . & 1 & . & + & + & . & + & . & . & . & . & . \\
\hline Apium nodiflorum & . & . & . & . & . & . & . & . & + & + & . & . & . & + & . & . & + & 1 \\
\hline Eupatorium cannabinum & 1 & . & . & + & . & . & . & . & . & . & 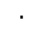 & + & . & . & + & + & . & . \\
\hline Epilobium sp. & . & . & + & . & . & . & . & . & + & . & . & + & + & . & + & . & . & . \\
\hline Narcissus bulbocodium & . & . & . & $\cdot$ & . & + & + & + & . & + & . & . & . & · & . & . & . & $r$ \\
\hline Silene dioica & · & . & . & $\cdot$ & . & . & $\cdot$ & · & . & + & . & + & . & 2 & · & + & $\cdot$ & · \\
\hline Agrostis capillaris & 1 & . & . & $\cdot$ & . & . & . & 1 & 1 & . & . & . & 1 & · & . & . & . & · \\
\hline Angelica sylvestris & . & . & . & $\cdot$ & . & . & $\cdot$ & . & + & . & . & . & + & . & 1 & . & $\cdot$ & 1 \\
\hline Centaurea rivularis & + & . & 1 & $\cdot$ & . & + & . & + & · & . & . & . & . & . & . & . & . & . \\
\hline Prunella vulgaris & + & . & . & $\cdot$ & . & . & $\cdot$ & . & . & + & . & . & + & . & . & $\cdot$ & $\cdot$ & + \\
\hline Senecio aquaticus & . & . & + & $\cdot$ & . & . & + & . & + & . & . & . & . & . & . & . & $\cdot$ & + \\
\hline
\end{tabular}


Taxones presentes en menos de cuatro inventarios:

$\mathrm{E}_{1}+\mathrm{E}_{2}$ : Acacia melanoxylon: + en 6 y 1 en 18; Acer negundo: + en 10; Cytisus scoparius: + en 6; Ficus carica: + en 12 y + en 17; Genista florida: + en 12 y + en 17; Genista florida: + en 6 y + en 16; llex aquifolium: r en 6, + en 15 y + en 16; Juglans regia: $r$ en 1 y r en r en 12; Ligustrum ovalifolium: 1 en 14; Pinus pinaster. 1 en 6; Prunus spinosa: + en 10 y 1 en 18; Quercus pyrenaica: r en 6, + en 11 y + en 13; Robinia pseudoacacia: $r$ en 9 y 2 en 10; Rosa micrantha: 1 en 8 y + en 16; Viburnum opulus: 1 en 9. Taxones característicos de asociación y unidades superiores: Arum italicum: + en 10, + en 13 y + en 15; Circaea lutetiana: + en 9, + en 12 y + en 15; Humulus lupulus: 1 en 13, 1 en 17 y + en 18; Solanum dulcamara: + en 9, + en 11 y 1 en 13; Symphytum tuberosum: + en 10 y + en 11 . Taxones característicos de Quercetea robori-petraeae y Carpino-Fagetea: Dryopteris filix-mas: 1 en 3; Poa nemoralis: + en 6; Potentilla sterilis: 1 en $8,+$ en 9 y + en 11; Tamus communis: + en 9 y + en 18; Otros taxones: Alliaria petiolata: 1 en 10 y + en 11; Anarhinum duriminum: + en 9; Anthoxanthum amarum: + en 3 y + en 9; Anthoxanthum odoratum: + en 6 y + en 15; Asphodelus sp.: + en 13; Bambusa sp.: 2 en 18; Bidens sp.: + en 13 y + en 18; Bidens tripartita: + en 9; Bromus sterilis: + en 1; Caltha palustris: + en 7 y + en 14; Calystegia sepium: 1 en 9, + en 10 y + en 17; Campanula lusitanica: + en 8; Carex binervis: + en 4; Carex lusitanica: + en 13; Carex pilulifera: + en 2; Chelidonium majus: $r$ en 1, 1 en 10 y + en 13; Cirsium filipendulum: + en 6; Cirsium palustre: + en 7 y + en 14; Clematis vitalba: 1 en 10 y 1 en 17; Coincya setigera: + en 5, 1 en 10 y + en 13; Conyza bonariensis: + en 10 y + en 18; Crocus serotinus: + en 8; Cyperus eragrostis: + en 17; Equisetum arvense: 1 en 10 y + en 16; Festuca gr. rubra: + en 6, r en 16 y + en 18; Filipendula ulmaria: + en 9 y + en 18; Fragaria vesca: + en 3; Fumaria sp.: + en 10; Galeopsis tetrahit: + en 13; Galium mollugo: 1 en 1 y + en 7; Galium palustre: + en 3 y 1 en 14; Geranium columbinum: + en 8; Geranium dissectum: + en 8 y + en 11; Geranium lucidum: + en 10, + en 11 y 1 en 13; Geranium purpureum: 1 en 1, 1 en 8 y 1 en 13; Glandora prostrata: + en 6 y r en 16; Glechoma hederácea: + en 10 y + en 11; Holcus lanatus: 1 en 13; Hypericum sp.: + en 3; Hypericum tetrapterum: 1 en 8; Juncus effusus: + en 3 y + en 14; Juncus sp.: r en 8; Lamium purpureum: + en 13; Limniris pseudacorus: 1 en 13 y 1 en 14; Lotus corniculatus: r en 9; Luzula campestris: + en 3; Lycopus europaeus: + en 9 y + en 18; Lysimachia vulgaris: 1 en 8, 1 en 9 y + en 13; Lythrum salicaria: 1 en 9, 1 en 17 y + en 18; Mentha aquatica: + en 18; Mentha suaveolens: 1 en 8 y + en 12; Molinia caerulea: 1 en 8 y + en 12; Molinia caerulea: 1 en 6 y + en 9; Peucedanum gallicum: + en 1; Phalaris arundinacea: 1 en 9 y + en 18; Phytolacca americana: + en 18; Phytolacca americana: + en 18; Picris hieracioides: + en 15 y + en 17; Plantago lanceolata: + en 10; Poa pratensis: + en 10; Polygonum sp.: + en 1, 1 en 13 y + en 18; Polypodium cambricum: 1 en 9; Potentilla erecta: + en 3, + en 6 y + en 8; Prunella grandiflora: 1 en 6; Ranunculus flammula: + en 18; Rumex conglomeratus: + en 13; Rumex obtusifolius: + en 8 , + en 10 y 1 en 13; Samolus valerandi: + en 18; Saponaria officinalis: 1 en 10 y 2 en 13; Scrophularia auriculata: + en 4, + en 10 y + en 17; Scrophularia herminii: + en 1; Silene latifolia: + en 1 y + en 13; Sonchus oleraceus: + en 18; Sparganium neglectum: + en 13; Spergularia media: + en 10; Stellaria media: + en 9; Taraxacum gr. officinale: + en 3, + en 6 y r en 8; Tradescantia fluminensis: 2 en 18; Tritonia x crocosmiflora: 1 en 10, 1 en 15 y + en 16; Ulex minor. + en 6; Valeriana dioica: + en 3 y 2 en 14; Valeriana officinalis: + en 1 y + en 6; Vincetoxicum nigrum: + en 9; Vitis sp.: + en 9 y + en 12.

\section{Procedencia de los inventarios:}

1: Ou: O Carballiño, Arcos, Río Arenteiro (576/4699); 2: Po: Lalín, Cadrón, margen izquierda del Río Arnego (577/4733); 3: Lu: Antas de Ulla, Río Ulla, aguas abajo de Ponte da Mercé (588/4741); 4: C: Santiago de Compostela, Grixoá, Rego de Gato Fero (537/4755); 5: Ou: Avión, entre Avión y Serra, margen izquierda del Río Valdeiras (562/4691); 6: Po: Covelo, Chicoreiros, Río Tea (554/4680); 7: Ou: Piñor, A Canda, entre Porto do Souto y Cotelas, Río Silvaboa (583/4704); 8: Lu: Carballedo, Vilaverde, Río Búbal (599/4706); 9: Po: A Estrada, Vea, Couto do Couso, margen izquierda del Río Ulla (563/4732); 10: Ou: Leiro, Barciamedelle, A Ponte, margen izquierda do Río Avia (572/4689); 11: Ou: Celanova, O Freixo, Río Arnoia, margen izquierda aguas arriba de Ponte Freixo (585/4672); 12: Ou: A Peroxa, Río Búbal, aguas arriba de la aceña de Os Peares (603/4701); 13: Ou: Allariz, Allariz, Río Arnoia (600/4672); 14: C: Santiago de Compostela, A Escarabuña, Rego dos Vilares (537/4752); 15: C: Boiro, Río Beluso, entre Bealo y Boimazán (513/4727); 16: Ou: Ribadavia, Prexigueiro, Río Cerces, aguas debajo de la carretera a Salvaterra (586/4678); 17: Lu: Pantón, Río Miño, margen izquierda entre la presa de Os Peares y Os Peares (604/4701); 18: Po: Tomiño, Goián, Río Miño (520/4643). 
Tabla Ila. Alisedas galaico-portuguesas (Senecioni bayonnensis-Alnetum glutinosae) variante típica, subvariante mesotrofa, facies típica

\begin{tabular}{|c|c|c|c|c|c|c|c|c|c|c|c|c|c|c|c|c|c|c|c|c|c|c|}
\hline $\mathrm{N}^{0}$ de orden & 1 & 2 & 3 & 4 & 5 & 6 & 7 & 8 & 9 & 10 & 11 & 12 & 13 & 14 & 15 & 16 & 17 & 18 & 19 & 20 & 21 & 22 \\
\hline Altitud (m) & 310 & 355 & 345 & 415 & 430 & 380 & 565 & 155 & 295 & 155 & 355 & 115 & 145 & 240 & 265 & 175 & 235 & 160 & 45 & 335 & 335 & 45 \\
\hline Pendiente $\left({ }^{\circ}\right)$ & $<2$ & 4 & 2 & $<2$ & 2 & 2 & 8 & 2 & 6 & 4 & 4 & $<2$ & 4 & 5 & 4 & 4 & 6 & 6 & $<2$ & $<2$ & 6 & 4 \\
\hline Orientación & SW & WSW & SSE & S & $\mathrm{NE}$ & W & SE & S & NW & NNW & NNW & SW & SW & WSW & W & W & NW & NW & $\mathrm{NE}$ & SW & WNW & SW \\
\hline Altura de copas (m) & 14 & $12-20$ & 20 & 14 & 14 & 16 & 22 & 11 & $6-14$ & 16 & 16 & 18 & 16 & 22 & 12 & 12 & 10 & 20 & 14 & 20 & 26 & 24 \\
\hline Cob. $E_{1}(\%)$ & 100 & 90 & 90 & 100 & 90 & 90 & 90 & 100 & 90 & 100 & 95 & 90 & 100 & 100 & 90 & 100 & 90 & 95 & 90 & 100 & 95 & 90 \\
\hline Cob. $E_{2}(\%)$ & 20 & 25 & 20 & 30 & 20 & 40 & 15 & 30 & 50 & 15 & 30 & 10 & 25 & 35 & 20 & 30 & 25 & 25 & 25 & 25 & 30 & 20 \\
\hline Cob. $\mathrm{E}_{3}(\%)$ & 90 & 75 & 95 & 95 & 100 & 95 & 80 & 100 & 90 & 95 & 95 & 95 & 90 & 95 & 95 & 95 & 90 & 85 & 90 & 95 & 85 & 90 \\
\hline Área $\left(\mathrm{m}^{2}\right)$ & 300 & 500 & 300 & 300 & 300 & 300 & 240 & 400 & 600 & 400 & 600 & 300 & 350 & 300 & 300 & 360 & 450 & 300 & 440 & 400 & 400 & 300 \\
\hline $\mathrm{N}^{0}$ de taxones & 53 & 70 & 45 & 37 & 42 & 44 & 39 & 49 & 62 & 59 & 62 & 55 & 48 & 44 & 47 & 44 & 50 & 46 & 46 & 60 & 35 & 41 \\
\hline \multicolumn{23}{|c|}{$E_{1}(>4,0 \mathrm{~m})+E_{2}(>1,5-4,0 \mathrm{~m})$} \\
\hline Alnus glutinosa & 4 & 4 & 4 & 4 & 5 & 4 & 3 & 4 & 3 & 3 & 4 & 4 & 4 & 4 & 4 & 3 & 3 & 3 & 3 & 5 & 4 & 4 \\
\hline Salix atrocinerea & 2 & + & . & 2 & 1 & 1 & 1 & 3 & 2 & 2 & 2 & 2 & 2 & 1 & 2 & 3 & 2 & 2 & 2 & 1 & . & . \\
\hline Quercus robur & 1 & 2 & 1 & + & + & 1 & ${ }^{\circ}$ & 2 & 3 & 2 & 1 & + & 1 & + & . & + & $r$ & . & 1 & 1 & 2 & 1 \\
\hline Sambucus nigra & 2 & 1 & 2 & ${ }^{\circ}$ & . & 1 & . & . & ${ }^{\circ}$ & . & 1 & 1 & + & 1 & . & 1 & 1 & + & 1 & 1 & . & 1 \\
\hline Laurus nobilis & . & . & . & . & . & . & . & 1 & . & 1 & 1 & $r$ & 2 & 2 & 1 & 2 & 1 & + & 1 & 1 & . & 1 \\
\hline Frangula alnus & . & + & 1 & 2 & + & . & . & 1 & + & + & + & . & 1 & + & . & . & . & . & . & . & . & + \\
\hline Corylus avellana & . & 3 & . & . & . & . & + & . & 1 & 3 & 2 & . & . & 2 & 1 & . & 2 & 2 & . & . & 1 & . \\
\hline Crataegus monogyna & . & 1 & . & 1 & . & 2 & . & + & . & 1 & 1 & & 1 & . & . & . & . & . & . & 1 & 1 & + \\
\hline Castanea sativa & . & 1 & . & . & . & . & 1 & . & + & 2 & . & & + & . & . & . & . & . & . & . & 1 & 1 \\
\hline Ilex aquifolium & . & + & . & . & . & + & . & . & + & 1 & . & . & . & 1 & 1 & . & . & . & . & . & . & . \\
\hline Ligustrum ovalifolium & 1 & 1 & . & . & . & + & & . & . & . & + & . & . & . & . & . & . & . & . & . & . & 1 \\
\hline
\end{tabular}

$\mathrm{E}_{3}(<1,5 \mathrm{~m})$ :

Taxones diferenciales frente a Valeriano-Alnetum e Hyperico-Alnetum

Narcissus cyclamineus

Hyacinthoides paivae

\begin{tabular}{|c|c|c|c|c|c|c|c|c|c|c|c|c|c|c|c|c|c|c|c|c|c|c|}
\hline \multicolumn{23}{|c|}{ Taxones diferenciales frente a Hyperico-Alnetum } \\
\hline Hedera hibernica & 1 & 2 & 2 & 1 & 2 & 2 & 2 & 1 & 1 & 2 & 2 & 1 & 3 & 2 & 2 & 2 & 2 & 3 & 3 & 1 & 1 & 2 \\
\hline Viola riviniana & . & 1 & 1 & 1 & 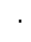 & + & . & + & 1 & 1 & 1 & 1 & 1 & + & + & . & + & 1 & 1 & 1 & 1 & + \\
\hline Osmunda regalis & . & 1 & · & 2 & 3 & 1 & · & 1 & 2 & + & . & + & + & + & 1 & + & 1 & . & . & · & · & 1 \\
\hline Omphalodes nitida & . & 1 & 1 & 1 & + & 1 & · & · & 1 & 1 & + & + & . & + & · & · & . & . & . & 1 & + & · \\
\hline Deschampsia subtriflora & + & + & + & . & 1 & + & 1 & . & + & . & + & + & . & 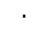 & + & . & . & . & . & + & . & . \\
\hline Betula pubescens & 2 & . & 2 & 2 & 1 & 1 & . & . & . & 1 & . & . & + & 1 & . & . & 1 & . & . & 1 & . & . \\
\hline Carex reuteriana & 1 & 1 & . & 2 & . & . & 1 & + & 1 & . & + & . & . & . & . & . & . & . & . & . & $\mathrm{r}$ & . \\
\hline Caltha palustris & + & . & 1 & . & 1 & . & + & . & . & + & . & . & . & . & + & . & + & . & . & + & . & . \\
\hline Pyrus cordata & . & + & . & + & 1 & . & + & . & 2 & 1 & . & . & . & . & . & . & . & . & . & 2 & . & . \\
\hline Viola palustris & . & 1 & 1 & 1 & + & . & 1 & . & + & . & . & . & . & . & . & . & . & . & . & + & . & . \\
\hline Carex laevigata & . & . & + & . & + & . & + & . & . & + & . & . & . & . & + & . & + & . & . & + & . & . \\
\hline Erica arborea & + & . & . & + & 1 & . & . & + & 1 & . & + & . & . & . & . & . & . & . & . & . & . & . \\
\hline Aquilegia dichroa & . & 1 & . & . & . & . & . & . & + & + & + & . & 1 & . & . & + & . & . & . & . & . & . \\
\hline Allium victorialis & . & 2 & . & . & . & . & 2 & . & . & . & . & . & . & 1 & . & . & . & . & . & . & . & . \\
\hline Saxifraga spathularis & . & . & . & . & 1 & 1 & . & . & . & . & . & . & . & . & . & . & . & . & . & . & + & . \\
\hline Narcissus asturiensis & + & . & . & . & . & + & . & . & . & . & . & . & . & . & . & . & . & . & . & 1 & . & . \\
\hline Narcissus triandrus & . & . & . & . & . & . & + & . & 1 & . & . & . & . & . & . & . & . & . & . & . & . & . \\
\hline Peucedanum lancifolium & . & . & . & . & . & . & . & . & + & . & . & . & . & . & . & . & + & . & . & . & . & . \\
\hline Euphorbia hyberna & . & 1 & . & . & . & . & . & . & . & . & . & . & . & . & . & . & . & . & . & . & . & . \\
\hline Conopodium majus & . & + & . & . & . & . & 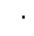 & . & . & . & . & . & . & . & . & . & . & . & . & . & . & . \\
\hline Physospermum cornubiense & . & . & . & . & . & . & . & . & + & . & . & . & . & . & . & . & . & . & . & . & . & . \\
\hline Vaccinium myrtillus & $\cdot$ & $\cdot$ & · & . & · & . & - & · & . & . & . & . & . & + & . & . & . & . & . & . & . & . \\
\hline \multicolumn{23}{|c|}{ Taxones diferenciales de subvariante mesotrofa } \\
\hline Fraxinus excelsior & 1 & 2 & 1 & 2 & 1 & + & 2 & . & . & 1 & 1 & . & + & + & 1 & 2 & 3 & 3 & 3 & 1 & 1 & 2 \\
\hline Veronica montana & . & . & . & . & . & . & . & . & . & + & 1 & 2 & 2 & . & . & + & . & 3 & 1 & + & + & . \\
\hline Angelica major & 2 & . & . & . & + & + & + & . & 1 & . & . & . & . & . & . & 1 & 1 & . & 1 & + & . & . \\
\hline Lysimachia nemorum & . & . & . & - & . & . & 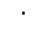 & . & . & + & . & . & . & + & + & . & 1 & 1 & + & . & + & 1 \\
\hline Stachys sylvatica & . & . & . & . & . & . & . & + & . & . & . & 1 & + & . & . & + & . & . & + & . & . & . \\
\hline Mercurialis perennis & . & . & . & - & . & . & . & . & . & + & 2 & 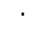 & 1 & 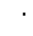 & . & . & . & . & . & . & . & . \\
\hline Pulmonaria longifolia & . & . & . & . & . & . & . & . & 1 & + & . & . & 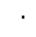 & 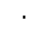 & . & . & . & . & . & . & . & . \\
\hline Potentilla montana & . & . & · & · & . & . & · & · & 1 & . & + & $\cdot$ & . & 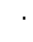 & . & . & . & . & . & · & · & . \\
\hline Ornithogalum pyrenaicum & $\cdot$ & $\cdot$ & · & · & · & · & . & · & . & . & . & $\cdot$ & . & 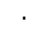 & . & . & . & + & 1 & · & . & 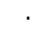 \\
\hline Carex sylvatica & 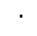 & . & . & . & . & . & . & . & & + & . & 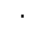 & 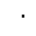 & . & . & . & . & . & . & . & . & + \\
\hline
\end{tabular}




\begin{tabular}{|c|c|c|c|c|c|c|c|c|c|c|c|c|c|c|c|c|c|c|c|c|c|c|}
\hline $\mathrm{N}^{0}$ de orden & 1 & 2 & 3 & 4 & 5 & 6 & 7 & 8 & 9 & 10 & 11 & 12 & 13 & 14 & 15 & 16 & 17 & 18 & 19 & 20 & 21 & 22 \\
\hline \multicolumn{23}{|c|}{ Taxones característicos de asociación y unidades superiores } \\
\hline Athyrium filix-femina & 1 & 2 & 2 & 1 & 1 & 2 & 1 & 2 & + & 3 & 1 & 1 & 1 & 1 & + & 1 & + & 2 & + & 1 & 2 & 1 \\
\hline Lonicera hispanica & 1 & 1 & 2 & 1 & 2 & 1 & 1 & 1 & 1 & 1 & 1 & 1 & 1 & 1 & 1 & 1 & 1 & 1 & 1 & 1 & 1 & 1 \\
\hline Ranunculus ficaria & 2 & 1 & 1 & 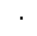 & 2 & 2 & + & 3 & 2 & 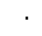 & 2 & 2 & 1 & 1 & 1 & 1 & 3 & 2 & 2 & 2 & + & 1 \\
\hline Euphorbia dulcis & . & 2 & 1 & 1 & 1 & 1 & 1 & + & 2 & 3 & 2 & . & 1 & + & + & + & 1 & 2 & + & 1 & 2 & 1 \\
\hline Dryopteris affinis & 1 & 1 & 1 & 1 & 1 & 1 & 1 & 1 & . & 1 & + & . & 1 & 1 & 1 & 1 & 1 & + & + & 1 & 2 & 2 \\
\hline Primula acaulis & . & 1 & + & + & 2 & 2 & 1 & 2 & 2 & 1 & 1 & . & 1 & + & $\cdot$ & 1 & 1 & 2 & 1 & 2 & + & + \\
\hline Blechnum spicant & + & 1 & 2 & 1 & 2 & 1 & 1 & + & + & 1 & 1 & . & . & 1 & 1 & . & + & + & . & + & 1 & 1 \\
\hline Senecio nemorensis & 2 & + & . & 3 & 1 & 1 & 1 & 2 & + & . & . & + & . & . & . & 1 & . & . & + & 1 & . & . \\
\hline Carex remota & . & 1 & . & . & . & . & . & + & . & + & + & + & + & . & 1 & + & . & . & 1 & + & + & + \\
\hline Hypericum androsaemum & . & + & . & . & . & . & . & + & . & 1 & . & + & 1 & . & + & + & + & + & . & + & + & + \\
\hline Polystichum setiferum & . & 1 & + & . & . & . & 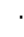 & . & . & . & 1 & . & . & 1 & + & + & . & 3 & 1 & . & + & 1 \\
\hline Myosotis martini & + & + & + & . & . & . & . & . & 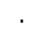 & 1 & 1 & $\cdot$ & . & . & + & . & . & + & . & 1 & . & . \\
\hline Allium scorzonerifolium & . & + & . & . & + & . & . & + & + & . & 4 & . & . & . & . & . & . & + & . & . & . & . \\
\hline Carex pendula & . & . & . & . & . & . & . & . & . & . & . & 1 & 1 & + & + & + & . & . & 2 & . & . & . \\
\hline Arum italicum & . & . & + & . & . & . & . & . & . & . & . & . & . & + & . & + & . & 1 & 2 & . & . & . \\
\hline Tamus communis & . & 1 & + & . & . & . & . & . & . & 1 & + & . & . & . & . & 1 & . & . & . & . & . & . \\
\hline Brachypodium sylvaticum & . & + & · & . & . & . & 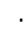 & . & . & 1 & $\cdot$ & $\cdot$ & $\cdot$ & $\cdot$ & $\cdot$ & $\cdot$ & . & + & 1 & . & + & . \\
\hline
\end{tabular}

\begin{tabular}{|c|c|c|c|c|c|c|c|c|c|c|c|c|c|c|c|c|c|c|c|c|c|c|}
\hline \multicolumn{23}{|c|}{ Taxones característicos de Quercetea robori-petraeae y Carpino-Fagetea } \\
\hline Holcus mollis & 1 & 1 & 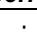 & 3 & 1 & + & + & 1 & 1 & + & + & + & 1 & 1 & & + & 1 & + & & 2 & + & 1 \\
\hline Ajuga reptans & . & 1 & + & 1 & . & 1 & + & . & 1 & 1 & + & + & 1 & + & + & + & + & + & & + & + & 1 \\
\hline Dryopteris dilatata & 1 & + & 1 & . & . & + & . & . & + & . & + & + & 1 & 2 & 1 & + & 1 & + & & - & 1 & 2 \\
\hline Polypodium vulgare & . & + & . & . & . & 1 & + & + & + & + & 1 & . & 1 & . & 1 & + & 1 & 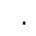 & & 1 & + & + \\
\hline Teucrium scorodonia & + & + & + & 1 & . & + & + & + & + & + & + & + & . & + & . & . & + & . & & 1 & . & . \\
\hline Oxalis acetosella & . & + & . & . & . & + & 1 & . & . & 1 & 1 & . & . & + & + & 1 & + & 1 & . & . & 1 & 2 \\
\hline Hyacinthoides non-scripta & . & + & . & . & 1 & 1 & . & . & . & . & 1 & . & + & + & & 1 & 1 & 1 & + & . & . & + \\
\hline Potentilla sterilis & . & . & . & . & . & . & . & 1 & . & 1 & . & + & + & . & . & . & . & + & 1 & . & + & + \\
\hline Euphorbia amygdaloides & + & + & . & . & + & 1 & . & + & + & 1 & + & . & . & . & . & . & . & . & . & . & . & . \\
\hline Stellaria holostea & + & . & . & . & . & . & . & . & . & + & . & + & . & . & 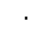 & . & . & . & + & + & 1 & + \\
\hline Anemone nemorosa & . & 2 & . & . & . & . & . & . & 2 & 2 & 2 & . & . & . & . & . & . & . & . & . & 1 & . \\
\hline Crepis lampsanoides & . & + & . & 1 & . & . & . & . & . & . & + & . & . & + & + & . & . & . & . & . & . & . \\
\hline Stachys officinalis & $\cdot$ & + & . & . & 1 & . & . & . & 1 & . & · & · & . & · & . & · & $\cdot$ & $\cdot$ & . & 1 & · & . \\
\hline \multicolumn{23}{|l|}{ Otros taxones } \\
\hline Rubus sp. & 1 & + & 2 & 1 & 3 & 1 & 1 & 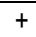 & 1 & 1 & 1 & 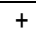 & 1 & 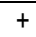 & 2 & 1 & 1 & 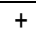 & 1 & 1 & . & 1 \\
\hline Oenanthe crocata & 1 & + & 1 & 1 & 1 & 1 & + & + & 1 & 1 & 2 & + & 1 & + & + & 1 & 1 & + & 2 & + & . & . \\
\hline Brachypodium rupestre & 1 & + & 1 & . & 1 & 1 & 1 & 3 & 3 & 2 & 2 & 2 & 2 & 1 & 1 & . & 2 & . & 2 & 1 & . & 1 \\
\hline Heracleum sphondylium & 1 & + & . & + & . & . & 1 & 1 & + & 1 & 1 & 2 & 1 & + & + & 1 & 1 & . & 2 & 1 & . & . \\
\hline Geranium robertianum & 1 & + & + & . & . & . & + & . & + & + & + & . & + & + & + & + & + & 1 & . & + & . & . \\
\hline Ranunculus repens & 1 & + & 1 & + & . & + & . & . & . & . & + & + & + & . & + & . & + & + & + & + & . & . \\
\hline Cardamine pratensis & 1 & + & 1 & . & + & . & . & 1 & + & . & + & + & + & . & . & . & 1 & + & . & + & . & . \\
\hline Dactylis glomerata & . & . & + & + & $\mathrm{r}$ & + & + & + & 1 & . & + & . & . & . & . & . & 1 & . & + & + & . & + \\
\hline Lamium maculatum & 1 & . & 2 & + & . & . & . & . & . & . & + & . & + & . & 1 & 1 & + & . & 1 & + & . & + \\
\hline Scrophularia auriculata & + & . & + & . & . & . & . & 1 & . & + & . & + & + & . & + & . & . & + & + & + & . & . \\
\hline Angelica sylvestris & . & . & . & . & . & . & . & 1 & 1 & 1 & 2 & 1 & + & + & + & . & . & + & . & . & . & . \\
\hline Pteridum aquilinum & + & . & . & 1 & . & . & + & . & 1 & + & . & + & + & . & . & . & . & . & 1 & + & . & . \\
\hline Umbilicus rupestris & + & + & . & . & . & + & . & + & . & . & + & . & . & . & + & + & 1 & . & . & + & . & . \\
\hline Limniris pseudacorus & + & . & + & . & . & . & . & + & . & . & . & + & + & + & + & + & + & . & . & . & . & . \\
\hline Prunella vulgaris & + & . & . & . & + & . & . & + & . & + & + & + & . & . & + & . & . & . & . & + & . & . \\
\hline Filipendula ulmaria & . & 1 & . & . & 1 & 1 & . & . & 1 & 1 & 2 & 1 & 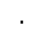 & . & . & . & . & . & . & . & . & . \\
\hline Geum urbanum & . & . & . & . & . & . & . & . & . & + & + & 1 & + & . & . & + & . & 1 & 1 & . & . & . \\
\hline Digitalis purpurea & + & . & 1 & . & . & . & . & + & + & . & . & . & + & . & + & + & . & . & . & . & . & . \\
\hline Urtica dioica & . & . & 1 & . & . & . & . & + & . & . & . & + & . & . & . & . & + & + & + & + & . & . \\
\hline Galium aparine & + & . & 1 & . & . & . & . & . & . & . & 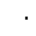 & 1 & . & . & 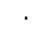 & + & . & . & + & + & . & . \\
\hline Cardamine hirsuta & . & . & 1 & . & . & . & . & + & . & . & . & + & . & . & + & . & . & . & + & + & . & . \\
\hline Calystegia sepium & . & . & . & + & . & . & . & + & . & . & 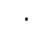 & + & . & . & . & . & 1 & . & 1 & . & . & . \\
\hline Senecio aquaticus & + & + & + & . & . & . & . & . & . & 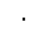 & . & . & 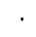 & . & & & + & & & + & . & \\
\hline Rumex acetosa & + & . & . & . & . & . & . & + & . & . & + & + & . & . & . & . & + & . & . & . & . & . \\
\hline Ruscus aculeatus & $\cdot$ & + & . & . & . & . & . & . & . & 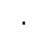 & . & . & 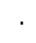 & + & & - & . & + & . & . & + & + \\
\hline Valeriana dioica & . & . & . & . & . & . & 1 & . & . & 1 & 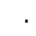 & 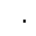 & 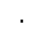 & 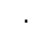 & & 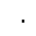 & 1 & 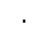 & & + & . & . \\
\hline Rumex obtusifolius & . & . & . & . & . & . & . & + & . & . & + & 1 & . & . & . & . & - & . & . & + & . & . \\
\hline Silene dioica & . & . & . & . & . & . & . & . & . & . & + & . & . & . & + & . & 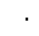 & . & + & 1 & . & . \\
\hline Eupatorium cannabinum & & + & . & . & . & . & . & . & + & 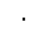 & . & + & . & . & & . & & + & & & . & \\
\hline Mentha aquatica & + & . & . & . & . & . & . & . & . & . & . & + & + & . & & $r$ & ${ }^{\circ}$ & . & . & . & . & \\
\hline
\end{tabular}


Taxones presentes en menos de cuatro inventarios:

$\mathrm{E}_{1}+\mathrm{E}_{2}$ : Acer pseudoplatanus: 1 en 11; Cytisus scoparius: 1 en 9; Rosa gr. canina: + en 8, + en 10 y + en 12; Ulmus glabra: 2 en 18 y 1 en 21; Viburnum opulus: 2 en 2. Taxones característicos de asociación y unidades superiores: Circaea lutetiana: 2 en 12; Solanum dulcamara: 1 en 1 y + en 12. Taxones característicos de Quercetea robori-petraeae y Carpino-Fagetea: Aquilegia vulgaris: + en 6 y + en 7; Dryopteris filix-mas: + en 2; Lathyrus linifolius: + en 9 y + en 10; Luzula sylvatica: 2 en 2 y 1 en 21; Melampyrum pratense: + en 7; Melittis melissophyllum: + en 2; Phyllitis scolopendrium: + en 16 y + en 22; Polygonatum verticillatum: 1 en 2. Otros taxones: Anthoxanthum amarum: + en 5 y + en 9; Apium nodiflorum: + en 1; Asphodelus lusitanicus: + en 5 y 1 en 20; Carex binervis: + en 5; Carex lusitanica: + en 22; Carex pilulifera: + en 6; Centaurea rivularis: 1 en 9 y + en 17; Chrysosplenium oppositifolium: 1 en 3,1 en 18 y 1 en 22; Cirsium palustre: + en 9 y + en 17; Crocus serotinus: + en 20; Cystopteris sp.: +en 18; Daboecia cantabrica: + en 1; Epilobium sp.: + en 1; Festuca gr. rubra: + en 9; Galium palustre: + en 1 y + en 8; Glechoma hederacea: + en 19; Glyceria fluitans: + en 12; Hypericum tetrapterum: + en 4; Juncus effusus: + en 1 y + en 8; Luzula campestris: 1 en 6; Lycopus europaeus: + en 2 y + en 12; Lythrum salicaria: 1 en 9 y + en 12; Narcissus bulbocodium: + en 1, + en 5 y + en 20; Pentaglottis sempervirens: 1 en 3, 1 en 18 y + en 20; Polygonatum odoratum: + en 2 y 1 en 10; Polygonum persicaria: $r$ en 4; Polypodium interjectum: + en 14; Potentilla erecta: + en 9 y + en 11; Rumex sp.: r en 19; Scirpus setaceus: + en 15; Sibthorpia europea: + en 15; Sonchus oleraceus: + en 12; Sparganium neglectum: + en 1 y + en 2; Taraxacum gr. officinale: + en 11 y + en 12; Tritonia x crocosmiflora: 1 en 18; Ulex minor. + en 1 y + en 9; Valeriana montana: 1 en 22; Veronica chamaedrys: + en 12.

Procedencia de los inventarios:

1: C: Frades, Abellá, Río Samo, entre O Muiño Grande dos Baltares y Ponte Carballa (557/4771): 2: C: Vilasantar/Boimorto, Río Tambre, augas arriba de la Central de S. Pedro (570/4797); 3: C: Arzúa, Burres, entre A Igrexa y Salmonte, Rego Lengüello (563/4751); 4: Lu: Guntín, entre Castelo do Bispo y Guntín, Río Ferreira (604/4749); 5: C: Curtis, Río Deo, augas arriba de su confluencia con el Río Mandeo (579/4782); 6: C: Aranga, entre A Castellana y Os Ferreiros, Rego da Ribeira (580/4782); 7: C: Melide, A Capela, Rego Pequeño (583/4758); 8: C: Baio, Sta. Irene, Río Grande, marxe dereita (500/4777); 9: C: Santa Catalina de Armada, Grixoá, entre Armán y Brandomil, Río Xallas (507/4762); 10: C: Coristanco, O Couso, Curras de Abaixo, Rego da Rebuxenta (518/4777); 11: C: Santa Comba, Bazar, Boimente, Río Xallas (521/4768); 12: C: Carballo, Bértoa, entre Queo y O Añón de Berdillo, Río Anllóns (528/4786); 13: C: A Laracha, Vilaño, Amboade, Río do Acheiro (531/4785); 14: C: A Laracha, Golmar, San Pantaión, Río do Acheiro (535/4785); 15: C: A Laracha, entre O Seixoso y Gosende, Río do Acheiro (536/4785); 16: C: A Laracha, A Torre, Río Grande (539/4787); 17: C. Meirama, Os Vilares, Rego do Porto dos Carros (544/4785); 18: C: Touro, Prevediños, O Castro, Rego do Noallo (551/4744); 19: C: Abegondo, As Tabóas, margen derecha del Río Barcés (557/4788); 20: C: Mesía, Xanceda, As Corredoiras, Rego do Molar (561/4768); 21: C: OzaCesuras, Filgueira de Barranca, As Nabeiras, margen derecha del Río Mendo (567/4777); 22: C: Miño/Vilarmaior, Rego do Porto das Lapelas, aguas arriba de Ponte da Brea (569/4796). 
Tabla Ilb. Alisedas galaico-portuguesas (Senecioni bayonnensis-Alnetum glutinosae) variante típica, subvariante mesotrofa, facies típica (cont.)

\begin{tabular}{|c|c|c|c|c|c|c|c|c|c|c|c|c|c|c|c|c|c|c|c|c|c|c|}
\hline $\mathrm{N}^{0}$ de orden & 1 & 2 & 3 & 4 & 5 & 6 & 7 & 8 & 9 & 10 & 11 & 12 & 13 & 14 & 15 & 16 & 17 & 18 & 19 & 20 & 21 & 22 \\
\hline Altitud (m) & 275 & 302 & 340 & 505 & 460 & 475 & 275 & 405 & 405 & 470 & 440 & 495 & 175 & 95 & 35 & 95 & 105 & 205 & 165 & 205 & 215 & 115 \\
\hline Pendiente $\left({ }^{\circ}\right)$ & 2 & 6 & 2 & $<2$ & 4 & $<2$ & 2 & $<2$ & $<2$ & 4 & $<2$ & $<2$ & 8 & 4 & 2 & 2 & $<2$ & $<2$ & 4 & 4 & $<2$ & 6 \\
\hline Orientación & $S$ & $\mathrm{~N}$ & WSW & NNW & $\mathrm{N}$ & NW & $\mathrm{NE}$ & SSE & SE & WSW & ENE & SW & $S$ & $S$ & WSW & W & $\mathrm{N}$ & $\mathrm{N}$ & SW & WSW & SW & ENE \\
\hline Altura de copas (m) & 18 & 16 & 24 & 20 & 14 & 18 & 22 & 18 & 12 & 20 & 12 & 14 & 12 & 16 & 18 & 16 & 18 & 16 & 12 & 18 & 12 & 12 \\
\hline Cob. $E_{1}(\%)$ & 90 & 90 & 100 & 95 & 100 & 100 & 95 & 95 & 90 & 100 & 100 & 100 & 100 & 90 & 95 & 90 & 100 & 90 & 100 & 100 & 90 & 100 \\
\hline Cob. $E_{2}(\%)$ & 35 & 25 & 40 & 15 & 20 & 20 & 20 & 30 & 25 & 20 & 20 & 20 & 20 & 20 & 25 & 20 & 25 & 30 & 15 & 15 & 20 & 25 \\
\hline Cob. $E_{3}(\%)$ & 90 & 90 & 95 & 95 & 95 & 95 & 95 & 100 & 95 & 95 & 95 & 95 & 95 & 95 & 95 & 95 & 95 & 95 & 95 & 85 & 100 & 85 \\
\hline Área $\left(\mathrm{m}^{2}\right)$ & 400 & 300 & 300 & 300 & 500 & 500 & 300 & 400 & 200 & 300 & 300 & 300 & 400 & 400 & 500 & 300 & 500 & 400 & 500 & 300 & 300 & 300 \\
\hline $\mathrm{N}^{0}$ de taxones & 57 & 41 & 44 & 55 & 66 & 58 & 63 & 54 & 42 & 53 & 49 & 40 & 44 & 56 & 59 & 57 & 63 & 76 & 64 & 47 & 57 & 49 \\
\hline \multicolumn{23}{|l|}{$E_{1}(>4,0 m)+E_{2}(>1,5-4,0 n$} \\
\hline Alnus glutinosa & 4 & 5 & 4 & 5 & 3 & 5 & 5 & 5 & 5 & 4 & 5 & 4 & 4 & 5 & 4 & 4 & 4 & 3 & 3 & 5 & 3 & 4 \\
\hline Salix atrocinerea & 1 & 1 & 2 & 1 & + & 1 & 1 & . & 1 & 1 & 1 & + & 2 & 1 & 1 & 3 & 2 & 2 & 2 & 1 & 2 & 1 \\
\hline Quercus robur & 1 & 1 & 1 & + & 3 & 1 & + & + & . & 1 & 1 & 1 & 1 & 1 & . & 1 & 1 & 2 & 1 & + & 2 & . \\
\hline Frangula alnus & 1 & + & ${ }^{\circ}$ & + & + & 1 & + & + & 2 & 1 & 1 & 1 & 1 & . & + & 1 & + & + & . & . & . & . \\
\hline Sambucus nigra & . & ${ }^{\circ}$ & . & 1 & 1 & 1 & . & 1 & . & 1 & 1 & 1 & . & . & 1 & 1 & 1 & . & 1 & 1 & + & 1 \\
\hline Corylus avellana & 1 & . & 2 & . & 3 & . & + & . & . & 1 & . & 1 & . & . & 1 & + & 1 & + & 1 & 2 & . & 2 \\
\hline Laurus nobilis & . & 1 & & . & . & . & . & . & . & & . & . & 1 & . & 1 & + & 1 & + & 1 & 1 & & 1 \\
\hline Castanea sativa & . & . & & . & + & + & + & 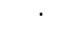 & . & 1 & . & 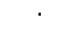 & . & 1 & + & . & . & 1 & 1 & . & & . \\
\hline Crataegus monogyna & 1 & . & & 1 & 1 & . & 1 & 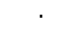 & . & 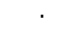 & 1 & 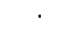 & 1 & . & 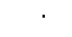 & & 2 & 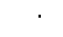 & . & . & 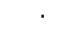 & 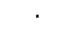 \\
\hline Rosa gr. canina & . & 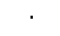 & & + & . & . & . & . & + & & . & 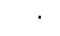 & . & . & + & & & + & . & . & & $\cdot$ \\
\hline
\end{tabular}

\section{$\mathrm{E}_{3}(<1,5 \mathrm{~m}):$}

Taxones diferenciales frente a Valeriano-Alnetum e Hyperico-Alnetum

Narcissus cyclamineus

Fraxinus angustifolia

Faxinus excelsior $x$ F. angustifolia

Hyacinthoides paivae

\section{$\mathrm{E}_{3}(<1,5 \mathrm{~m}):$ \\ Taxones diferenciales frente a Hyperico-Alnetum}

\section{Hedera hibernica}

Omphalodes nitida

Osmunda regalis

Carex reuteriana

Viola palustris

Deschampsia subtriflora

Betula pubescens

Angelica major

Pyrus cordata

Caltha palustris

Erica arborea

Peucedanum lancifolium

Aquilegia dichroa

Saxifraga spathularis

Narcissus triandrus

Euphorbia hyberna

Physospermum cornubiense

\begin{tabular}{|c|c|c|c|c|c|c|c|c|c|c|c|c|c|c|c|c|c|c|c|c|c|}
\hline & & & & & & & & & & & & & & & & & & & & & \\
\hline 2 & 2 & + & 2 & 2 & 2 & 2 & 3 & + & 2 & 2 & 2 & 2 & 1 & 3 & 1 & 2 & 2 & 1 & 2 & 3 & 3 \\
\hline 1 & 1 & + & + & . & 1 & + & 1 & 2 & + & + & & 1 & 1 & 1 & . & . & & + & & + & \\
\hline . & 2 & . & . & . & . & 2 & ${ }^{\prime}$ & 1 & . & ${ }^{\prime}$ & & 1 & 1 & 4 & + & 1 & 1 & + & 2 & + & . \\
\hline 1 & 1 & . & + & 1 & + & 1 & . & 2 & 1 & . & . & 1 & . & 2 & . & . & + & . & . & 1 & . \\
\hline 2 & + & . & . & 1 & + & 1 & . & + & 1 & + & 1 & + & . & 2 & . & . & 1 & . & . & . & . \\
\hline . & . & . & + & + & 1 & + & . & 1 & + & + & + & 1 & . & + & . & . & + & . & . & . & . \\
\hline 1 & . & $\cdot$ & 1 & . & 1 & . & . & 1 & 1 & 1 & 1 & . & . & . & 1 & . & . & . & 1 & · & . \\
\hline . & . & + & . & + & + & . & . & $\cdot$ & + & + & . & $\cdot$ & + & + & . & . & . & + & + & . & 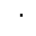 \\
\hline 1 & . & $\cdot$ & + & 1 & + & 1 & . & . & . & . & + & . & ${ }^{\circ}$ & + & . & . & 1 & . & . & . & . \\
\hline 1 & 1 & + & . & 1 & + & + & + & . & . & . & . & . & . & . & . & . & . & . & . & . & . \\
\hline . & + & . & . & . & + & + & . & + & + & . & 1 & . & . & + & . & . & . & . & . & . & . \\
\hline . & . & + & . & . & $r$ & . & . & . & . & . & & + & . & $r$ & . & . & 1 & . & . & . & . \\
\hline . & . & . & . & . & . & . & . & . & 1 & . & & . & + & 1 & . & . & . & + & . & . & . \\
\hline . & + & . & + & . & . & 1 & . & . & . & . & & . & . & . & . & . & . & . & . & . & + \\
\hline . & . & . & . & . & . & . & + & . & . & . & & . & . & + & . & . & . & . & . & . & \\
\hline . & . & . & . & 1 & . & . & . & . & . & . & & . & . & . & . & . & . & . & . & . & \\
\hline . & . & . & . & . & . & + & . & . & . & & & & . & . & . & . & . & . & . & . & \\
\hline
\end{tabular}

Taxones diferenciales de subvariante mesotrofa

\begin{tabular}{|c|c|c|c|c|c|c|c|c|c|c|c|c|c|c|c|c|c|c|c|c|c|c|}
\hline Fraxinus excelsior & 1 & 1 & 2 & . & . & . & $\begin{array}{ccc}+ \\
+\end{array}$ & 1 & 2 & 1 & 2 & 2 & 1 & 1 & . & + & 2 & . & 3 & 2 & . & 2 \\
\hline Stachys sylvatica & 1 & . & . & 1 & + & + & . & + & 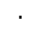 & 1 & . & - & . & . & + & 1 & + & + & + & . & 2 & . \\
\hline Veronica montana & . & . & 1 & . & + & + & . & 1 & . & . & . & . & . & 1 & . & 2 & 1 & . & 2 & . & & 1 \\
\hline Lysimachia nemorum & . & 1 & 1 & . & + & . & + & . & . & . & . & . & . & 1 & . & . & . & . & . & 1 & . & 1 \\
\hline Ornithogalum pyrenaicum & . & . & 1 & + & 1 & . & . & + & + & . & . & . & . & . & . & + & + & . & . & . & . & . \\
\hline Mercurialis perennis & . & . & 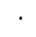 & . & . & . & . & + & ${ }^{\prime}$ & . & . & . & . & 1 & . & . & + & . & 1 & . & . & . \\
\hline Carex sylvatica & + & . & . & . & . & . & . & r. & . & . & . & . & . & . & . & + & & . & . & . & . & . \\
\hline Ranunculus tuberosus & . & . & . & . & . & . & + & . & . & . & . & . & . & + & . & . & . & . & . & . & . & . \\
\hline Pulmonaria longifolia & . & . & . & . & . & . & . & . & . & . & . & . & . & 1 & . & . & ${ }^{\circ}$ & . & . & . & . & . \\
\hline Potentilla montana & . & . & . & . & . & . & . & . & . & . & . & . & . & . & . & . & . & + & . & . & . & . \\
\hline Sanicula europaea & . & . & . & . & . & . & . & . & . & . & . & . & . & . & . & . & & . & . & . & . & 1 \\
\hline
\end{tabular}




\begin{tabular}{|c|c|c|c|c|c|c|c|c|c|c|c|c|c|c|c|c|c|c|c|c|c|c|}
\hline $\mathrm{N}^{0}$ de orden & 1 & 2 & 3 & 4 & 5 & 6 & 7 & 8 & 9 & 10 & 11 & 12 & 13 & 14 & 15 & 16 & 17 & 18 & 19 & 20 & 21 & 22 \\
\hline \multicolumn{23}{|c|}{ Taxones característicos de asociación y unidades superiores } \\
\hline Dryopteris affinis & 1 & 1 & 1 & 1 & 1 & 1 & + & 1 & 1 & + & 1 & 2 & 1 & 1 & + & 1 & + & 1 & 1 & 1 & 1 & 1 \\
\hline Athyrium filix-femina & 2 & 2 & 2 & 2 & 1 & 1 & 1 & 2 & 3 & 2 & 2 & 3 & 2 & 2 & 2 & 2 & + & . & 1 & 1 & 2 & 1 \\
\hline Viola riviniana & 1 & + & + & + & . & + & 1 & 1 & 1 & 1 & 1 & + & 1 & 1 & 2 & + & + & 2 & 1 & + & 1 & + \\
\hline Lonicera hispanica & + & 2 & 1 & . & 1 & 2 & + & $\cdot$ & 1 & 1 & 1 & 2 & 2 & 1 & 1 & 1 & + & 1 & 1 & 1 & 2 & 1 \\
\hline Euphorbia dulcis & 2 & 1 & 1 & 1 & 2 & + & 1 & 2 & . & 1 & 1 & 1 & 2 & 1 & 1 & 1 & 1 & 1 & 1 & . & 1 & 1 \\
\hline Primula acaulis & 2 & + & . & 1 & 1 & + & 1 & 2 & + & 1 & + & 1 & + & 2 & 1 & 1 & 1 & 1 & 1 & + & 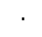 & 1 \\
\hline Ranunculus ficaria & 1 & + & 1 & 2 & 1 & 2 & 1 & 1 & . & 1 & 1 & 1 & 1 & 1 & . & 1 & 1 & . & 2 & 2 & . & 1 \\
\hline Blechnum spicant & + & 2 & $r$ & + & 1 & 1 & 1 & & 1 & 1 & 1 & 3 & 2 & . & + & . & + & + & + & 1 & . & 1 \\
\hline Senecio nemorensis & 2 & 1 & 1 & . & 1 & 2 & 1 & 3 & 2 & 1 & 2 & & . & 1 & 1 & . & 1 & 1 & 1 & . & 1 & . \\
\hline Arum italicum & . & . & + & + & . & . & . & 1 & $\cdot$ & $\cdot$ & + & & + & . & . & 1 & + & . & 1 & + & . & + \\
\hline Myosotis martini & 1 & . & 1 & . & 1 & + & . & 1 & . & 1 & $\cdot$ & & . & . & . & . & . & . & + & . & . & . \\
\hline Carex pendula & . & + & + & . & . & . & . & + & . & . & . & & . & . & . & + & 1 & . & + & . & . & + \\
\hline Hypericum androsaemum & . & + & . & . & . & . & . & $\cdot$ & $\cdot$ & $\cdot$ & $\cdot$ & 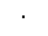 & . & + & . & + & + & . & + & 1 & . & + \\
\hline Circaea lutetiana & 1 & . & + & . & 1 & . & . & $\cdot$ & . & . & $\cdot$ & & . & 2 & . & 1 & . & + & . & . & . & $\cdot$ \\
\hline Polystichum setiferum & . & . & . & . & . & . & + & 1 & $\cdot$ & $\cdot$ & $\cdot$ & . & . & . & . & + & 1 & . & . & 1 & . & 2 \\
\hline Brachypodium sylvaticum & . & . & 1 & . & . & . & 1 & 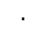 & . & $\cdot$ & $\cdot$ & & . & . & . & . & + & . & . & . & . & + \\
\hline Solanum dulcamara & $\mathrm{r}$ & . & . & . & . & . & . & 1 & . & . & $\cdot$ & . & . & + & . & . & . & . & . & . & 1 & . \\
\hline Carex remota & . & . & 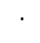 & . & $\cdot$ & 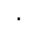 & · & & $\cdot$ & · & $\cdot$ & & $\cdot$ & · & . & + & + & . & + & $\cdot$ & . & + \\
\hline Taxones característicos $\mathrm{d}$ & etea & robo & ipe & eae & Car & ino & Fag & & & & & & & & & & & & & & & \\
\hline Holcus mollis & 2 & 1 & 1 & 1 & 1 & - & + & & 1 & 1 & 1 & 2 & $\cdot$ & + & 1 & . & . & 2 & 1 & 1 & 2 & + \\
\hline Dryopteris dilatata & . & 1 & 1 & + & + & 1 & 1 & 1 & . & + & . & + & 1 & . & + & . & + & + & 1 & 1 & & 2 \\
\hline Teucrium scorodonia & 1 & 1 & . & + & + & 1 & 1 & 1 & 1 & . & 1 & 1 & 1 & + & 1 & . & . & 1 & . & . & 1 & . \\
\hline Polypodium vulgare & + & 1 & + & . & . & + & 1 & 1 & . & + & + & + & . & + & . & . & + & + & + & . & + & 1 \\
\hline Stellaria holostea & . & + & + & 1 & 1 & + & . & 2 & . & . & 1 & 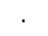 & . & + & + & . & + & + & 1 & + & . & + \\
\hline Euphorbia amygdaloides & + & & . & + & . & + & 1 & 1 & $\cdot$ & + & + & & + & 1 & . & + & + & . & + & . & + & . \\
\hline Ajuga reptans & + & . & . & . & 1 & 1 & 1 & + & 1 & + & . & . & 1 & 1 & 1 & . & . & . & . & . & . & 1 \\
\hline Hyacinthoides non-scripta & . & . & . & 1 & 1 & . & 1 & + & $\cdot$ & . & . & + & . & . & . & . & . & + & 1 & + & . & + \\
\hline Potentilla sterilis & 1 & . & . & + & + & . & . & 1 & . & + & $\cdot$ & 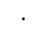 & . & + & . & . & . & + & + & . & . & + \\
\hline Oxalis acetosella & . & . & . & 1 & 1 & . & + & . & $\cdot$ & . & $\cdot$ & 1 & + & . & . & . & $\cdot$ & . & . & + & . & 1 \\
\hline Anemone nemorosa & . & . & 1 & . & 2 & . & 1 & $\cdot$ & . & . & $\cdot$ & . & . & . & . & + & . & + & . & + & . & . \\
\hline Aquilegia vulgaris & 1 & . & . & + & + & . & 1 & + & . & . & . & & . & . & . & + & . & . & . & . & . & . \\
\hline Crepis lampsanoides & . & . & . & . & . & . & 1 & . & + & . & + & & . & . & + & . & . & + & + & . & . & . \\
\hline Stachys officinalis & 1 & . & . & . & . & + & 1 & . & . & . & . & . & . & . & . & . & . & 1 & . & . & 1 & . \\
\hline Tamus communis & + & . & . & . & . & . & + & . & . & . & . & & . & 1 & + & . & . & . & . & + & . & . \\
\hline Luzula sylvatica & $\cdot$ & 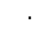 & · & 1 & . & . & 2 & . & $\cdot$ & $\cdot$ & . & 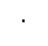 & $\cdot$ & . & 1 & . & . & . & . & $\cdot$ & $\cdot$ & + \\
\hline Otros taxones & & & & & & & & & & & & & & & & & & & & & & \\
\hline Rubus & 1 & 1 & 1 & + & 1 & 1 & + & 1 & 1 & 1 & + & 1 & 2 & + & 1 & 2 & 1 & + & 1 & 2 & + & 1 \\
\hline Oenanthe crocata & 2 & . & + & + & 1 & 1 & 1 & 1 & 1 & 1 & + & 1 & . & 1 & + & 1 & 1 & + & 1 & 1 & 1 & 1 \\
\hline Brachypodium rupestre & 2 & 1 & 1 & 1 & . & 1 & 1 & 4 & 4 & 1 & 1 & & . & 2 & 3 & 3 & 3 & 3 & 2 & 1 & 2 & . \\
\hline Heracleum sphondylium & + & 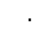 & 1 & 2 & + & 1 & . & + & + & . & + & + & . & + & 1 & 1 & 1 & . & 1 & + & 1 & 2 \\
\hline Geranium robertianum & + & . & + & + & + & + & + & 1 & $\cdot$ & 1 & + & $\cdot$ & . & + & . & 1 & + & + & 1 & + & 1 & + \\
\hline Ranunculus repens & . & . & . & 1 & + & + & . & + & $r$ & + & 1 & . & + & . & + & + & + & + & + & 1 & + & . \\
\hline Dactylis glomerata & . & 1 & . & . & + & 1 & + & . & 1 & 1 & + & + & . & + & . & . & . & 1 & 1 & + & 1 & . \\
\hline Lamium maculatum & + & . & + & 1 & . & 1 & . & + & . & + & + & 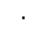 & . & + & . & + & + & $\cdot$ & 1 & . & 1 & . \\
\hline Cardamine pratensis & + & . & 1 & 1 & + & 1 & . & . & . & + & . & . & . & + & . & . & + & . & 1 & 1 & 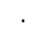 & + \\
\hline Pteridum aquilinum & $r$ & . & . & . & . & 1 & . & + & 1 & + & . & + & + & 1 & + & . & . & + & . & . & 1 & . \\
\hline Cardamine hirsuta & + & . & . & + & . & + & $r$ & . & . & . & + & & . & . & . & + & + & + & 1 & . & 1 & . \\
\hline Umbilicus rupestris & . & + & + & + & . & . & + & + & $\cdot$ & + & . & . & . & . & . & . & + & $\cdot$ & + & 1 & & + \\
\hline Angelica sylvestris & . & . & . & . & . & . & . & + & . & . & . & . & . & 1 & + & 1 & 1 & + & 1 & . & + & + \\
\hline Scrophularia auriculata & . & . & + & . & . & . & + & + & + & . & . & + & . & + & . & + & + & $\cdot$ & . & + & . & . \\
\hline Geum urbanum & . & . & . & + & . & + & . & . & . & . & . & . & . & 1 & . & 1 & 1 & + & . & . & 1 & + \\
\hline Digitalis purpurea & . & . & . & + & . & + & . & . & . & . & + & & + & . & + & . & . & $r$ & . & $\mathrm{r}$ & + & . \\
\hline Filipendula ulmaria & . & . & . & . & 1 & + & 1 & . & . & . & . & . & . & . & . & 1 & 3 & 1 & . & . & 3 & . \\
\hline Silene dioica & . & . & . & . & . & . & . & + & . & . & 2 & $\cdot$ & . & . & + & . & 1 & + & . & . & 1 & 1 \\
\hline Calystegia sepium & . & . & . & . & . & . & . & $\cdot$ & 1 & 1 & & $\cdot$ & . & + & 1 & + & . & + & . & . & 1 & . \\
\hline Valeriana dioica & . & . & + & . & 1 & + & . & . & . & . & + & & + & . & . & . & . & + & . & 1 & & . \\
\hline Eupatorium cannabinum & + & . & . & . & . & . & + & . & $\cdot$ & . & . & . & . & + & + & + & . & 1 & . & . & 1 & . \\
\hline Pentaglottis sempervirens & . & . & $r$ & 1 & . & . & . & + & . & + & + & . & . & . & + & . & . & . & + & . & . & . \\
\hline Agrostis capillaris & + & . & . & . & . & . & . & $\cdot$ & 1 & + & . & $\cdot$ & . & . & . & 1 & . & 1 & . & . & 1 & . \\
\hline Prunella vulgaris & . & + & . & . & . & . & . & . & + & 1 & $\cdot$ & . & . & + & . & . & . & 1 & + & . & . & . \\
\hline Senecio aquaticus & . & . & . & . & + & . & . & $\cdot$ & $\cdot$ & . & + & & + & . & . & . & . & + & + & . & + & . \\
\hline Limniris pseudacorus & + & . & . & . & . & . & . & $\cdot$ & + & + & $\cdot$ & . & + & . & . & . & + & . & $\mathrm{r}$ & . & . & . \\
\hline Rumex acetosa & . & . & . & . & $r$ & + & . & $\cdot$ & . & . & $\cdot$ & . & + & + & + & . & . & . & + & . & . & . \\
\hline Veronica chamaedrys & 1 & . & . & . & . & . & . & 1 & . & 1 & $\cdot$ & . & . & . & . & . & . & 1 & . & . & 1 & . \\
\hline Galium aparine & . & . & . & 1 & . & . & . & . & . & . & . & & . & . & . & 1 & . & . & + & + & + & . \\
\hline Centaurea rivularis & . & + & . & . & . & . & + & . & $\cdot$ & . & $\cdot$ & . & . & + & + & . & . & + & . & . & . & . \\
\hline Carex Iusitanica & . & . & . & . & . & . & . & + & $\cdot$ & $r$ & . & + & + & $\cdot$ & $\cdot$ & . & $\cdot$ & $\cdot$ & . & + & $\cdot$ & . \\
\hline Tritonia x crocosmiflora & . & . & . & . & . & . & . & . & $\cdot$ & . & . & $\cdot$ & . & $\cdot$ & 1 & . & 1 & + & . & 1 & . & . \\
\hline Lythrum salicaria & . & . & . & $r$ & . & ${ }^{\circ}$ & . & . & $\cdot$ & . & . & 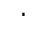 & . & . & . & . & $r$ & 1 & . & . & 1 & . \\
\hline Lycopus europaeus & + & . & . & . & . & 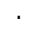 & . & 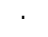 & + & . & 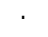 & . & . & . & . & . & 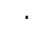 & + & . & . & + & . \\
\hline Carex pilulifera & . & . & . & . & + & . & . & 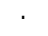 & $\cdot$ & . & + & + & . & . & . & . & . & + & . & . & . & . \\
\hline
\end{tabular}


Taxones presentes en menos de cuatro inventarios:

$\mathrm{E}_{1}+\mathrm{E}_{2}$ : Cytisus scoparius: + en 7 y r en 18; Malus sylvestris: + en 5; Prunus laurocerasus: + en 5 y r en 6; Prunus spinosa: 1 en 6, 1 en 13 y 1 en 16; Quercus pyrenaica: 1 en 6 y 1 en 21; Rosa micrantha: + en 5 y + en 17; Rosa sp.: + en 13 y + en 16; Viburnum opulus: 1 en 18. Taxones característicos de asociación y unidades superiores: Allium scorzonerifolium: + en 13, 1 en 16 y 2 en 17; Humulus lupulus: 1 en 16, + en 17 y + en 21. Taxones característicos de Quercetea robori-petraeae y Carpino-Fagetea: Doronicum plantagineum: + en 2; Dryopteris filix-mas: + en 5; Lathyrus linifolius: $r$ en 18; Melampyrum pratense: + en 5 y 1 en 7; Melittis melissophyllum: + en 5; Phyllitis scolopendrium: $r$ en 17, + en 19 y + en 22. Otros taxones: Anthoxanthum amarum: + en 6 y + en 8; Anthoxanthum odoratum:1 en 5; Apium nodiflorum: + en 21; Arrhenatherum bulbosum: $r$ en 9; Asphodelus lusitanicus: + en 5 y + en 15; Asplenium trichomanes: + en 4; Bellis perennis: $r$ en 19; Bidens sp.: + en 16; Bidens tripartita:1 en 18 y 1 en 21; Cardamine flexuosa: 1 en 1, + en 5 y 1 en 8; Carex sp.: + en 1 y + en 7; Chrysosplenium oppositifolium: + en 6, + en 12 y 1 en 22; Cirsium palustre: + en 4 y + en 14; Clematis vitalba: + en 22; Crocus serotinus: + en 17 y + en 19; Cystopteris sp.: + en 11; Epilobium sp.: + en 12, + en 18 y + en 21; Galium palustre: + en 5, 1 en 18 y + en 21; Galium sp.: + en 1 y + en 9; Glechoma hederacea: + en 8; Glyceria fluitans; + en 1; Hypericum tetrapterum: + en 16; Juncus effusus: + en 2; Lapsana communis: + en 4, + en 8 y 1 en 15; Lotus corniculatus: + en 18; Mentha aquatica: + en 5, + en 18 y + en 21; Molinia caerulea: 1 en 15; Narcissus bulbocodium: + en 1, 1 en 7 y + en 15; Petasites hybridus: 1 en 17; Phormium tenax: + en 20; Picris hieracioides: + en 11 y r en 19; Poa trivialis: + en 17; Polygonatum odoratum: 1 en 3; Polygonum persicaria: + en 10, + en 18 y 1 en 21; Polypodium cambricum: 1 en 15 y + en 22; Potentilla erecta:+ en 13, + en 14 y + en 18; Prunella grandiflora: + en 18; Rumex obtusifolius:+ en 21; Rumex sp.: + en 18; Ruscus aculeatus: + en 5 y + en 7; Scutellaria minor: + en 9; Selinum broteri: 1 en 18; Silene vulgaris: + en 2 y + en 18; Sparganium emersum: + en 21; Stellaria media: + en 4; Taraxacum gr. officinale: r en 19; Torilis sp.: 1 en 16; Urtica dioica: 1 en 16, + en 19 y 1 en 21; Vicia sepium: + en 16 y + en 17; Wahlenbergia hederacea: + en 18.

Procedencia de los inventarios:

1: C: Arzúa, Ribadiso, margen derecha delo Río Iso, aguas abajo del puente (570/4752);2: C: Coirós, Boqueixón, Río das Fervenzas (572/4785); 3: C: Arzúa, entre Boente y Castañeda, Río Boente (574/4752); 4: Po: Lalín, Catasós, Río Asneiro, aguas arriba de A Ponte Vella (575/4718); 5: C: Sobrado dos Monxes, Folgoso, O Sisto, Río Tambre (576/4767); 6: C: Sobrado dos Monxes, A Louseira, Río Tambre (577/4764); 7: C: Aranga, margen izquierda del Río Mandeo, aguas arriba de la Central Eléctrica da Ruxidoira (580/4786); 8: Lu: Palas de Rei, Sáa, margen izquierda del Río Pambre (588/4749); 9: Lu: Guntín, Río Ferreira, aguas arriba de Guntín, margen izquierda (595/4749); 10: Lu: Guntín, Río, Rego de Samai (606/4751); 11: Lu: Palas de Rei, Maceda, A Devesa Nova, Rego de Ferreira, aguas arriba de Ponte de Gándaras (590/4751); 12: Lu: Palas de Rei, entre Merlán y Ulloa, Rego de Chancela (592/4753); 13: C: Touro, Fontes Rosas, Rego das Pucheiras (552/4745); 14: C: Coristanco, San Paio, entre A Cuca y Castrobó, Río Calvar (516/4783); 15: C: Rianxo, marxe esquerda do Río Té, entre O Araño y Buía (518/4727); 16: C: Coristanco, A Miñata, Os Prados, Río Grande, aguas abajo de Ponte Lubiáns (523/4782); 17: C: Carballo, Ardaña, Noví, margen derecha del Río Grande (524/4781); 18: C: Santiago de Compostela, Ponte Albar, margen derecha del Río Tambre (535/4756); 19: C: A Laracha, entre Os Muíños de Abaixo y Centulle, Río Anllóns (536/4787); 20: C: A Laracha, Soandrés, Sta. Baia, Río Grande (542/4786); 21: C: Oroso, entre la Estación de FF.CC. y Sigüeiro, margen derecha del Río Tambre (543/4758); 22: C: Carral, entre A Cabra y Lale, Río Barcés, aguas abajo de su unión con el Rego da Pena Grande (546/4781). 
Tabla Ilc. Alisedas galaico-portuguesas (Senecioni bayonnensis-Alnetum glutinosae) variante típica, subvariante mesotrofa, facies típica (cont.)

\begin{tabular}{|c|c|c|c|c|c|c|c|c|c|c|c|c|c|c|c|c|c|c|c|c|c|c|}
\hline Pendiente $\left({ }^{\circ}\right)$ & $<2$ & 2 & 3 & 2 & $<2$ & $<2$ & $<2$ & 2 & $<2$ & 2 & 4 & 2 & $<2$ & 2 & 2 & $<2$ & $<2$ & 2 & 4 & 2 & 2 & 2 \\
\hline Orientación & $S$ & $S$ & SE & S & $\mathrm{N}$ & S & NW & NNE & NW & W & $\mathrm{N}$ & SW & SE & SE & SE & W & SW & SW & S & SSE & SSE & W \\
\hline Altura de copas (m) & 16 & 12 & 18 & 20 & 20 & 18 & 14 & 16 & 16 & 14 & 26 & 16 & 12 & 16 & 22 & 20 & 14 & 20 & $12-24$ & 24 & 15 & 18 \\
\hline Cob. $E_{1}(\%)$ & 100 & 100 & 100 & 100 & 95 & 90 & 100 & 100 & 95 & 100 & 95 & 95 & 90 & 100 & 90 & 90 & 90 & 100 & 100 & 100 & 90 & 100 \\
\hline Cob. $E_{2}(\%)$ & 20 & 50 & 20 & 30 & 20 & 30 & 20 & 25 & 20 & 15 & 30 & 10 & 25 & 40 & 20 & 30 & 40 & 80 & 20 & 45 & 30 & 20 \\
\hline Cob. $E_{3}(\%)$ & 95 & 100 & 95 & 90 & 90 & 90 & 100 & 95 & 100 & 95 & 65 & 100 & 95 & 80 & 95 & 95 & 100 & 90 & 85 & 85 & 70 & 95 \\
\hline Área $\left(m^{2}\right)$ & 300 & 200 & 300 & 500 & 400 & 400 & 300 & 400 & 300 & 300 & 600 & 300 & 500 & 300 & 300 & 500 & 500 & 500 & 600 & 500 & 500 & 500 \\
\hline $\mathrm{N}^{0}$ de taxones & 54 & 57 & 53 & 68 & 57 & 51 & 66 & 57 & 59 & 55 & 54 & 47 & 43 & 50 & 64 & 56 & 51 & 57 & 50 & 55 & 81 & 69 \\
\hline
\end{tabular}

\section{$\mathrm{E}_{1}(>4,0 \mathrm{~m})+\mathrm{E}_{2}(>1,5-4,0 \mathrm{~m}):$}

Alnus glutinosa

Quercus robur

Salix atrocinerea

Frangula alnus

Corylus avellana

Sambucus nigra

Laurus nobilis

Castanea sativa

Crataegus monogyna

Rosa gr. canina

Prunus avium

Acer pseudoplatanus

Ligustrum ovalifolium

Ilex aquifolium

Rosa micrantha

\section{$\mathrm{E}_{3}(<1,5 \mathrm{~m}):$}

Taxones diferenciales frente a Valeriano-Alnetum e Hyperico-Alnetum

\section{Fraxinus angustifolia}

Narcissus cyclamineus

Faxinus excelsior $x$ F. angustifolia

Anemone albida

Galium broterianum

Ulmus minor

\begin{tabular}{|c|c|c|c|c|c|c|c|c|c|c|c|c|c|c|c|c|c|c|c|c|c|}
\hline 5 & 5 & 4 & 4 & 4 & 5 & 4 & 4 & 4 & 5 & 4 & 5 & 5 & 4 & 5 & 3 & 5 & 4 & 5 & 4 & 4 & 5 \\
\hline 2 & $r$ & 1 & 2 & 1 & 1 & 1 & + & + & 1 & 1 & + & 1 & + & + & $r$ & + & + & 1 & 1 & 1 & 1 \\
\hline 1 & 2 & 1 & 2 & 1 & 2 & . & 1 & 2 & 2 & 1 & 2 & 1 & 1 & 1 & 3 & 1 & 2 & 1 & 2 & 1 & 1 \\
\hline 1 & . & $\cdot$ & 1 & + & 1 & + & 1 & 1 & . & + & 1 & 2 & 1 & 1 & 1 & + & . & . & 1 & 2 & + \\
\hline . & . & 2 & 1 & 1 & . & . & 3 & $\cdot$ & 1 & . & + & . & 1 & 1 & . & . & 2 & 2 & 1 & 1 & 1 \\
\hline 1 & 1 & . & . & 1 & 1 & + & 1 & . & + & . & 1 & $\cdot$ & . & . & . & 2 & 2 & . & 1 & 1 & 1 \\
\hline+ & $\cdot$ & + & 1 & + & . & . & 1 & 2 & . & 2 & . & . & . & $\cdot$ & . & . & 2 & 1 & . & 1 & . \\
\hline+ & . & 1 & 1 & + & . & + & 1 & . & . & 1 & . & $\cdot$ & . & 1 & . & $\cdot$ & $\cdot$ & . & 1 & $r$ & . \\
\hline . & . & 1 & 2 & . & . & . & . & 1 & . & 1 & 1 & . & . & 1 & $r$ & 1 & . & . & . & 1 & . \\
\hline . & + & . & + & . & . & + & . & . & . & . & . & + & 1 & 1 & . & + & . & . & . & 1 & . \\
\hline . & . & + & + & . & . & 1 & . & . & . & + & $\cdot$ & . & . & $r$ & . & . & . & + & + & . & . \\
\hline . & . & . & . & . & . & $\cdot$ & . & . & . & + & . & $\cdot$ & . & . & + & . & . & 1 & 1 & + & + \\
\hline 1 & . & . & . & + & . & . & . & + & . & $\cdot$ & . & $\cdot$ & . & $\cdot$ & . & 1 & . & . & . & . & . \\
\hline . & . & . & . & . & 1 & . & . & $\cdot$ & 1 & . & . & . & . & + & . & . & . & . & $r$ & . & . \\
\hline . & . & . & . & . & . & . & . & . & . & + & . & . & . & . & . & . & . & . & + & + & $r$ \\
\hline
\end{tabular}

Taxones diferenciales frente a Hyperico-Alnetum

Hedera hibernica

Viola riviniana

Omphalodes nitida

Viola palustris

Osmunda regalis

Carex reuteriana

Betula pubescens

Deschampsia subtriflora

Caltha palustris

Pyrus cordata

Carex laevigata

Angelica major

Peucedanum lancifolium

Saxifraga spathularis

Erica arborea

Aquilegia dichroa

Solidago virgaurea

Conopodium majus

Hieracium umbellatum

Narcissus triandrus

Narcissus asturiensis

Ceratocapnos claviculata

Physospermum cornubiense

Arbutus unedo

\section{Taxones diferenciales de subvariante mesotrofa}

Fraxinus excelsior

Lysimachia nemorum

Stachys sylvatica

Veronica montana

Potentilla montana

Mercurialis perennis

Ornithogalum pyrenaicum

Carex sylvatica

Melica uniflora 


\begin{tabular}{|c|c|c|c|c|c|c|c|c|c|c|c|c|c|c|c|c|c|c|c|c|c|c|}
\hline $\mathrm{N}^{0}$ de orden & 1 & 2 & 3 & 4 & 5 & 6 & 7 & 8 & 9 & 10 & 11 & 12 & 13 & 14 & 15 & 16 & 17 & 18 & 19 & 20 & 21 & 22 \\
\hline \multicolumn{23}{|c|}{ Taxones característicos de asociación y unidades superiores } \\
\hline Athyrium filix-femina & + & 2 & 2 & 2 & 1 & 2 & 3 & 1 & 1 & 1 & + & 2 & 3 & 4 & 2 & + & 2 & 3 & 2 & 1 & 1 & 1 \\
\hline Lonicera hispanica & 1 & 1 & 2 & 1 & + & 1 & 2 & 2 & 1 & 2 & 1 & 1 & 1 & 1 & 1 & . & 2 & 1 & 1 & 1 & 2 & 1 \\
\hline Primula acaulis & 1 & + & 1 & 1 & 1 & 1 & 1 & + & + & 1 & + & 1 & + & . & 1 & . & 2 & 1 & 1 & 1 & 1 & 1 \\
\hline Dryopteris affinis & 1 & 1 & 2 & . & 1 & 1 & 1 & 1 & 1 & . & + & 1 & 1 & + & 1 & $\cdot$ & . & + & + & 1 & 2 & 1 \\
\hline Blechnum spicant & 1 & . & 1 & 1 & 1 & + & + & + & + & + & $\cdot$ & 1 & 1 & 1 & 1 & $\cdot$ & $\cdot$ & + & + & + & + & + \\
\hline Euphorbia dulcis & 1 & + & 3 & 3 & 1 & 3 & 1 & . & 1 & 1 & + & 1 & $\cdot$ & . & + & . & . & 2 & 1 & 2 & 1 & 1 \\
\hline Ranunculus ficaria & 1 & · & 1 & + & 2 & 2 & · & 1 & 2 & 3 & 1 & 1 & $\cdot$ & . & 1 & $\cdot$ & $\cdot$ & 2 & 1 & 2 & $\cdot$ & 2 \\
\hline Senecio nemorensis & 1 & + & . & + & · & 3 & 3 & . & · & 2 & . & 3 & 2 & . & 1 & . & 2 & 2 & + & 2 & + & . \\
\hline Brachypodium sylvaticum & $\cdot$ & · & - & + & + & · & · & 1 & $\cdot$ & . & 1 & $\cdot$ & . & 2 & + & 1 & $\cdot$ & $\cdot$ & 1 & 1 & 1 & 1 \\
\hline Polystichum setiferum & · & . & . & 1 & 1 & . & . & 1 & 1 & . & 2 & + & . & . & 1 & . & $\cdot$ & $\cdot$ & 2 & . & 2 & + \\
\hline Carex remota & · & . & . & . & 1 & - & . & + & + & $\cdot$ & 1 & $\cdot$ & . & . & $\cdot$ & + & $\cdot$ & $r$ & 1 & $\cdot$ & + & 1 \\
\hline Hypericum androsaemum & $\cdot$ & . & . & + & 1 & . & + & + & + & + & 1 & . & . & 1 & $\cdot$ & . & . & . & + & $\cdot$ & . & . \\
\hline Myosotis martini & 1 & · & + & · & . & + & 1 & · & · & $\cdot$ & $\cdot$ & 2 & . & . & 1 & . & 1 & 1 & · & . & . & . \\
\hline Arum italicum & $\cdot$ & . & + & 1 & 1 & . & . & 1 & . & $\cdot$ & $\cdot$ & + & . & . & $\cdot$ & . & . & $\cdot$ & + & . & . & $r$ \\
\hline Luzula sylvatica & $\cdot$ & · & · & . & $\cdot$ & . & . & $\cdot$ & · & $\cdot$ & 1 & $\cdot$ & $\cdot$ & $\cdot$ & 2 & $\cdot$ & $\cdot$ & $\cdot$ & 1 & 3 & 1 & 2 \\
\hline Solanum dulcamara & . & . & . & . & . & + & + & . & · & . & $\cdot$ & . & . & + & . & 1 & 1 & $\cdot$ & $\cdot$ & $\cdot$ & . & . \\
\hline
\end{tabular}

Taxones característicos de Quercetea robori-petraeae y Carpino-Fagetea

Holcus mollis

Ajuga reptans

Polypodium vulgare

Teucrium scorodonia

Stellaria holostea

Potentilla sterilis

Hyacinthoides non-scripta

Euphorbia amygdaloides

Dryopteris dilatata

Oxalis acetosella

Crepis lampsanoides

Anemone nemorosa

Tamus communis

Aquilegia vulgaris

\section{Otros taxones}

Rubus sp.

Brachypodium rupestre

Heracleum sphondylium

Ranunculus repens

Geranium robertianum

Lamium maculatum

Dactylis glomerata

Rumex acetosa

Cardamine pratensis

Pteridum aquilinum

Eupatorium cannabinum

Pentaglottis sempervirens

Geum urbanum

Cardamine hirsuta

Galium aparine

Limniris pseudacorus

Prunella vulgaris

Lycopus europaeus

Calystegia sepium

Valeriana dioica

Scrophularia auriculata

Agrostis capillaris

Umbilicus rupestris

Urtica dioica

Senecio aquaticus

Angelica sylvestris

Polygonum persicaria

Galium palustre

Apium nodiflorum

Silene dioica

Cirsium palustre

Digitalis purpurea

Ruscus aculeatus 
Taxones presentes en menos de cuatro inventarios:

$\mathbf{E}_{\mathbf{1}}+\mathrm{E}_{\mathbf{2}}$ : Acacia dealbata: + en 16; Cytisus scoparius: $\mathrm{r}$ en 11; Cytisus striatus: $\mathrm{r}$ en 10 y + en 21; Malus domestica: + en 16; Prunus laurocerasus: 1 en 9; Prunus spinosa: + en 4; Quercus pyrenaica: 1 en 15; Robinia pseudoacacia: + en 4. Taxones característicos de asociación y unidades superiores: Carex pendula: 1 en 5, + en 11 y + en 14; Circaea lutetiana: 1 en 3, 1 en 19 y 1 en 21; Humulus lupulus: 2 en 2 y 2 en 16. Taxones característicos de Quercetea robori-petraeae y Carpino-Fagetea: Humulus lupulus: + en 11 y 1 en 22; Phyllitis scolopendrium: + en 8; Stachys officinalis: 1 en 4 y 1 en 7 . Otros taxones: Alliaria petiolata: + en 16; Anthoxanthum amarum: 1 en 9; Anthoxanthum odoratum: + en 21; Arrhenatherum bulbosum: 1 en 7, r en 13 y + en 14; Asphodelus lusitanicus: + en 4; Bidens sp.: + en 16 y + en 21; Bidens tripartita: + en 17; Bromus sterilis: + en 16; Bryonia dioica: 1 en 16 y + en 21; Cardamine flexuosa: + en 3 , + en 7 y + en 10; Carex binervis: + en 7; Carex cariophyllea: + en 4; Carex lusitanica: + en 10; Carex pilulifera: + en 15; Centaurea rivularis: + en 4 y r en 21; Chelidonium majus: + en 16 y + en 22; Chenopodium sp.: + en 16; Chrysosplenium oppositifolium: 1 en 5; Coincya setigera: + en 22; Crocus serotinus: + en 22; Epilobium sp.: + en 14; Festuca gr. rubra: + en 11 y + en 20; Festuca merinoi: + en 21; Filipendula ulmaria: 4 en 7 y 4 en 17; Fragaria vesca: + en 4; Fumaria sp.: r en 16; Galeopsis tetrahit: + en 7; Galium sp.: + en 13; Geranium lucidum: + en 22; Glechoma hederácea: + en 5; Glyceria fluitans: + en 2 y 1 en 18; Hieracium sp.: r en 11; Holcus lanatus: + en 10, 1 en 16 y r en 21; Hypericum sp.: r en 2 y r en 14; Hypericum tetrapterum: + en 14; Juncus effusus: + en 2 y + en 18; Lapsana communis: + en 16 y + en 18; Lysimachia vulgaris: 1 en 16; Lythrum salicaria: + en 4, 1 en 16 y + en 17; Mentha aquatica: 1 en 7 y 1 en 17; Mentha suaveolens: + en 14; Molinia caerulea: + en 11; Narcissus bulbocodium: + en 1 y + en 20; Oxalis corniculata: $r$ en 19; Oxalis pes-caprae: $r$ en 2; Oxalis sp.: r en 16; Phytolacca americana: 1 en 16; Picris hieracioides: r en 16; Poa annua: + en 10; Polygonatum odoratum: + en 12; Polygonum hydropiper: + en 14; Polygonum sp.: 2 en 16; Polypodium cambricum: 1 en 11 y + en 15; Potentilla erecta: 1 en 15; Rumex obtusifolius: + en 2, 1 en 16 y + en 18; Saponaria officinalis: + en 16 y + en 21; Saxifraga granulata: + en 22; Scutellaria minor: + en 13; Sonchus oleraceus: r en 2; Sparganium emersum: r en 18; Sparganium neglectum: 1 en 16; Spergularia media: + en 21 y + en 22; Taraxacum gr. officinale: + en 10, + en 12 y + en 22; Tradescantia fluminensis: 1 en 5; Trifolium repens: + en 11; Tritonia x crocosmiflora: + en 5 , + en 16 y + en 19; Veronica chamaedrys: 2 en 7, 1 en 15 y 1 en 17.

Procedencia de los inventarios:

1: C: Ordes, entre la villa y A Costa, Río Cabrón (549/4769); 2: C: O Pino, Amenal, Río de Amenal (549/4750); 3: C: Touro, entre Quintás y Vilar, Rego do Xermil (550/4742); 4: C: Touro, Quintás, O Castro, Rego de Brandelos (551/4745); 5: C: Carral, Rego da Brexa, aguas arriba del área recreativa (552/4787); 6: C: Touro, Muíña, Río das Lañas (559/4748); 7: C: Frades, praia fluvial de Ponte Carreira, margen derecha del Río Tambre (561/4763); 8: C: Oza-Cesuras, Paio, Río Mendo, aguas abajo de Ponte dos Cabalos (567/4786); 9: C: Monfero, Río do Vilariño, entre Abercovo y Sucamiño (570/4801); 10: C: Boimorto, Ribadiso de Mella (571/4760); 11: Ou: A Arnoia, entre O Rial ye Parbón, margen derecha del Río Arnoia (573/4675); 12: C: Melide, Barreiro, Barreiro do Medio, Río Catasol (578/4752); 13: Lu: Guntín, Ponte Zamai, Río Ferreira (605/4749); 14: Lu: Portomarín, Rego da Ferrería de Zamolle, entre Bagude y Belade (609/4739); 15: Lu: Guntín, entre Vilarmao y Casas Novas, Río Ferreira (611/4745); 16: Ou: Arnoia, Arnoia, Río Arnoia (571/4678); 17: C: Oroso, estación de FF.CC., Río Lengüelle, bosque en pequeña isla aguas arriba de su unión con el Río Tambre (543/4756); 18: C: O Pino, Arca, Río Brandelos (552/4751); 19: Po: Arbo, San Xoán, Río Deva, aguas arriba del puente de la N-540 (558/4663); 20: Ou: Avión, entre Pascais y Beresmo, margen derecha del Río Avia (562/4694); 21: Ou: Boborás, Pazos de Arenteiro, Río Avia (568/4694); 22: Ou: A Merca, Entrambosríos, A Medorra, Río Arnoia, margen izquierda (589/4671). 
Tabla III. Alisedas galaico-portuguesas (Senecioni bayonnensis-Alnetum glutinosae): 1-10: variante típica, subvariante mesotrofa, facies de contacto con alisedas cantábricas occidentales; 11-12: variante hiperoceánica, subvariante típica, facies típica; 13-14: variante hiperoceánica, subvariante mesotrofa, facies típica

\begin{tabular}{|c|c|c|c|c|c|c|c|c|c|c|c|c|c|c|}
\hline $\mathrm{N}^{0}$ de orden & 1 & 2 & 3 & 4 & 5 & 6 & 7 & 8 & 9 & 10 & 11 & 12 & 13 & 14 \\
\hline Altitud (m) & 20 & 10 & 185 & 250 & 35 & 225 & 205 & 15 & 290 & 260 & 60 & 125 & 115 & 280 \\
\hline Pendiente $\left({ }^{\circ}\right)$ & 2 & 2 & $<2$ & 2 & 2 & 2 & 4 & $<2$ & 4 & $<2$ & 4 & 10 & 10 & 6 \\
\hline Orientación & NW & NW & $\mathrm{N}$ & S & WNW & W & $\mathrm{NE}$ & W & SW & $\mathrm{N}$ & SW & S & $\mathrm{E}$ & W \\
\hline Altura de copas (m) & 16 & 18 & 14 & 18 & 12 & 20 & 28 & 28 & 17 & 18 & 20 & 12 & 20 & $8-16$ \\
\hline Cob. $E_{1}(\%)$ & 95 & 95 & 90 & 95 & 90 & 100 & 90 & 95 & 100 & 95 & 100 & 75 & 85 & 80 \\
\hline Cob. $E_{2}(\%)$ & 20 & 30 & 40 & 30 & 30 & 10 & 20 & 35 & 15 & 25 & 40 & 30 & 50 & 20 \\
\hline Cob. $E_{3}(\%)$ & 95 & 95 & 90 & 90 & 60 & 90 & 90 & 95 & 90 & 100 & 95 & 60 & 75 & 95 \\
\hline Área $\left(\mathrm{m}^{2}\right)$ & 400 & 500 & 400 & 300 & 200 & 200 & 300 & 500 & 450 & 300 & 300 & 300 & 300 & 240 \\
\hline $\mathrm{N}^{0}$ de taxones & 65 & 81 & 60 & 66 & 37 & 53 & 58 & 80 & 78 & 61 & 49 & 44 & 41 & 46 \\
\hline
\end{tabular}

\begin{tabular}{|c|c|c|c|c|c|c|c|c|c|c|c|c|c|c|}
\hline \multicolumn{15}{|c|}{$E_{1}(>4,0 \mathrm{~m})+E_{2}(>1,5-4,0 \mathrm{~m}):$} \\
\hline Alnus glutinosa & 5 & 4 & 4 & 4 & 3 & 5 & 3 & 3 & 4 & 5 & 4 & 3 & 3 & 4 \\
\hline Salix atrocinerea & 1 & 1 & 2 & + & . & 1 & 1 & 1 & 1 & 1 & 1 & 2 & + & 2 \\
\hline Corylus avellana & 2 & 3 & 3 & + & 1 & 1 & 1 & 2 & 2 & + & 3 & . & . & 1 \\
\hline Laurus nobilis & 1 & + & 1 & 1 & 1 & 1 & 1 & 1 & . & . & 1 & 2 & 2 & + \\
\hline Quercus robur & 1 & 1 & + & 1 & + & $\cdot$ & + & 1 & 1 & + & 1 & ${ }^{\circ}$ & 2 & 1 \\
\hline Crataegus monogyna & + & 1 & . & + & . & + & . & + & 1 & 1 & . & + & . & . \\
\hline Frangula alnus & $\cdot$ & + & . & + & $\cdot$ & - & . & + & · & + & + & + & 1 & + \\
\hline Sambucus nigra & . & + & 2 & 1 & . & + & + & 1 & + & . & . & . & . & . \\
\hline Castanea sativa & 1 & 1 & . & . & . & + & . & 1 & + & + & . & $\cdot$ & $\cdot$ & . \\
\hline Ilex aquifolium & $\cdot$ & . & . & . & . & . & + & . & + & + & 1 & . & . & 1 \\
\hline Ligustrum ovalifolium & + & . & . & . & . & 1 & + & + & . & . & . & . & . & . \\
\hline Acacia melanoxylon & . & . & . & . & . & . & . & . & . & . & . & 2 & 1 & $r$ \\
\hline Acer pseudoplatanus & 1 & 1 & . & + & . & . & . & . & . & . & . & . & . & . \\
\hline Cytisus scoparius & . & . & . & . & . & - & . & + & - & . & - & 1 & - & + \\
\hline Prunus avium & . & . & . & . & . & - & . & 1 & . & . & - & + & 1 & \\
\hline
\end{tabular}

$\mathrm{E}_{3}(<1,5 \mathrm{~m})$

Taxones diferenciales frente a Valeriano-Alnetum e Hyperico-Alnetum

\begin{tabular}{|c|c|c|c|c|c|c|c|c|c|c|c|c|c|c|}
\hline Fraxinus angustifolia & 1 & + & 2 & 2 & 3 & $\cdot$ & $\cdot$ & $\cdot$ & $\cdot$ & $\cdot$ & 2 & 1 & 3 & 2 \\
\hline Narcissus cyclamineus & . & 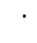 & ${ }^{\circ}$ & 1 & + & $\cdot$ & $\cdot$ & · & + & 1 & · & $\cdot$ & $\cdot$ & + \\
\hline \multicolumn{15}{|c|}{ Taxones diferenciales frente a Hyperico-Alnetum } \\
\hline Hedera hibernica & 2 & 2 & 2 & 2 & 1 & 5 & 2 & 2 & 1 & 3 & 2 & 2 & 2 & 1 \\
\hline Osmunda regalis & 2 & 1 & ${ }^{\prime}$ & 1 & 3 & . & + & 1 & + & 3 & 3 & 1 & 2 & 2 \\
\hline Carex reuteriana & + & 1 & . & 2 & 1 & . & + & 1 & 1 & + & 2 & + & 3 & 2 \\
\hline Omphalodes nitida & 1 & + & . & + & . & + & + & 1 & 1 & 1 & + & . & + & + \\
\hline Viola palustris & . & + & . & 1 & 1 & + & . & . & 1 & 1 & 2 & 1 & 3 & 2 \\
\hline Saxifraga spathularis & 1 & + & . & + & + & . & . & . & 2 & + & . & + & + & 1 \\
\hline Deschampsia subtriflora & + & + & . & 1 & ${ }^{\circ}$ & . & . & . & + & + & 1 & 1 & 1 & 1 \\
\hline Angelica major & $\mathrm{r}$ & + & . & + & . & . & + & + & $r$ & . & . & + & . & . \\
\hline Pyrus cordata & . & + & . & + & . & . & . & . & . & 1 & . & . & 1 & + \\
\hline Narcissus asturiensis & . & + & + & + & . & . & . & . & 1 & + & . & . & . & ${ }^{\circ}$ \\
\hline Caltha palustris & + & + & . & . & . & + & . & . & + & . & . & . & . & . \\
\hline Betula pubescens & · & 1 & . & . & . & . & . & . & 1 & 1 & . & . & . & . \\
\hline Erica arborea & $\cdot$ & . & . & . & . & . & . & . & + & . & . & + & . & 1 \\
\hline Carex laevigata & . & + & + & . & . & . & + & . & . & . & . & . & . & . \\
\hline Aquilegia dichroa & . & . & . & . & . & . & . & + & + & . & . & . & + & . \\
\hline Narcissus triandrus & + & . & . & . & + & . & . & . & . & . & . & $r$ & . & . \\
\hline Solidago virgaurea & . & . & . & . & + & . & . & . & . & . & . & 1 & . & . \\
\hline Euphorbia hyberna & . & . & . & + & . & . & . & . & + & . & . & . & . & . \\
\hline Hieracium umbellatum & . & . & . & . & + & . & . & . & . & . & + & . & . & . \\
\hline Allium scorzonerifolium & + & . & . & . & . & . & . & . & . & . & . & . & . & . \\
\hline Physospermum cornubiense & 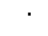 & . & . & . & . & . & . & . & . & + & . & . & . & . \\
\hline Conopodium majus & . & . & . & . & . & . & . & . & . & . & . & + & . & . \\
\hline
\end{tabular}

Taxa diferenciales de variante subhiperoceánica

Davallia canariensis

Dryopteris guanchica

Woodwardia radicans 


\begin{tabular}{|c|c|c|c|c|c|c|c|c|c|c|c|c|c|c|}
\hline $\mathrm{N}^{\circ}$ de orden & 1 & 2 & 3 & 4 & 5 & 6 & 7 & 8 & 9 & 10 & 11 & 12 & 13 & 14 \\
\hline \multicolumn{15}{|c|}{ Taxones diferenciales de subvariante mesotrofa } \\
\hline Fraxinus excelsior & 1 & 1 & 2 & 1 & . & 2 & 3 & 4 & 2 & 1 & . & & 1 & \\
\hline Lysimachia nemorum & + & 1 & + & . & + & + & + & 1 & 1 & $\cdot$ & . & & . & . \\
\hline Ranunculus tuberosus & 1 & + & . & . & $r$ & . & . & . & . & + & . & & . & + \\
\hline Mercurialis perennis & + & . & . & . & . & 2 & . & . & 1 & 1 & . & & . & . \\
\hline Veronica montana & . & . & + & . & . & . & 1 & 1 & + & . & . & . & . & \\
\hline Stachys sylvatica & . & + & . & . & . & . & + & + & . & . & . & & . & \\
\hline Carex sylvatica & . & + & . & . & . & . & . & + & . & . & . & . & . & \\
\hline Moehringia trinervia & $r^{\prime}$ & . & . & . & . & . & . & + & . & . & . & & . & \\
\hline Melica uniflora & & . & . & . & $r$ & . & . & & . & . & . & & & \\
\hline
\end{tabular}

Taxones diferenciales de facies de contacto con alisedas de Valeriano-Alnetum

Festuca gigantea

Chaerophyllum hirsutum

\section{Taxones característicos de asociación y unidades superiores}

Athyrium filix-femina

Viola riviniana

Lonicera hispanica

Euphorbia dulcis

Dryopteris affinis

Blechnum spicant

Ranunculus ficaria

Primula acaulis

Polystichum setiferum

Senecio nemorensis

Oxalis acetosella

Hypericum androsaemum

Brachypodium sylvaticum

Carex pendula

Carex remota

\begin{tabular}{|c|c|c|c|c|c|c|c|c|c|c|c|c|c|}
\hline 1 & 1 & 1 & 2 & 1 & 3 & 1 & 2 & 3 & 3 & 3 & 1 & 2 & 2 \\
\hline 1 & 1 & + & 1 & + & + & 1 & 1 & 1 & 1 & 1 & + & 1 & + \\
\hline 1 & + & 2 & 2 & . & 1 & 1 & 2 & 1 & 1 & + & + & 1 & 1 \\
\hline 1 & 1 & + & + & 1 & . & 1 & 1 & 2 & 1 & 1 & + & 1 & 1 \\
\hline 1 & 2 & 2 & 1 & . & 2 & 2 & 2 & 1 & 2 & + & + & 1 & . \\
\hline 1 & + & + & + & . & 1 & . & + & 1 & 1 & 1 & + & 1 & 1 \\
\hline 1 & 1 & 3 & 1 & . & + & 1 & + & 1 & 1 & + & . & . & . \\
\hline 1 & 1 & + & 1 & + & + & . & 1 & 1 & 1 & 1 & . & $\cdot$ & . \\
\hline . & 1 & 1 & + & 1 & 1 & 1 & 2 & 1 & . & + & . & . & . \\
\hline+ & 2 & 1 & 1 & . & . & + & 1 & 1 & 1 & + & . & . & . \\
\hline 1 & 1 & 1 & + & . & 1 & + & 1 & 1 & 1 & $\cdot$ & . & . & . \\
\hline 1 & 1 & + & + & . & + & + & + & + & . & . & . & + & . \\
\hline . & . & 1 & + & + & 1 & 1 & 2 & + & + & . & . & . & . \\
\hline+ & + & + & . & . & + & . & + & . & . & . & . & . & . \\
\hline+ & . & + & . & . & . & + & + & + & . & . & . & . & . \\
\hline
\end{tabular}

Taxones característicos de Quercetea robori petraeae y Carpino Fagetea

Dryopteris dilatata

Holcus mollis

Ajuga reptans

Teucrium scorodonia

Polypodium vulgare

Anemone nemorosa

Luzula sylvatica

Euphorbia amygdaloides

Crepis lampsanoides

Stellaria holostea

Hyacinthoides non-scripta

Aquilegia vulgaris

Potentilla sterilis

Stachys officinalis

\begin{tabular}{|c|c|c|c|c|c|c|c|c|c|c|c|c|c|}
\hline 1 & 2 & 1 & . & 1 & 1 & 2 & 1 & + & 1 & + & . & . & 1 \\
\hline 1 & + & 1 & . & . & 1 & + & + & 1 & + & + & 1 & · & 1 \\
\hline 1 & + & $r$ & 1 & + & + & + & 1 & 1 & 1 & 1 & . & . & . \\
\hline+ & + & . & 1 & . & . & + & 1 & + & 1 & + & + & + & + \\
\hline+ & + & + & . & + & + & + & + & + & 1 & + & · & . & 1 \\
\hline 1 & + & . & . & + & . & + & 1 & 1 & + & + & + & . & 1 \\
\hline 1 & 1 & . & 1 & 1 & $\cdot$ & . & · & 1 & 1 & 2 & 2 & . & . \\
\hline . & + & + & + & . & . & + & + & + & 1 & . & . & . & 1 \\
\hline+ & + & . & + & + & . & . & + & 1 & + & . & + & . & . \\
\hline+ & + & 1 & . & . & 1 & + & 1 & + & . & . & . & . & . \\
\hline 1 & 1 & + & . & . & . & + & 1 & + & . & . & + & . & . \\
\hline 1 & + & $\cdot$ & + & + & 1 & . & . & . & + & . & . & . & . \\
\hline+ & + & + & . & . & + & . & + & + & $\cdot$ & . & . & . & . \\
\hline 1 & 1 & . & 1 & + & . & . & . & . & . & · & . & . & . \\
\hline
\end{tabular}

\begin{tabular}{|c|c|c|c|c|c|c|c|c|c|c|c|c|c|c|}
\hline \multicolumn{15}{|l|}{ Otros taxones } \\
\hline Brachypodium rupestre & 2 & 2 & 1 & 2 & 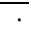 & . & 2 & 2 & 1 & 3 & + & 1 & 1 & 2 \\
\hline Rubus sp. & 1 & + & 1 & 1 & . & + & 1 & 1 & 1 & + & + & . & + & 1 \\
\hline Oenanthe crocata & 1 & 1 & + & 1 & 1 & + & + & 1 & 1 & $\cdot$ & + & $\cdot$ & . & . \\
\hline Geranium robertianum & . & + & + & + & . & + & + & 1 & + & . & + & . & + & . \\
\hline Heracleum sphondylium & . & 1 & 1 & + & . & + & + & 1 & + & 1 & . & . & . & . \\
\hline Lamium maculatum & . & 1 & 1 & 1 & . & + & 1 & + & + & + & . & . & . & . \\
\hline Pteridum aquilinum & . & . & $r$ & + & . & . & + & . & . & + & + & + & 1 & + \\
\hline Dactylis glomerata & + & + & + & + & . & . & · & . & . & + & + & . & + & + \\
\hline Silene dioica & + & 1 & 1 & + & . & . & 1 & 1 & 1 & . & . & . & . & . \\
\hline Angelica sylvestris & . & . & 1 & + & . & + & . & . & + & 1 & + & . & . & . \\
\hline Umbilicus rupestris & + & 1 & . & . & . & + & $r$ & . & . & . & . & 1 & . & + \\
\hline Tritonia x crocosmiflora & . & . & + & + & . & . & + & . & . & $\cdot$ & + & 1 & + & . \\
\hline Rumex acetosa & + & + & $r$ & . & . & . & . & . & + & . & + & + & . & . \\
\hline Ruscus aculeatus & . & . & . & + & + & + & . & + & . & . & 1 & . & . & · \\
\hline Eupatorium cannabinum & $\cdot$ & . & . & + & . & . & . & + & + & + & + & . & . & . \\
\hline Cardamine hirsuta & . & + & + & · & . & . & + & + & $r$ & $\cdot$ & . & $\cdot$ & . & · \\
\hline Anthoxanthum amarum & 1 & 1 & + & . & . & + & . & . & . & . & . & . & . & . \\
\hline Cardamine pratensis & 1 & + & + & . & . & . & . & . & + & . & . & . & . & . \\
\hline Geum urbanum & + & + & . & - & . & . & + & 1 & $\cdot$ & . & . & $\cdot$ & . & · \\
\hline Veronica chamaedrys & $\cdot$ & . & + & + & . & . & . & 1 & + & $\cdot$ & . & . & . & . \\
\hline Centaurea rivularis & . & . & . & 1 & . & . & . & . & + & + & . & . & + & . \\
\hline Narcissus bulbocodium & $\cdot$ & . & . & 1 & . & . & . & . & . & + & . & + & . & + \\
\hline Prunella vulgaris & . & + & . & + & . & . & . & + & + & . & . & . & . & . \\
\hline Digitalis purpurea & . & + & . & . & + & . & . & . & $r$ & . & . & . & + & . \\
\hline
\end{tabular}


Taxones presentes en menos de cuatro inventarios:

$\mathrm{E}_{1}+\mathrm{E}_{2}$ : Juglans regia: $\mathrm{r}$ en 7; Philadelphus coronarius: + en 7; Rosa gr. canina: + en 1 y + en 5; Ulmus glabra: 2 en 5 y 1 en 9 . Taxones característicos de asociación y unidades superiores: Arum italicum: 1 en 3 , + en 8 y + en 9; Circaea lutetiana: 1 en 7 y 1 en 8 ; Humulus lupulus: + en 8; Myosotis martini: + en 8; Symphytum tuberosum: + en 6; Taxones característicos de Quercetea robori-petraeae y Carpino-Fagetea: Dryopteris aemula: + en 2 y + en 9; Hypericum pulchrum: + en 14; Phyllitis scolopendrium: 1 en 3 , 1 en 6 y + en 8; Tamus communis: + en 6, + en 8 y + en 9. Otros taxones: Agrostis capillaris: 1 en 13; Anthoxanthum odoratum: + en 9; Apium nodiflorum: + en 8 y r en 11; Arrhenatherum bulbosum: 1 en 1, + en 2 y + en 10; Asphodelus lusitanicus: + en 2 y + en 8; Asplenium billotii: r en 12; Asplenium trichomanes: + en 13; Calluna vulgaris: + en 12; Calystegia sepium: + en 8; Chrysosplenium oppositifolium: + en 1, + en 3 y + en 9; Cirsium palustre: + en 2, + en 3 y + en 9; Cruciata glabra: + en 4 y + en 10; Cystopteris fragilis: + en 6; Filipendula ulmaria: 2 en 4 y 1 en 10; Fragaria vesca: + en 6 y + en 13; Galium aparine: + en 3; Glechoma hederacea: 1 en 3, 1 en 6 y 1 en 8 ; Juncus effusus: r en 8, r en 11 y + en 13; Lapsana communis: $r$ en 6; Limniris pseudacorus: + en 1 y + en 8; Lycopus europaeus: + en 4 y + en 8; Lythrum salicaria: 1 en 4; Molinia caerulea: + en 11, 1 en 13 y 1 en 14; Pentaglottis sempervirens: + en 8; Peucedanum gallicum: + en 11; Picris hieracioides: + en 2 y + en 11; Polygonum sp.: + en 7; Polypodium cambricum: 1 en 6 y + en 12; Ranunculus repens: + en 3 , + en 7 y 1 en 10; Rumex obtusifolius: + en 2; Scrophularia auriculata: + en 2, + en 4 y + en 14; Senecio aquaticus: + en 14; Succisa pratensis: + en 10; Tradescantia fluminensis: + en 2, + en 12 y r en 13; Urtica dioica: + en 9; Valeriana dioica: + en 8, 1 en 9 y + en 10; Valeriana officinalis: + en 14 .

\section{Procedencia de los inventarios:}

1: C: Miño, Callobre, Río Lambre, margen derecha aguas abajo de Ponte da Ribeira (568/4796); 2: C: Paderne, Viñas, Río Lambre, aguas arriba de A Veiga (566/4797); 3: C: Oza dos Ríos, Burricios, margen derecha del Río Mendo, augas arriba del puente (567/4783); 4: C: Aranga/lrixoa, Ponte de Muniferral, Río Mandeo (576/4788); 5: C: Coirós, Couto de Chelo, margen izquierda del Río Mandeo, a la altura de la Capela da Espenuca (569/4790); 6: C: Oza dos Ríos, A Barra, Río Miñatos (571/4783); 7: C: Oza-Cesuras, Trasanquelos, Río Mendo, aguas abajo de A Ponte (567/4780); 8: C: Betanzos, Brabío, O Marulo, margen izquierda del Río Mendo, aguas abajo del puente (565/4790); 9: C: Aranga, O Barreiro, Río Cambás, aguas arriba de Ponte da Rosa Mala (580/4786); 10: C: Aranga, San Paio, Río Mandeo, aguas debajo de la unión con el Rego das Balsas (580/4786); 11: C: Boiro, Río Beluso, aguas abajo de A Cabaleiriza (514/4729); 12: C: Pobra do Caramiñal, Río das Pedras (504/4720); 13: C: Boiro, Cures, Barranqueira da Devesa da Graña (508/4727); 14: C: Lousame, Toxos Outos, Río de San Xusto (514/4739). 
Tabla IVa. Alisedas cantábricas occidentales (Valeriano pyrenaicae-Alnetum glutinosae) variante típica, subvariante típica

\begin{tabular}{|c|c|c|c|c|c|c|c|c|c|c|c|c|c|c|c|c|c|c|c|c|c|c|c|c|}
\hline $\mathrm{N}^{\circ}$ de orden & 1 & 2 & 3 & 4 & 5 & 6 & 7 & 8 & 9 & 10 & 11 & 12 & 13 & 14 & 15 & 16 & 17 & 18 & 19 & 20 & 21 & 22 & 23 & 24 \\
\hline Altitud (m) & 155 & 15 & 155 & 130 & 5 & 5 & 95 & 10 & 95 & 15 & 415 & 300 & 350 & 230 & 115 & 195 & 105 & 150 & 110 & 70 & 235 & 175 & 285 & 205 \\
\hline Pendiente $\left({ }^{\circ}\right)$ & 4 & $<2$ & 2 & 4 & 2 & 2 & 2 & $<2$ & 6 & $<2$ & 2 & 4 & 2 & 4 & 4 & 4 & 4 & 6 & 4 & 2 & $<2$ & 2 & 2 & $<2$ \\
\hline Orientación & $\mathrm{N}$ & $\mathrm{N}$ & ENE & ESE & NNW & ENE & NW & $\mathrm{N}$ & NNW & WSW & WNW & W & W & SW 1 & NW & SSW & $\mathrm{NE}$ & NW & NW & $\bar{N}$ & $\mathrm{NE}$ & SW & W & $\mathrm{NW}$ \\
\hline Altura de copas & 14 & 22 & 16 & 20 & 12 & 22 & 20 & 22 & 24 & 18 & 18 & 16 & 20 & 18 & 22 & 22 & 26 & 10 & 10 & 14 & 24 & 22 & 14 & 20 \\
\hline ob. $E_{1}(\%)$ & 90 & 100 & 100 & 95 & 100 & 100 & 100 & 100 & 100 & 95 & 100 & 95 & 100 & 95 & 100 & 100 & 100 & 85 & 100 & 100 & 100 & 100 & 100 & 100 \\
\hline ob. E & 30 & 20 & 20 & 15 & 10 & 10 & 15 & 20 & 20 & 20 & 10 & 40 & 30 & 30 & 50 & 50 & 45 & 30 & 20 & 40 & 20 & 30 & 50 & 20 \\
\hline & 70 & 95 & 90 & 70 & 95 & 90 & 85 & 95 & 85 & 95 & 100 & 95 & 90 & 80 & 90 & 90 & 90 & 85 & 70 & 90 & 100 & 95 & 65 & 90 \\
\hline Área $\left(m^{2}\right)$ & 300 & 300 & 300 & 200 & 150 & 200 & 400 & 300 & 300 & 400 & 300 & 400 & 400 & 300 & 400 & 300 & 500 & 300 & 300 & 300 & 300 & 300 & 300 & 400 \\
\hline$N^{0}$ de taxones & 53 & 44 & 36 & 53 & 44 & 39 & 64 & 52 & 39 & 60 & 38 & 62 & 41 & 51 & 67 & 72 & 83 & 45 & 67 & 48 & 54 & 52 & 42 & 36 \\
\hline \multicolumn{25}{|l|}{$E_{1}(>4,0 \mathrm{~m})+E_{2}(>1,5-4,0 \mathrm{~m}):$} \\
\hline Alnus glutinosa & 4 & 5 & 4 & 5 & 5 & 5 & 4 & 4 & 5 & 5 & 5 & 5 & 3 & 4 & 4 & 4 & 4 & 3 & 3 & 3 & 5 & 5 & 5 & 5 \\
\hline alix atrocinerea & + & 2 & 2 & + & 1 & + & 1 & 2 & + & + & 1 & 1 & + & 1 & 1 & + & 2 & 3 & 1 & 1 & 1 & 1 & 1 & \\
\hline aurus nobilis & 1 & 1 & + & + & 1 & 1 & 2 & + & 1 & 2 & 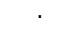 & . & . & 1 & 1 & 1 & 1 & 1 & + & 1 & 2 & 1 & + & + \\
\hline Ilana & 1 & 1 & . & 1 & . & + & 1 & 2 & 2 & 1 & . & 1 & 1 & 2 & 2 & 3 & 3 & 2 & 4 & 3 & $\cdot$ & + & 1 & 1 \\
\hline Fra & + & 1 & . & 1 & . & + & 2 & 1 & & 1 & + & + & . & 3 & 2 & 4 & 2 & 1 & 3 & 3 & 2 & 2 & 1 & . \\
\hline Cas & 2 & & . & + & + & 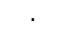 & & 1 & 1 & 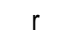 & + & + & + & $r$ & + & + & & 1 & & 1 & + & & 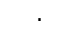 & + \\
\hline Samb & . & 1 & 2 & 1 & 1 & + & 1 & 1 & 2 & 1 & . & . & . & . & + & + & 1 & . & . & 1 & 1 & . & . & . \\
\hline Querc & 1 & 1 & . & . & . & 1 & . & . & 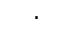 & + & 1 & 1 & 3 & $r$ & . & 2 & . & . & . & + & 1 & 1 & . & 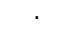 \\
\hline Crata & . & . & . & 1 & . & . & . & . & . & . & 1 & 1 & . & 1 & 1 & 1 & 1 & & 1 & 1 & . & . & 1 & + \\
\hline Acer & . & . & . & . & . & . & . & + & + & 1 & 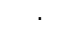 & . & 1 & 2 & 1 & 1 & 1 & . & . & . & . & . & 1 & 1 \\
\hline Frangula alnu & + & . & . & 1 & . & . & . & + & . & . & 1 & + & 1 & 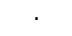 & + & . & . & . & . & + & . & . & 1 & + \\
\hline ovalifoliur & . & 1 & + & . & 1 & 1 & . & 2 & . & . & . & . & . & . & . & . & + & . & . & . & 1 & . & 1 & . \\
\hline & + & . & . & . & . & . & + & . & . & . & + & . & + & . & . & . & . & . & . & . & + & 1 & . & ${ }^{\circ}$ \\
\hline olium & + & . & . & + & . & . & . & . & . & . & . & + & . & . & + & . & . & 1 & . & . & . & . & . & + \\
\hline Pyrus cordata & $\cdot$ & . & . & . & . & . & . & . & . & . & . & 1 & 1 & . & 1 & . & - & & . & + & . & . & - & 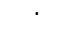 \\
\hline \multicolumn{25}{|c|}{$\begin{array}{l}E_{3}(<1,5 \mathrm{~m}): \\
\text { Taxones diferenciales frente a Senecioni-Alnetum }\end{array}$} \\
\hline Chaerophyllum hirsutum & + & + & 3 & 1 & + & 1 & + & & 2 & + & & & & 1 & & + & 1 & 1 & + & 1 & 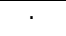 & & & \\
\hline Vale & . & + & 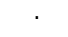 & 2 & + & . & 2 & . & 1 & + & . & . & . & 1 & + & 1 & . & . & . & . & . & 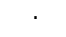 & 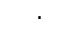 & \\
\hline Festu & . & . & . & 1 & + & . & . & . & . & + & . & 1 & . & $\cdot$ & . & + & + & . & . & + & . & + & . & 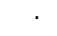 \\
\hline $\mathrm{rm}$ & . & . & + & . & . & . & . & . & . & . & $r$ & + & + & . & . & . & . & . & . & + & + & + & . & + \\
\hline Aver & + & . & 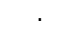 & . & . & . & + & . & 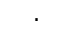 & . & . & + & . & . & . & . & . & 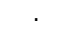 & & . & . & . & 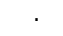 & + \\
\hline Scro & + & . & . & + & . & . & . & + & . & . & . & . & . & . & . & . & . & . & . & . & . & . & . & $\cdot$ \\
\hline Sax & + & . & . & . & . & . & 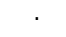 & + & 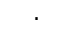 & . & . & $r$ & . & . & . & . & . & . & . & . & . & . & 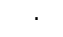 & . \\
\hline Adenos & . & . & . & . & . & . & . & . & . & . & . & . & . & . & + & . & . & . & + & . & . & . & 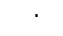 & $\cdot$ \\
\hline Caro & . & . & . & . & . & . & 1 & . & 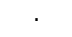 & 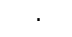 & . & . & . & . & . & . & . & . & . & . & . & . & 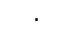 & \\
\hline Narci & . & . & . & . & 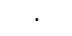 & . & + & . & & . & 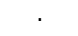 & . & . & . & . & . & . & . & . & . & . & . & . & . \\
\hline Cytisus commutatus & . & . & . & . & . & . & 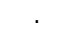 & . & & . & . & . & . & & . & . & . & . & . & . & . & . & + & . \\
\hline \multicolumn{25}{|c|}{ Taxones diferenciales frente a Hyperico-Alnetum } \\
\hline Hede & 2 & 2 & 3 & 2 & 3 & 4 & 2 & 2 & 3 & 3 & 3 & 3 & 3 & 2 & 2 & 2 & 2 & 1 & 2 & & 2 & 3 & 2 & 2 \\
\hline & 1 & + & . & 1 & . & . & + & . & . & . & . & 1 & 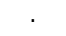 & 1 & 1 & + & + & . & 1 & + & 1 & + & 1 & 1 \\
\hline Vio & + & . & . & 1 & . & . & 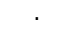 & + & . & . & 2 & 2 & 3 & . & + & 1 & . & . & + & 1 & 2 & 1 & 1 & 1 \\
\hline On & + & . & & . & . & . & & . & & . & 1 & 1 & & 1 & + & 1 & + & 1 & + & + & 1 & + & . & . \\
\hline trit & 1 & + & . & . & . & . & + & . & . & . & 1 & 1 & 2 & + & + & . & . & . & . & . & + & + & 3 & . \\
\hline & 1 & . & . & . & . & . & . & 1 & . & . & 1 & 1 & 1 & . & 1 & . & 2 & 1 & . & . & 1 & 1 & . & 1 \\
\hline & 1 & . & 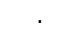 & . & . & . & . & . & & . & . & 1 & 1 & . & 1 & . & . & . & + & 1 & + & 1 & . & 2 \\
\hline Sol & 1 & . & 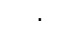 & + & . & . & . & . & & . & . & . & + & . & . & . & . & . & . & . & 1 & . & . & . \\
\hline arcissus asturiensi & . & . & . & . & . & . & . & . & 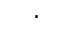 & . & . & . & . & . & . & . & . & . & 1 & . & 1 & + & . & . \\
\hline Euphorbia hyberna & . & . & . & . & 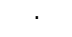 & 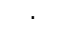 & & . & & . & & + & . & & 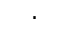 &. & . & & 1 & . & . & . & + & \\
\hline & . & . & . & . & . & . & . & . & & . & . & . & . & . & . & . & . & . & . & . & . & . & 1 & 1 \\
\hline & . & . & 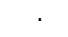 & . & 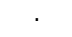 & . & & . & & . & . & . & . & . & + & . & . & . & . & . & . & . & + & \\
\hline & 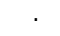 & . & . & . & & . & & . & & . & & + & . & & . & . & . & 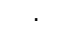 & . & . & . & . & . & \\
\hline Hieracium umbellatum & . & . & & 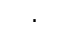 & & 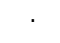 & & 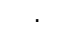 & & 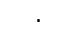 & & + & 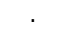 & & . & 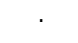 & . & & & . & . & & 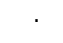 & \\
\hline \multicolumn{25}{|c|}{ ción y unidades superiores } \\
\hline & 1 & 1 & 3 & 3 & 3 & 2 & 1 & 3 & 4 & 2 & 2 & 3 & & 1 & & & 2 & 1 & 1 & 1 & 3 & 2 & & 2 \\
\hline & 1 & . & 1 & 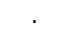 & + & 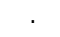 & 1 & 2 & + & 1 & 1 & 1 & 2 & 1 & 1 & 2 & + & 1 & 1 & + & 1 & 1 & 1 & 1 \\
\hline & + & + & + & 1 & 1 & 1 & + & 1 & 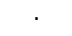 & 1 & 1 & . & + & . & 1 & + & 1 & 1 & 1 & 1 & 1 & 1 & 1 & 1 \\
\hline Oxalis acetosella & + & 1 & + & 2 & 1 & + & 2 & 1 & 2 & 1 & . & 1 & . & 2 & 2 & 2 & 2 & 1 & 2 & 1 & . & 1 & 1 & . \\
\hline Ble & + & + & 1 & + & . & . & 1 & + & & . & 1 & 1 & 1 & 1 & 1 & $T$ & + & 1 & 1 & 1 & 1 & 1 & 1 & 1 \\
\hline Osmunda regalis & 2 & 1 & 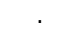 & 3 & 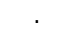 & 2 & + & + & & 2 & + & 4 & 4 & & 3 & 1 & + & 1 & 1 & 1 & 1 & 1 & . & 2 \\
\hline
\end{tabular}




\begin{tabular}{|c|c|c|c|c|c|c|c|c|c|c|c|c|c|c|c|c|c|c|c|c|c|c|c|c|}
\hline $\mathrm{N}^{0}$ de orden & 1 & 2 & 3 & 4 & 5 & 6 & 7 & 8 & 9 & 10 & 11 & 12 & 13 & 14 & 15 & 16 & 17 & 18 & 19 & 20 & 21 & 22 & 23 & 24 \\
\hline Dryopteris affinis & . & 1 & 1 & . & . & . & 1 & 1 & 2 & 1 & 2 & 2 & 1 & . & 1 & 2 & 1 & 1 & 1 & 1 & 1 & 2 & . & 1 \\
\hline Hypericum androsaemum & + & + & 1 & 1 & + & . & + & + & 1 & 2 & $\cdot$ & + & . & + & + & + & 1 & + & + & . & . & + & . & . \\
\hline Brachypodium sylvaticum & + & 1 & + & 1 & 1 & 1 & 1 & 1 & + & 2 & . & . & . & . & 1 & 1 & 1 & . & 1 & 1 & . & + & . & . \\
\hline Euphorbia dulcis & . & . & . & 1 & . & . & . & . & . & $\cdot$ & 1 & 1 & 1 & 2 & 2 & 2 & 2 & 1 & 2 & + & 1 & + & 1 & 1 \\
\hline Polystichum setiferum & . & . & 1 & . & 1 & 1 & 2 & . & . & 2 & . & + & . & . & 1 & 2 & 2 & 1 & 1 & + & . & . & . & . \\
\hline Senecio nemorensis & . & . & . & . & 1 & . & . & . & . & . & 1 & 1 & 1 & . & + & + & + & . & + & . & 1 & + & + & 1 \\
\hline Carex remota & . & . & + & + & . & 1 & 1 & . & + & $\cdot$ & . & . & . & + & + & + & + & 1 & + & + & . & . & . & . \\
\hline Primula acaulis & . & + & . & . & . & . & . & . & . & . & . & + & . & 1 & 1 & + & 1 & + & 1 & + & 1 & + & . & . \\
\hline Luzula sylvatica & . & 1 & . & 1 & . & . & + & . & + & . & . & 2 & 1 & . & + & . & . & . & + & . & + & . & + & . \\
\hline Lysimachia nemorum & . & . & + & + & . & . & 1 & . & . & 1 & . & . & . & + & + & + & 1 & . & . & + & . & . & + & . \\
\hline Carex pendula & . & . & - & + & 1 & 1 & 1 & . & 2 & 1 & . & . & . & . & + & . & 1 & . & . & + & . & . & . & . \\
\hline Circaea lutetiana & . & + & . & . & . & 1 & . & + & . & 1 & . & . & . & + & . & 2 & 1 & . & . & + & . & . & . & . \\
\hline Veronica montana & . & . & · & + & . & $\cdot$ & . & . & + & 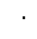 & $\cdot$ & . & · & 1 & + & 1 & 1 & . & + & $\cdot$ & . & . & . & · \\
\hline Arum italicum & . & . & . & . & . & + & 2 & . & . & + & . & . & . & + & . & + & + & . & + & . & . & . & . & . \\
\hline Mercurialis perennis & . & . & . & . & . & . & 2 & . & . & $\cdot$ & . & . & . & 1 & + & + & 3 & . & 1 & $\cdot$ & . & . & . & $\cdot$ \\
\hline Sanicula europaea & . & . & . & . & . & . & + & . & . & . & . & . & . & 1 & 1 & 1 & 2 & . & + & . & . & . & . & . \\
\hline Pulmonaria longifolia & . & . & . & . & . & + & . & . & . & . & . & . & . & $\cdot$ & 1 & . & 1 & . & + & . & . & + & . & . \\
\hline Myosotis martini & . & . & . & . & . & . & . & . & . & 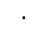 & . & . & . & . & . & 1 & 1 & + & + & . & . & . & . & . \\
\hline
\end{tabular}

Taxones característicos de Quercetea robori-petraeae y Carpino-Fagetea

\begin{tabular}{|c|c|c|c|c|c|c|c|c|c|c|c|c|c|c|c|c|c|c|c|c|c|c|c|c|}
\hline Dryopteris dilatata & 1 & 1 & 1 & 1 & 1 & . & + & 1 & 1 & 1 & $\cdot$ & 1 & $\cdot$ & + & 1 & 1 & 1 & 1 & + & 1 & 1 & 1 & + & + \\
\hline Ajuga reptans & 1 & . & + & 1 & + & . & + & + & 1 & + & . & 1 & $\cdot$ & 1 & 1 & 1 & 2 & + & + & 1 & + & 1 & 1 & 1 \\
\hline Holcus mollis & . & + & 1 & 1 & + & 1 & 1 & . & . & 1 & 1 & + & 1 & $\cdot$ & 1 & 1 & $\cdot$ & 1 & + & 1 & 1 & 2 & . & 1 \\
\hline Polypodium vulgare & . & + & + & + & + & . & + & + & + & + & . & 1 & 1 & $\cdot$ & + & 1 & 1 & + & + & $\cdot$ & + & + & . & . \\
\hline Teucrium scorodonia & 1 & + & . & . & + & . & . & + & $\cdot$ & + & 1 & + & 1 & $\cdot$ & $\cdot$ & . & $\cdot$ & + & . & . & 1 & 1 & + & 1 \\
\hline Aquilegia vulgaris & . & + & . & + & $\cdot$ & + & + & + & . & + & . & $\cdot$ & $\cdot$ & + & + & + & + & . & + & $\cdot$ & . & 1 & . & . \\
\hline Hyacinthoides non-scripta & . & + & . & . & · & $\cdot$ & 1 & + & · & . & . & $\cdot$ & $\cdot$ & + & + & + & + & · & 2 & · & 1 & 1 & 1 & · \\
\hline Crepis lampsanoides & 1 & 1 & . & 1 & · & · & + & 1 & · & · & . & + & $\cdot$ & $\cdot$ & $\cdot$ & + & + & · & + & $\cdot$ & 1 & . & . & · \\
\hline Phyllitis scolopendrium & . & . & + & . & . & + & 1 & . & + & + & . & $\cdot$ & $\cdot$ & + & $\cdot$ & + & 1 & . & + & + & . & . & . & . \\
\hline Anemone nemorosa & . & . & . & . & · & . & + & . & . & . & $\cdot$ & + & + & $\cdot$ & 2 & + & 2 & . & 2 & $\cdot$ & . & . & + & + \\
\hline Euphorbia amygdaloides & . & + & . & . & · & . & . & . & . & . & $\cdot$ & + & $\cdot$ & $\cdot$ & + & · & + & . & + & + & + & . & + & . \\
\hline Tamus communis & . & . & . & . & 1 & 1 & . & . & . & + & . & $\cdot$ & $\cdot$ & $\cdot$ & $\cdot$ & + & 1 & + & + & . & . & . & . & . \\
\hline Stachys officinalis & . & . & . & + & · & . & . & . & . & . & . & 1 & + & $\cdot$ & + & · & + & . & . & . & + & 1 & . & . \\
\hline Potentilla sterilis & . & . & . & . & + & . & 1 & . & . & . & . & $\cdot$ & $\cdot$ & 1 & $\cdot$ & + & + & . & . & + & . & . & . & . \\
\hline Stellaria holostea & . & . & . & . & · & · & . & . & . & . & $\cdot$ & $\cdot$ & $\cdot$ & 1 & $\cdot$ & 1 & 1 & . & + & . & + & . & . & . \\
\hline
\end{tabular}

\begin{tabular}{|c|c|c|c|c|c|c|c|c|c|c|c|c|c|c|c|c|c|c|c|c|c|c|c|c|}
\hline Otros taxones & & & & & & & & & & & & & & & & & & & & & & & & \\
\hline Rubus sp. & 1 & 2 & 2 & 1 & 1 & 1 & + & 1 & 2 & 1 & 2 & + & + & 1 & + & 1 & + & 1 & + & . & 1 & 1 & 1 & 1 \\
\hline Oenanthe crocata & + & 1 & 1 & + & 2 & 1 & 1 & 1 & 2 & + & 1 & 1 & 2 & + & 1 & 1 & + & 1 & + & . & 1 & + & + & 1 \\
\hline Brachypodium rupestre & . & $\cdot$ & . & . & $\cdot$ & $\cdot$ & . & 1 & . & 1 & 3 & 1 & 1 & 1 & + & 1 & 1 & 1 & + & . & 3 & 1 & 2 & 4 \\
\hline Angelica sylvestris & + & + & 2 & 1 & 1 & . & 1 & · & 1 & 1 & $\cdot$ & + & . & . & + & $\cdot$ & . & 1 & + & + & · & · & · & · \\
\hline Geranium robertianum & . & . & . & + & 1 & 1 & 1 & + & 1 & + & . & . & . & + & . & 1 & 1 & 1 & + & . & . & . & . & . \\
\hline Cardamine pratensis & + & . & + & 1 & . & . & . & . & 2 & . & + & . & . & 1 & + & + & + & + & . & . & + & . & . & . \\
\hline Lamium maculatum & . & 1 & + & . & 1 & . & 1 & 1 & + & 1 & 1 & . & . & . & . & + & + & . & . & . & 1 & . & · & . \\
\hline Caltha palustris & . & · & · & . & $\cdot$ & $\cdot$ & . & · & · & . & $\cdot$ & $r$ & + & + & + & + & + & 1 & + & · & · & + & 1 & 1 \\
\hline Scrophularia auriculata & + & + & $r$ & . & + & + & 1 & . & . & + & + & . & . & . & . & $\cdot$ & + & + & . & + & . & · & . & . \\
\hline Silene dioica & . & 1 & . & . & 1 & 1 & 1 & . & 3 & 2 & . & . & . & 1 & . & 1 & 1 & . & + & . & . & . & . & . \\
\hline Dactylis glomerata & + & 1 & + & . & + & . & . & 1 & . & + & 1 & + & . & . & . & . & + & . & . & . & + & . & . & . \\
\hline Pteridum aquilinum & + & . & . & . & $\cdot$ & . & . & . & . & . & + & . & + & . & + & $\cdot$ & + & + & 1 & . & + & + & . & + \\
\hline Tritonia x crocosmiflora & . & . & . & + & $\cdot$ & + & + & 3 & . & + & $\cdot$ & . & . & . & . & $\cdot$ & + & + & 1 & 1 & . & . & . & · \\
\hline Geum urbanum & . & . & + & + & . & . & + & + & + & 1 & . & . & . & + & . & 1 & 1 & . & . & . & . & . & . & . \\
\hline Ruscus aculeatus & + & . & . & $\cdot$ & $\cdot$ & $\cdot$ & + & · & + & + & $\cdot$ & . & · & · & + & $\cdot$ & + & . & 1 & + & . & . & + & . \\
\hline Chrysosplenium oppositifolium & . & . & 1 & . & + & $\cdot$ & . & . & . & . & $\cdot$ & + & . & + & . & + & + & . & + & + & · & · & · & . \\
\hline Glechoma hederacea & . & . & . & . & 1 & 1 & 1 & . & 1 & + & . & . & . & . & . & + & . & . & . & . & + & . & . & . \\
\hline Heracleum sphondylium & . & . & . & . & . & 1 & . & . & . & + & . & . & . & + & 1 & 1 & 1 & . & + & . & . & . & . & . \\
\hline Umbilicus rupestris & . & . & . & . & . & . & + & . & . & . & . & . & . & + & . & 1 & . & + & + & . & 1 & + & . & . \\
\hline Prunella vulgaris & . & . & . & 1 & + & . & . & . & . & + & . & + & . & . & . & + & . & . & . & + & . & . & . & + \\
\hline Digitalis purpurea & + & 1 & . & + & . & . & . & 1 & . & . & $r$ & $r$ & . & . & . & . & . & . & . & . & . & . & . & . \\
\hline Carex laevigata & + & . & + & . & . & . & . & . & . & . & + & . & . & + & . & . & . & + & . & . & . & . & 1 & . \\
\hline Lapsana communis & . & . & . & 1 & $\cdot$ & . & . & . & . & . & . & . & . & + & 1 & $\cdot$ & . & . & . & . & . & . & + & . \\
\hline Valeriana dioica & . & . & . & . & . & . & . & . & . & . & 1 & + & . & . & . & + & . & 1 & . & . & · & . & · & · \\
\hline Angelica major & + & . & . & . & . & . & . & + & . & . & + & . & . & . & . & . & . & . & . & . & 1 & . & . & . \\
\hline Mentha aquatica & . & . & . & . & + & . & . & . & + & + & . & . & . & . & . & + & . & . & . & . & . & . & . & . \\
\hline Polygonum persicaria & . & . & . & $r$ & + & + & . & . & . & + & . & . & . & . & . & . & . & . & . & . & . & . & . & . \\
\hline Cruciataglabra & . & . & . & . & . & . & . & . & . & . & $r$ & . & + & . & . & $\cdot$ & . & . & . & . & + & + & . & \\
\hline
\end{tabular}


Taxones presentes en menos de cuatro inventarios:

$\mathrm{E}_{1}+\mathrm{E}_{2}$ : Acacia dealbata: 1 en 22; Acacia melanoxylon: $\mathrm{r}$ en 8; Cytisus scoparius: $\mathrm{r}$ en 12 y $\mathrm{r}$ en 13; Prunus avium: + en 12: Prunus laurocerasus: r en 3; Prunus spinosa: + en 20; Rosa gr. canina: + en 7 y + en 15; Rosa micrantha: + en 17; Taxus baccata: r en 16; Ulmus glabra: + en 12. Taxones característicos de asociación y unidades superiores: Carex sylvatica: + en 17; Ranunculus tuberosus: + en 8, + en 15 y + rn 17; Solanum dulcamara: + en 6, + en 10 y + en 17; Symphytum tuberosum: 3 en 7, 3 en 17 y + en 19. Taxones característicos de Quercetea robori-petraeae y Carpino-Fagetea: Conopodium majus: + en 17; Dryopteris aemula: 1 en 12; Polygonatum verticillatum: + en 17. Otros taxones: Agrostis capillaris: + en 1, + en 12 y 1 en 13; Allium victorialis: 2 en $13,+$ en 19 y + en 23; Anthoxanthum amarum: $\mathrm{r}$ en 15 y + en 17; Apium nodiflorum: + en 9 y + en 18; Arrhenatherum bulbosum: 1 en 13,1 en 22 y + en 23; Asphodelus lusitanicus: + en 19 y r en 22; Calystegia sepium: + en 5; Cardamine flexuosa: + en 5 y 1 en 8; Cardamine hirsuta: + en 4, + en 16 y + en 17; Centaurea rivularis: + en 1, + en 2 y 1 en 23; Cirsium palustre: + en 20 y + en 22; Crocus serotinus: + en 19; Daboecia cantabrica: + en 1; Epilobium sp.: + en 4 y + en 7; Eupatorium cannabinum: + en 17; Galium aparine:+ en 7, + en 10 y + en 17; Galium palustre: + en 10; Hydrangea macrophylla: + en 7; Hypericum tetrapterum: + en 5; Juncus effusus: + en 1, + en 7 y r en $9 ;$ Limniris pseudacorus: + en 18; Lythrum salicaria: + en 6 y + en 10; Mentha pulegium: + en 17; Molinia caerulea: 1 en 13; Narcissus bulbocodium: + en 15; Oxalis pes-caprae: + en 10; Pentaglottis sempervirens: + en 7; Peucedanum gallicum: + en 4; Peucedanum lancifolium: + en 21; Picris hieracioides: + en 1 y + en 8; Plantago lanceolata: + en 13; Polygonatum odoratum: + en 19; Polypodium cambricum: 1 en 8; Potentilla erecta: + en 22; Sibthorpia europea: + en 8 y + en 12; Sparganium neglectum: +en 10; Taraxacum gr. officinale: + en 2 y + en 8; Tradescantia fluminensis: + en 5 y 2 en 10; Ulex europaeus: + en 1; Urtica dioica: 1 en 3, 1 en 7 y + en 9.

Procedencia de los inventarios:

1: As: Coaña, Nadou, Río Meiro (678/4817); 2: As: Coaña, Río Meiro, aguas arriba de Meiro (681/4820); 3: As: Cudillero, Río de Piñera, aguas arriba del pueblo (730/4825); 4: As: Cudillero, Río Esqueiro, aguas abajo del cruce a Foyedo (719/4823); 5: As: Cudillero, Río Esqueiro, aguas abajo del puente entre Salamir y San Pedro (724/4827); 6: As: Cudillero, Río Uncín, aguas arriba de Los Pradones (727/4827); 7: As: La Caridad, Arancedo, Río del Mazo, augas arriba de Boimouro (674/4819); 8: As: Navia, Río Barayo, aguas abajo de El Bao (692/4825); 9: As: Valdés, Río de Carcedo, entre Muñás de Baxu y Brieves (708/4817); 10: As: Valdés, Río Esba, entre El Ranón y Trevias (707/4821); 11: C: Aranga, Rego de Portorrosa, entre Pousadoiro y A Bouza (581/4784); 12: C: Aranga, Río de Cambás, por encima de la Central Eléctrica de Congostro (581/4787); 13: C: As Pontes, entre A Ribeira y As Pontes de García Rodríguez (594/4812); 14: C: As Somozas, Rego da Fonte de Rámez, entre A Ponte do Batán y su unión con el Rego de Escalo (585/4819); 15: C: As Somozas, Río Mera, aguas arriba de la Capela do Silvao (588/4825); 16: C: As Somozas, Río Pequeno, entre Coucixoso e O Condado (585/4818); 17: C: Cerdido, A Barqueira, entre O Covelo e A Cuqueira, Río do Loureiro (587/4828); 18: C: Cerdido, Díaz, Rego da Pontellas (582/4830); 19: C: Cerdido, entre a Campeira e A Gangoa, Río do Porto do Cabo (580/4828); 20: C: Cerdido, entre Penso e O Carballeiro, margen derecha del Rego do Porto do Cabo (579/4829); 21: C: Fene, entre San Marcos e A Ribeira, aguas arriba de Ponte da Ribeira, margen derecha del Río Belelle (571/4812); 22: C: Irixoa, Ambroá, margen izquierda del Río Lambre, aguas abajo de Ponte de Moreira (572/4796); 23: C: Mañón, Río Sor, aguas abajo de Ribeiras do Bispo (603/4826); 24: C: Mañón, Río Sor, margen derecha aguas arriba de Ponte Segade (602/4829). 
Tabla IVb. Alisedas cantábricas occidentales (Valeriano pyrenaicae-Alnetum glutinosae) variante típica, subvariante típica (cont.)

\begin{tabular}{|c|c|c|c|c|c|c|c|c|c|c|c|c|c|c|c|c|c|c|c|c|c|c|c|c|}
\hline $\mathrm{N}^{0}$ de orden & 1 & 2 & 3 & 4 & 5 & 6 & 7 & 8 & 9 & 10 & 11 & 12 & 13 & 14 & 15 & 16 & 17 & 18 & 19 & 20 & 21 & 22 & 23 & 24 \\
\hline Altitud (m) & 45 & 310 & 325 & 20 & 10 & 35 & 15 & 45 & 35 & 15 & 35 & 85 & 175 & 210 & 45 & 35 & 25 & 5 & 575 & 385 & 455 & 145 & 190 & 125 \\
\hline Pendiente $\left({ }^{\circ}\right)$ & $<2$ & 2 & 2 & $<2$ & 2 & $<2$ & 4 & $<2$ & 4 & $<2$ & 2 & $<2$ & $<2$ & 4 & $<2$ & $<2$ & $<2$ & 4 & $<2$ & 6 & $<2$ & 4 & 10 & $<2$ \\
\hline Orientación & NNW & NE & W & SW & NNE & $\mathrm{N}$ & NW & NNE & NNW & NNE & NNW & $\mathrm{NE}$ & $\mathrm{N}$ & WNW & ENE & NNW & WSW & I $S$ & SSE & WSW & W & WSW & $\mathrm{E}$ & SE \\
\hline Altura de copas (m) & 18 & 18 & 20 & 20 & 22 & 13 & 14 & 18 & 26 & 14 & 24 & 20 & 18 & 16 & 20 & 16 & 18 & 16 & 20 & 18 & 12 & 18 & 22 & 18 \\
\hline Cob. $E_{1}(\%)$ & 90 & 90 & 100 & 100 & 85 & 100 & 100 & 100 & 100 & 100 & 100 & 90 & 100 & 90 & 95 & 90 & 100 & 90 & 100 & 95 & 100 & 95 & 90 & 100 \\
\hline Cob. $E_{2}(\%)$ & 30 & 15 & 15 & 25 & 30 & 25 & 20 & 15 & 30 & 14 & 40 & 40 & 35 & 20 & 35 & 35 & 20 & 15 & 60 & 30 & 30 & 15 & 20 & 15 \\
\hline Cob. $E_{3}(\%)$ & 95 & 100 & 90 & 95 & 85 & 80 & 85 & 95 & 90 & 90 & 90 & 90 & 100 & 90 & 90 & 100 & 100 & 75 & 90 & 50 & 100 & 75 & 60 & 90 \\
\hline Área $\left(m^{2}\right)$ & 300 & 300 & 300 & 300 & 400 & 240 & 300 & 300 & 500 & 500 & 300 & 400 & 300 & 300 & 500 & 200 & 400 & 300 & 300 & 400 & 500 & 300 & 200 & 300 \\
\hline$N^{0}$ de taxones & 45 & 35 & 41 & 56 & 63 & 52 & 49 & 47 & 86 & 68 & 71 & 80 & 45 & 51 & 59 & 55 & 63 & 36 & 60 & 62 & 39 & 57 & 36 & 73 \\
\hline \multicolumn{25}{|c|}{$E_{1}(>4,0 m)+E_{2}(>1,5-4,0 n$} \\
\hline Alnus glutinosa & 3 & 4 & 4 & 5 & 4 & 4 & 4 & 5 & 4 & 4 & 5 & 3 & 4 & $r$ & 5 & 4 & 5 & 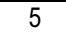 & 5 & 4 & 4 & 4 & 4 & 4 \\
\hline Laurus & 1 & 1 & 1 & 1 & 2 & 1 & 2 & 1 & 1 & 3 & 1 & 1 & 1 & 1 & 2 & 1 & 1 & 2 & 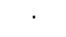 & 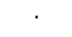 & . & 1 & 2 & + \\
\hline Frax & . & . & . & 2 & 2 & 1 & + & + & 3 & 2 & 2 & 3 & 3 & 1 & 2 & 2 & 1 & 2 & 1 & 2 & 2 & 2 & . & 1 \\
\hline Salix atrocinerea & 2 & 1 & . & 1 & 2 & 1 & 1 & 1 & 1 & 2 & 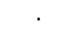 & 1 & 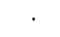 & 1 & 1 & 1 & 1 & & 1 & 2 & 2 & 1 & 2 & 1 \\
\hline Sambucus nigra & . & . & . & + & 1 & 1 & 1 & 1 & + & + & + & + & + & 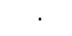 & + & + & 1 & + & 1 & + & 1 & 1 & 1 & 1 \\
\hline Corylus avellana & 1 & . & . & 1 & 1 & 1 & 2 & . & 1 & 2 & + & 1 & 3 & 1 & 2 & 1 & 2 & . & . & 2 & . & 2 & 1 & \\
\hline Quer & 1 & 1 & 3 & 1 & + & 2 & & . & 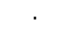 & 1 & . & + & 1 & 1 & + & . & + & . & . & 1 & $r$ & . & . & + \\
\hline Acerp & 1 & $\mathrm{r}$ & . & 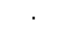 & . & . & 2 & . & 2 & 3 & 1 & 2 & 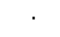 & 1 & . & + & . & 1 & 1 & 1 & . & + & . & 1 \\
\hline Castanea sativa & 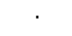 & . & 1 & 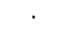 & . & + & & 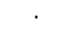 & + & + & . & $r$ & . & & 1 & . & $r$ & 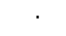 & . & 1 & . & 1 & 1 & . \\
\hline Crataegus monogyna & 1 & . & . & . & . & . & 1 & 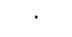 & + & + & . & + & + & & 1 & $\theta$ & 1 & 1 & + & . & . & . & . & . \\
\hline Ligustrum o & . & . & . & 1 & . & 1 & 1 & $r$ & + & 1 & . & 1 & . & . & 1 & 1 & 1 & 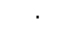 & . & . & . & . & . & 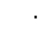 \\
\hline Frangl & 1 & + & + & . & . & . & . & . & + & . & . & + & + & + & + & . & + & . & . & 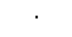 & . & . & . & 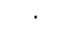 \\
\hline Pyrus cordata & 1 & 1 & + & . & . & + & 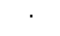 & . & . & + & + & . & + & . & . & . & . & . & . & . & . & & . & . \\
\hline Ilex aquifolium & . & . & . & . & . & . & 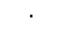 & . & 1 & . & + & + & + & . & + & 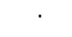 & 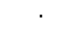 & 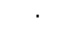 & . & r & . & . & . & 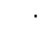 \\
\hline Eric & . & + & + & 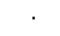 & . & + & & . & . & . & . & . & . & + & . & 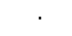 & . & . & . & + & . & . & . & \\
\hline Rosa gr. canina & & & . & + & . & . & & . & . & & + & . & . & & . & + & . & & 1 & 1 & . & . & . & \\
\hline
\end{tabular}

$\mathrm{E}_{3}(<1,5 \mathrm{~m})$ :

Taxones diferenciales frente a Senecioni-Alnetum

Festuca gigantea

Salix fragilis

Lastrea limbosperma

Narcissus pseudonarcissus s.l.

Cardamine raphanifolia

\begin{tabular}{|c|c|c|c|c|c|c|c|c|c|c|c|c|c|c|c|c|c|c|c|c|c|c|c|c|}
\hline \multicolumn{25}{|c|}{ Taxones diferenciales frente a Hyperico-Alnetum } \\
\hline Hedera hibernica & 2 & 2 & 2 & 1 & 2 & 3 & 3 & 2 & 3 & 2 & 2 & 2 & 1 & 1 & 3 & 2 & 2 & 2 & 2 & 1 & 3 & 2 & 2 & 2 \\
\hline Carex reuteriana & + & . & 1 & . & + & 2 & 1 & . & + & + & + & + & $\cdot$ & 2 & 1 & . & + & 1 & 1 & 1 & . & + & $\cdot$ & . \\
\hline Viola palustris & 2 & 1 & 1 & . & . & 1 & + & + & + & . & + & + & + & 2 & 1 & + & . & 1 & . & + & . & $\cdot$ & $\cdot$ & . \\
\hline Omphalodes nitida & . & 1 & + & + & . & + & + & + & . & . & 1 & 1 & . & . & + & + & + & $r$ & . & . & . & . & + & . \\
\hline Deschampsia subtriflora & 2 & 1 & + & . & . & + & + & $\cdot$ & $r$ & . & + & . & + & 2 & 1 & . & + & . & . & . & . & . & . & . \\
\hline Saxifraga spathularis & 2 & 2 & . & . & . & . & . & . & 1 & . & 1 & + & $\cdot$ & 1 & 1 & . & 1 & $\cdot$ & . & . & . & + & 1 & . \\
\hline Betula pubescens & 1 & 2 & 2 & . & 1 & . & . & . & . & . & . & + & $\cdot$ & 1 & 1 & 1 & . & . & . & . & . & . & . & 1 \\
\hline Narcissus triandrus & + & + & · & . & . & + & + & + & + & . & + & $\cdot$ & $\cdot$ & $\cdot$ & + & . & . & $\cdot$ & $\cdot$ & . & . & . & $\cdot$ & . \\
\hline Dryopteris aemula & + & . & 1 & . & . & . & . & . & . & . & . & . & . & 1 & + & . & . & . & . & . & . & . & . & . \\
\hline Narcissus asturiensis & . & . & . & . & . & + & + & . & . & . & . & . & . & + & . & . & . & . & . & . & . & . & . & . \\
\hline Scrophularia alpestris & . & . & . & . & . & . & . & . & . & . & . & . & $\cdot$ & . & $\cdot$ & . & . & $\cdot$ & . & 1 & . & . & $\cdot$ & . \\
\hline Helleborus foetidus & . & . & . & . & . & . & . & . & . & . & . & . & $\cdot$ & . & $\cdot$ & . & . & $\cdot$ & . & + & . & . & $\cdot$ & . \\
\hline Adenostyles alpina & . & . & . & . & . & . & . & . & + & . & . & . & . & . & . & . & . & . & . & . & . & . & . & . \\
\hline Solidago virgaurea & $\cdot$ & . & . & . & . & + & . & . & . & . & . & . & $\cdot$ & . & . & . & . & . & . & . & . & . & . & . \\
\hline Cytisus commutatus & . & . & + & . & . & . & . & . & . & . & . & . & . & . & . & . & . & . & $\cdot$ & . & . & . & $\cdot$ & \\
\hline \multicolumn{25}{|c|}{ Taxones característicos de asociación y unidades superiores } \\
\hline Athyrium filix-femina & 2 & 2 & 2 & 1 & 1 & 3 & 2 & 2 & 2 & 1 & 3 & 2 & 2 & 2 & 1 & 1 & 1 & 3 & 1 & 2 & 2 & 1 & 2 & 2 \\
\hline Viola riviniana & 1 & 1 & + & 1 & 2 & 1 & 1 & 1 & 1 & 1 & 1 & + & $\cdot$ & + & 1 & 1 & 1 & 2 & 1 & + & + & + & 1 & + \\
\hline Osmunda regalis & 4 & . & + & 2 & 2 & 2 & 3 & 1 & 2 & 2 & 2 & 1 & 1 & 2 & 1 & 2 & 3 & 3 & . & . & . & + & 1 & 1 \\
\hline Dryopteris affinis & + & 2 & 2 & 1 & 1 & + & 1 & . & 2 & 1 & 1 & 1 & 1 & 2 & 2 & . & 1 & . & . & 1 & 1 & 1 & 1 & + \\
\hline Ranunculus ficaria & $\cdot$ & . & . & 3 & 2 & + & 1 & 2 & 1 & 3 & 1 & 2 & 1 & + & 1 & 1 & 1 & 2 & 1 & + & . & 1 & $\cdot$ & 3 \\
\hline Blechnum spicant & 2 & 1 & 1 & + & + & 1 & + & + & 1 & + & 1 & + & $\cdot$ & 2 & 1 & . & 1 & . & $\cdot$ & 1 & . & . & 1 & + \\
\hline Euphorbia dulcis & + & 2 & 1 & . & 1 & 1 & 1 & . & 2 & + & 3 & 3 & + & 1 & 1 & + & 1 & . & . & 1 & . & . & 2 & + \\
\hline
\end{tabular}




\begin{tabular}{|c|c|c|c|c|c|c|c|c|c|c|c|c|c|c|c|c|c|c|c|c|c|c|c|c|}
\hline $\mathrm{N}^{0}$ de orden & 1 & 2 & 3 & 4 & 5 & 6 & 7 & 8 & 9 & 10 & 11 & 12 & 13 & 14 & 15 & 16 & 17 & 18 & 19 & 20 & 21 & 22 & 23 & 24 \\
\hline Lonicera hispanica & + & 1 & 1 & + & $\cdot$ & 1 & . & 1 & + & + & $\cdot$ & 1 & + & 1 & 1 & $\cdot$ & 2 & - & 1 & $\cdot$ & 1 & $\cdot$ & 1 & 1 \\
\hline Primula acaulis & . & . & . & 1 & 2 & + & 1 & 1 & 2 & 1 & 1 & 1 & . & + & 1 & + & + & 2 & · & $\cdot$ & + & . & + & . \\
\hline Polystichum setiferum & . & . & . & 1 & 2 & + & . & . & + & 1 & 1 & 2 & + & . & 1 & + & . & 1 & 1 & . & . & 1 & 1 & 2 \\
\hline Brachypodium sylvaticum & + & . & . & 1 & . & . & . & 2 & 1 & 1 & + & 1 & 1 & $\cdot$ & $\cdot$ & + & . & 1 & 1 & 1 & . & 1 & + & 2 \\
\hline Hypericum androsaemum & . & . & . & + & + & + & + & + & + & + & 1 & + & . & . & + & + & + & . & $\cdot$ & . & $\cdot$ & 1 & 1 & 1 \\
\hline Oxalis acetosella & + & 1 & + & + & . & · & + & . & 1 & 1 & 2 & 2 & . & $\cdot$ & 1 & . & 1 & $\cdot$ & $\cdot$ & + & . & + & 1 & . \\
\hline Carex remota & . & . & . & + & + & . & . & . & + & + & + & + & + & $\cdot$ & 1 & . & . & $\cdot$ & 1 & 1 & $\cdot$ & 1 & + & 1 \\
\hline Carex pendula & . & . & . & + & 1 & . & . & . & + & 1 & 1 & 1 & . & . & . & $r$ & . & + & $\cdot$ & 1 & $\cdot$ & 1 & + & 2 \\
\hline Senecio nemorensis & + & 1 & . & 1 & 1 & . & + & 1 & . & . & . & + & 1 & 1 & + & . & 1 & + & . & . & $\cdot$ & . & . & . \\
\hline Luzula sylvatica & + & . & . & . & . & . & 1 & . & + & + & + & . & . & + & 1 & . & 1 & . & . & 1 & $\cdot$ & . & . & . \\
\hline Symphytum tuberosum & . & . & $\cdot$ & 1 & · & . & · & 1 & + & 2 & + & $\cdot$ & + & $\cdot$ & $\cdot$ & 1 & + & $\cdot$ & $\cdot$ & $\cdot$ & $\cdot$ & $\cdot$ & . & 1 \\
\hline Valeriana pyrenaica & . & · & $\cdot$ & . & · & . & · & . & 1 & + & 1 & + & $\cdot$ & $\cdot$ & $\cdot$ & $\cdot$ & · & $\cdot$ & $\cdot$ & 1 & $\cdot$ & 1 & 1 & 1 \\
\hline Arum italicum & . & . & . & 1 & · & . & · & . & . & + & . & + & $\cdot$ & $\cdot$ & $\cdot$ & + & & + & $\cdot$ & . & $\cdot$ & + & 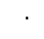 & 1 \\
\hline
\end{tabular}

Taxones característicos de Quercetea robori-petraeae y Carpino-Fagetea

\begin{tabular}{|c|c|c|c|c|c|c|c|c|c|c|c|c|c|c|c|c|c|c|c|c|c|c|c|c|}
\hline Holcus mollis & $\cdot$ & 2 & 1 & 1 & 1 & + & 1 & 1 & + & . & 1 & + & 1 & 1 & 1 & 1 & 2 & . & + & $\cdot$ & 1 & + & 1 & + \\
\hline Ajuga reptans & 1 & . & $\cdot$ & · & + & 1 & + & + & 1 & + & + & 1 & + & + & + & $\cdot$ & 1 & + & . & 1 & . & + & + & · \\
\hline Teucrium scorodonia & + & 1 & 1 & + & + & . & + & + & + & . & 1 & 1 & . & 1 & + & $r$ & + & $r$ & . & $\cdot$ & . & . & + & . \\
\hline Dryopteris dilatata & + & . & . & + & + & + & . & 1 & 1 & $r$ & + & 1 & 1 & 1 & 1 & . & 1 & . & . & $\cdot$ & . & + & $\cdot$ & 1 \\
\hline Anemone nemorosa & 2 & . & · & 1 & 1 & 2 & 2 & 2 & 3 & 2 & 2 & 2 & · & 1 & 2 & 1 & $\cdot$ & 1 & . & $\cdot$ & · & . & $\cdot$ & + \\
\hline Euphorbia amygdaloides & $\cdot$ & · & . & + & + & . & + & 1 & + & 1 & 1 & + & + & . & + & + & $\cdot$ & + & + & $\cdot$ & + & . & $\cdot$ & + \\
\hline Hyacinthoides non-scripta & $\cdot$ & . & . & + & + & 1 & 1 & 2 & 1 & + & 1 & + & . & 1 & 3 & + & + & . & . & $\cdot$ & . & . & $\cdot$ & + \\
\hline Polypodium vulgare & · & + & 1 & . & · & + & . & . & + & . & + & + & . & 1 & + & . & 1 & + & + & + & . & . & $\cdot$ & 1 \\
\hline Lysimachia nemorum & $\cdot$ & $\cdot$ & $\cdot$ & 1 & 1 & . & . & 2 & + & · & + & 1 & + & · & · & + & 1 & . & . & $\cdot$ & + & . & $\cdot$ & 1 \\
\hline Mercurialis perennis & $\cdot$ & $\cdot$ & . & 1 & 2 & . & 1 & . & 1 & 2 & 1 & 1 & · & · & · & 1 & $\cdot$ & · & . & $\cdot$ & 1 & + & + & · \\
\hline Veronica montana & . & . & . & 1 & + & . & . & . & 1 & 1 & + & 2 & 1 & . & . & 1 & + & . & . & + & . & + & . & . \\
\hline Pulmonaria longifolia & $\cdot$ & . & . & 1 & + & + & + & . & 1 & 1 & 1 & $\cdot$ & . & . & . & . & + & + & . & $\cdot$ & . & . & . & $\cdot$ \\
\hline Potentilla sterilis & $\cdot$ & · & . & 1 & · & . & + & . & + & 1 & + & + & $\cdot$ & + & . & + & . & + & . & $\cdot$ & · & . & . & . \\
\hline Stachys sylvatica & · & · & . & . & + & . & · & . & + & + & + & 1 & + & . & · & + & + & . & + & $\cdot$ & · & . & · & $\cdot$ \\
\hline Aquilegia vulgaris & $\cdot$ & . & . & . & · & + & + & . & + & · & . & + & · & . & . & + & + & · & . & $\cdot$ & · & . & + & + \\
\hline Crepis lampsanoides & + & . & . & . & . & . & . & . & + & . & . & + & . & . & 1 & . & + & . & + & + & . & . & . & + \\
\hline Tamus communis & $\cdot$ & · & . & . & - & + & + & + & + & + & . & $\cdot$ & $\cdot$ & . & . & + & $\cdot$ & . & + & $\cdot$ & $\cdot$ & . & $\cdot$ & . \\
\hline Stellaria holostea & $\cdot$ & · & + & + & · & . & · & · & + & $\cdot$ & 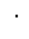 & + & · & 1 & · & . & . & · & 1 & $\cdot$ & · & . & · & $\cdot$ \\
\hline Ranunculus tuberosus & + & . & . & . & - & . & · & · & 1 & + & + & $\cdot$ & $\cdot$ & . & . & . & 1 & · & . & $\cdot$ & $\cdot$ & . & . & + \\
\hline Carex sylvatica & · & · & . & . & · & · & $\cdot$ & · & 1 & + & + & + & $\cdot$ & · & · & + & . & · & . & + & · & . & · & . \\
\hline Conopodium majus & 1 & . & + & . & - & . & . & 1 & + & + & . & $\cdot$ & . & . & . & . & . & · & . & $\cdot$ & $\cdot$ & . & . & . \\
\hline Sanicula europaea & · & · & · & . & + & . & · & . & 1 & · & 1 & 2 & $\cdot$ & · & · & . & . & · & . & $\cdot$ & · & . & . & . \\
\hline Stachys officinalis & $\cdot$ & 1 & . & . & + & + & . & . & + & . & . & $\cdot$ & . & . & . & . & & . & . & $\cdot$ & · & . & . & . \\
\hline
\end{tabular}

\begin{tabular}{|c|c|c|c|c|c|c|c|c|c|c|c|c|c|c|c|c|c|c|c|c|c|c|c|c|}
\hline \multicolumn{25}{|l|}{ Otros taxones } \\
\hline Rubus sp. & 1 & 1 & 1 & . & + & . & + & 1 & + & + & + & 1 & 1 & 1 & + & 1 & 1 & + & 1 & 1 & 2 & 1 & + & $r$ \\
\hline Oenanthe crocata & + & + & 1 & 1 & 1 & + & 2 & 1 & + & + & 2 & 1 & 1 & $\cdot$ & 1 & 2 & 1 & 2 & $\cdot$ & + & + & + & . & 2 \\
\hline Brachypodium rupestre & $\cdot$ & 2 & 2 & 3 & 1 & 1 & 1 & . & + & 1 & + & 1 & 3 & $\cdot$ & 1 & 3 & 2 & . & · & 1 & 4 & $\cdot$ & + & . \\
\hline Geranium robertianum & . & . & . & 1 & 1 & + & + & . & . & + & + & + & + & $\cdot$ & . & $r$ & + & . & 1 & 1 & 1 & 1 & 1 & 2 \\
\hline Pteridum aquilinum & + & + & + & . & + & + & $r$ & . & $\cdot$ & + & $r$ & + & $\cdot$ & 1 & + & . & 1 & . & + & . & + & $r$ & . & + \\
\hline Geum urbanum & $\cdot$ & . & . & 1 & 1 & . & . & . & + & + & + & + & $\cdot$ & $\cdot$ & · & + & + & $r$ & 1 & + & 1 & 1 & + & 2 \\
\hline Angelica sylvestris & . & . & . & 1 & + & . & . & + & 1 & 1 & 1 & + & + & 1 & + & $r$ & $\cdot$ & . & + & . & . & + & . & + \\
\hline Tritonia x crocosmiflora & $\cdot$ & . & . & 2 & 1 & + & 1 & 1 & 1 & 3 & 1 & 1 & $\cdot$ & $\cdot$ & 1 & 1 & $\cdot$ & . & $\cdot$ & $\cdot$ & $\cdot$ & $\cdot$ & . & 1 \\
\hline Silene dioica & . & . & . & . & + & + & . & + & + & . & + & $\cdot$ & . & 1 & . & . & + & . & 1 & 1 & 1 & 2 & . & 1 \\
\hline Anthoxanthum amarum & 1 & . & 1 & 1 & + & . & . & 1 & + & . & + & 1 & . & 1 & + & . & 1 & . & . & . & $\cdot$ & . & . & . \\
\hline Dactylis glomerata & + & + & + & + & + & . & . & . & + & · & . & + & $\cdot$ & $\cdot$ & . & . & $\cdot$ & . & · & $r$ & 1 & + & . & + \\
\hline Rumex acetosa & . & . & . & + & . & . & . & + & . & . & $r$ & + & . & + & . & + & + & . & + & + & + & . & . & . \\
\hline Ruscus aculeatus & + & . & . & . & . & + & . & . & + & + & + & $\cdot$ & $\cdot$ & + & . & . & $\cdot$ & . & $\cdot$ & + & . & + & . & + \\
\hline Lamium maculatum & $\cdot$ & . & . & . & 1 & . & . & + & $\cdot$ & + & . & + & $\cdot$ & $\cdot$ & . & + & + & . & + & . & · & 1 & . & 1 \\
\hline Cardamine pratensis & $\cdot$ & · & . & . & · & $\cdot$ & + & . & + & + & + & + & $\cdot$ & + & $\cdot$ & + & + & + & $\cdot$ & $\cdot$ & $\cdot$ & $\cdot$ & . & · \\
\hline Heracleum sphondylium & . & . & . & . & . & + & + & . & 1 & 1 & 1 & + & . & $\cdot$ & . & . & . & . & + & . & 2 & . & . & . \\
\hline Scrophularia auriculata & . & . & . & . & + & . & . & . & + & . & + & $\cdot$ & . & $\cdot$ & . & . & + & . & + & + & + & + & . & 1 \\
\hline Ranunculus repens & $\cdot$ & . & . & + & . & . & . & + & $\cdot$ & . & . & $\cdot$ & + & $\cdot$ & . & . & + & . & $\cdot$ & . & + & 1 & . & 1 \\
\hline Glechoma hederacea & $\cdot$ & . & . & 1 & - & . & · & . & + & + & + & $\cdot$ & $\cdot$ & $\cdot$ & . & $\cdot$ & $\cdot$ & . & · & $\cdot$ & 2 & $\cdot$ & . & 1 \\
\hline Urtica dioica & . & . & . & . & . & . & . & . & . & . & . & $\cdot$ & . & $\cdot$ & . & $r$ & . & $r$ & 1 & + & 2 & . & . & + \\
\hline Limniris pseudacorus & . & . & . & 1 & + & . & . & . & . & . & . & + & + & $\cdot$ & . & + & . & 1 & . & . & . & . & . & . \\
\hline Lapsana communis & + & . & . & . & . & . & + & . & $\cdot$ & . & + & $\cdot$ & $\cdot$ & $\cdot$ & . & . & $\cdot$ & . & + & . & + & $\cdot$ & . & + \\
\hline Galium aparine & $\cdot$ & · & . & + & + & . & . & . & $\cdot$ & + & . & $\cdot$ & $\cdot$ & $\cdot$ & . & . & $\cdot$ & . & + & . & . & + & . & + \\
\hline Taraxacum gr. officinale & $\cdot$ & . & . & + & + & . & . & . & $r$ & · & $r$ & $\cdot$ & $\cdot$ & $\cdot$ & + & . & $\cdot$ & . & + & . & . & $\cdot$ & . & · \\
\hline Arrhenatherum bulbosum & . & 1 & . & . & . & . & . & . & . & + & . & $\cdot$ & . & 1 & + & . & 1 & . & . & . & . & . & . & . \\
\hline Caltha palustris & 1 & . & . & . & . & . & . & . & . & . & . & + & $\cdot$ & + & + & . & $\cdot$ & + & . & . & . & $\cdot$ & . & · \\
\hline Umbilicus rupestris & . & . & + & . & . & . & + & . & . & . & . & $\cdot$ & $\cdot$ & $\cdot$ & $\cdot$ & $\cdot$ & + & . & . & $\cdot$ & $\cdot$ & + & . & 1 \\
\hline Carex laevigata & + & + & + & . & . & . & . & . & + & . & . & $\cdot$ & $\cdot$ & $\cdot$ & + & . & . & . & $\cdot$ & $\cdot$ & $\cdot$ & $\cdot$ & . & . \\
\hline Digitalis purpurea & . & . & + & . & + & . & . & + & . & . & $r$ & $\cdot$ & $\cdot$ & + & . & . & $\cdot$ & . & . & . & $\cdot$ & $\cdot$ & . & . \\
\hline Equisetum arvense & $\cdot$ & . & . & . & . & . & . & . & · & . & . & $\cdot$ & $\cdot$ & $\cdot$ & $\cdot$ & . & $\cdot$ & . & 1 & 1 & $\cdot$ & + & . & 1 \\
\hline Eupatorium cannabinum & . & . & . & . & . & . & . & . & . & . & . & $\cdot$ & $\cdot$ & $\cdot$ & . & 1 & . & . & + & + & . & + & . & . \\
\hline Centaurea rivularis & $\cdot$ & . & + & . & + & . & . & . & . & . & . & + & $\cdot$ & $\cdot$ & . & . & . & . & . & + & . & . & . & . \\
\hline
\end{tabular}


Taxones presentes en menos de cuatro inventarios:

$\mathrm{E}_{1}+\mathrm{E}_{2}$ : Buxus sempervirens: 1 en 4 y + en 7; Cytisus scoparius: + en 3 y + en 15; Cytisus striatus: + en 14; Juglans regia: + en 19; Prunus avium: + en 23; Prunus laurocerasus: r en 9; Prunus spinosa: 1 en 5, + en 13 y 1 en 24; Quercus pirenaica: 1 en 5; Sorbus torminalis: 1 en 20; Ulmus glabra: + en 9, 1 en 13 y 1 en 20. Taxones característicos de asociación y unidades superiores: Allium scorzonerifolium: + en 16; Circaea lutetiana: + en 9, + en 20 y + en 22; Humulus lupulus: + en 5; Myosotis Martini: + en 9, 1 en 12 y 1 en $24 ;$ Solanum dulcamara: + en 10, 1 en 19 y 2 en 21. Taxones característicos de Quercetea robori-petraeae y Carpino-Fagetea: Dryopteris filix-mas: + en 22; Lathyrus linifolius: + en 6; Melica uniflora: 1 rn 4, 1 en 19 y + en 22; Melittis melissophyllum: + en 6; Moehringia trinervia: + en 12 y + en 20; Phyllitis scolopendrium: + en 17, + en 20 y + en 24; Poa nemoralis: 1 en 19 y + en 20; Vaccinium myrtillus: + en 14. Otros taxones: Alliaria petiolata: + en 19; Angelica major: 1 en 8, + en 15 y 1 en 21; Apium nodiflorum: + en 13, + en 19 y 1 en $24 ;$ Asphodelus lusitanicus: $r$ en 6, + en 8 y + en 9; Asplenium adiantum-nigrum: + en 19; Calystegia sepium: + en 10, + en 19 y + en 21; Cardamine flexuosa: + en 20, + en 23 y 1 en 24; Cardamine hirsuta: + en 20, + en 22 y + en 24; Carex cariophyllea: + en 9; Chrysosplenium oppositifolium: 1 en 20, + en 22 y + en 23; Clematis vitalba: 1 en 9; Conopodium sp.: + en 14 y 1 en 15; Conyza bonariensis: + en 24; Cruciata glabra: + en 3; Cystopteris fragilis: + en 8; Epilobium sp.: + en 19, + en 22 y + en 24; Filipendula ulmaria: + en 12 , 1 en 19 y 3 en 21; Fragaria vesca: + en 19; Galium mollugo: + en 19; Galium palustre: + en 21; Geranium lucidum: + en 22; Glyceria fluitans: $r$ en 19 y + en 20; Hydrangea macrophylla: + en 17; Hypericum tetrapterum: + en 24; Hypericum undulatum: + en 24; Juncus sp.: + en 5 y + en 24; Laserpitium latifolium: + en 20; Leontodon sp.: r en 8; Lotus pedunculatus: + en 19; Lycopus europaeus: + en 12, + en 13 y + en 16; Lythrum salicaria: + en 5, 1 en 16 y + en 19; Mentha aquatica: r en 9, + en 13 y + en 16; Mentha pulegium: + en 5, + en 12 y + en 20; Mentha suaveolens: + en 22 y + en 24; Molinia caerulea: + en 1; Pentaglottis sempervirens: + en 20 y 1 en 24; Phalaris arundinacea: + en 18 y + en 19; Picris hieracioides: + en 4 y + en 24; Polygonatum odoratum: + en 6, + en 9 y + en 10; Polygonum persicaria: + en 20; Polypodium cambricum: + en 22; Prunella vulgaris: + en 9 y + en 13; Rumex obtusifolius: + en 5 y + en 19; Senecio aquaticus: + en 5 y + en 22; Sibthorpia europea: + en 17; Sonchus oleraceus: r en 10 y r en 17; Stellaria graminea: + en 21; Stellaria media: + en 24; Tradescantia fluminensis: + en 18; Valeriana dioica: + en 10, 1 en 12 y + en 17; Veronica chamaedrys: + en 12, + en 13 y + en 21; Vicia sepium: + en 21 e + en 21; Wahlenbergia hederácea: + en 3; Zanthedeschia aethyopica: r en 8.

\section{Procedencia de los inventarios:}

1: C: Mañón, Río Sor, margen izquierda aguas arriba de Ribeiras do Sor (604/4836); 2: C: Monfero, San Fiz, Río Lambre, entre Chao do Viña y O Xestal (577/4795); 3: C: Monfero, Santa Xiá, Río Lambre, entre Cernadas y O Xestal (578/4795); 4: C: Narón, Doso, Río Xubia, entre O Chao ye O Castro (572/4819); 5: C: Narón, Vilaronte, Río Xubia, aguas arriba de Muiño do Loureiro (570/4819); 6: C: Neda, A Gandarela, Río Belelle, aguas arriba de la piscifactoría (570/4817); 7: C: Neda, Río Belelle, entre A Gandarela y O Roxal (570/4817); 8: C: Ortigueira, Couzadoiro, Río Baleo (597/4836); 9: C: Ortigueira, Mera de Arriba, Xudracos, margen derecha del Río Mera (587/4830); 10: C: Ortigueira, Mera, Río Mera, entre Pumariño y Torrente de Arriba (588/4833); 11:C: Ortigueira, Río Mera, entre Fraga y O Cardeal (588/4829); 12: C: San Sadurniño, entre Loureiro e Gradoi, Río Grande de Xubia (582/4820); 13: C: San Sadurniño, entre Tineo y A Veiga, margen izquierda del Rego das Forcadas (576/4826); 14: C: San Sadurniño, Igrexafeita, entre O Lombo y Solposto, Río Castro (579/4815); 15: C: San Sadurniño, Naraío, Río Castro, aguas abajo de Ponte da Ferrería (574/4816); 16: C: San Sadurniño, Río Grande de Xubia (574/4820); 17: C: Vilarmaior, Goimil, Río Lambre, margen derecha augas abaixo de la Central Hidroeléctrica de Goimil (569/4796); 18: C: Xubia, Río Xubia, margen derecha aguas abajo de la Presa do Rei (569/4819); 19: Le: Trabadelo, Río Valcárce, aguas abajo de Pereje (676/4722); 20: Lu: A Fonsagrada, Río da Veiga de Logares, entre Vilardíaz y Gromaz (653/4787); 21: Lu: A Pastoriza, Crecente, Río Miño (636/4789); 22: Lu: A Pontenova, Bogo, O Machuco, Río de Reigadas (649/4797); 23: Lu: A Pontenova, Fraga de Foxas, Rego de Parada (645/4802); 24: Lu: A Pontenova, Roxas, Rego de Riotorto (643/4800). 
Tabla IVc. Alisedas cantábricas occidentales (Valeriano pyrenaicae-Alnetum glutinosae) variante típica, subvariante típica (cont.)

\begin{tabular}{|c|c|c|c|c|c|c|c|c|c|c|c|c|c|c|c|c|c|c|c|c|c|c|c|c|}
\hline $\begin{array}{l}N^{0} \text { de orden } \\
\text { Altitud }(m)\end{array}$ & $\begin{array}{c}1 \\
465\end{array}$ & $\begin{array}{c}2 \\
50\end{array}$ & $\begin{array}{c}3 \\
340\end{array}$ & $\begin{array}{c}4 \\
630\end{array}$ & $\begin{array}{c}\mathbf{5} \\
525\end{array}$ & $\begin{array}{c}6 \\
585\end{array}$ & $\begin{array}{c}7 \\
360\end{array}$ & $\begin{array}{c}8 \\
550\end{array}$ & $\begin{array}{c}9 \\
550\end{array}$ & $\begin{array}{c}10 \\
500\end{array}$ & $\begin{array}{c}11 \\
2\end{array}$ & $\begin{array}{l}12 \\
50\end{array}$ & $\begin{array}{l}13 \\
95\end{array}$ & $\begin{array}{c}14 \\
155\end{array}$ & $\begin{array}{c}15 \\
205\end{array}$ & $\begin{array}{c}16 \\
560\end{array}$ & $\begin{array}{c}17 \\
5\end{array}$ & $\begin{array}{c}18 \\
135\end{array}$ & $\begin{array}{c}19 \\
335\end{array}$ & $\begin{array}{c}20 \\
320\end{array}$ & $\begin{array}{c}21 \\
400\end{array}$ & $\begin{array}{c}22 \\
315\end{array}$ & $\begin{array}{c}23 \\
190\end{array}$ & $\begin{array}{c}24 \\
475\end{array}$ \\
\hline Pendiente $\left({ }^{\circ}\right)$ & 2 & $<2$ & $<2$ & $<2$ & 2 & 2 & 2 & 4 & 2 & 2 & 2 & $<2$ & $<2$ & 4 & 2 & 2 & $<2$ & 6 & 10 & 16 & 10 & 4 & 2 & 4 \\
\hline Orientación & SSE & $\mathrm{E}$ & WNW & SSW & SSE & $\mathrm{N}$ & ENE & SE & SSE & SW & W & $\mathrm{E}$ & NNE & $\mathrm{N}$ & $\mathrm{N}$ & SW & SE & $\mathrm{NE}$ & NNE & NNW & $E$ & $\mathrm{NE}$ & $\mathrm{N}$ & NNE \\
\hline Altura de copas (m) & 28 & 14 & 14 & 22 & 16 & 20 & 22 & 18 & 22 & 18 & 26 & 14 & 18 & 14 & 12 & 20 & 26 & 20 & 14 & 18 & 12 & 14 & 22 & 20 \\
\hline Cob. $E_{1}(\%)$ & 100 & 85 & 90 & 100 & 90 & 95 & 100 & 100 & 100 & 100 & 100 & 95 & 100 & 100 & 90 & 100 & 90 & 100 & 80 & 90 & 90 & 100 & 100 & 100 \\
\hline Cob. $E_{2}(\%)$ & 20 & --- & 10 & 30 & 15 & 20 & 40 & 15 & 20 & 15 & 15 & --- & 20 & 15 & 15 & 25 & 25 & 25 & 15 & 10 & 20 & 15 & 15 & 20 \\
\hline Cob. $E_{3}(\%)$ & 100 & 70 & 90 & 100 & 95 & 95 & 90 & 95 & 100 & 95 & 90 & 80 & 85 & 90 & 90 & 90 & 90 & 80 & 50 & 70 & 70 & 95 & 90 & 90 \\
\hline Área $\left(m^{2}\right)$ & 500 & 500 & 150 & 200 & 400 & 300 & 400 & 120 & 400 & 500 & 300 & 200 & 300 & 300 & 300 & 500 & 450 & 400 & 200 & 200 & 300 & 300 & 300 & 400 \\
\hline$N^{0}$ de taxones & 53 & 53 & 55 & 41 & 61 & 54 & 50 & 45 & 61 & 63 & 43 & 46 & 50 & 47 & 45 & 68 & 79 & 49 & 41 & 22 & 47 & 42 & 54 & 40 \\
\hline \multicolumn{25}{|l|}{$E_{1}(>4,0 \mathrm{~m})+E_{2}(>1,5-4,0 \mathrm{~m}):$} \\
\hline Alnus glutinosa & 4 & 2 & 5 & 5 & 4 & 4 & 4 & 3 & 5 & 5 & 4 & 3 & 5 & 5 & 4 & 3 & 4 & 4 & 4 & 5 & 3 & 4 & 5 & 5 \\
\hline Salix atrocinerea & 1 & 2 & 1 & & 2 & 1 & 1 & 1 & 1 & 1 & + & + & 1 & 1 & 2 & 2 & 1 & 1 & $r$ & . & 2 & + & . & 1 \\
\hline Fraxinus excelsior & + & 1 & 1 & . & 1 & 2 & 3 & 3 & . & 2 & 1 & . & + & . & . & 4 & 2 & 3 & 2 & 1 & 3 & 2 & 1 & + \\
\hline Corylus avellana & & & . & & & + & 2 & 1 & 1 & + & 1 & + & 1 & 1 & + & 1 & 1 & + & 1 & 1 & 2 & 1 & 1 & 1 \\
\hline Sambucus nigra & 1 & 1 & 1 & 2 & 1 & 1 & 2 & 1 & . & + & + & + & 1 & . & . & . & 1 & 1 & · & . & + & 1 & 1 & . \\
\hline Acer pseudoplatanus & 2 & 1 & 1 & . & + & . & 1 & $\cdot$ & $\cdot$ & + & 2 & $\cdot$ & 2 & $\cdot$ & $\cdot$ & 3 & 2 & + & 1 & $\cdot$ & $\cdot$ & 1 & 1 & $\cdot$ \\
\hline Laurus nobilis & & + & $r$ & . & . & . & + & . & . & . & 1 & + & + & + & + & & 2 & . & 2 & 1 & . & + & 1 & . \\
\hline Quercus robur & + & 1 & . & . & 1 & + & + & . & . & . & . & 1 & . & 1 & 1 & & + & $\cdot$ & 3 & . & . & . & 1 & 1 \\
\hline Castanea sativa & + & $\cdot$ & . & . & 1 & . & 1 & $\cdot$ & 1 & + & . & 1 & $\cdot$ & . & $\cdot$ & 1 & & $\cdot$ & 1 & $\cdot$ & . & · & 1 & $\cdot$ \\
\hline Frangula alnus & & . & 1 & + & - & $\cdot$ & $\cdot$ & $\cdot$ & 1 & + & $\cdot$ & 1 & . & + & 1 & & $\cdot$ & $\cdot$ & + & $\cdot$ & 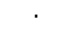 & $\cdot$ & $\cdot$ & 1 \\
\hline Rosa gr. canina & & . & + & + & & + & 1 & . & . & + & . & . & . & . & . & & & . & . & . & + & . & . & 1 \\
\hline Crataegus monogyna & 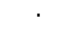 & . & 1 & . & . & . & . & . & 1 & + & . & $\cdot$ & . & . & . & 1 & 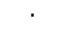 & - & . & . & . & . & . & 1 \\
\hline Ilex aquifolium & . & . & . & . & . & . & . & . & . & . & . & . & . & . & $r$ & . & . & . & 1 & . & + & . & 1 & . \\
\hline Erica arborea & & & $r$ & . & 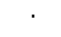 & . & . & . & . & . & . & . & . & + & 1 & & & . & + & . & 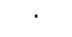 & . & . & $\cdot$ \\
\hline Prunus avium & & & . & + & & & 1 & . & + & . & . & . & . & . & . & $r$ & & $\cdot$ & . & . & & . & . & $\cdot$ \\
\hline \multicolumn{25}{|c|}{$\begin{array}{l}E_{3}(<1,5 \mathrm{~m}): \\
\text { Taxones diferenciales frente a Senecioni-Alnetum }\end{array}$} \\
\hline Valeriana pyrenaica & + & + & + & 1 & 3 & 1 & 1 & + & . & 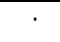 & $\cdot$ & . & . & . & . & 1 & & 1 & $\cdot$ & 1 & 1 & 1 & + & · \\
\hline Chaerophyllum hirsutum & 1 & . & . & + & 2 & 3 & 1 & . & 1 & + & + & + & . & . & . & 1 & 1 & 2 & . & 3 & 2 & 3 & 1 & $\cdot$ \\
\hline Festuca gigantea & & & + & . & 1 & + & . & . & · & . & + & . & . & . & . & & + & $\cdot$ & . & . & + & 1 & . & $\cdot$ \\
\hline Avenella flexuosa & . & + & . & . & . & . & . & . & + & . & . & . & . & . & + & $\cdot$ & . & . & + & . & . & . & . & $\cdot$ \\
\hline Lastrea limbosperma & . & . & . & . & . & . & . & . & · & . & . & . & + & + & 1 & . & . & $\cdot$ & . & . & . & . & . & $\cdot$ \\
\hline Adenostyles alpina & 1 & . & . & . & . & . & . & . & . & . & . & . & . & . & . & . & . & . & . & . & . & . & . & 1 \\
\hline Pimpinella major & . & . & $r$ & . & . & . & . & . & . & . & . & . & . & . & . & + & . & $\cdot$ & . & . & . & . & . & . \\
\hline Helleborus foetidus & . & . & + & . & . & . & . & . & . & . & . & . & . & . & . & + & . & . & . & - & . & . & . & . \\
\hline Erica mackaiana & & . & . & . & . & . & . & - & $\cdot$ & . & . & . & . & . & 1 & & & - & . & . & . & - & . & . \\
\hline Cardamine raphanifolia & & . & . & . & . & . & + & . & . & . & . & . & . & . & $\cdot$ & & & . & . & . & . & . & . & . \\
\hline Cytisus commutatus & & . & . & . & 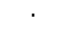 & . & . & $\cdot$ & . & . & . & $\cdot$ & . & . & $\cdot$ & & & $\cdot$ & . & . & . & . & . & + \\
\hline \multicolumn{25}{|c|}{ Taxones diferenciales frente a Hyperico-Alnetum } \\
\hline Hedera hibernica & 3 & 1 & 1 & 1 & 2 & 2 & 2 & 1 & 3 & 2 & 2 & 1 & 2 & 2 & 2 & 1 & 1 & 2 & 1 & 2 & 1 & 1 & 2 & 3 \\
\hline Osmunda regalis & + & 1 & 2 & . & . & ${ }^{\circ}$ & + & . & . & + & 3 & 2 & 1 & 3 & 1 & & 1 & - & 2 & . & . & . & 1 & 1 \\
\hline Betula pubescens & 1 & . & . & . & 1 & 1 & 1 & . & 1 & . & . & 1 & . & 1 & . & 1 & 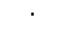 & . & $r$ & . & . & . & 1 & 1 \\
\hline Saxifraga spathularis & 2 & . & + & . & - & . & . & . &. & + & . & + & & 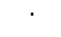 & 1 & & & + & 1 & . & + & . & + & 1 \\
\hline teriana & & . & 1 & . & . & . & . & . & 1 & 3 & + & . & + & . & . & + & + & $\cdot$ & . & . & . & . & . & 1 \\
\hline Viola palustris & 1 & . & . & . & . & . & . & . & . & . & 1 & . & + & 1 & 2 & 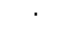 & 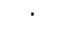 & . & 1 & . & . & . & . & 2 \\
\hline Omphalodes nitida & & . & . & . & . & . & . & . & 1 & + & . & + & . & + & + & & + & $\cdot$ & . & . & . & . & + & $\cdot$ \\
\hline Deschampsia subtriflora & & . & . & . & . & + & . & . & 1 & + & . & . & + & . & $\cdot$ & & 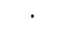 & . & 1 & . & . & . & . & 2 \\
\hline Angelica major & 1 & . & . & + & . & + & . & . & r & 1 & . & . & . & + & . & . & . & . & . & . & . & . & . & . \\
\hline Scrophularia alpestris & 1 & . & . & . & . & . & . & + & . & . & . & . & . & . & . & . & . & . & . & 1 & + & . & . & + \\
\hline Solidago virgaurea & & . & . & . & . & . & . & . & . & . & . & 1 & + & + & + & . & . & $\cdot$ & . & . & . & . & . & . \\
\hline Narcissus asturiensis & . & . & . & . & . & . & . & . & . & . & . & . & 1 & + & $\cdot$ & 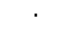 & + & . & 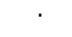 & - & . & . & . & . \\
\hline Hieracium umbellatum & & . & . & . & + & . & . & $\cdot$ & $r$ & $\cdot$ & . & $\cdot$ & . & . & $\cdot$ & & 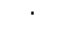 & $\cdot$ & + & . & . & . & . & $\cdot$ \\
\hline Pyrus cordata & & . & $\cdot$ & . & . & 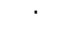 & . & $\cdot$ & $\cdot$ & $\cdot$ & $\cdot$ & + & . & . & + & & . & $\cdot$ & $\mathrm{r}$ & . & . & . & . & $\cdot$ \\
\hline Conopodium majus & & - & $\cdot$ & . & . & 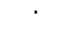 & . & - & $\cdot$ & $\cdot$ & $\cdot$ & $\cdot$ & · & + & - & & 1 & $\cdot$ & - & $\cdot$ & 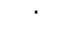 & . & · & $\cdot$ \\
\hline Carex laevigata & 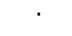 & . & . & . & + & . & . & . & $\cdot$ & . & . & . & 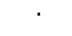 & . & + & & . & . & . & . & 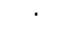 & . & . & $\cdot$ \\
\hline Allium victorialis & & . & $\cdot$ & . & . & . & . & . & . & . & . & . & . & . & . & . & & $\cdot$ & . & . & . & . & . & 2 \\
\hline Arbutus unedo & & · & $\cdot$ & $\cdot$ & $\cdot$ & $\cdot$ & . & $\cdot$ & · & · & · & · & · & $\cdot$ & 1 & · & 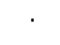 & $\cdot$ & . & . & . & . & . & $\cdot$ \\
\hline Caltha palustris & . & . & . & + & . & . & . & . & . & . & . & . & . & . & . & . & 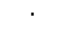 & . & 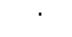 & . & . & . & . & . \\
\hline Vaccinium myrtillus & & & . & . & . & . & . & . & · & . & . & . & . & . & + & & & $\therefore$ & . & . & & . & . & . \\
\hline Ceratocapnos claviculata & & . & . & . & . & . & . & . & . & . & . & . & . & . & $\cdot$ & & + & . & . & . & 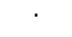 & . & . & . \\
\hline Narcissus triandrus & & - & . & . & . & . & . & . & $\cdot$ & . & . & . & . & . & $\cdot$ & . & + & . & . & . & . & . & . & . \\
\hline Physospermum cornubiense & & r & . & 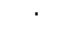 & . & . & . & $\cdot$ & . & $\cdot$ & . & $r$ & . & . & $\cdot$ & . & 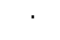 & $\cdot$ & . & . & . & . & r & $\cdot$ \\
\hline
\end{tabular}




\begin{tabular}{|c|c|c|c|c|c|c|c|c|c|c|c|c|c|c|c|c|c|c|c|c|c|c|c|c|}
\hline $\mathrm{N}^{0}$ de orden & 1 & 2 & 3 & 4 & 5 & 6 & 7 & 8 & 9 & 10 & 11 & 12 & 13 & 14 & 15 & 16 & 17 & 18 & 19 & 20 & 21 & 22 & 23 & 24 \\
\hline \multicolumn{25}{|c|}{ Taxones característicos de asociación y unidades superiores } \\
\hline Athyrium filix-femina & 2 & + & 2 & 3 & 1 & 1 & 1 & + & 2 & 1 & 2 & 1 & 2 & 2 & 3 & 1 & 2 & 2 & 1 & 4 & + & 1 & 1 & 2 \\
\hline Dryopteris affinis & 2 & + & 1 & + & 1 & 1 & 1 & 1 & 1 & 2 & . & $\cdot$ & 1 & 1 & 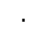 & 1 & 1 & + & + & 1 & 1 & 1 & + & 1 \\
\hline Viola riviniana & + & + & + & + & + & . & + & - & 1 & 1 & 1 & + & 1 & + & 1 & 1 & 1 & + & + & . & . & . & + & 1 \\
\hline Blechnum spicant & 1 & + & + & $\cdot$ & + & . & 1 & . & 1 & + & . & 1 & 1 & 1 & 1 & + & + & 1 & 1 & . & + & . & + & 2 \\
\hline Oxalis acetosella & 1 & . & + & . & + & . & + & . & . & + & + & 1 & + & 1 & 1 & . & 1 & 1 & $\cdot$ & $\cdot$ & 1 & 1 & 1 & 1 \\
\hline Euphorbia dulcis & 1 & 1 & 1 & · & . & . & . & 1 & 2 & + & + & + & 1 & 1 & 1 & . & 1 & 1 & + & $\cdot$ & $\cdot$ & $\cdot$ & $\cdot$ & 1 \\
\hline Hypericum androsaemum & + & r & + & . & . & . & + & . & . & + & 1 & . & . & + & + & + & + & + & . & 1 & + & 1 & + & $\cdot$ \\
\hline Polystichum setiferum & . & 1 & 1 & . & 1 & 1 & 2 & 1 & . & . & . & . & + & . & . & 2 & 1 & 2 & . & 1 & 1 & 3 & 1 & . \\
\hline Brachypodium sylvaticum & + & 1 & + & . & . & + & 1 & . & . & + & . & 2 & . & $\cdot$ & $\cdot$ & 2 & + & 1 & 1 & + & . & 1 & + & $\cdot$ \\
\hline Primula acaulis & + & + & . & . & . & 1 & 1 & + & 1 & 1 & . & . & + & + & . & . & 1 & . & . & . & 1 & + & + & - \\
\hline Ranunculus ficaria & . & 1 & $\cdot$ & . & + & 1 & 1 & . & - & . & 1 & $\cdot$ & + & + & $\cdot$ & . & 2 & 1 & $\cdot$ & . & + & 1 & 1 & \\
\hline Lysimachia nemorum & 1 & + & + & . & 1 & 1 & . & + & 1 & . & + & . & . & + & . & . & . & + & . & . & . & + & + & . \\
\hline Senecio nemorensis & 1 & + & 1 & + & + & 1 & . & . & 2 & 1 & . & + & . & . & . & . & . & . & . & . & . & . & . & 1 \\
\hline Carex remota & . & + & . & . & 2 & . & 1 & . & . & + & . & $\cdot$ & . & . & $\cdot$ & + & + & + & . & $\cdot$ & + & + & . & \\
\hline Phyllitis scolopendrium & . & . & · & . & . & + & 1 & 1 & . & . & . & . & . & $\cdot$ & . & 1 & . & 1 & . & . & 1 & + & 1 & \\
\hline Myosotis martini & . & + & . & 1 & 1 & 1 & . & 1 & 1 & . & . & . & . & $\cdot$ & $\cdot$ & . & 1 & . & . & . & . & . & + & 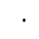 \\
\hline Mercurialis perennis & . & 1 & . & . & . & . & + & 1 & . & . & . & $r$ & . & . & . & . & 1 & 1 & . & . & 1 & + & . & 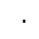 \\
\hline Arum italicum & - & . & . & . & . & . & . & + & . & . & 1 & $\cdot$ & . & $\cdot$ & $\cdot$ & + & + & + & . & $\cdot$ & 1 & 1 & + & \\
\hline Carex pendula & . & . & . & . & . & . & 1 & . & 1 & . & . & . & . & . & $\cdot$ & . & 1 & 2 & . & . & + & + & 1 & \\
\hline Symphytum tuberosum & - & + & . & · & . & . & . & . & . & . & + & + & . & $\cdot$ & $\cdot$ & . & 3 & 1 & . & . & . & . & + & \\
\hline Luzula sylvatica & . & . & . & 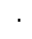 & . & . & . & . & . & . & . & + & . & + & + & . & 1 & 1 & . & . & . & . & . & 2 \\
\hline Potentilla sterilis & . & + & . & 1 & . & . & . & 1 & + & + & . & . & . & . & . & + & . & . & . & . & . & . & . & . \\
\hline Veronica montana & . & . & . & . & + & . & . & . & . & . & 1 & $\cdot$ & . & $\cdot$ & $\cdot$ & 1 & 1 & + & . & . & . & . & . & 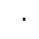 \\
\hline Stachys sylvatica & . & . & . & 1 & . & . & + & . & + & + & . & $\cdot$ & + & $\cdot$ & $\cdot$ & · & . & . & . & . & . & . & . & . \\
\hline Solanum dulcamara & - & . & . & + & + & . & + & + & . & . & $\cdot$ & $\cdot$ & . & $\cdot$ & $\cdot$ & $\cdot$ & . & . & . & $\cdot$ & . & . & . & $\cdot$ \\
\hline Carex sylvatica & . & . & + & ${ }^{\circ}$ & . & . & . & . & . & + & . & . & . & . & $\cdot$ & . & . & + & . & . & + & . & . & \\
\hline Ranunculus tuberosus & . & $r$ & . & . & . & 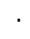 & . & . & . & . & . & . & . & $\cdot$ & + & . & + & . & . & . & + & . & . & \\
\hline
\end{tabular}

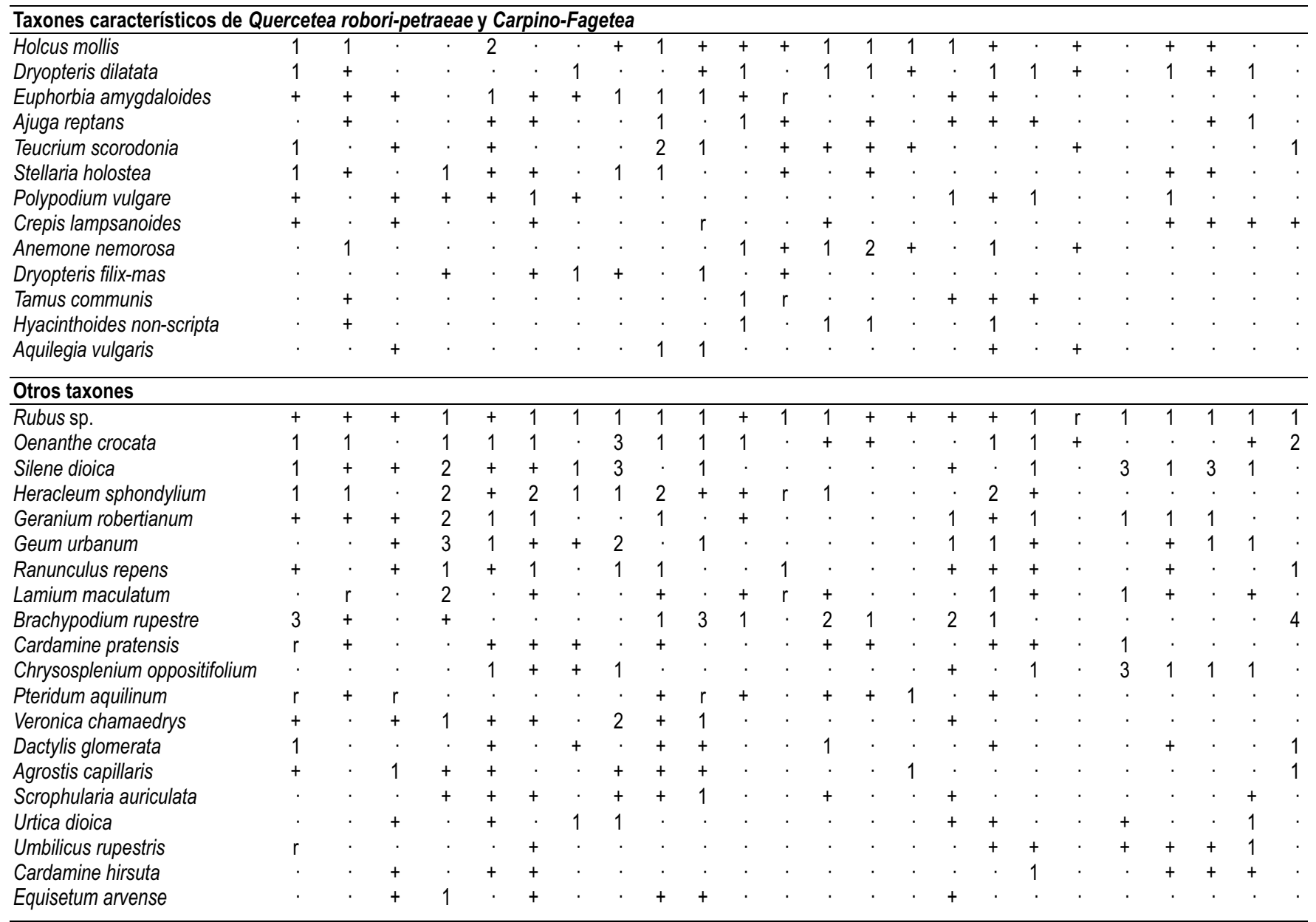




\begin{tabular}{|c|c|c|c|c|c|c|c|c|c|c|c|c|c|c|c|c|c|c|c|c|c|c|c|c|}
\hline $\mathrm{N}^{0}$ de orden & 1 & 2 & 3 & 4 & 5 & 6 & 7 & 8 & 9 & 10 & 11 & 12 & 13 & 14 & 15 & 16 & 17 & 18 & 19 & 20 & 21 & 22 & 23 & 24 \\
\hline Rumex acetosa & $\cdot$ & $\cdot$ & $r$ & $\cdot$ & . & $\cdot$ & $\cdot$ & $\cdot$ & + & . & . & + & + & $\cdot$ & + & $\cdot$ & + & $\cdot$ & $\cdot$ & $\cdot$ & $\cdot$ & $\cdot$ & $\cdot$ & $\cdot$ \\
\hline Filipendula ulmaria & . & + & . & 3 & . & . & $\cdot$ & $\cdot$ & 1 & 1 & . & . & $\cdot$ & $\cdot$ & . & 1 & . & $\cdot$ & $\cdot$ & . & . & . & . & . \\
\hline Lapsana communis & · & $\cdot$ & $\cdot$ & + & + & + & $\cdot$ & $\cdot$ & . & + & . & . & $\cdot$ & $\cdot$ & · & + & $\cdot$ & $\cdot$ & $\cdot$ & $\cdot$ & $\cdot$ & · & $\cdot$ & . \\
\hline Epilobium sp. & . & $\cdot$ & . & $\cdot$ & + & + & . & + & $\cdot$ & $\cdot$ & $\cdot$ & . & $\cdot$ & $\cdot$ & $\cdot$ & + & + & $\cdot$ & $\cdot$ & $\cdot$ & $\cdot$ & . & $\cdot$ & . \\
\hline Anthoxanthum odoratum & . & . & . & . & . & . & . & . & . & . & $\cdot$ & . & 1 & + & 2 & + & $\cdot$ & $\cdot$ & $\cdot$ & $\cdot$ & $\cdot$ & . & . & . \\
\hline Prunella vulgaris & + & $\cdot$ & $\cdot$ & · & + & $\cdot$ & $\cdot$ & · & · & · & · & . & $\cdot$ & $\cdot$ & $\cdot$ & + & · & $\cdot$ & $\cdot$ & $\cdot$ & $\cdot$ & · & $\cdot$ & 1 \\
\hline Glechoma hederacea & · & · & $\cdot$ & · & . & 1 & + & · & . & . & . & . & $\cdot$ & $\cdot$ & $\cdot$ & $\cdot$ & + & $\cdot$ & $\cdot$ & $\cdot$ & $\cdot$ & · & + & $\cdot$ \\
\hline Polypodium cambricum & . & $\cdot$ & $\cdot$ & . & . & + & · & . & . & . & . & . & $\cdot$ & $\cdot$ & $\cdot$ & $\cdot$ & . & + & $\cdot$ & $\cdot$ & + & . & 1 & . \\
\hline Vicia sepium & . & $\cdot$ & + & $\cdot$ & + & · & · & + & + & . & · & · & $\cdot$ & $\cdot$ & $\cdot$ & $\cdot$ & $\cdot$ & $\cdot$ & $\cdot$ & $\cdot$ & $\cdot$ & $\cdot$ & $\cdot$ & $\cdot$ \\
\hline Fragaria vesca & $\cdot$ & $\cdot$ & + & · & · & . & . & . & + & · & $\cdot$ & · & $\cdot$ & $\cdot$ & $\cdot$ & + & + & $\cdot$ & $\cdot$ & $\cdot$ & $\cdot$ & · & $\cdot$ & . \\
\hline Cardamine flexuosa & . & . & . & + & . & . & · & + & + & . & . & . & $\cdot$ & $\cdot$ & . & + & $\cdot$ & $\cdot$ & $\cdot$ & $\cdot$ & $\cdot$ & . & $\cdot$ & . \\
\hline Pentaglottis sempervirens & . & . & . & . & . & + & . & + & . & . & . & . & $\cdot$ & $\cdot$ & . & + & . & $\cdot$ & $\cdot$ & $\cdot$ & $\cdot$ & r & $\cdot$ & . \\
\hline Eupatorium cannabinum & · & . & . & . & . & $\cdot$ & . & . & + & + & . & . & $\cdot$ & $\cdot$ & . & + & r & $\cdot$ & $\cdot$ & $\cdot$ & $\cdot$ & $\cdot$ & $\cdot$ & . \\
\hline Taraxacum gr. officinale & $\cdot$ & $\cdot$ & $\cdot$ & $\cdot$ & . & $\cdot$ & $r$ & · & · & $\cdot$ & . & · & + & $\cdot$ & · & + & $\cdot$ & $\cdot$ & $\cdot$ & $\cdot$ & $\cdot$ & $r$ & $\cdot$ & · \\
\hline Digitalis purpurea & $r$ & $\cdot$ & $r$ & 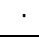 & . & $\cdot$ & $\cdot$ & · & $\cdot$ & . & $\cdot$ & $\cdot$ & + & $\cdot$ & $r$ & $\cdot$ & . & $\cdot$ & $\cdot$ & $\cdot$ & $\cdot$ & $\cdot$ & $\cdot$ & . \\
\hline
\end{tabular}

Taxones presentes en menos de cuatro inventarios: $\mathbf{E}_{\mathbf{1}}+\mathrm{E}_{\mathbf{2}}$ : Acacia melanoxylon: 1 en 13; Eucalyptus globulus: 1 en 11; Juglans regia: + en 7, r en 9 y + en 10; Ligustrum ovalifolium: r en 2; Prunus spinosa: + en 12; Quercus pyrenaica: 1 en 12. Taxones característicos de asociación y unidades superiores: Humulus lupulus: + en 8 y + en 17; Melica uniflora: 1 en 16; Moehringia trinervia: + en 5, + en 11 y + en 16; Pulmonaria longifolia: + en 2, 1 en 12 y 1 en 17. Taxones característicos de Quercetea robori-petraeae y Carpino-Fagetea: Hypericum pulchrum: + en 15; Poa nemoralis: + en 10, + en 14 y + en 16; Stachys officinalis: + en 6, 2 en12 y 1 en 24. Otros taxones: Alliaria petiolata: + en 7, + en 16 y + en 22; Allium triquetrum: + en 11; Angelica sylvestris: + en 16 y + en 23; Anthoxanthum amarum: + en 1; Apium nodiflorum: + en 5; Arrhenatherum bulbosum:1 en 1 y + en 16; Asphodelus lusitanicus: + en 14; Asplenium trichomanes: + en 6 y + en 16; Calystegia sepium: + en 2; Carex lusitanica: + en 4 y r en 9; Carex pilulifera: + en 9; Carex sp.: + en 16; Centaurea rivularis: + en 12 y + en 24; Clematis vitalba: + en 23; Crocus serotinus: + en 10 y + en 14; Cruciata glabra: 1 en 24; Cystopteris fragilis: 1 en 21 y + en 23; Daboecia cantabrica: 1 en 15 y r en 19; Erythronium dens-canis: r en 19; Galium aparine: + en 8, + en 11 y + en 22 ; Galium mollugo: + en 3; Galium palustre: 1 en 4; Galium papillosum: + en 16; Galium sp.: + en 9 y + en 10; Geranium lucidum: 1 en 16; Glyceria fluitans: + en 8; Holcus lanatus: + en 5 y + en 13; Hypericum tetrapterum: + en 5 y + en 10; Limniris pseudacorus: + en 4 y + en 17; Linaria triornithophora: + en 5; Lotus corniculatus: + en 5; Lycopus europaeus: + en 2, + en 3 y + en 9; Lythrum salicaria: + en 10; Molinia caerulea: + en 15 y + en 19; Picris hieracioides: + en 17 y + en 19; Poa pratensis: 1 en 16; Polygonatum odoratum: + en 17; Polygonum hydropiper: + en 4; Polygonum persicaria: + en 5; Polypodium sp.: r en 3; Potentilla erecta: + en 14, + en 15 y r en 19; Prunella grandiflora: + en 10; Pseudoarrhenatherum longifolium: + en 12; Rosa sp. (pl.): 1 en 16; Rubia peregrina: + en 23; Rumex obtusifolius: + en 6 y + en 17; Ruscus aculeatus: r en12, + en 17 y + en 18; Senecio aquaticus: + en 17; Sibthorpia europea: + en 13; Stellaria alsine: + en 5; Stellaria media: + en 6, + en 11 y + en 17; Succisa pratensis: + en 15 y + en 19; Tradescantia fluminensis: 1 en 11, 2 en 17 y 1 en 23; Trifolium pratense: + en 16; Tritonia x crocosmiflora: 1 en 11 y 1 en 17; Ulex europaeus: + en 13 y + en 14; Valeriana dioica: + en 13 y + en 19; Vinca sp.: + en 17.

\section{Procedencia de los inventarios:}

1: Lu: Abadín, Corvite, Río Anllo (621/4796); 2: Lu: Alfoz, A Pontenova, Río Ouro (627/4821); 3: Lu: Baleira, Cortevella, Río Eo (644/4776); 4: Lu: Baleira, entre Airexe y Quintá, Río do Val Pedroso (644/4760); 5: Lu: Baleira, entre O Real y Sampaio, Río Eo (644/4770); 6: Lu: Baleira, Fonteo, aguas debajo de la unión con el Rego do Lameirón (641/4769); 7: Lu: Baleira, Martín, Río de Martín, aguas abajo del puente de Cortevella (643/4776); 8: Lu: Baralla, Río de Guimarei, entre Teixeira y Vilachambre (641/4753); 9: Lu: Baralla, Río Neira, aguas abajo de la piscifactoría de Os Mazos (646/4755); 10: Lu: Baralla, Río Neira, aguas arriba de la potabilizadora de aguas de Baralla (643/4751); 11: Lu: Cervo, A Rueta, Río Xunco (628/4837); 12: Lu: Cervo, Castelo, Río Cobo (625/4835); 13: Lu: Cervo, entre O Covelo y A Estibada, Río Covo (624/4834); 14: Lu: Cervo, entre Vilaestrofe y Rúa, Río Xunco (628/4831); 15: Lu: Cervo, Río Xunco, aguas arriba de As Coruxeiras (627/4829); 16: Lu: Folgoso do Courel, Seoane, Río Lor (652/4722); 17: Lu: Foz, Fazouro, Río Ouro, entre As Granxas y Fontao (634/4826); 18: Lu: Lourenzá, San Tomé de Lourenzá, Río de Baus, aguas arriba de O Cal (637/4809); 19: Lu: Mondoñedo, A Coutada, Río de Figueiras (630/4814); 20: Lu: Mondoñedo, entre Samordás y Bicos (629/4805); 21: Lu: Mondoñedo, Rego da Pedra, entre Lousada y O Galgao (628/4804); 22: Lu: Mondoñedo, Rego do Carballo, entre Lousada y Samordás (629/4804); 23: Lu: Mondoñedo, Río de Valiñadares, entre O Barral y A Fraga do Rei (632/4807); 24: Lu: Mondoñedo, Os Remedios, Estelo, Río do Porto da Cal (627/4810). 
Tabla IVd. Alisedas cantábricas occidentales (Valeriano pyrenaicae-Alnetum glutinosae) variante típica, subvariante típica (cont.)

\begin{tabular}{|c|c|c|c|c|c|c|c|c|c|c|c|c|c|c|c|c|c|c|c|c|c|c|c|c|}
\hline$l^{\circ}$ de orden & 1 & 2 & 3 & 4 & 5 & 6 & 7 & 8 & 9 & 10 & 11 & 12 & 13 & 14 & 15 & 16 & 17 & 18 & 19 & 20 & 21 & 22 & 23 & 24 \\
\hline Altitud (m) & 150 & 55 & 345 & 170 & 55 & 230 & 175 & 275 & 200 & 320 & 195 & 275 & 395 & 205 & 465 & 365 & 180 & 610 & 657 & 5 & 20 & 180 & 3 & 150 \\
\hline endiente $\left({ }^{\circ}\right)$ & 10 & $<2$ & 4 & $<2$ & 2 & 2 & 2 & 2 & 2 & 6 & 2 & 2 & 4 & 6 & 6 & 4 & 4 & $<2$ & 4 & $<2$ & 2 & 2 & $<2$ & 10 \\
\hline rientación & ESE & SW & W & NW & $\mathrm{NE}$ & NW & NW & $\mathrm{N}$ & NNE & $\mathrm{N}$ & NW & S & $E$ & W & NNW & $\mathrm{NE}$ & NNE & SW & NNE & NNE & SE & NW & SSW & SW \\
\hline Itura de copas (m) & 14 & 20 & 20 & 16 & 15 & 14 & 16 & 18 & 18 & 12 & 16 & 20 & 18 & 16 & 16 & 14 & 10 & 22 & 22 & 18 & 22 & 20 & 18 & $6-10$ \\
\hline Cob. $E_{1}(\%)$ & 75 & 100 & 100 & 100 & 100 & 90 & 90 & 100 & 100 & 90 & 100 & 90 & 100 & 100 & 90 & 100 & 85 & 95 & 100 & 90 & 95 & 100 & 100 & 40 \\
\hline Cob. $E_{2}(\%)$ & --- & 25 & $<5$ & 15 & 20 & 20 & 20 & 10 & 20 & 25 & 5 & 25 & 30 & 20 & 15 & 15 & 30 & 20 & 30 & 15 & 20 & 5 & 20 & 20 \\
\hline Cob. & 45 & 90 & 70 & 95 & 95 & 90 & 85 & 90 & 90 & 85 & 90 & 80 & 85 & 95 & 90 & 95 & 95 & 90 & 90 & 95 & 80 & 70 & 90 & 80 \\
\hline Área & 300 & 500 & 300 & 200 & 400 & 300 & 200 & 300 & 400 & 300 & 300 & 300 & 300 & 300 & 300 & 240 & 400 & 400 & 400 & 300 & 300 & 100 & 500 & 190 \\
\hline$N^{0}$ de taxones & 31 & 65 & 48 & 53 & 61 & 56 & 54 & 55 & 51 & 50 & 58 & 58 & 61 & 50 & 42 & 37 & 59 & 60 & 48 & 55 & 56 & 22 & 54 & 23 \\
\hline \multicolumn{25}{|l|}{$E_{1}(>4,0 m)+E_{2}(>$} \\
\hline Alnus & 3 & 4 & 5 & 4 & 5 & 4 & 5 & 4 & 5 & 4 & 5 & 5 & 4 & 3 & 4 & 4 & 4 & 5 & 3 & 5 & 4 & 5 & 5 & 1 \\
\hline Salix & 3 & + & . & 1 & + & 2 & 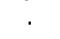 & 2 & 1 & 2 & 1 & 1 & 1 & 1 & 2 & 2 & 2 & + & 1 & 1 & & . & 1 & 1 \\
\hline Fraxi & 1 & 2 & . & 2 & 1 & 1 & 1 & 1 & 1 & 1 & 1 & 1 & 2 & 4 & . & . & 2 & 2 & 4 & + & 1 & . & . & . \\
\hline Coryl & . & 1 & 1 & & 1 & 1 & . & 2 & . & + & . & 1 & 2 & 1 & 2 & 1 & 1 & + & 3 & + & 1 & . & . & 2 \\
\hline Laur & . & 2 & + & 1 & 1 & 2 & 1 & . & 2 & . & + & + & . & + & . & . & 1 & r. & . & 2 & 1 & 1 & 1 & 2 \\
\hline Querc & 1 & + & 1 & 1 & 1 & 1 & 1 & 1 & 1 & . & + & 1 & 1 & . & . & . & 1 & . & . & + & . & . & . & + \\
\hline Cratae & 1 & & . & 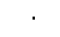 & 1 & 1 & 1 & + & 2 & 1 & + & 1 & 1 & . & . & . & . & 1 & 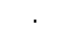 & . & . & 1 & + & . \\
\hline Samb & & + & . & . & . & . & . & 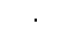 & . & + & . & . & . & 1 & 1 & 1 & 1 & 1 & 2 & 1 & . & 1 & 1 & r \\
\hline Acer & 2 & 2 & . & . & . & 1 & . & + & 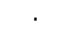 & + & + & 1 & . & . & . & . & . & 1 & . & 2 & 2 & 1 & . & . \\
\hline Casta & . & $r$ & . & . & . & + & . & + & + & 1 & 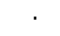 & $\cdot$ & 1 & . & . & . & 1 & + & . & . & . & . & 1 & . \\
\hline Frangula alnus & 1 & & + & . & . & . & $r$ & + & 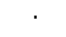 & + & . & r & + & 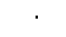 & . & . & . & . & . & . & . & . & . & + \\
\hline Rosa gr. canina & 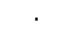 & + & . & & + & 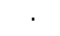 & + & 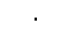 & . & + & 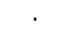 & . & + & . & . & . & & . & + & . & + & . & . & 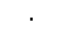 \\
\hline Ilex aquifolium & & & + & & & + & & + & 1 & & 1 & . & + & 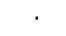 & 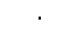 & 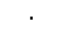 & . & 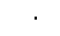 & ${ }^{\circ}$ & . & . & . & 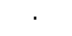 & . \\
\hline Erica arborea & $r$ & & + & & 1 & & & + & . & + & . & . & . & . & 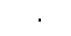 & . & & & . & . & . & . & 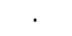 & . \\
\hline
\end{tabular}

\section{$\mathrm{E}_{3}(<1,5 \mathrm{~m})$ :}

Taxones diferenciales frente a Senecioni-Alnetum

Chaerophyllum hirsutum

Valeriana pyrenaica

Festuca gigantea

Avenella flexuosa

Helleborus foetidus

Lastrea limbosperma

Erica vagans

Cytisus commutatus

Saxifraga lepismigena

Scrophularia alpestris

Quercus petraea

Narcissus pseudonarcissus s.l.

\begin{tabular}{|c|c|c|c|c|c|c|c|c|c|c|c|c|c|c|c|c|c|c|c|c|c|c|c|c|}
\hline xor & & & & & & & & & & & & & & & & & & & & & & & & \\
\hline Hedera hibernica & 1 & 3 & 1 & 1 & 2 & 1 & 2 & 2 & 3 & 2 & 3 & 2 & 2 & 2 & 1 & 1 & 2 & 3 & 2 & 2 & 3 & 2 & 2 & 2 \\
\hline Viola riviniana & 1 & 1 & 1 & + & 1 & 1 & + & 1 & 1 & + & + & 1 & + & + & + & . & + & 1 & + & 1 & 1 & + & 1 & . \\
\hline Osmunda regalis & 2 & . & 1 & 2 & 2 & 3 & 2 & 2 & 3 & 1 & 2 & 2 & + & . & . & . & . & . & . & 1 & 2 & 1 & 1 & . \\
\hline Saxifraga spathularis & 1 & + & 1 & 1 & 1 & 1 & 1 & 2 & 1 & + & $r$ & . & + & . & 1 & . & . & . & . & . & 1 & 2 & . & . \\
\hline Carex reuteriana & . & + & 1 & . & 1 & 1 & + & 1 & 1 & . & 1 & 1 & + & . & . & . & . & . & . & . & + & . & . & $\theta$ \\
\hline Omphalodes nitida & . & . & . & . & + & . & $r$ & . & + & + & . & . & + & + & . & . & + & + & . & . & $\mathrm{r}$ & . & + & + \\
\hline Deschampsia subtriflora & 1 & + & . & . & + & 1 & + & 2 & 1 & + & . & . & . & . & . & . & . & . & . & . & $\mathrm{r}$ & 1 & . & . \\
\hline Betula pubescens & . & . & 1 & 1 & 1 & . & . & 1 & 1 & 1 & . & . & 1 & . & . & . & . & . & . & . & . & . & . & + \\
\hline Conopodium majus & . & 1 & 1 & . & 1 & 1 & 1 & + & . & . & . & . & . & . & . & . & . & . & . & + & + & . & . & . \\
\hline Solidago virgaurea & . & . & + & . & + & + & . & + & . & . & . & . & . & . & . & . & . & + & . & . & $\mathrm{r}$ & + & . & . \\
\hline Viola palustris & . & . & 1 & . & . & + & . & + & . & + & . & . & . & . & . & . & . & . & . & . & + & 1 & . & 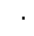 \\
\hline Narcissus triandrus & . & . & + & . & + & 1 & + & + & . & . & . & . & . & . & . & . & . & . & . & . & + & . & . & . \\
\hline Pyrus cordata & 1 & . & + & . & . & . & . & + & . & . & . & . & 1 & . & . & . & . & . & . & . & . & + & . & . \\
\hline Narcissus asturiensis & . & $\cdot$ & + & . & . & + & + & + & $\cdot$ & . & . & . & . & . & . & . & . & . & . & . & . & . & . & · \\
\hline Carex laevigata & . & . & . & 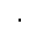 & . & . & . & . & . & + & . & . & . & . & + & + & . & . & . & . & . & . & . & · \\
\hline Caltha palustris & . & . & 1 & . & . & . & . & 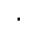 & . & . & . & . & . & 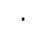 & + & . & . & . & . & . & . & . & . & . \\
\hline Vaccinium myrtillus & . & . & + & . & . & . & . & + & . & . & . & . & . & . & . & . & . & . & . & . & . & . & . & . \\
\hline Dryopteris aemula & . & . & + & . & . & . & . & + & . & . & . & . & . & 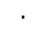 & . & . & . & . & . & . & . & . & . & . \\
\hline Arbutus unedo & + & . & . & . & . & . & . & . & . & . & . & . & . & . & . & . & . & . & . & . & . & . & . & . \\
\hline Physospermum cornubiense & . & . & + & . & . & . & . & . & . & . & . & . & . & . & . & . & . & . & . & . & . & . & . & . \\
\hline Allium victorialis & . & . & . & . & . & . & . & + & & . & . & . & . & . & . & . & . & . & . & . & . & . & . & . \\
\hline
\end{tabular}




\begin{tabular}{|c|c|c|c|c|c|c|c|c|c|c|c|c|c|c|c|c|c|c|c|c|c|c|c|c|}
\hline $\mathrm{N}^{0}$ de orden & 1 & 2 & 3 & 4 & 5 & 6 & 7 & 8 & 9 & 10 & 11 & 12 & 13 & 14 & 15 & 16 & 17 & 18 & 19 & 20 & 21 & 22 & 23 & 24 \\
\hline Hieracium umbellatum & & & & & & & & & & & & + & & & & & & & & & & & & \\
\hline Angelica major & & & & & & & & & & & & 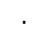 & . & . & . & . & & + & . & & & & . & \\
\hline \multicolumn{25}{|c|}{ Taxones característicos de asociación y unidades superiores } \\
\hline Athyrium filix-femina & + & 3 & 1 & 1 & 1 & 1 & 1 & 1 & 1 & 2 & 1 & 2 & 2 & 1 & 1 & + & + & 1 & 1 & 1 & 1 & 4 & 2 & 2 \\
\hline Dryopteris affinis & 1 & 1 & 2 & 1 & 1 & 2 & 1 & 1 & 1 & 1 & 1 & 1 & 2 & 1 & 2 & 2 & + & . & 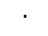 & + & + & 1 & 1 & 1 \\
\hline Lonicera hispanica & 1 & 1 & 1 & 1 & 1 & 1 & 1 & 1 & 1 & 1 & . & 1 & 1 & 1 & 1 & . & 1 & + & 1 & 1 & 1 & 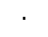 & 1 & + \\
\hline Blechnum spicant & 1 & + & 1 & + & 1 & 1 & + & 1 & 1 & 1 & + & + & 1 & 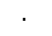 & 1 & . & + & . & . & 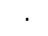 & + & 1 & + & 2 \\
\hline Oxalis acetosella & . & 1 & + & 1 & . & 1 & + & + & 1 & + & + & + & 1 & 1 & 1 & . & + & 1 & . & 1 & + & . & 1 & 1 \\
\hline Primula acaulis & . & + & . & + & 1 & + & + & + & & 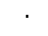 & 1 & + & + & + & + & + & 1 & + & + & + & + & . & . & . \\
\hline Brachypodium sylvaticum & 1 & 1 & . & 1 & + & . & 3 & & . & 1 & 1 & 1 & 1 & 1 & . & . & . & 1 & + & 1 & 2 & 1 & 2 & . \\
\hline Euphorbia dulcis & . & 1 & 1 & + & 1 & 1 & 1 & 1 & 1 & + & . & . & . & 1 & . & . & + & 1 & . & 1 & 1 & 1 & 1 & . \\
\hline Luzul & 1 & 1 & $\cdot$ & 1 & 2 & 1 & 1 & + & 1 & 1 & 1 & 1 & 1 & 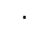 & 1 & . & . & 1 & . & + & . & $\cdot$ & . & . \\
\hline Polystichum setiferum & . & 1 & . & 1 & 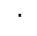 & . & . & . & . & 2 & 1 & 2 & 2 & 1 & 1 & 2 & 3 & 1 & 1 & 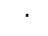 & 1 & . & + & . \\
\hline Ranunculus ficaria & . & 1 & . & 1 & 1 & 1 & 1 & . & 1 & & 1 & 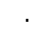 & . & 1 & 1 & . & 1 & 1 & . & 1 & 1 & . & 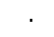 & 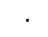 \\
\hline Carex & . & 1 & . & + & . & . & . & . & . & 1 & 1 & . & 1 & + & + & + & + & + & 1 & + & . & . & 1 & . \\
\hline Hypericum androsaemum & . & . & + & + & + & + & . & . & + & & + & + & + & + & . & . & 1 & . & . & . & . & . & + & $\mathrm{r}$ \\
\hline Carex pendula & . & + & + & . & . & . & . & $r$ & + & . & 1 & 1 & + & . & $\cdot$ & . & . & + & . & . & + & . & 2 & 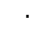 \\
\hline Lysimachia nemorum & . & . & . & + & . & . & . & . & + & 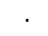 & 1 & 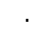 & + & . & + & . & . & 1 & . & 1 & . & + & + & . \\
\hline Arum italicum & . & + & . & . & . & . & . & . & & 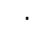 & + & 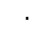 & . & + & $\cdot$ & + & + & . & + & + & + & $\cdot$ & + & . \\
\hline orensis & . & . & . & . & + & + & . & . & + & . & . & + & + & . & $\cdot$ & . & . & . & . & + & . & . & . & 1 \\
\hline opendrium & . & . & . & + & . & . & . & . & & . & . & . & . & 1 & 1 & 2 & 1 & + & 1 & & . & . & . & . \\
\hline Pulmonaria longifolia & . & + & . & + & + & . & + & . & . & 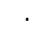 & + & . & + & . & . & . & . & . & . & . & . & . & + & . \\
\hline Symphytum tuberosum & . & 3 & . & . & . & . & . & . & & & + & $\cdot$ & $\cdot$ & 3 & $\cdot$ & . & 1 & $\cdot$ & $\cdot$ & + & + & $\cdot$ & $\cdot$ & . \\
\hline Mercurialis perennis & . & 1 & . & . & . & . & . & . & & 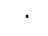 & . & . & $\cdot$ & 3 & 1 & 1 & . & . & . & . & + & . & 1 & . \\
\hline Veronica montana & . & 2 & . & + & . & . & 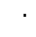 & & & & . & . & + & 1 & . & . & + & 1 & . & $\cdot$ & . & . & . & . \\
\hline Stachys sylvatica & . & + & . & . & . & . & . & . & & . & . & + & . & . & . & . & + & 1 & + & + & . & . & . & . \\
\hline Ranunculus tuberosus & . & . & . & . & 1 & 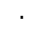 & + & 1 & & 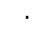 & . & . & + & 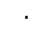 & + & . & . & . & . & . & . & . & . & . \\
\hline Circae & . & + & . & + & . & . & . & . & . & 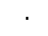 & . & + & . & . & $\cdot$ & . & + & + & . & . & . & . & . & . \\
\hline Cares & . & . & . & . & . & . & . & . & & + & + & + & + & 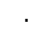 & . & . & . & . & . & . & + & . & . & . \\
\hline camara & & + & . & . & 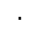 & . & 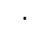 & 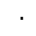 & & & . & . & . & . & . & . & . & + & . & + & . & . & 1 & \\
\hline Potentilla sterilis & . & + & . & . & . & . & . & & & & . & . & + & . & $\cdot$ & + & . & . & . & 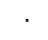 & . & $\cdot$ & + & \\
\hline
\end{tabular}

\section{Taxones característicos de Quercetea robori-petraeae y Carpino-Fagetea}

\begin{tabular}{|c|c|c|c|c|c|c|c|c|c|c|c|c|c|c|c|c|c|c|c|c|c|c|c|c|}
\hline Ajuga reptans & $\cdot$ & 1 & $\cdot$ & + & 1 & + & + & 1 & + & 1 & + & $\cdot$ & 1 & + & + & + & + & 1 & 1 & + & . & $\cdot$ & $\cdot$ & . \\
\hline Dryopteris dilatata & . & 1 & . & 1 & 1 & 1 & + & . & 1 & + & . & . & 1 & + & 1 & 1 & 1 & . & + & 1 & . & . & 1 & + \\
\hline Holcus mollis & + & + & 2 & . & . & 1 & 1 & 2 & 1 & 1 & 1 & 1 & + & . & 1 & . & 1 & 1 & . & + & . & . & + & . \\
\hline Euphorbia amygdaloides & . & + & . & . & . & . & $r$ & + & + & + & 1 & + & + & 1 & . & $r$ & + & 1 & . & + & + & . & + & . \\
\hline Polypodium vulgare & + & $\cdot$ & . & + & 1 & 1 & + & 1 & 1 & 1 & . & + & · & + & + & + & . & . & + & . & . & . & . & . \\
\hline Teucrium scorodonia & . & + & + & + & 1 & . & 1 & . & 1 & 1 & . & . & . & . & . & . & 1 & . & . & 1 & + & . & . & . \\
\hline Anemone nemorosa & . & 1 & . & 1 & 1 & + & 1 & . & + & $\cdot$ & . & . & + & . & . & . & . & . & . & + & 2 & + & . & . \\
\hline Aquilegia vulgaris & . & $\cdot$ & + & $\cdot$ & . & + & + & · & + & + & . & + & + & . & . & . & . & . & $\cdot$ & . & + & . & + & . \\
\hline Stellaria holostea & . & 1 & . & 1 & . & . & + & . & . & + & . & . & 1 & . & 1 & . & 1 & . & + & . & . & . & . & 1 \\
\hline Crepis lampsanoides & . & + & . & + & 1 & 1 & . & . & + & $\cdot$ & . & 1 & . & . & . & . & . & . & . & . & . & . & . & . \\
\hline Tamus communis & . & + & . & . & . & . & . & . & . & . & . & . & + & . & . & . & 1 & . & + & . & . & . & + & . \\
\hline Stachys officinalis & . & $\cdot$ & . & $\cdot$ & + & + & + & . & + & $\cdot$ & . & + & . & . & . & . & . & . & . & . & . & . & . & . \\
\hline Dryopteris filix-mas & . & . & . & $\cdot$ & . & . & . & . & . & . & . & 1 & . & . & 1 & + & . & + & 1 & . & . & · & . & . \\
\hline \multicolumn{25}{|l|}{ Otros taxones } \\
\hline Rubus sp. & 1 & . & 1 & 2 & + & + & . & + & 1 & 1 & + & 1 & + & 1 & 1 & 1 & 1 & + & 1 & 1 & . & + & ${ }^{\circ}$ & + \\
\hline Geranium robertianum & . & + & . & 1 & . & . & + & . & . & + & + & + & 1 & 1 & 1 & 1 & 2 & 1 & 1 & . & + & . & + & . \\
\hline Silene dioica & . & 1 & . & 2 & . & . & . & . & . & 1 & + & . & . & 2 & 2 & 3 & 1 & 2 & 2 & . & + & . & + & . \\
\hline Geum urbanum & . & 1 & . & + & . & . & . & . & . & + & 1 & . & + & 1 & $\cdot$ & + & + & 2 & 1 & + & . & . & + & . \\
\hline Ranunculus repens & + & $\cdot$ & + & + & . & . & . & . & . & $\cdot$ & 1 & . & + & . & + & 2 & 1 & 1 & 1 & . & . & . & + & . \\
\hline Lamium maculatum & . & + & . & + & . & . & + & . & . & . & + & . & . & 1 & . & 1 & 1 & . & + & 1 & + & . & + & . \\
\hline Umbilicus rupestris & . & + & $\cdot$ & 1 & + & + & . & . & 1 & $\cdot$ & + & 1 & 1 & $\cdot$ & $\cdot$ & . & + & . & $\cdot$ & + & + & . & . & . \\
\hline Cardamine pratensis & + & . & . & + & . & . & + & . & . & + & . & . & . & + & 1 & + & 1 & . & . & . & + & . & + & . \\
\hline Dactylis glomerata & . & . & . & $\cdot$ & + & . & . & $r$ & + & + & + & . & . & + & . & + & . & + & . & . & + & . & + & . \\
\hline Brachypodium rupestre & . & 1 & + & . & 1 & 1 & . & 1 & 2 & . & 2 & . & + & . & . & . & + & . & . & . & . & . & . & . \\
\hline Angelica sylvestris & . & . & $\cdot$ & 1 & + & . & . & . & $\cdot$ & . & + & $\cdot$ & . & + & 1 & . & 1 & . & $\cdot$ & + & . & . & 1 & . \\
\hline Heracleum sphondylium & . & $\cdot$ & . & $\cdot$ & $\cdot$ & . & . & + & + & . & 1 & . & . & + & . & . & . & 1 & 1 & . & 1 & . & + & . \\
\hline Prunella vulgaris & . & . & + & $\cdot$ & 1 & 1 & . & . & . & . & + & . & . & . & . & . & . & + & . & . & + & . & 1 & . \\
\hline Ruscus aculeatus & . & + & . & + & 1 & . & . & . & . & . & . & + & + & . & + & . & . & . & $\cdot$ & · & + & . & . & · \\
\hline Scrophularia auriculata & . & $\cdot$ & . & $\cdot$ & . & . & . & . & . & . & . & . & + & + & $\cdot$ & + & + & + & 1 & + & . & . & . & . \\
\hline Rumex acetosa & . & + & . & $\cdot$ & + & . & + & . & . & . & + & . & . & . & $\cdot$ & . & + & . & $\cdot$ & + & + & . & . & . \\
\hline Chrysosplenium oppositifolium & . & . & . & . & . & . & . & . & . & . & . & . & 1 & 2 & 3 & 3 & 1 & . & 2 & . & . & . & . & . \\
\hline
\end{tabular}




\begin{tabular}{|c|c|c|c|c|c|c|c|c|c|c|c|c|c|c|c|c|c|c|c|c|c|c|c|c|}
\hline $\mathrm{N}^{0}$ de orden & 1 & 2 & 3 & 4 & 5 & 6 & 7 & 8 & 9 & 10 & 11 & 12 & 13 & 14 & 15 & 16 & 17 & 18 & 19 & 20 & 21 & 22 & 23 & 24 \\
\hline Tritonia x crocosmiflora & $\cdot$ & 1 & . & + & 1 & . & $\cdot$ & $\cdot$ & . & . & . & . & . & $\cdot$ & . & $\cdot$ & + & . & . & 1 & + & . & . & . \\
\hline Urtica dioica & $\cdot$ & . & . & . & · & . & $\cdot$ & . & $\cdot$ & $\cdot$ & . & + & . & . & . & + & 1 & + & 1 & . & . & $\cdot$ & + & . \\
\hline Asphodelus lusitanicus & $\cdot$ & . & . & + & . & + & $r$ & . & + & . & . & . & $\cdot$ & . & . & $\cdot$ & . & . & . & . & + & $\cdot$ & $\cdot$ & + \\
\hline Digitalis purpurea & $r$ & . & . & . & · & · & r & $r$ & + & $\cdot$ & $\cdot$ & r & $\cdot$ & + & $\cdot$ & $\cdot$ & $\cdot$ & $\cdot$ & $\cdot$ & $\cdot$ & · & $\cdot$ & $\cdot$ & · \\
\hline Pteridum aquilinum & $\cdot$ & + & . & + & + & + & · & + & . & $\cdot$ & $\cdot$ & . & $\cdot$ & $\cdot$ & $\cdot$ & $\cdot$ & $\cdot$ & $\cdot$ & $\cdot$ & $\cdot$ & · & $\cdot$ & $\cdot$ & $r$ \\
\hline Pentaglottis sempervirens & $\cdot$ & . & . & $\cdot$ & . & . & . & . & . & $\cdot$ & + & + & $\cdot$ & $\cdot$ & $\cdot$ & $\cdot$ & + & + & + & · & . & $\cdot$ & $\cdot$ & . \\
\hline Galium aparine & $\cdot$ & + & . & $\cdot$ & $\cdot$ & $\cdot$ & r & . & . & $\cdot$ & + & · & $\cdot$ & + & $\cdot$ & $\cdot$ & + & $\cdot$ & $\cdot$ & $\cdot$ & · & $\cdot$ & $\cdot$ & $\cdot$ \\
\hline Taraxacum gr. officinale & $\cdot$ & . & . & . & · & $\cdot$ & . & $\cdot$ & $r$ & $\cdot$ & + & r & $\cdot$ & $\cdot$ & . & $r$ & $\cdot$ & $\cdot$ & . & + & · & $\cdot$ & $\cdot$ & · \\
\hline Cardamine flexuosa & $\cdot$ & . & . & . & . & + & . & $\cdot$ & . & $\cdot$ & $\cdot$ & $\cdot$ & $\cdot$ & $\cdot$ & $\cdot$ & $\cdot$ & 1 & + & 1 & . & . & . & $\cdot$ & . \\
\hline Lapsana communis & $\cdot$ & . & + & $\cdot$ & $\cdot$ & $\cdot$ & + & . & · & $\cdot$ & $\cdot$ & $\cdot$ & $\cdot$ & $\cdot$ & $\cdot$ & $\cdot$ & $\cdot$ & 1 & $\cdot$ & $\cdot$ & + & $\cdot$ & $\cdot$ & $\cdot$ \\
\hline Eupatorium cannabinum & $\cdot$ & . & · & . & $\cdot$ & $\cdot$ & . & · & · & $\cdot$ & $\cdot$ & 1 & $\cdot$ & $\cdot$ & $\cdot$ & $\cdot$ & $\cdot$ & + & + & + & $\cdot$ & $\cdot$ & $\cdot$ & $\cdot$ \\
\hline Limniris pseudacorus & $\cdot$ & . & . & . & . & $\cdot$ & . & . & · & $\cdot$ & $\cdot$ & $\cdot$ & $\cdot$ & $\cdot$ & · & $\cdot$ & + & $\cdot$ & 1 & $\cdot$ & r & $\cdot$ & + & $\cdot$ \\
\hline Picris hieracioides & $\cdot$ & . & . & $\cdot$ & + & $\cdot$ & . & $r$ & . & . & $\cdot$ & $\cdot$ & $\cdot$ & $\cdot$ & $\cdot$ & $\cdot$ & + & $\cdot$ & . & + & $\cdot$ & $\cdot$ & $\cdot$ & . \\
\hline Centaurea rivularis & $\cdot$ & . & $\cdot$ & $\cdot$ & + & $\cdot$ & . & . & $r$ & . & + & $\cdot$ & $\cdot$ & $\cdot$ & $\cdot$ & $\cdot$ & . & $\cdot$ & $\cdot$ & + & $\cdot$ & $\cdot$ & $\cdot$ & . \\
\hline
\end{tabular}

Taxones presentes en menos de cuatro inventarios:

$\mathbf{E}_{1}+\mathbf{E}_{2}$ : Acacia melanoxylon: + en 5 y 1 en 6; Cytisus scoparius: r en 6; Cytisus striatus: + en 1; Eucalyptus globulus: 1 en 6; Genista florida: 1 en 12; Ligustrum ovalifolium: 1 en $2,+$ en 20 y + en 23, Prunus avium: $r$ en 18; Ulmus glabra: 1 en 12 . Taxones característicos de asociación y unidades superiores: Myosotis martini: 1 en 4 y + en 11; Sanicula europea: + en 13. Taxones característicos de Quercetea robori-petraeae y Carpino-Fagetea: Lathyrus linifolius: + en 7, r en 8 y + en 9; Melampyrum pratense: + en 7; Melittis melissophyllum: + en 13; Poa nemoralis: + en 19 y + en 20. Otros taxones: Agrostis capillaris: + en 9, + en 10 y 1 en $18 ;$ Allium triquetrum: 1 en 20; Anthoxanthum amarum: + en 5 y + en 8; Arrhenatherum bulbosum: 1 en 3, 1 en 8 y 1 en 11 ; Briza media: 1 en 23; Calystegia sepium: + en17; Cardamine hirsuta: + en 12, 1 en 14 y + en 17; Carex cf. umbrosa: + en 5; Carex pilulifera: 1 en 3; Clematis vitalba: 1 en 12; Coincya setigera: r en 10; Crocus serotinus: 1 en 6; Cruciata glabra: 1 en 3, + en 8 y + en 13; Daboecia cantabrica: + en 5; Epilobium sp.: r en 7; Equisetum arvense: 1 en 12 y 1 en 18; Filipendula ulmaria: 1 en 23; Fragaria vesca: + en 21; Galeopsis tetrahit: + en 18; Glechoma hederacea: 1 en 2, 1 en 11 y + en 23; Glyceria fluitans: + en 18; Holcus lanatus: + en 6 y + en 16; Juncus effusus: r en 15; Juncus sp.: + en 14; Linaria triornithophora: + en 20; Lotus corniculatus: r en 20; Lycopus europaeus: + en 12; Mentha aquatica: + en 23; Mentha pulegium: 1 en 12; Mentha suaveolens: + en 19; Phalaris arundinacea: + en 2, + en 18 y + en 19 ; Plantago lanceolata: r en 11; Poa trivialis: + en 14; Polygonatum odoratum: + en $21 ;$ Polygonum persicaria: + en 12 y + en 23; Polypodium interjectum: + en 7 y + en 21; Potentilla erecta: r en 3; Potentilla reptans: + en 12; Rumex obtusifolius: + en 11, + en 12 y + en 14; Senecio aquaticus: + en 5; Sonchus oleraceus: + en 12; Tradescantia fluminensis: + en 20; Valeriana dioica: 1 en 23; Veronica chamaedrys: 1 en 2 , + en 18 y + en $19 ;$ Vicia sepium: + en 13 y 1 en 19; Zanthedeschia aethyopica: + en 20.

Procedencia de los inventarios:

1: Lu: Mondoñedo, Río Tronceda, entre A Cabana ye Tronceda de Abaixo (631/4811); 2: Lu: Mondoñedo, Santo André de Masma, margen izquierda del Río Masma (633/4814); 3: Lu: Muras, Rego de Santar, entre Santar de Arriba y Santar de Abaixo (603/4821); 4: Lu: Ourol, Río Grandal, aguas abajo de Lobamorta (610/4824); 5: Lu: Ourol, Río Landro aguas arriba de Atián de Abaixo (612/4827); 6: Lu: Ourol, Río Landro, entre Vilabuín y Atián de Abaixo (613/4822); 7: Lu: Ourol, Río Landro, margen izquierda aguas abajo del puente de Xerdiz (612/4824); 8: Lu: Ourol, Río Sor, aguas arriba de Pena de Mouriscón (601/4823); 9: Lu: Ourol, Souto Chao, Río Xanceda (611/4822); 10: Lu: Ribeira de Piquín, entre Llencias y Envernego, Rego de Valín Salgueiro (649/4781); 11: Lu: Ribeira de Piquín, O Chao, margen derecha del Río Eo (646/4785); 12: Lu: Ribeira de Piquín, Os Baos, Río Rodil (649/4782); 13: Lu: Ribeira de Piquín, Río Lúa, aguas arriba del puente entre Boel y Crende (643/4780); 14: Lu: Riotorto, Rego de Machín, aguas arriba de Piñeiro (639/4803); 15: Lu: Ríotorto, Río de Ferreiras, entre O Marco de Alvare y O Salgairedo (639/4794); 16: Lu: Ríotorto, Río de Ferreiras, entre O Salgairedo y Os Carrís (639/4795); 17: Lu: Riotorto, Río do Carboeiro, entre O Carboerio y As Rodrigas (640/4799); 18: Lu: Samos, Río Oribio, entre Triacastela y San Cristobo do Real (642/4734); 19: Lu: Triacastela, Santalla de Alfoz, Río de Santalla, aguas arriba de Ponte de Matavella (644/4736); 20: Lu: Viveiro, Galdo, San Martiño, Río de Bravos (612/4831); 21: Lu: Viveiro, Souto da Retorta, margen derecha del Río Landro (613/4830); 22: Lu:Viveiro, Valcarría, Rego da Fraga de Loureiro (616/4830), 23: Lu: Barreiros, O Pozo Mouro, Río de Santo Estebo (640/4821), 24: Lu: Barreiros, A Ínsua, margen derecha del Rego dos Trobos (643/4820). 
Tabla V. Alisedas cantábricas occidentales (Valeriano pyrenaicae-Alnetum glutinosae) (cont.) variante típica, subvariante mesotrofa ( ${ }^{*}$ presencia de rocas carbonatadas en el tramo de cauce estudiado)

\begin{tabular}{|c|c|c|c|c|c|c|c|c|c|c|c|c|c|c|c|c|c|c|c|c|c|c|c|c|}
\hline $\mathrm{V}^{0}$ de orden & 1 & $2^{*}$ & $3^{*}$ & 4 & 5 & 6 & 7 & 8 & 9 & $10^{*}$ & $11^{*}$ & $12^{*}$ & 13 & 14 & $15^{*}$ & $16^{*}$ & 17 & 18 & 19 & $20^{*}$ & 21 & 22 & $23^{*}$ & 24 \\
\hline Altitud (m) & 425 & 560 & 470 & 785 & 535 & 45 & 620 & 205 & 95 & 515 & 510 & 410 & 475 & 585 & 535 & 910 & 4003 & 395 & 205 & 320 & 375 & 850 & 630 & 400 \\
\hline Pendiente $\left({ }^{\circ}\right)$ & 2 & 2 & 2 & 6 & 2 & 4 & $<2$ & $<2$ & $<2$ & 4 & 2 & 2 & $<2$ & 4 & 4 & 4 & $<2$ & 2 & 8 & 16 & $<2$ & 10 & $<2$ & $<2$ \\
\hline Orientación & WNW & NNW & SSE & SE & NW & E & E V & WNW & W & NNW & W & W & W & SW $\varsigma$ & SSE & SSW & S & 51 & NNE & NNW & $\mathrm{NE}$ & W & SSE & S \\
\hline Altura de copas (m) & 20 & 16 & 10 & 16 & 24 & 18 & 20 & 14 & 24 & 24 & 16 & 20 & 18 & 28 & 26 & $6-10$ & 26 & 28 & 16 & 18 & 24 & 10 & 18 & 28 \\
\hline Cob. $E_{1}(\%)$ & 100 & 100 & 100 & 100 & 90 & 95 & 100 & 100 & 100 & 100 & 100 & 100 & 100 & 100 & 95 & 100 & 1001 & 100 & 90 & 90 & 100 & 90 & 100 & 100 \\
\hline Cob. $E_{2}(\%)$ & 10 & 25 & 35 & 15 & 5 & 10 & 20 & 25 & 20 & 50 & 30 & 15 & --- & 30 & 15 & 30 & 20 & 15 & 15 & 10 & 20 & 15 & 20 & 20 \\
\hline (\%) & 75 & 90 & 80 & 85 & 90 & 100 & 70 & 50 & 80 & 95 & 100 & 95 & 90 & 85 & 70 & 70 & 90 & 95 & 80 & 70 & 95 & 75 & 85 & 90 \\
\hline Área $\left(m^{2}\right)$ & 300 & 300 & 200 & 300 & 300 & 200 & 500 & 200 & 500 & 400 & 200 & 300 & 500 & 300 & 400 & 300 & 5003 & 300 & 300 & 200 & 400 & 200 & 300 & 400 \\
\hline $\mathrm{N}^{0}$ de taxones & 43 & 52 & 43 & 52 & 48 & 51 & 52 & 45 & 89 & 66 & 57 & 68 & 52 & 51 & 50 & 59 & 84 & 72 & 43 & 25 & 55 & 31 & 67 & 60 \\
\hline \multicolumn{25}{|l|}{$E_{1}(>4,0 m)+E_{2}(>1,5-4,0 r$} \\
\hline Alnus glutinosa & 5 & 5 & 4 & 4 & 5 & 4 & 4 & 4 & 3 & 4 & 4 & 5 & 5 & 5 & 5 & 3 & 5 & 5 & 4 & 5 & 5 & 3 & 4 & $\overline{3}$ \\
\hline Fraxinus excelsior & 1 & 1 & 3 & 2 & 1 & 1 & 2 & 2 & 3 & 2 & 2 & 1 & 1 & 1 & 1 & 3 & 1 & 1 & 2 & 1 & 1 & 1 & 1 & 3 \\
\hline Salix atrocinerea & 1 & 1 & 3 & 1 & 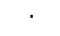 & & 2 & 1 & + & + & & 1 & $r$ & 1 & 1 & 2 & + & + & 2 & & 1 & 2 & 1 & + \\
\hline Corylus avellana & 1 & 2 & . & 1 & 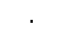 & + & + & 1 & 1 & 2 & . & 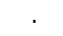 & . & 1 & + & 3 & 1 & + & 1 & 1 & 1 & 2 & + & + \\
\hline Sambucus nigra & 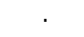 & 1 & 2 & + & + & . & 1 & 1 & + & 1 & 1 & 1 & 1 & 1 & 1 & . & 1 & + & 1 & 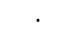 & + & . & 1 & + \\
\hline Acer pseudoplatan & . & 2 & . & 1 & 2 & 1 & 2 & + & 2 & 2 & . & + & $\cdot$ & 2 & 1 & 1 & . & & . & & + & 2 & 2 & 2 \\
\hline Rosa gr. canina & . & 1 & . & . & + & & + & . & + & 1 & . & . & + & + & + & 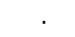 & + & + & . & & . & . & + & 1 \\
\hline Crata & 1 & . & . & . & 1 & 1 & 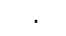 & 1 & & + & + & 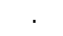 & . & 1 & 1 & . & 1 & & . & & . & . & . & + \\
\hline Castanea sativa & . & . & + & . & 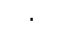 & 1 & . & . & 1 & 1 & 1 & . & $r$ & & . & . & . & $r$ & $r$ & & . & . & + & . \\
\hline Laurus nobilis & . & . & + & + & . & 1 & . & . & . & . & r & . & . & . & . & . & 1 & . & 2 & 1 & + & . & . & . \\
\hline Quercus robur & . & . & . & $r$ & . & 1 & . & . & 1 & . & . & 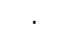 & + & 1 & 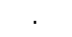 & 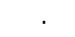 & 1 & + & 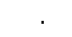 & & 1 & . & . & . \\
\hline Prunus spinosa & . & . & . & . & + & . & 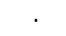 & . & + & 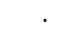 & 1 & . & . & . & . & . & + & & . & & . & . & . & 1 \\
\hline Prunus avium & . & 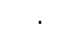 & + & 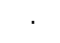 & & + & + & 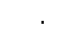 & & + & & & $\cdot$ & & & 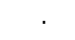 & . & & 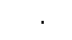 & & . & 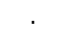 & . & . \\
\hline
\end{tabular}

$\mathrm{E}_{3}(<1,5 \mathrm{~m})$ :

Taxones diferenciales frente a Senecioni-Alnetum

\begin{tabular}{llll}
\hline Chaerophyllum hirsutum & 1 & 3 & 3
\end{tabular}

Valeriana pyrenaica

Festuca gigantea

Helleborus foetidus

Cardamine raphanifolia

Pimpinella major

Scrophularia alpestris

Cardamine impatiens

Campanula adsurgens

Narcissus pseudonarcissus s.l.

Fagus sylvatica

\begin{tabular}{|c|c|c|c|c|c|c|c|c|c|c|c|c|c|c|c|c|c|c|c|c|c|c|c|c|}
\hline \multicolumn{25}{|c|}{ Taxones diferenciales frente a Hyperico-Alnetum } \\
\hline Hedera hibernica & + & 2 & 2 & 1 & + & 2 & + & + & 2 & 3 & 2 & 1 & + & 3 & 2 & 1 & 3 & 2 & 2 & 2 & 3 & 1 & 3 & 4 \\
\hline Viola riviniana & r & 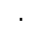 & . & . & + & 1 & . & · & 1 & + & . & 1 & + & $\cdot$ & + & + & 1 & 1 & . & · & 1 & · & + & + \\
\hline Carex reuteriana & . & + & . & + & . & 1 & + & 1 & 1 & 1 & . & 1 & $\cdot$ & $\cdot$ & + & . & 3 & 3 & . & . & 3 & . & . & + \\
\hline Saxifraga spathularis & + & . & . & 1 & . & + & . & . & + & + & . & . & + & 1 & . & . & . & · & . & . & . & 1 & . & · \\
\hline Omphalodes nitida & $\cdot$ & $\cdot$ & . & . & . & + & . & + & 1 & . & + & . & $\cdot$ & $\cdot$ & . & + & + & + & + & . & . & . & . & · \\
\hline Osmunda regalis & 1 & . & . & . & . & 1 & . & 1 & 3 & . & . & . & . & . & . & . & + & + & · & . & + & . & . & · \\
\hline Betula pubescens & . & 1 & . & 1 & . & . & . & . & 1 & . & . & . & . & . & . & . & . & . & . & . & . & 2 & 1 & . \\
\hline Solidago virgaurea & $\cdot$ & $\cdot$ & . & $\cdot$ & · & 1 & . & · & + & . & . & · & $\cdot$ & $\cdot$ & $\cdot$ & $\cdot$ & $\cdot$ & $\cdot$ & $\cdot$ & . & . & . & . & · \\
\hline Erica arborea & . & . & . & + & . & · & . & . & $\cdot$ & . & . & . & $\cdot$ & . & . & . & $\cdot$ & $\cdot$ & . & . & . & 1 & . & $\cdot$ \\
\hline Angelica major & . & . & . & . & . & . & . & . & $\cdot$ & . & . & . & $\cdot$ & . & $\cdot$ & . & . & + & . & . & + & . & . & . \\
\hline Deschampsia subtriflora & . & $\cdot$ & . & . & . & . & . & . & $\cdot$ & 1 & . & · & $\cdot$ & $\cdot$ & . & . & $\cdot$ & $\cdot$ & . & . & · & . & . & $\cdot$ \\
\hline Hieracium umbellatum & . & $\cdot$ & · & . & . & . & . & · & + & . & . & · & $\cdot$ & . & . & . & . & $\cdot$ & . & . & . & . & . & · \\
\hline Pyrus cordata & $\cdot$ & $\cdot$ & . & . & $\cdot$ & . & . & · & $r$ & . & . & . & $\cdot$ & . & . & $\cdot$ & . & $\cdot$ & . & . & · & . & . & · \\
\hline \multicolumn{25}{|c|}{ Taxones diferenciales de subvariante mesotrofa (y dif. frente a Senecioni-Alnetum) } \\
\hline Cornus sanguinea & $\cdot$ & $\cdot$ & . & . & $\cdot$ & . & . & . & 1 & 2 & . & 1 & $\cdot$ & $\cdot$ & $\cdot$ & $\cdot$ & + & + & $\cdot$ & · & + & . & . & $\cdot$ \\
\hline Helleborus occidentalis & . & $\cdot$ & . & . & . & . & . & · & $\cdot$ & + & 1 & + & $\cdot$ & $\cdot$ & $\cdot$ & $\cdot$ & + & $\cdot$ & 1 & + & . & . & . & . \\
\hline Saxifraga hirsuta & . & . & . & . & . & + & . & . & $\cdot$ & . & . & . & $\cdot$ & + & + & . & . & $\cdot$ & . & · & . & 1 & + & · \\
\hline Euonymus europaeus & . & . & · & . & . & . & . & . & . & . & . & . & + & $\cdot$ & $\cdot$ & $\cdot$ & 1 & $\cdot$ & $\cdot$ & . & . & . & . & + \\
\hline Daphne laureola & . & . & . & . & . & . & . & . & $\cdot$ & . & + & + & $\cdot$ & $\cdot$ & . & . & . & $\cdot$ & . & . & . & + & . & $\cdot$ \\
\hline Bromus ramosus & . & . & . & . & . & . & 1 & . & . & . & . & . & . & $\cdot$ & . & 1 & . & . & . & . & . & . & . & · \\
\hline Viola suavis & $\cdot$ & . & . & . & . & . & . & · & $\cdot$ & 1 & . & . & $\cdot$ & $\cdot$ & $\cdot$ & $\cdot$ & . & $\cdot$ & $\cdot$ & $\cdot$ & . & . & . & · \\
\hline
\end{tabular}




\begin{tabular}{|c|c|c|c|c|c|c|c|c|c|c|c|c|c|c|c|c|c|c|c|c|c|c|c|c|}
\hline $\mathrm{N}^{\circ}$ de orden & 1 & $2^{*}$ & $3^{*}$ & 4 & 5 & 6 & 7 & 8 & 9 & $10^{*}$ & $11^{*}$ & $12^{*}$ & 13 & 14 & $15^{*}$ & $16^{*}$ & 17 & 18 & 19 & $20^{*}$ & 21 & 22 & $23^{*}$ & 24 \\
\hline Lilium martagon & . & . & - & $\cdot$ & $\cdot$ & . & $\cdot$ & . & - & . & 1 & - & . & . & . & . & . & 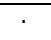 & $\cdot$ & . & . & . & . & . \\
\hline Arbutus unedo & $\cdot$ & $\cdot$ & . & $\cdot$ & . & + & 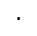 & . & . & . & & . & . & . & . & . & . & & . & & . & . & . & . \\
\hline Equisetum telmateia & . & . & . & . & . & 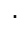 & . & + & 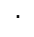 & . & & . & . & . & . & . & . & & . & . & . & . & . & . \\
\hline Saxifraga x polita & . & . & . & . & . & . & . & . & . & . & 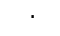 & . & . & + & . & . & . & . & . & 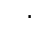 & . & . & . & . \\
\hline Lactuca muralis & . & . & . & . & . & . & . & . & . & . & 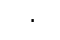 & . & . & . & + & . & . & . & . & 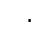 & . & . & . & . \\
\hline Polygonum bistorta & . & . & . & . & . & . & . & . & . & . & & . & . & . & 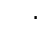 & . & . & + & . & & . & . & . & . \\
\hline
\end{tabular}

Taxones característicos de asociación y unidades superiores

Polystichum setiferum

Brachypodium sylvaticum

Athyrium filix-femina

Primula acaulis

Lonicera hispanica

Dryopteris affinis

Carex remota

Arum italicum

Phyllitis scolopendrium

Ranunculus ficaria

Circaea lutetiana

Luzula sylvatica

Mercurialis perennis

Euphorbia dulcis

Hypericum androsaemum

Oxalis acetosella

Veronica montana

Stachys sylvatica

Solanum dulcamara

Potentilla sterilis

Carex sylvatica

Carex pendula

Symphytum tuberosum

Humulus lupulus

Lysimachia nemorum

Senecio nemorensis

Melica uniflora

Ranunculus tuberosus

Blechnum spicant

Myosotis martini

\begin{tabular}{|c|c|c|c|c|c|c|c|c|c|c|c|c|c|c|c|c|c|c|c|c|c|c|c|}
\hline & & & & & & & & & & & & & & & & & & & & & & & \\
\hline+ & 1 & 1 & 1 & 1 & 1 & . & 2 & 2 & 3 & 1 & 1 & + & 3 & 1 & 1 & 1 & 1 & 3 & 1 & . & 1 & 1 & 1 \\
\hline . & 1 & . & 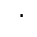 & + & 1 & + & 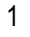 & & 2 & + & 1 & + & 1 & 1 & . & 1 & 2 & + & + & 1 & . & + & 2 \\
\hline 2 & 1 & . & . & 2 & + & 1 & 1 & 1 & 2 & + & 2 & 1 & + & . & + & 2 & 2 & & 4 & 3 & . & 1 & 1 \\
\hline . & 1 & + & + & + & + & . & . & 1 & 1 & 2 & 1 & . & + & + & + & + & + & 1 & ${ }^{\circ}$ & . & . & 1 & + \\
\hline . & 1 & 1 & 1 & + & . & . & . & + & 1 & & 1 & . & 1 & . & . & + & 1 & 1 & 1 & 1 & 1 & + & 1 \\
\hline 1 & 1 & 1 & + & 1 & 1 & . & 1 & 1 & 1 & & 1 & . & 1 & . & . & 1 & 1 & & 1 & . & 1 & & \\
\hline 1 & . & . & . & . & . & . & . & 1 & 1 & + & 1 & . & + & + & . & + & 1 & + & . & + & 1 & 1 & + \\
\hline . & . & + & . & + & . & . & . & 1 & + & + & + & + & 1 & 1 & . & 1 & 1 & + & . & . & . & 1 & 1 \\
\hline . & 1 & 2 & + & . & . & . & . & . & 1 & + & + & . & 2 & 1 & + & + & & 2 & . & . & + & 1 & 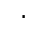 \\
\hline . & . & & 1 & + & & 1 & . & 2 & 1 & 1 & 1 & . & + & 1 & & + & 1 & 1 & & . & . & 1 & 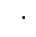 \\
\hline+ & + & 1 & . & . & $\cdot$ & 1 & + & + & 2 & 1 & 1 & + & . & . & + & + & 1 & . & . & . & . & . & . \\
\hline+ & + & . & + & 1 & 1 & . & + & 1 & 1 & . & + & . & . & . & . & . & 1 & . & . & . & 2 & 1 & 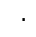 \\
\hline . & + & . & . & + & . & . & . & + & 2 & 1 & + & . & + & + & 1 & + & ${ }^{\prime}$ & 1 & . & . & . & 1 & ${ }^{\prime}$ \\
\hline+ & 1 & ${ }^{\prime}$ & . & + & + & . & . & 1 & 2 & 1 & 1 & . & . & . & 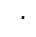 & + & & 1 & & + & . & . & 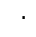 \\
\hline . & . & . & . & + & 1 & . & + & 1 & + & + & + & . & . & . & . & . & + & + & + & . & $\cdot$ & + & 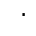 \\
\hline+ & . & . & . & + & 1 & . & + & + & . & . & . & + & 1 & . & . & . & . & 1 & . & . & 1 & 1 & 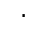 \\
\hline . & 1 & 1 & . & + & $\cdot$ & . & . & + & 1 & + & $\cdot$ & . & + & + & . & . & $\cdot$ & + & $\cdot$ & . & $\cdot$ & 1 & 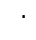 \\
\hline . & . & 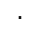 & . & . & . & . & . & + & + & + & 1 & + & & + & 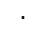 & + & & . & & + & . & + & + \\
\hline . & . & + & . & . & . & + & . & . & . & + & 1 & + & . & . & . & 1 & 1 & . & . & 1 & . & r. & 1 \\
\hline . & + & . & + & . & + & . & . & 1 & . & · & + & . & . & . & . & 1 & + & . & $\cdot$ & . & . & 1 & + \\
\hline+ & + & . & + & . & . & . & + & ${ }^{\circ}$ & . & + & . & . & + & . & + & . & $\cdot$ & . & + & . & . & + & . \\
\hline+ & . & . & . & 3 & $\cdot$ & . & 2 & 1 & . & . & . & $r$ & 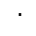 & . & . & . & & 1 & + & . & $\cdot$ & + & . \\
\hline . & + & 1 & . & . & . & . & . & 2 & 1 & 1 & . & + & . & . & . & . & . & + & . & . & . & . & . \\
\hline . & . & + & . & . & . & 1 & . & . & . & . & . & 1 & . & . & . & 1 & 1 & . & . & 1 & . & . & 1 \\
\hline+ & . & . & + & + & . & . & 1 & + & 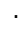 & . & . & + & . & . & . & + & . & . & . & . & . & . & . \\
\hline+ & . & $\cdot$ & . & . & $\cdot$ & . & . & . & . & $\cdot$ & + & . & . & . & . & 1 & 1 & . & & 3 & $\cdot$ & . & + \\
\hline 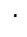 & . & . & . & . & . & . & . & 1 & 1 & + & . & . & 1 & 1 & + & . & & . & & . & $\cdot$ & . & . \\
\hline+ & . & . & . & . & + & . & $\cdot$ & 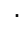 & . & · & . & + & . & . & . & . & $\cdot$ & . & $\cdot$ & . & $\cdot$ & + & . \\
\hline+ & . & . & . & . & . & . & . & + & . & . & . & . & . & . & . & . & + & . & & . & + & 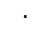 & \\
\hline+ & . & $r$ & . & & & . & 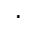 & + & 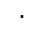 & + & & . & & . & & . & & . & & . & . & & \\
\hline
\end{tabular}

Taxones característicos de Quercetea robori-petraeae y Carpino-Fagetea Euphorbia amygdaloides

Ajuga reptans

Dryopteris filix-mas

Crepis lampsanoides

Stellaria holostea

Polypodium vulgare

Holcus mollis

Poa nemoralis

Teucrium scorodonia

Dryopteris dilatata

Anemone nemorosa

Tamus communis

Aquilegia vulgaris

\begin{tabular}{|c|c|c|c|c|c|c|c|c|c|c|c|c|c|c|c|}
\hline 1 & + & + & + & + & + & & · & + & 1 & & . & 1 & . & 1 & 1 \\
\hline 1 & + & 1 & + & 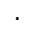 & + & & . & + & + & + & . & & . & + & . \\
\hline . & 2 & . & . & + & . & . & + & 1 & + & . & . & + & . & 1 & . \\
\hline r & 1 & 1 & . & + & + & + & . & . & + & . & . & . & . & + & 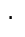 \\
\hline r. & 1 & + & + & . & $\cdot$ & . & 1 & + & + & . & . & + & . & 1 & 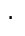 \\
\hline+ & + & . & . & . & 1 & . &. & . & + & . & . & . & . & 1 & . \\
\hline 2 & . & . & . & + & . & . & . & 1 & . & + & . & " & + & . & . \\
\hline & . & . & . & $\cdot$ & + & + & 1 & $\cdot$ & . & ${ }^{\circ}$ & . & & 1 & . & . \\
\hline+ & . & $\cdot$ & . & . & . & 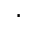 & . & + & + & . & . & • & + & . & . \\
\hline+ & . & $\cdot$ & . & . & $\cdot$ & & · & $\cdot$ & 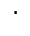 & 1 & - & & 1 & . & \\
\hline+ & + & . & . & . & . & . & $\cdot$ & $\cdot$ & . & r & . & . & · & . & $\cdot$ \\
\hline . & + & + & . & . & $\cdot$ & . & · & $\cdot$ & . & + & . & . & . & . & . \\
\hline$\cdot$ & . & . & . & + & . & . & . & & 1 & & . & & & + & \\
\hline
\end{tabular}

\begin{tabular}{|c|c|c|c|c|c|c|c|c|c|c|c|c|c|c|c|c|c|c|c|c|c|c|c|c|}
\hline \multicolumn{25}{|l|}{ Otros taxones } \\
\hline Rubus sp. & + & + & 1 & 1 & + & + & 1 & 1 & 1 & 1 & 1 & 1 & . & 1 & 1 & 1 & 1 & 1 & 1 & 1 & 1 & 1 & 1 & 1 \\
\hline Geum urbanum & + & 1 & 1 & + & + & + & 1 & + & 2 & 1 & 1 & 1 & 1 & 1 & 1 & + & 1 & 1 & + & & 1 & $\cdot$ & 1 & 1 \\
\hline Geranium robertianum & . & 1 & 1 & 1 & + & . & 1 & 1 & + & 1 & 1 & 1 & + & 1 & 1 & 1 & 1 & + & 1 & 1 & 1 & . & 1 & 1 \\
\hline Heracleum sphondylium & . & + & + & 1 & 2 & + & + & . & 1 & 1 & 1 & + & + & + & + & 1 & + & + & . & . & 1 & . & 1 & 1 \\
\hline Silene dioica & . & 1 & 1 & 1 & 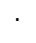 & . & 1 & 2 & 1 & 1 & & 1 & + & 1 & 1 & . & + & 1 & 1 & 3 & 2 & . & 1 & 1 \\
\hline Oenanthe crocata & + & 1 & 1 & . & + & + & & + & + & + & 1 & 2 & + & . & + & . & 1 & + & + & 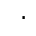 & 1 & . & 1 & 1 \\
\hline Urtica dioica & . & + & 1 & . & . & . & 1 & r & . & + & + & + & + & + & + & + & 1 & + & + & + & . & . & . & 1 \\
\hline Brachypodium rupestre & . & 1 & + & 1 & . & 3 & 1 & . & 1 & + & 1 & . & + & 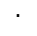 & . & 2 & 1 & 2 & . & . & 1 & . & 1 & 3 \\
\hline Scrophularia auriculata & . & + & + & . & . & 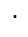 & . & . & + & + & + & + & + & . & . & 1 & 1 & + & . & 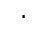 & 1 & . & + & + \\
\hline Alliaria petiolata & . & . & + & + & . & . & + & . & + & + & 1 & + & + & 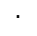 & + & . & + & + & . & & + & . & 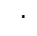 & 1 \\
\hline Dactylis glomerata & . & . & + & 1 & . & . & . & . & 1 & . & & + & + & . & + & + & 1 & 1 & . & . & 1 & . & + & 1 \\
\hline Chrysosplenium oppositifolium & + & 1 & 1 & + & + & . & . & . & . & . & & 1 & . & + & . & . & . & . & 1 & 3 & . & 1 & + & . \\
\hline Ranunculus repens & . & 1 & & 1 & + & . & 1 & . & + & . & & 1 & . & & . & + & + & + & . & & . & 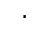 & + & + \\
\hline
\end{tabular}




\begin{tabular}{|c|c|c|c|c|c|c|c|c|c|c|c|c|c|c|c|c|c|c|c|c|c|c|c|c|}
\hline $\mathrm{N}^{0}$ de orden & 1 & $2^{*}$ & $3^{*}$ & 4 & 5 & 6 & 7 & 8 & 9 & $10^{*}$ & $11^{*}$ & $12^{*}$ & 13 & 14 & $15^{*}$ & $16^{*}$ & 17 & 18 & 19 & $20^{*}$ & 21 & 22 & $23^{*}$ & 24 \\
\hline Pentaglottis sempervirens & . & $\cdot$ & . & . & $\cdot$ & . & + & . & + & + & . & + & $\cdot$ & + & + & 1 & . & + & . & $\cdot$ & + & . & + & + \\
\hline Filipendula ulmaria & . & . & . & + & . & . & . & . & $\cdot$ & . & . & 1 & + & 1 & 1 & . & 3 & 3 & . & . & 1 & . & 1 & + \\
\hline Taraxacum gr. officinale & . & + & . & + & + & . & + & . & + & . & . & . & . & $\cdot$ & . & + & $r$ & + & . & . & $r$ & . & + & . \\
\hline Veronica chamaedrys & . & + & + & . & . & . & . & + & . & . & . & . & + & . & . & + & + & 1 & . & . & 1 & . & . & 1 \\
\hline Equisetum arvense & $r$ & . & + & . & . & . & 2 & . & + & . & . & + & . & . & . & + & $\cdot$ & + & . & . & . & . & . & + \\
\hline Clematis vitalba & . & . & $\cdot$ & . & . & . & 1 & . & 1 & 1 & . & + & . & 1 & + & . & . & 1 & . & . & . & . & 1 & . \\
\hline Lapsana communis & . & . & + & . & . & . & + & . & $r$ & . & 1 & + & . & . & . & . & + & . & . & . & + & . & . & + \\
\hline Angelica sylvestris & . & 1 & $\cdot$ & . & . & . & 1 & . & + & 1 & . & . & . & + & + & . & . & . & + & . & . & . & . & . \\
\hline Vicia sepium & . & + & + & + & . & . & . & + & . & . & $\cdot$ & . & . & . & + & 1 & . & . & . & . & . & . & + & . \\
\hline Apium nodiflorum & $r$ & . & . & . & . & . & + & . & . & . & . & 1 & . & . & . & + & + & . & . & . & + & . & . & + \\
\hline Umbilicus rupestris & . & + & . & . & . & . & · & . & + & . & . & . & . & . & . & . & + & + & $r$ & + & . & . & 1 & . \\
\hline Cardamine hirsuta & + & $\cdot$ & + & + & . & . & . & · & + & + & . & . & . & . & . & . & $r$ & $\cdot$ & . & . & $\cdot$ & . & + & . \\
\hline Limniris pseudacorus & . & $\cdot$ & . & . & . & . & 1 & . & $\cdot$ & $\cdot$ & . & 1 & + & . & . & . & + & + & . & . & + & . & . & . \\
\hline Galium aparine & . & . & + & . & . & . & + & . & . & 1 & + & . & . & . & + & + & . & . & . & . & . & . & . & . \\
\hline Lythrum salicaria & . & . & . & . & . & . & . & . & . & . & . & + & . & . & . & . & + & 1 & . & . & 1 & . & . & 1 \\
\hline Calystegia sepium & . & . & . & . & . & . & + & . & . & . & . & 1 & + & . & . & . & 1 & . & . & . & . & . & . & + \\
\hline Asplenium trichomanes & . & . & . & + & . & . & . & . & . & . & . & . & . & + & + & 1 & $\cdot$ & . & . & . & . & . & + & . \\
\hline Prunella vulgaris & . & . & . & . & . & . & . & . & + & . & . & . & . & . & . & + & + & . & . & . & 1 & . & + & . \\
\hline Glechoma hederacea & . & . & $\cdot$ & . & . & . & . & · & . & . & . & + & + & . & . & · & + & 1 & . & . & . & . & . & + \\
\hline Ruscus aculeatus & . & . & . & . & . & + & . & . & + & + & + & . & . & . & . & . & $\cdot$ & . & + & . & . & . & . & . \\
\hline Polygonum hydropiper & . & $\cdot$ & . & . & . & . & . & . & . & . & . & . & . & . & . & . & + & + & . & . & 1 & . & . & 1 \\
\hline Cardamine pratensis & . & + & . & . & . & . & . & . & + & . & . & . & . & . & . & + & $\cdot$ & . & . & 1 & . & . & . & . \\
\hline Cardamine flexuosa & . & $\cdot$ & $\cdot$ & 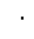 & . & . & . & . & . & . & . & + & + & . & . & . & $\cdot$ & $\cdot$ & . & . & + & . & . & + \\
\hline Sparganium neglectum & . & . & $\cdot$ & $\cdot$ & . & . & . & . & . & . & . & + & . & . & . & . & + & . & . & . & + & . & . & + \\
\hline Pteridum aquilinum & . & $\cdot$ & $\cdot$ & . & . & · & . & $r$ & + & . & . & $\cdot$ & $\cdot$ & $\cdot$ & . & $\cdot$ & + & . & $\cdot$ & . & . & $\cdot$ & . & + \\
\hline
\end{tabular}

Taxones presentes en menos de cuatro inventarios:

$\mathrm{E}_{1}+\mathrm{E}_{2}$ : Frangula alnus: + en 9 y + en 21; llex aquifolium: + en 5 y + en 6; Juglans regia: +en 7; Malus domestica: + en 7; Populus nigra: 1 en 7 y r en 17; Quercus pyrenaica: r en 18; Rosa arvensis: + en 10; Rosa micrantha: + en 7; Rosa villosa: + en 16; Salix caprea: 1 en 16; Salix fragilis: + en 4; Ulmus glabra: 1 en 11. Taxones característicos de asociación y unidades superiores: Elymus caninus: + en 16; Ornithogalum pyrenaicum: + en 11; Pulmonaria longifolia: + en 8 y + en 9; Sanicula europea: + en 23. Taxones característicos de Quercetea robori-petraeae y Carpino-Fagetea: Hyacinthoides non-scripta: + en 9, + en 11 y + en 18; Polystichum aculeatum: + en 22. Otros taxones: Agrostis capillaris: 1 en 1, 1 en 12 y + en 24; Arrhenatherum bulbosum: + en 4, + en 14 y + en 15; Asplenium onopteris: r en 6; Carex lusitánica: + en 12; Carex pilulifera: + en 5 y + en 17; Centaurea rivularis: r en 17; Chelidonium majus: + en 7 y + en 24 ; Cirsium palustre: + en 4 y + en 16; Coincya setigera: r en 9; Conium maculatum: + en 13; Conyza bonariensis: + en 7; Digitalis purpurea: + en 9; Epilobium obscurum: r en 1; Epilobium sp.: + en 7; Festuca gr. nigrescens: + en 16; Fragaria vesca: + en 2, + en 16 y + en 23; Galeopsis tetrahit: + en 7, + en 21 y + en 24; Galium mollugo: r en 8; Galium palustre: + en 1; Geranium lucidum: + en 4, + en 15 y + en 16; Glyceria fluitans: + en 1 y + en 12; Hypericum sp.: 1 en 17; Juncus effusus: + en 12; Juncus heterophyllus: + en 17; Laserpitium duforianum: + en 17; Lycopus europaeus: 1 en 12, + en 17 y + en 21, Meconopsis cambrica: + en 14; Mentha aquatica: + en 12 y + en 17; Mentha suaveolens: + en 17; Oxalis sp.: $r$ en 17; Phalaris arundinacea: 1 en 17,1 en 21 y 1 en 24; Picris hieracioides: + en 9; Poa annua: + en 11; Poa trivialis: + en 16; Polygonatum odoratum: + en 5; Polygonum persicaria: + en 6; Polygonum sp.: + en 7, Polypodium cambricum: + en 15 y + en 19; Pritzelago auerswaldii: + en 16; Rumex obtusifolius: + en 17; Scutellaria minor: + en 17; Silene vulgaris: + en 8; Stellaria media: + en 16; Succisa pratensis: + en 6; Tritonia x crocosmiflora: + en 6 y + en 9; Valeriana montana: + en 9; Veronica anagallis-aquatica: + en 16 .

Procedencia de los inventarios:

1: Lu: A Fonsagrada, Centigosa, Río de Piñeira (646/4774); 2: Lu: As Nogais, Río Valdeparada, entre la villa y Doncos (655/4739); 3: Lu: Baralla, Arroxo, Río de Vilachambre (642/4751); 4: Lu: Samos, Río Lóuzara, aguas abajo del puente de Gundriz (646/4728); 5: Lu: Samos, Río Oribio, aguas abajo de la villa (636/4732); 6: As: Valdés, Río Esba, margen izquierda aguas abajo del puente de la carretera de Brieves a Merás (706/4817); 7: Le: Vega de Valcárce, Ambascasas, Río Valcárce (669/4725); 8: Lu: A Pontenova, Vilarxubín, Fraga de Reigadas, confluencia de los arroyos de Budueiro y Bounote (650/4796); 9: Lu: A Pontenova, Xinzo, Río Eo (646/4798); 10: Lu: As Nogais, As Nogais, Río de Valdeparada (655/4741); 11: Lu: Baralla, Pol, Río Covo (642/4746); 12: Lu: Baralla, Río Neira, aguas arriba del puente entre Rariz y Vilasantán (637/4746); 13: Lu: Baralla, Río Neira, aguas abajo de Baralla (641/4748); 14: Lu: Folgoso do Courel, Río Lor, augas arriba del Puente de Esperante (653/4722); 15: Lu: Folgoso do Courel, Río Lor, entre Ferrería Nova y Ferrería Vella (650/4721); 16: Lu: Folgoso do Courel, Visuña, Río de Visuña, aguas abajo de la confluencia con el Carrozo dos Corvos (658/4718); 17: Lu: Láncara, Río Neira, aguas arriba del puente entre Carracedo y Fuxón (633/4747); 18: Lu: Láncara, Río Neira, entre Bande y Muro (630/4747); 19: Lu: Lonrenzá, Santoadrao, Rego do Castelo, entre As Pantigueiras y A Currega de Abaixo (639/4808); 20: Lu: Mondoñedo, Samordás, Rego de Samordás (629/4805); 21: Lu: O Páramo, Río Sarria, entre Os Pasos y Pobra de S. Xiao (626/4746); 22: Lu: Samos, Río da Portela (646/4727); 23: Lu: Samos, Río Lóuzara, entre Santalla de Arriba y Santalla de Abaixo (642/4724); 24: Lu: Sarria, Río Sarria, bosque de la isla próxima al molino de Oleiros (631/4744). 
Tabla VI. Alisedas cantábricas occidentales (Valeriano pyrenaicae-Alnetum glutinosae) variante hiperoceánica, subvariante típica

\begin{tabular}{|c|c|c|c|c|c|c|c|c|c|c|c|c|c|c|}
\hline$\overline{\mathbf{N}^{\circ} \text { de orden }}$ & 1 & 2 & 3 & 4 & 5 & 6 & 7 & 8 & 9 & 10 & 11 & 12 & 13 & 14 \\
\hline Altitud (m) & 45 & 85 & 85 & 50 & 205 & 45 & 185 & 150 & 95 & 65 & 55 & 60 & 290 & 315 \\
\hline Pendiente $\left({ }^{\circ}\right)$ & $<2$ & 4 & 4 & 6 & 4 & 4 & 8 & 2 & 6 & 4 & $<2$ & 4 & 22 & 4 \\
\hline Orientación & SW & $E$ & NNE & $\mathrm{N}$ & SE & WSW & ENE & $\mathrm{N}$ & $\mathrm{N}$ & NNW & NW & WNW & WNW & $\mathrm{NE}$ \\
\hline Altura de copas (m) & 16 & 18 & 16 & 28 & 26 & 16 & 12 & 14 & 22 & 28 & 12 & 20 & 12 & 22 \\
\hline Cob. $E_{1}(\%)$ & 100 & 100 & 100 & 100 & 85 & 90 & 100 & 80 & 95 & 100 & 90 & 100 & 80 & 95 \\
\hline Cob. $E_{2}(\%)$ & 25 & 25 & 30 & 20 & 15 & 25 & 10 & --- & 30 & 40 & 50 & 30 & 30 & 25 \\
\hline Cob. $E_{3}(\%)$ & 95 & 85 & 80 & 50 & 90 & 65 & 90 & 70 & 85 & 90 & 60 & 90 & 80 & 85 \\
\hline Área $\left(\mathrm{m}^{2}\right)$ & 400 & 200 & 200 & 200 & 400 & 200 & 300 & 120 & 300 & 500 & 300 & 300 & 100 & 500 \\
\hline $\mathrm{N}^{\circ}$ de taxones & 62 & 45 & 48 & 34 & 49 & 44 & 46 & 31 & 51 & 93 & 56 & 77 & 27 & 55 \\
\hline \multicolumn{15}{|c|}{$E_{1}(>4,0 \mathrm{~m})+E_{2}(>1,5-4,0 \mathrm{~m}):$} \\
\hline Alnus glutinosa & 4 & 5 & 4 & 4 & 4 & 3 & 5 & 4 & 5 & 3 & 4 & 4 & 3 & 5 \\
\hline Corylus avellana & 2 & 2 & 1 & 1 & 2 & 3 & 2 & 2 & 1 & 3 & 3 & 2 & 2 & 1 \\
\hline Fraxinus excelsior & 1 & 1 & 2 & 2 & . & + & . & 1 & 1 & 3 & 2 & 3 & . & 1 \\
\hline Laurus nobilis & 2 & 2 & 1 & + & 1 & 1 & 1 & . & 1 & 1 & 1 & 1 & . & . \\
\hline Salix atrocinerea & + & + & 1 & . & 1 & 1 & 1 & . & 1 & 1 & . & . & 1 & + \\
\hline Acer pseudoplatanus & + & 1 & + & 1 & . & . & . & . & 1 & 2 & . & 1 & . & 1 \\
\hline Crataegus monogyna & 1 & 1 & . & 1 & . & . & . & . & + & 1 & 1 & 1 & . & 1 \\
\hline Castanea sativa & 2 & 1 & + & . & 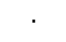 & . & . & 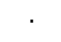 & $\cdot$ & 1 & + & 1 & . & + \\
\hline Quercus robur & 1 & . & + & . & 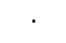 & + & . & . & $\cdot$ & . & + & + & 2 & 1 \\
\hline Sambucus nigra & 1 & 1 & 1 & 1 & + & $\cdot$ & . & . & $\cdot$ & 1 & $\cdot$ & 1 & $\cdot$ & $\cdot$ \\
\hline Frangula alnus & 1 & . & + & . & . & + & . & . & . & + & 1 & + & . & 1 \\
\hline Ulmus glabra & . & . & . & 1 & . & 3 & . & 1 & $\cdot$ & + & 1 & . & . & $\cdot$ \\
\hline Rosa gr. canina & + & + & . & . & . & . & . & . & + & . & . & + & . & + \\
\hline Ligustrum ovalifolium & . & 1 & 2 & . & . & . & . & . & $\cdot$ & . & . & + & . & . \\
\hline Prunus spinosa & + & 1 & . & . & . & . & . & & . & . & . & + & . & . \\
\hline Ilex aquifolium & ${ }^{\circ}$ & & . & . & 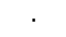 & . & . & & . & 1 & + & + & . & . \\
\hline
\end{tabular}

\section{$E_{3}(<1,5 \mathrm{~m}):$}

Taxones diferenciales frente a Senecioni-Alnetum

Valeriana pyrenaica

Chaerophyllum hirsutum

Festuca gigantea

Dryopteris aemula

Scrophularia alpestris

Adenostyles alpina

Avenella flexuosa

Narcissus pseudonarcissus s.l.

Quercus petraea $\times$ Q. robur

Smilax aspera

Cardamine raphanifolia

Saxifraga lepismigena

Erica mackaiana

Lastrea limbosperma

\begin{tabular}{|c|c|c|c|c|c|c|c|c|c|c|c|c|c|c|}
\hline \multicolumn{15}{|c|}{ Taxones diferenciales frente a Hyperico-Alnetum } \\
\hline Hedera hibernica & 2 & 3 & $\cdot$ & 2 & 2 & 2 & 2 & + & 2 & 2 & 1 & 2 & 1 & 3 \\
\hline Osmunda regalis & 1 & . & 1 & . & 1 & + & 1 & 1 & 2 & 1 & 2 & 3 & 1 & 1 \\
\hline Saxifraga spathularis & . & . & . & . & 1 & + & 1 & + & 1 & + & 1 & 1 & 1 & 2 \\
\hline Viola palustris & . & . & + & . & + & 1 & . & + & 1 & . & 1 & 1 & . & 1 \\
\hline Omphalodes nitida & + & . & . & . & + & + & + & . & + & + & + & + & . & . \\
\hline Carex reuteriana & $\cdot$ & . & . & . & 1 & 1 & . & . & 1 & + & 1 & 1 & . & 1 \\
\hline Deschampsia subtriflora & . & . & . & . & + & + & . & . & . & + & + & + & . & 2 \\
\hline Pyrus cordata & . & . & . & . & + & . & . & . & . & + & 1 & . & 1 & + \\
\hline Solidago virgaurea & . & . & + & . & + & + & . & . & . & . & + & . & . & + \\
\hline Betula pubescens & $\cdot$ & . & . & . & . & . & . & . & . & 1 & . & . & 1 & + \\
\hline Erica arborea & . & . & . & . & . & . & . & . & . & . & $\mathrm{r}$ & . & 1 & + \\
\hline Arbutus unedo & . & . & . & . & . & . & . & . & . & . & 1 & . & 1 & 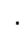 \\
\hline Hieracium umbellatum & . & . & . & . & . & . & . & . & . & + & 1 & . & . & . \\
\hline Conopodium majus & . & . & . & . & . & . & . & . & . & 1 & . & + & . & . \\
\hline Vaccinium myrtillus & . & . & . & . & . & . & . & . & . & . & + & . & + & . \\
\hline Euphorbia hyberna & . & . & . & . & . & . & + & . & . & & . & . & 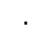 & . \\
\hline Narcissus asturiensis & . & . & . & . & . & . & + & . & . & & . & . & . & . \\
\hline
\end{tabular}




\begin{tabular}{|c|c|c|c|c|c|c|c|c|c|c|c|c|c|c|}
\hline $\mathrm{N}^{\circ}$ de orden & 1 & 2 & 3 & 4 & 5 & 6 & 7 & 8 & 9 & 10 & 11 & 12 & 13 & 14 \\
\hline Caltha palustris & · & ${ }^{\circ}$ & & . & . & '. & · & $\cdot$ & & + & $\cdot$ & & . & $\cdot$ \\
\hline Narcissus triandrus & . & 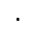 & . & . & ${ }^{\prime}$ & . & . & . & & + & 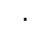 & . & $\cdot$ & $\cdot$ \\
\hline Physospermum cornubiense & 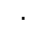 & . & ${ }^{\circ}$ & . & ${ }^{\circ}$ & r. & . & $\cdot$ & & $\cdot$ & + & . & . & . \\
\hline Allium victorialis & . & . & . & . & . & . & . & . & & . & . & + & . & . \\
\hline
\end{tabular}

Taxones diferenciales de variante subhiperoceánica

\begin{tabular}{|c|c|c|c|c|c|c|c|c|c|c|c|c|c|c|}
\hline Woodwardia radicans & + & 1 & 1 & 1 & + & $\cdot$ & 2 & 4 & 1 & 1 & 1 & + & . & \\
\hline Hymenophyllum tunbrigense & . & . & & ${ }^{\circ}$ & ${ }^{\prime}$ & + & . & . & 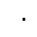 & . & 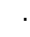 & & 1 & + \\
\hline Davallia canariensis & . & . & . & . & . & + & . & + & $\cdot$ & . & + & 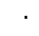 & . & . \\
\hline Culcita macrocarpa & . & . & . & . & . & . & . & . & . & . & . & . & 3 & ${ }^{\circ}$ \\
\hline Stenogramma pozoi & . & . & . & + & . & . & . & . & . & . & . & . & . & \\
\hline
\end{tabular}

Taxones característicos de asociación y unidades superiores

\begin{tabular}{|c|c|c|c|c|c|c|c|c|c|c|c|c|c|c|}
\hline Athyrium filix-femina & 2 & 2 & 3 & 1 & 2 & 2 & 3 & 2 & 1 & 2 & 1 & 2 & $\cdot$ & 3 \\
\hline Hypericum androsaemum & 1 & + & + & + & + & . & + & + & + & + & + & + & · & + \\
\hline Viola riviniana & 1 & 1 & 1 & $\cdot$ & 1 & 1 & 1 & · & 1 & 1 & 1 & 1 & · & 1 \\
\hline Euphorbia dulcis & + & · & + & · & + & 1 & 3 & · & 2 & 1 & 1 & 2 & - & 1 \\
\hline Lonicera hispanica & 2 & · & - & $\cdot$ & 1 & 1 & 1 & + & 1 & + & 1 & $\cdot$ & 2 & 1 \\
\hline Primula acaulis & + & + & . & . & . & + & 1 & $r$ & 1 & 1 & . & 1 & . & . \\
\hline Polystichum setiferum & $\cdot$ & 1 & · & 3 & . & 1 & 1 & 1 & $\cdot$ & + & $\cdot$ & 1 & - & . \\
\hline Mercurialis perennis & + & + & . & + & . & . & + & . & $\cdot$ & 1 & · & 2 & . & + \\
\hline Brachypodium sylvaticum & 1 & 1 & + & $\cdot$ & · & · & 1 & · & + & 1 & $\cdot$ & 1 & $\cdot$ & · \\
\hline Carex pendula & 1 & 2 & 1 & 1 & · & · & $\cdot$ & $\cdot$ & $\cdot$ & + & $\cdot$ & 1 & · & · \\
\hline Pulmonaria longifolia & + & · & · & $\cdot$ & + & · & + & - & 1 & 1 & $\cdot$ & 1 & $\cdot$ & · \\
\hline Ranunculus ficaria & + & · & · & · & + & . & . & . & 1 & 1 & . & 1 & . & . \\
\hline Veronica montana & + & · & - & + & $\cdot$ & · & · & . & + & 1 & $\cdot$ & 1 & $\cdot$ & $\cdot$ \\
\hline Ranunculus tuberosus & $\cdot$ & · & · & · & - & + & · & $\cdot$ & + & 1 & + & 1 & $\cdot$ & . \\
\hline
\end{tabular}

Ranunculus tuberosus

Taxones característicos de Quercetea robori-petraeae y Carpino-Fagetea

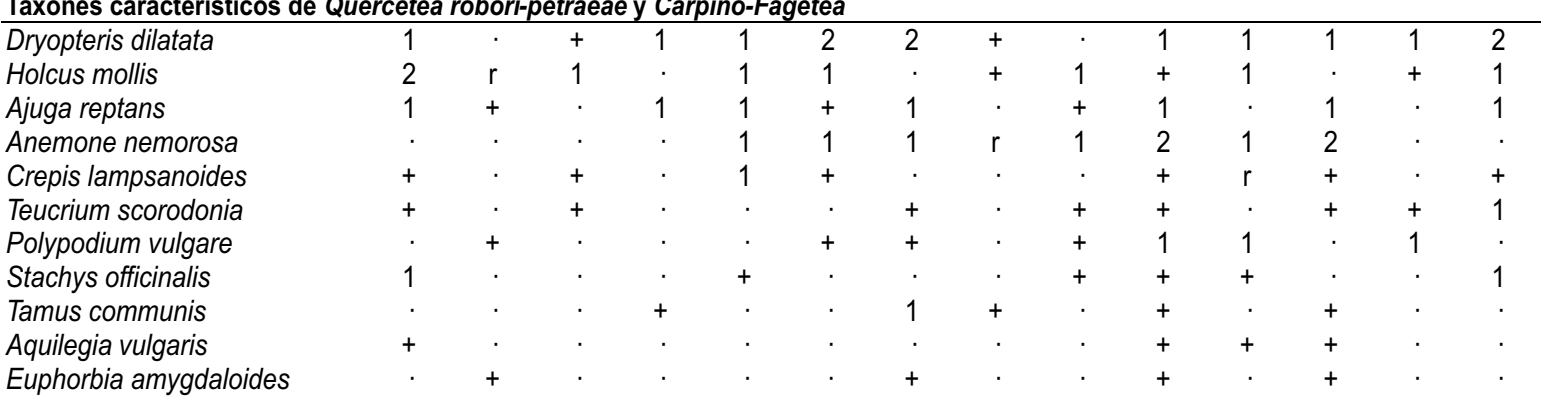

\section{Otros taxones}

\section{Rubus sp.}

Ruscus aculeatus

Oenanthe crocata

Angelica sylvestris

Geranium robertianum

Dactylis glomerata

Silene dioica

Brachypodium rupestre

Cardamine pratensis

Heracleum sphondylium

Rumex acetosa

Pteridum aquilinum

Geum urbanum

Scrophularia auriculata

Ranunculus repens

Centaurea rivularis

Prunella vulgaris

Umbilicus rupestris

\begin{tabular}{|c|c|c|c|c|c|c|c|c|c|c|c|c|c|}
\hline+ & + & 1 & 1 & 1 & + & + & 1 &. & + & 1 & 1 & 1 & 1 \\
\hline 1 & + & + & + & + & + & $\cdot$ & . & + & + & $\cdot$ & + & $\cdot$ & + \\
\hline 2 & . & + & 1 & . & . & + & . & 1 & 1 & 1 & + & $\cdot$ & . \\
\hline . & 1 & 1 & 1 & + & . & $\cdot$ & . & + & 1 & . & + & $\cdot$ & . \\
\hline . & 1 & . & 1 & + & . & + & $r$ & . & + & . & + & $\cdot$ & . \\
\hline+ & . & + & . & 1 & . & . & $r$ & . & + & $r$ & . & $\cdot$ & 1 \\
\hline 1 & 2 & 1 & 1 & 1 & . & . & . & . & . & . & + & . & . \\
\hline . & . & $\cdot$ & . & . & . & + & . & + & + & 1 & + & $\cdot$ & 2 \\
\hline$\cdot$ & 1 & $\cdot$ & 1 & $\cdot$ & 1 & + & . & $\cdot$ & + & . & $\cdot$ & $\cdot$ & . \\
\hline$\cdot$ & 1 & $\cdot$ & . & . & $\cdot$ & 1 & . & + & + & . & + & $\cdot$ & . \\
\hline . & . & + & . & . & . & . & . & . & + & + & + & + & . \\
\hline+ & . & $r$ & . & . & . & . & . & . & $\cdot$ & + & + & $\cdot$ & + \\
\hline+ & 1 & $\cdot$ & . & $\cdot$ & $\cdot$ & $\cdot$ & . & . & + & . & + & $\cdot$ & . \\
\hline 1 & . & + & + & . & . & + & . & . & . & . & . & . & . \\
\hline$\cdot$ & . & + & . & + & . & $\cdot$ & . & $\cdot$ & + & . & . & $\cdot$ & + \\
\hline . & . & $\cdot$ & . & + & . & $\cdot$ & . & + & $\cdot$ & + & . & $\cdot$ & + \\
\hline$\cdot$ & . & $\cdot$ & . & $\cdot$ & $\cdot$ & $\cdot$ & $r$ & . & + & . & + & $\cdot$ & + \\
\hline . & . & $\cdot$ & . & $\cdot$ & 1 & $\cdot$ & . & . & + & . & . & 1 & . \\
\hline . & . & . & . & + & + & $\cdot$ & + & $\cdot$ & $\cdot$ & . & . & $\cdot$ & . \\
\hline
\end{tabular}


Taxones presentes en menos de cuatro inventarios:

$\mathrm{E}_{1}+\mathrm{E}_{2}$ : Acacia melanoxylon: + en 1; Buxus sempervirens: + en 10; Cytisus scoparius: $r$ en 11 y $r$ en 14; Prunus avium: $r$ en 12; Prunus laurocerasus: 1 en 10; Rosa micrantha: + en 10. Taxones característicos de asociación y unidades superiores: Arum italicum: + en 6 y + en 10; Carex sylvatica: + en 9, + en 10 y + en 12; Circaea lutetiana: + en 2, + en 10 y + en 12; Melica uniflora: + en 10; Phyllitis scolopendrium: + en 4 y 1 en 8; Potentilla montana: + en 7; Potentilla sterilis: + en 3 y + en 12; Sanicula europea: + en 9,1 en 10 y 2 en 12; Stachys sylvatica: 3 en 2; Symphytum tuberosum: 1 en 1 y + en 12. Taxones característicos de Quercetea robori-petraeae y Carpino-Fagetea: Dryopteris filix-mas: 1 en 8; Hyacinthoides non-scripta: + en 10 y 1 en 12; Lathyrus linifolius: r en 9, + en 10 y + en 11; Luzula forsteri: + en 10; Melampyrum pratense: + en 10; Polygonatum verticillatum: + en 7; Stellaria holostea: + en 7 y + en 10. Otros taxones: Agrostis capillaris: + en 14; Anthoxanthum amarum: 2 en 5; Anthoxanthum odoratum: + en 10; Asphodelus lusitanicus: 1 en 1 y + en 10; Cardamine flexuosa: + en 1; Cardamine hirsuta: + en 4 y + en 6; Carex cariophyllea: + en 12; Carex cf. umbrosa: + en 10; Carex pilulifera: + en 9; Carum verticillatum: + en 11; Cirsium palustre: $r$ en 3; Cruciata glabra: + en 14; Daboecia cantabrica: + en 14; Digitalis purpurea: + en 1 y r en 6; Equisetum arvense: + en 12; Fragaria vesca: + en 12; Glechoma hederácea: + en 3; Lamium maculatum: 1 en 5; Lapsana communis: 1 en 9; Lythrum salicaria: 1 en 11; Mentha aquatica: + en 2 y + en 12; Molinia caerulea: 1 en 11; Narcissus bulbocodium: + en 10; Peucedanum gallicum: + en 11; Picris hieracioides: r en 1 y + en 3; Polygonatum odoratum: + en 7; Polygonum persicaria: + en 3; Selinum broteri: + en 10; Sibthorpia europea: + en 14; Succisa pratensis: + en 5 y + en 14.

\section{Procedencia de los inventarios:}

1: As: Castropol, Piñera, Río de Fornelo, augas arriba do Caserío Berruga (662/4820); 2: As: Cudillero, Río Ferrera, aguas abajo del puente de la carretera a Foyedo (727/4825); 3: As: Cudillero, Río Uncín, enfrente a El Gallinero (724/4823); 4: As: Valdés, Río de Ferrera, aguas arriba de su unión con el Río Esba (706/4817); 5: As: Valdés, Río Negro, entre Belén e Buseco (693/4815); 6: C: A Capela, P.Nat. Fragas do Eume, Río de Sesín, aguas abajo del Monasterio de Caaveiro (575/4807); 7: C: Cariño, A Pedra, O Castro, margen derecha del Rego Lourido (588/4842); 8: C: Cariño, O Seixo, Río Quiza (586/4838); 9: C: Cerdido, Fraga dos Casás, Río Mera (588/4826); 10: C: Cerdido, Os Casás, entre A Casanova y O Covelo, margen izquierda del Río Mera (588/4828); 11: C: Monfero, P.Nat. Fragas do Eume, Río Eume, margen izquierda entre el Rego da Vaca y el Rego do Rodeiro (576/4806); 12: C: Ortigueira, O Ermo, A Fraga, Rego da Pulgueira, aguas arriba de Ponte da Fraga (589/4828); 13: C: San Sadurniño, Igrexafeita, Rego de Mariaqueira (579/4815); 14: Lu: Alfoz, Río Ouro, aguas arriba del puente de la carretera de O Cadramón a O Pereiro (621/4815). 
Tabla VII. Alisedas cantábricas occidentales (Valeriano pyrenaicae-Alnetum glutinosae): 1-6: variante hiperoceánica, subvariante mesotrofa; 7-13: variante suboceánica,variante típica; 14-25: variante suboceánica, subvariante mesotrofa ${ }^{*}$ presencia de rocas carbonatadas en el tramo de cauce estudiado)

\begin{tabular}{|c|c|c|c|c|c|c|c|c|c|c|c|c|c|c|c|c|c|c|c|c|c|c|c|c|c|}
\hline $\begin{array}{l}\mathrm{N}^{0} \text { de orden } \\
\text { Altitud }(\mathrm{m})\end{array}$ & $\begin{array}{c}1 \\
75\end{array}$ & $\begin{array}{c}2^{*} \\
365\end{array}$ & $\begin{array}{c}3^{*} \\
215\end{array}$ & $\begin{array}{c}4^{*} \\
260\end{array}$ & $\begin{array}{l}5 \\
20\end{array}$ & \begin{tabular}{c|c}
6 & -1 \\
145 & \\
\end{tabular} & $\begin{array}{l}7 \\
7\end{array}$ & $\begin{array}{l}8 \\
3\end{array}$ & $\begin{array}{c}9 \\
230\end{array}$ & $\begin{array}{l}10 \\
10\end{array}$ & $\begin{array}{c}11 \\
290\end{array}$ & $\begin{array}{c}12 \\
275\end{array}$ & \begin{tabular}{c|}
13 \\
225
\end{tabular} & $\begin{array}{c}14 \\
340\end{array}$ & $\begin{array}{c}15 \\
305\end{array}$ & $\begin{array}{c}16 \\
875\end{array}$ & $\begin{array}{c}17 \\
275\end{array}$ & $\begin{array}{c}18 \\
270\end{array}$ & $\begin{array}{c}19 \\
315\end{array}$ & $\begin{array}{c}20 \\
383\end{array}$ & $\begin{array}{c}21 \\
595\end{array}$ & $\begin{array}{c}22 \\
415\end{array}$ & $\begin{array}{c}23 \\
285\end{array}$ & $\begin{array}{c}24 \\
290\end{array}$ & $\begin{array}{r}25 \\
295\end{array}$ \\
\hline Pendiente $\left(^{\circ}\right)$ & 4 & 4 & 2 & 10 & $<2$ & 6 & 2 & 4 & 4 & $<2$ & $<2$ & $<2$ & $<2$ & 2 & $<2$ & 2 & $<2$ & $<2$ & $<2$ & 4 & 6 & 2 & 2 & 2 & $<2$ \\
\hline Orientación & ENE & $E$ & W & NNW & $\mathrm{E}$ & NW & NNW & $E$ & SW & W & $E$ & NW & I SSE & NNW & W & SW & $S$ & SSW & SSW & SW & SW & SSW & SSW & $S$ & E \\
\hline Altura de copas (m) & 20 & 18 & 16 & 18 & 14 & 10 & $10-18$ & $16-30$ & 18 & 18 & 20 & 18 & 20 & 20 & 20 & 20 & 26 & 24 & 30 & 20 & 24 & 20 & 28 & 20 & 24 \\
\hline Cob. $E_{1}(\%)$ & 100 & 100 & 90 & 90 & 100 & 90 & 90 & 100 & 100 & 100 & 100 & 100 & 90 & 90 & 95 & 100 & 100 & 90 & 100 & 100 & 90 & 100 & 100 & 100 & 100 \\
\hline Cob. $E_{2}(\%)$ & 15 & 15 & 30 & 20 & 40 & 20 & 40 & 40 & 15 & 52 & 25 & 30 & 35 & 15 & 35 & 55 & 25 & 30 & 20 & 35 & 35 & 35 & 40 & 35 & 20 \\
\hline Cob. $\mathrm{E}_{3}(\%)$ & 80 & 75 & 70 & 85 & 75 & 90 & 90 & 70 & 70 & 90 & 100 & 95 & 80 & 90 & 85 & 90 & 90 & 90 & 85 & 85 & 70 & 85 & 80 & 90 & 90 \\
\hline Área $\left(\mathrm{m}^{2}\right)$ & 200 & 300 & 300 & 300 & 250 & 400 & 500 & 600 & 500 & 300 & 500 & 500 & 300 & 500 & 500 & 500 & 500 & 300 & 600 & 500 & 300 & 400 & 400 & 300 & 400 \\
\hline $\mathrm{N}^{0}$ de taxones & 50 & 47 & 53 & 39 & 63 & $56 !$ & 96 & 90 & 79 & 47 & 52 & 59 & 72 & 111 & 101 & 76 & 55 & 67 & 92 & 54 & 56 & 51 & 54 & 57 & 64 \\
\hline \multicolumn{26}{|l|}{$E_{1}(>4,0 m)+E_{2}(>1,5-4,0 \mathrm{~m}):$} \\
\hline Alnus glutinosa & 4 & 4 & 3 & 4 & 4 & 3 & 3 & 3 & 5 & 4 & 4 & 4 & 4 & 4 & 3 & 4 & 4 & 4 & 4 & 3 & 5 & 4 & 4 & 5 & 5 \\
\hline Salix atrocinerea & 1 & 1 & 2 & . & 1 & 3 & 1 & 1 & 1 & 2 & 2 & 1 & 1 & 2 & 1 & 1 & 2 & 1 & . & 1 & + & 1 & 1 & 1 & 1 \\
\hline Fraxinus excelsior & 2 & . & 3 & 2 & 1 & 1 & 1 & 2 & + & 1 & . & . & + & 1 & 1 & 3 & . & 1 & 2 & 3 & 1 & 3 & 2 & 1 & 2 \\
\hline Corylus avellana & 1 & 2 & 1 & 1 & 3 & 1 & 1 & 2 & 1 & 1 & . & . & 2 & 1 & + & 1 & + & + & 1 & 1 & 2 & + & 2 & & \\
\hline Acer pseudoplatanus & 1 & 1 & 2 & 1 & . & . & 1 & 2 & 1 & . & . & . & 2 & $\mathrm{r}$ & 2 & 1 & + & 3 & 2 & 3 & 1 & . & 2 & 1 & 2 \\
\hline Crataegus monogyna & & 1 & . & . & . & . & 1 & 1 & 1 & . & 1 & 1 & 1 & 1 & 1 & . & . & 1 & + & 1 & 1 & + & 1 & 1 & 1 \\
\hline Quercus robur & 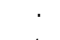 & 1 & . & . & . & . & 1 & + & . & + & 1 & 1 & 1 & 2 & 1 & + & 2 & 1 & + & + & 1 & 1 & . & . & $\cdot$ \\
\hline Sambucus nigra & 1 & . & 1 & 1 & . & 1 & . & . & 1 & + & . & & & + & + & . & . & 1 & + & 1 & . & 1 & + & + & 1 \\
\hline Rosa gr. canina & . & . & + & . & . & . & . & . & + & $\cdot$ & . & 1 & + & 2 & + & 1 & 1 & + & + & + & . & + & 1 & . & + \\
\hline Laurus nobilis & 1 & . & 2 & 2 & 2 & 1 & 1 & 2 & + & 2 & . & 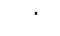 & & & & . & . & . & + & 1 & . & 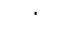 & 2 & 1 & \\
\hline Prunus spinosa & 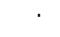 & . & + & . & . & . & 1 & + & + & . & + & . & 1 & + & 1 & . & . & + & + & . & . & + & 1 & . & 1 \\
\hline Frangula alnus & & . & . & . & . & . & 1 & 1 & + & 1 & 1 & + & & + & 2 & . & 1 & & . & & . & . & . & . & + \\
\hline Castanea sativa & . & . & 1 & . & . & 1 & 1 & + & 1 & . & . & . & & . & $r$ & . & . & + & . & . & + & 1 & . & . & . \\
\hline Prunus avium & & . & . & . & $\cdot$ & . & . & . & 1 & . & 1 & 1 & & . & + & . & . & 1 & . & . & . & + & + & + & $r$ \\
\hline Juglans regia & . & . & . & . & . & . & . & . & + & . & . & . & & . & + & $r$ & . & . & . & + & . & . & $r$ & . & $\mathrm{r}$ \\
\hline Ulmus glabra & & . & . & i & . & . & 2 & 2 & . & $\cdot$ & . & . & . & . & 1 & + & . & . & . & . & . & . & . & . & \\
\hline Ilex aquifolium & & 2 & . & 1 & . & . & + & . & . & 1 & . & . & . & . & . & . & . & . & . & . & . & . & . & . & \\
\hline Populus nigra & & . & . & . & . & . & . & . & . & 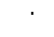 & . & 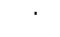 & + & 2 & & 1 & . & . & . & 1 & . & 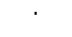 & . & - & \\
\hline \multicolumn{26}{|c|}{$\begin{array}{l}E_{3}(<1,5 \mathrm{~m}): \\
\text { Taxones diferenciales frente a Hyperico-Alnetum }\end{array}$} \\
\hline Hedera hibernica & 2 & 1 & 2 & 1 & 2 & 2 & 2 & 2 & 1 & 2 & 2 & 2 & 1 & 3 & 2 & 2 & 2 & 2 & 2 & 3 & 1 & 2 & 3 & 2 & 2 \\
\hline Carex reuteriana & + & . & . & . & . & . & 1 & 1 & 2 & 1 & . & 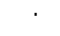 & 2 & 2 & 1 & 1 & + & . & 1 & + & 1 & 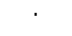 & 1 & + & 2 \\
\hline Osmunda regalis & 2 & $r$ & + & . & 1 & . & 2 & 1 & 1 & 2 & . & + & 1 & + & & . & + & . & + & . & . & + & + & . & + \\
\hline Omphalodes nitida & . & . & . & . & + & . & + & 1 & . & . & + & 1 & + & + & + & . & + & . & . & . & + & + & . & . & . \\
\hline Saxifraga spathularis & . & 1 & . & . & 1 & . & 1 & + & . & . & . & . & & . & . & . & . & . & . & 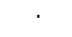 & 1 & . & . & 1 & \\
\hline Luzula forsteri & . & . & . & . & . & . & . & . & + & . & . & . & + & . & 1 & . & + & . & + & . & . & . & . & . & . \\
\hline Hieracium umbellatum & . & . & . & . & . & . & + & . & . & . & . & . & + & + & 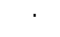 & . & . & . & & . & . & . & + & + & \\
\hline Deschampsia subtriflora & . & . & + & . & + & . & + & + & . & . & . & . & & . & . & . & . & . & . & 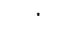 & . & . & . & . & . \\
\hline Quercus pyrenaica & & . & . & . & . & . & . & . & . & . & 2 & . & & & + & $r$ & . & . & . & . & . & . & . & $r$ & . \\
\hline Pyrus cordata & . & $\cdot$ & $\cdot$ & . & $\cdot$ & 1 & 1 & . & . & . & . & . & & . & $r$ & . & . & . & . & . & . & . & . & . & \\
\hline Physospermum cornubiense & & . & . & . & + & . & + & 1 & . & . & . & . & & . & . & . & . & . & . & & . & . & . & . & \\
\hline Solidago virgaurea & . & . & . & . & + & . & . & + & . & . & . & . & & . & . & . & . & . & . & . & . & . & . & + & \\
\hline Aquilegia dichroa & & . & . & . & $\cdot$ & . & + & + & . & . & . & . & & . & . & . & . & . & + & & . & . & . & . & \\
\hline Carex laevigata & & . & . & . & . & . & + & . & . & . & + & . & & + & . & . & . & . & . & . & . & . & . & . & \\
\hline Erica arborea & & r & . & . & . & . & + & . & . & 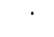 & . & & + & & . & . & . & . & . & & . & . & 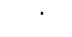 & . & \\
\hline Betula pubescens & & . & . & . & . & . & 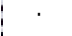 & 2 & . & . & . & . & & & . & . & . & . & . & & 1 & . & . & . & \\
\hline Viola palustris & . & $\cdot$ & . & . & $\cdot$ & . & 1 & 1 & . & $\cdot$ & . & . & & . & . & . & . & . & . & . & · & - & . & - & \\
\hline Narcissus asturiensis & . & . & . & . & . & . & + & . & . & . & . & . & & 1 & . & . & . & . & . & . & . & . & . & . & . \\
\hline Arbutus unedo & & . & . & . & . & . & + & . & . & . & . & . & & . & . & . & . & . & . & & + & . & . & . & \\
\hline Narcissus triandrus & & . & . & . & . & . & + & + & . & $\cdot$ & . & . & & . & . & . & . & . & . & . & . & . & . & . & \\
\hline Allium victorialis & & . & . & . & . & . & + & . & . & . & . & . & & . & . & . & . & . & . & . & . & . & . & . & \\
\hline Conopodium majus & & . & . & . & . & . & + & . & . & . & . & . & & . & . & . & . & . & . & . & . & . & . & . & . \\
\hline Peucedanum lancifolium & & $\cdot$ & . & . & . & . & . & + & . & . & . & . & & 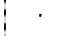 & & . & . & . & . & & . & . & . & . & \\
\hline Angelica major & & $\cdot$ & . & . & . & . & . & . & 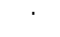 & 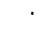 & . & & & + & & . & . & . & . & & . & . & . & 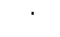 & \\
\hline Euphorbia hyberna & & . & . & . & . & . & . & . & . & . & . & . & & . & . & + & . & . & . & & . & . & . & 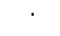 & \\
\hline Caltha palustris & & . & . & . & . & . & . & + & . & 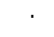 & . & 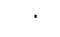 & & & & . & . & . & . & & . & . & . & . & \\
\hline Ceratocapnos claviculata & & . & . & . & 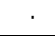 & . & . & . & . & . & . & r & & . & . & . & . & . & . & . & . & . & . & . & \\
\hline
\end{tabular}




\begin{tabular}{|c|c|c|c|c|c|c|c|c|c|c|c|c|c|c|c|c|c|c|c|c|c|c|c|c|c|}
\hline $\mathrm{N}^{0}$ de orden & 1 & $2^{*}$ & $3^{*}$ & $4^{*}$ & 5 & $6 !$ & 7 & 8 & 9 & 10 & 11 & 12 & 13 & 14 & 15 & 16 & 17 & 18 & 19 & 20 & 21 & 22 & 23 & 24 & 25 \\
\hline \multicolumn{26}{|c|}{ Taxones diferenciales frente Senecioni -Alnetum } \\
\hline Festuca gigantea & 1 & + & + & + & + & . & + & + & 1 & . & . & . & 2 & 1 & 1 & + & & . & $\cdot$ & . & $\cdot$ & . & . & . & 1 \\
\hline Chaerophyllum hirsutum & 1 & 1 & + & 3 & + & 1 & & . & . & + & . & . & . & + & + & 1 & & . & + & . & + & . & 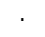 & . & . \\
\hline Valeriana pyrenaica & 1 & + & 1 & 1 & 1 & 2 & & . & . & . & . & . & . & . & . & 1 & & . & . & . & . & . & . & + & + \\
\hline Chamaeiris foetidissima & . & . & r. & . & . & 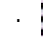 & & . & . & . & + & . & . & . & 1 & . & + & + & + & + & . & . & . & . & + \\
\hline Helleborus foetidus & . & . & . & . & & + & . & . & . & . & . & . & . & . & . & . & + & . & . & . & . & . & . & + & . \\
\hline Scrophularia alpestris & . & + & . & 1 & . & . & ${ }^{\prime}$ & . & . & . & . & . & . & . & . & . & & . & . & . & . & . & . & . & . \\
\hline Cardamine raphanifolia & . & $\cdot$ & 1 & . & . & + & & . & . & . & . & . & . & . & . & . & & . & . & . & . & . & . & . & . \\
\hline Adenostyles alpina & . & . & 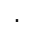 & . & + & . & . & . & . & . & . & . & . & . & . & 1 & & . & . & . & . & . & . & . & . \\
\hline Dryopteris aemula & . & . & . & . & . & + & . & . & . & . & . & . & . & . & . & . & & . & . & . & . & . & . & . & . \\
\hline Erica mackaiana & . & . & . & . & . & . & + & . & . & . & . & . & . & . & . & . & & . & . & . & . & . & . & . & . \\
\hline Smilax aspera & . & . & . & . & . & . & + & . & . & . & . & . & . & . & . & . & & . & . & . & . & . & . & . & . \\
\hline Narcissus pseudonarcissus s.l. & . & . & . & . & . & . & . & . & . & . & . & . & . & . & . & . & & . & . & . & . & . & . & . & + \\
\hline Pimpinella major & . & . & . & . & . & . & & . & $\mathrm{r}$ & . & . & . & . & . & . & . & & . & . & . & . & . & . & . & . \\
\hline Quercus petraea & . & . & & & . & $\cdot$ & & . & . & . & . & & . & . & . & $r$ & & . & & . & . & . & . & . & . \\
\hline
\end{tabular}

Taxones diferenciales de variante subhiperoceánica

\section{Woodwardia radicans}

Hymenophyllum tunbrigense

Vandenboschia speciosa

\begin{tabular}{|c|c|c|c|c|c|c|c|c|c|c|c|c|c|c|c|c|c|c|c|c|c|c|c|c|c|}
\hline \multicolumn{26}{|c|}{ Taxones diferenciales de variantes semihiperoceánicas } \\
\hline Fraxinus angustifolia & . & & - & & . & & 2 & 3 & 1 & $\cdot$ & 1 & 1 & 3 & + & 1 & & 3 & 1 & & . & . & 2 & . & 1 & 1 \\
\hline Ulmus minor & . & & . & & . & & . & . & . & . & & 2 & & 2 & 2 & & 2 & + & 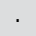 & + & 1 & . & . & . & + \\
\hline Faxinus excelsior $x$ F. angustifolia & . & & . & & . & & 1 & . & 2 & . & & 1 & & 2 & 1 & & & 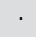 & 1 & . & . & . & 1 & . & 1 \\
\hline Salix salviifolia & . & & . & & . & & . & . & . & 1 & . & . & . & . & . & 1 & 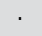 & . & . & . & . & . & . & + & . \\
\hline Salix atrocinerea x S. salviifolia & $\cdot$ & . & $\cdot$ & & · & · & $\cdot$ & $\cdot$ & $\cdot$ & $\cdot$ & $\cdot$ & $\cdot$ & $\cdot$ & . & $\cdot$ & $\cdot$ & & . & + & . & . & . & . & . & . \\
\hline \multicolumn{26}{|c|}{ Taxones diferenciales de subvariantes mesotrofas (y dif. frente a Senecioni-Alnetum) } \\
\hline Saxifraga hirsuta & 1 & + & 1 & & . & . & $\cdot$ & . & . & $\cdot$ & . & . & . & . & . & $\cdot$ & $\cdot$ & . & $\cdot$ & $\cdot$ & + & $\cdot$ & . & . & . \\
\hline Helleborus occidentalis & . & & + & + & . & + & . & . & . & . & 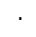 & . & . & . & . & . & & . & . & . & . & . & . & · & . \\
\hline Equisetum telmateia & . & . & . & 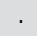 & 2 & 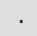 & . & . & . & . & . & . & . & . & . & . & . & . & . & . & . & . & . & . & . \\
\hline Saxifraga x polita & . & + & . & . & . & . & . & . & . & . & . & . & . & . & . & . & . & . & . & . & . & . & . & . & . \\
\hline Allium ursinum & $\cdot$ & & · & . & . & . & & . & . & $\cdot$ & . & . & . & . & 3 & $\cdot$ & 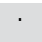 & + & 3 & 4 & 2 & 2 & + & + & + \\
\hline Cornus sanguinea & . & 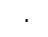 & . & 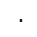 & . & . & . & . & . & . & . & . & . & + & 1 & . & 1 & + & + & + & . & 1 & . & . & . \\
\hline Viola suavis & . & . & . & & . & . & . & . & . & . & . & . & . & . & + & . & . & . & + & . & . & . & . & . & . \\
\hline Euonymus europaeus & . & 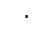 & . & 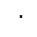 & . & . & . & . & . & . & . & . & . & 1 & . & . & . & . & . & . & . & . & . & . & . \\
\hline Bromus ramosus & . & . & . & 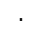 & . & . & & . & . & . & . & . & . & . & . & + & . & . & . & . & . & . & . & r & . \\
\hline Milium effusum & . & . & . & . & . & . & . & . & . & . & . & . & . & . & . & + & . & 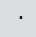 & . & . & . & . & . & . & . \\
\hline Ligustrum vulgare & . & & 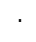 & & . & & . & . & . & $\cdot$ & . & . & . & . & . & $\cdot$ & & . & . & . & . & . & . & . & + \\
\hline \multicolumn{26}{|c|}{ Taxones característicos de asociación y unidades superiores } \\
\hline Polystichum setiferum & 1 & 1 & 1 & 3 & 1 & 1 & 1 & 1 & 1 & 1 & 2 & + & 1 & + & 2 & & 1 & 3 & 1 & 1 & 1 & 3 & 2 & 2 & + \\
\hline Viola riviniana & 1 & + & + & 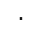 & + & + & 1 & 1 & 1 & 1 & 1 & 1 & 1 & 1 & 1 & + & 1 & 1 & 1 & + & + & 1 & 1 & 1 & 1 \\
\hline Athyrium filix-femina & 3 & 1 & + & 2 & 1 & 1 & 2 & 1 & 1 & + & 2 & 3 & . & 2 & 1 & $\cdot$ & 1 & 1 & 1 & + & 2 & 2 & 2 & 1 & 1 \\
\hline Dryopteris affinis & 2 & 1 & 1 & 1 & + & 1 & 1 & 1 & 1 & . & 1 & 1 & . & 1 & 1 & $r$ & + & 2 & + & . & 1 & 2 & 1 & 1 & . \\
\hline Brachypodium sylvaticum & 1 & & & + & 1 & 1 & + & + & 1 & 1 & & + & 2 & 1 & 2 & 1 & + & + & + & 1 & . & 1 & 2 & . & 2 \\
\hline Lonicera hispanica & . & 1 & 1 & . & + & 1 & 1 & 1 & 1 & $\cdot$ & 1 & 1 & + & 1 & 1 & + & 1 & 1 & 1 & + & 1 & . & 1 & 1 & . \\
\hline Primula acaulis & . & . & . & . & 1 & 1 & 1 & + & + & . & 2 & 1 & 1 & 2 & 1 & + & 2 & . & 1 & + & 1 & 2 & 1 & 1 & 1 \\
\hline Ranunculus ficaria & . & . & + & . & 1 & + & + & 1 & 1 & . & 1 & 1 & . & 1 & 1 & 1 & 1 & 1 & 2 & 1 & + & 2 & 1 & . & 1 \\
\hline Carex remota & + & + & & & 1 & 1 & 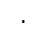 & + & 1 & 1 & + & + & 1 & 1 & 1 & . & + & + & & 1 & 1 & 1 & 2 & . & 2 \\
\hline Carex pendula & 2 & . & + & . & 2 & 2 & . & . & 1 & + & . & + & + & . & 1 & . & $\cdot$ & 3 & + & 1 & + & $\cdot$ & + & + & 1 \\
\hline Arum italicum & . & . & 1 & 1 & . & 1 & . & $\mathrm{r}$ & + & . & 1 & + & . & + & . & $\cdot$ & 1 & 1 & + & + & + & 2 & + & 1 & . \\
\hline Veronica montana & 1 & . & . & . & + & 1 & + & + & + & . & . & 1 & + & 1 & . & . & . & + & 1 & + & . & + & + & + & + \\
\hline Euphorbia dulcis & 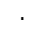 & & + & & 1 & & 2 & 2 & + & . & 3 & 1 & . & 1 & 1 & + & 1 & . & 1 & . & + & . & 1 & 1 & . \\
\hline Hypericum androsaemum & 1 & + & . & + & + & 1 & + & + & + & 1 & . & . & . & + & + & $\cdot$ & $\cdot$ & . & . & . & + & + & . & + & + \\
\hline Luzula sylvatica & 1 & + & 1 & . & . & . & 1 & 1 & 1 & . & . & . & . & . & 1 & 1 & 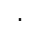 & . & 2 & + & 1 & . & 1 & 1 & 1 \\
\hline Potentilla sterilis & . & . & + & & 1 & + & + & . & 1 & . & 1 & . & . & + & 1 & $\cdot$ & 1 & 1 & 1 & . & + & + & + & . & . \\
\hline Carex sylvatica & . & + & . & & + & & + & . & 1 & . & 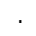 & . & + & . & + & + & & + & 1 & + & + & 1 & . & + & + \\
\hline Circaea lutetiana & + & . & . & . & + & . & + & . & . & . & . & + & . & 1 & + & . & + & + & + & . & . & . & + & . & + \\
\hline Melica uniflora & . & . & . & . & . & . & + & + & 1 & . & . & . & . & . & 1 & 1 & $\cdot$ & . & 2 & 2 & . & . & 1 & 1 & + \\
\hline Oxalis acetosella & . & 1 & + & 1 & 1 & 1 & 1 & + & + & + & . & . & . & . & . & . & . & . & . & . & + & . & . & . & . \\
\hline Blechnum spicant & + & + & + & . & 1 & 1 & + & 1 & . & + & . & . & . & . & . & $\cdot$ & . & . & . & . & + & + & . & . & . \\
\hline Symphytum tuberosum & . & . & 2 & 1 & 1 & 1 & . & . & + & + & . & . & . & 1 & . & . & . & . & 1 & . & . & . & . & . & . \\
\hline Phyllitis scolopendrium & 1 & 1 & 1 & 2 & + & . & . & . & . & . & 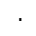 & . & . & . & . & . & 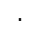 & . & . & . & + & + & . & . & . \\
\hline Stachys sylvatica & . & & . & 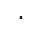 & . & . & . & . & + & . & 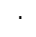 & 1 & . & + & . & 1 & . & . & 1 & . & . & 1 & $\cdot$ & . & 1 \\
\hline Ornithogalum pyrenaicum & . & & . & . & . & . & . & . & . & . & 1 & 3 & . & . & . & . & 1 & 1 & + & 1 & . & . & . & . & . \\
\hline Mercurialis perennis & . & & 2 & 1 & . & . & . & 1 & . & . & 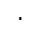 & . & . & . & . & 1 & & . & 1 & 1 & . & . & . & . & . \\
\hline
\end{tabular}




\begin{tabular}{|c|c|c|c|c|c|c|c|c|c|c|c|c|c|c|c|c|c|c|c|c|c|c|c|c|c|}
\hline $\mathrm{N}^{0}$ de orden & 1 & $2^{*}$ & $3^{*}$ & $4^{*}$ & 5 & 6 & 7 & 8 & 9 & 10 & 11 & 12 & 13 & 14 & 15 & 16 & 17 & 18 & 19 & 20 & 21 & 22 & 23 & 24 & 25 \\
\hline Lysimachia nemorum & 1 & $\cdot$ & $\cdot$ & $\cdot$ & + & 1 & + & + & $\cdot$ & + & . & $\cdot$ & . & $\cdot$ & $\cdot$ & $\cdot$ & $\cdot$ & $\cdot$ & $\cdot$ & $\cdot$ & $\cdot$ & $\cdot$ & . & $\cdot$ & . \\
\hline Solanum dulcamara & . & $\cdot$ & . & . & . & . & . & $\cdot$ & + & $\cdot$ & · & + & . & + & + & + & . & 1 & $\cdot$ & . & . & . & $\cdot$ & $\cdot$ & . \\
\hline Ranunculus tuberosus & . & $\cdot$ & + & . & . & + & + & 2 & . & . & . & . & . & $\cdot$ & . & . & . & . & + & . & . & . & $\cdot$ & $\cdot$ & . \\
\hline Senecio nemorensis & $\cdot$ & $\cdot$ & $\cdot$ & $\cdot$ & + & $\cdot$ & + & + & · & $\cdot$ & $\cdot$ & $\cdot$ & $\cdot$ & 1 & $\cdot$ & $\cdot$ & $\cdot$ & $\cdot$ & $\cdot$ & $\cdot$ & $\cdot$ & $\cdot$ & $\cdot$ & $\cdot$ & $\cdot$ \\
\hline \multicolumn{26}{|c|}{ Taxones característicos de Quercetea robori-petraeae y Carpino-Fagetea } \\
\hline Euphorbia amygdaloides & $\cdot$ & $\cdot$ & . & . & + & 1 & + & + & + & + & + & 1 & + & + & + & + & 1 & + & + & $\cdot$ & + & + & 1 & 1 & + \\
\hline Ajuga reptans & 1 & + & . & $\cdot$ & 1 & + & 1 & 1 & + & 1 & 1 & + & + & 1 & . & $\cdot$ & 1 & . & + & $\cdot$ & + & · & $\cdot$ & $\cdot$ & + \\
\hline Holcus mollis & . & + & 1 & + & + & . & . & $\cdot$ & + & . & + & $\cdot$ & 1 & + & 1 & + & . & 1 & . & $\cdot$ & + & . & . & $\cdot$ & 1 \\
\hline Hyacinthoides non-scripta & · & $\cdot$ & . & $\cdot$ & · & . & 1 & 1 & $\cdot$ & $\cdot$ & 1 & 2 & $\cdot$ & + & + & $\cdot$ & 3 & 1 & 1 & + & + & · & + & $\cdot$ & . \\
\hline Stellaria holostea & . & + & . & $\cdot$ & + & $\cdot$ & + & $\cdot$ & . & . & 2 & 2 & . & + & 1 & $\cdot$ & 1 & 1 & + & $\cdot$ & + & + & $\cdot$ & $\cdot$ & . \\
\hline Crepis lampsanoides & . & $\cdot$ & + & . & . & + & + & 1 & + & $\cdot$ & . & . & + & + & + & + & . & . & 1 & + & . & . & · & 1 & . \\
\hline Polypodium vulgare & $\cdot$ & $\cdot$ & . & $\cdot$ & · & + & + & + & + & · & . & $\cdot$ & + & + & 1 & $\cdot$ & . & 1 & + & + & + & · & $\cdot$ & + & . \\
\hline Dryopteris filix-mas & . & $\cdot$ & . & $\cdot$ & . & . & . & $\cdot$ & - & + & + & + & . & 1 & $\cdot$ & $\cdot$ & + & 2 & 1 & + & + & + & + & $\cdot$ & . \\
\hline Dryopteris dilatata & + & + & + & 1 & 1 & 1 & + & 1 & · & 1 & $\cdot$ & · & . & $\cdot$ & · & $\cdot$ & · & . & $\cdot$ & · & 1 & · & $\cdot$ & $\cdot$ & . \\
\hline Teucrium scorodonia & $\cdot$ & $\cdot$ & . & $\cdot$ & $\cdot$ & . & 1 & + & + & $\cdot$ & + & · & $\cdot$ & 1 & + & $\cdot$ & $\cdot$ & · & + & $\cdot$ & · & · & + & + & + \\
\hline Anemone nemorosa & . & $\cdot$ & . & . & + & + & 2 & 2 & · & . & . & . & $\cdot$ & $\cdot$ & 1 & $\cdot$ & + & . & 2 & + & . & $\cdot$ & 3 & $\cdot$ & . \\
\hline Aquilegia vulgaris & . & $\cdot$ & + & $\cdot$ & · & . & . & $\cdot$ & + & + & $\cdot$ & + & . & + & $\cdot$ & + & . & $\cdot$ & $\cdot$ & $\cdot$ & + & · & + & $\cdot$ & . \\
\hline
\end{tabular}

\begin{tabular}{|c|c|c|c|c|c|c|c|c|c|c|c|c|c|c|c|c|c|c|c|c|c|c|c|c|c|}
\hline Otros taxones & & & & & & & & & & & & & & & & & & & & & & & & & \\
\hline Rubus sp. & 2 & $\cdot$ & 1 & 1 & + & 1 & 1 & 1 & 1 & + & 1 & + & 2 & + & + & 1 & + & 1 & + & + & + & . & 1 & 1 & 1 \\
\hline Geum urbanum & + & . & . & + & + & + & . & + & 1 & 1 & 2 & 2 & 2 & 1 & 1 & + & 1 & 2 & 1 & + & . & 2 & 2 & 1 & 1 \\
\hline Geranium robertianum & 1 & 1 & + & 1 & + & . & . & + & + & . & 1 & 1 & + & 1 & + & 1 & + & + & + & + & + & 1 & . & 1 & 1 \\
\hline Brachypodium rupestre & . & . & + & . & + & . & 1 & 1 & 2 & . & 1 & 2 & . & 3 & 2 & . & 2 & 1 & 2 & . & 1 & 2 & + & 2 & 1 \\
\hline Heracleum sphondylium & + & . & $\cdot$ & . & 1 & . & . & + & + & . & 1 & $\cdot$ & 1 & 1 & 1 & + & 1 & 1 & + & + & . & 1 & + & + & + \\
\hline Silene dioica & 2 & 1 & + & 1 & 1 & 2 & + & + & 1 & + & . & . & . & 1 & + & 1 & . & . & + & . & . & 1 & . & + & . \\
\hline Alliaria petiolata & . & . & . & . & . & . & . & . & + & . & 1 & 2 & + & 1 & 1 & + & 1 & 2 & 1 & + & . & 1 & 1 & . & . \\
\hline Pteridum aquilinum & $\cdot$ & . & $\cdot$ & $\cdot$ & + & $r$ & + & . & 1 & $\cdot$ & 1 & + & 2 & + & + & $\cdot$ & + & + & + & $\cdot$ & + & . & . & . & . \\
\hline Ranunculus repens & + & + & . & + & + & + & . & . & + & + & + & . & 1 & + & . & 1 & . & . & . & . & . & . & . & + & . \\
\hline Eupatorium cannabinum & . & . & . & . & . & . & + & r & + & . & . & + & + & 1 & + & + & . & + & + & . & . & . & . & + & + \\
\hline Umbilicus rupestris & . & . & . & + & + & + & + & . & . & . & + & + & + & + & + & . & . & + & + & + & . & . & . & . & . \\
\hline Cardamine pratensis & 1 & + & . & . & + & 1 & + & . & . & + & . & · & + & . & . & . & + & . & + & . & . & + & + & . & . \\
\hline Pentaglottis sempervirens & $\cdot$ & $\cdot$ & . & $\cdot$ & $\cdot$ & $\cdot$ & . & . & + & . & 1 & 2 & $r$ & + & 1 & + & + & 1 & + & . & . & + & . & . & . \\
\hline Veronica chamaedrys & $\cdot$ & + & . & . & . & . & . & . & . & . & + & 1 & + & 1 & + & $\cdot$ & + & + & + & . & . & . & . & . & + \\
\hline Lapsana communis & . & . & . & . & . & . & . & . & . & . & + & 1 & + & 1 & 1 & . & . & + & + & . & . & . & + & 1 & . \\
\hline Dactylis glomerata & $\cdot$ & . & . & + & . & . & . & . & . & · & $\cdot$ & $\cdot$ & + & 1 & 1 & $\cdot$ & + & + & + & . & . & + & . & . & + \\
\hline Lamium maculatum & . & . & . & + & . & 1 & . & . & . & + & + & + & . & + & + & . & . & 1 & + & . & . & . & . & . & . \\
\hline Rumex acetosa & + & . & . & . & . & + & . & + & + & . & $\cdot$ & + & . & . & . & + & + & . & + & . & . & . & . & + & . \\
\hline Clematis vitalba & . & . & + & . & . & . & . & . & 1 & 1 & . & · & . & . & . & 1 & . & . & + & 1 & . & . & + & . & + \\
\hline Galium aparine & . & . & . & . & . & . & . & . & . & . & + & + & . & 1 & . & . & + & 1 & + & + & . & + & . & . & . \\
\hline Prunella vulgaris & . & . & . & . & . & . & + & + & + & . & + & . & + & 1 & . & + & . & . & . & . & . & . & . & . & + \\
\hline Scrophularia auriculata & + & + & . & . & . & . & . & . & . & + & . & . & . & . & + & + & . & + & . & . & . & + & . & + & . \\
\hline Equisetum arvense & $\cdot$ & . & . & . & + & . & . & . & 1 & . & . & . & + & + & . & 2 & . & . & . & + & . & . & . & + & . \\
\hline Centaurea rivularis & . & . & . & . & . & + & + & 1 & . & . & . & . & + & + & + & . & . & . & . & . & . & . & . & + & . \\
\hline Cardamine hirsuta & . & . & . & + & . & . & . & . & $r$ & . & $r$ & + & . & 1 & . & $\cdot$ & . & + & + & . & . & . & . & . & . \\
\hline Taraxacum gr. officinale & . & . & . & . & . & . & . & . & r & . & . & $r$ & . & r & . & . & . & + & + & . & . & . & . & + & r \\
\hline Lythrum salicaria & $\cdot$ & . & . & . & . & . & + & 1 & . & . & $\cdot$ & . & + & 1 & 1 & . & . & . & . & . & . & . & . & . & + \\
\hline Angelica sylvestris & 1 & . & + & . & . & + & . & + & . & + & . & . & . & . & . & 1 & . & . & . & . & . & . & . & . & . \\
\hline Chrysosplenium oppositifolium & + & . & + & 2 & . & 1 & . & . & . & . & . & . & . & . & . & $\cdot$ & . & . & . & . & + & . & . & . & . \\
\hline Limniris pseudacorus & + & . & . & $\cdot$ & $\cdot$ & $\cdot$ & . & . & . & . & . & 1 & + & + & + & $\cdot$ & . & . & . & . & . & . & . & . & . \\
\hline Vicia sepium & . & . & . & . & . & . & . & . & . & . & . & . & . & . & . & + & . & . & + & + & + & + & . & . & . \\
\hline Cystopteris fragilis & . & 1 & . & 1 & + & . & . & . & . & . & . & . & . & . & . & . & . & . & . & . & + & . & . & . & . \\
\hline Saponaria officinalis & . & . & . & . & . & . & . & . & . & . & . & . & . & + & . & + & 1 & . & + & . & . & . & . & . & . \\
\hline Asplenium trichomanes & $\cdot$ & + & + & 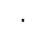 & $\cdot$ & . & . & . & . & . & $\cdot$ & . & . & . & . & $\cdot$ & . & . & . & . & + & . & . & + & . \\
\hline Picris hieracioides & . & + & . & . & . & . & + & + & . & . & . & . & + & . & . & . & . & . & . & . & . & . & . & . & . \\
\hline Fragaria vesca & $\cdot$ & . & ${ }^{\circ}$ & & + & . & . & . & + & . & . & + & + & . & . & . & . & . & . & . & . & . & . & . & . \\
\hline Polygonum sp. & . & . & . & . & . & . & . & . & . & . & . & . & + & + & + & . & . & + & . & . & . & . & . & . & . \\
\hline
\end{tabular}


Taxones presentes en menos de cuatro inventarios:

$\mathrm{E}_{1}+\mathrm{E}_{2}$ : Cytisus striatus: + en 14; Ligustrum ovalifolium: + en 5 y + en 14; Malus domestica: $r$ en 9; Malus sylvestris: 1 en 7 y 1 en 8 ; Prunus insititia: + en 20; Prunus padus: 1 en 16; Rosa arvensis: + en 23; Rosa micrantha: + en 9, 1 en 15 y + en 24; Rosa sp.: + en 8; Salix atrocinerea x S. fragilis: 2 en 16; Salix eleagnos: 1 en 16; Salix fragilis: 1 en 9, 1 en 14 y 1 en 16; Sorbus aucuparia: + en 16, Tilia platyphyllos: + en 16. Taxones característicos de asociación y unidades superiores: Allium scorzonerifolium: 1 en 8 ; Elymus caninus: 2 en 16; Moehringia trinervia: + en 15; Myosotis martini: 1 en 6 y + en 14, Potentilla montana: + en 7 y 1 en 8; Pulmonaria longifolia: + en 8 y + en 10. Taxones característicos de Quercetea robori-petraeae y Carpino-Fagetea: Doronicum plantagineum: 1 en 18; Lathyrus linifolius: + en 7 y + en 8; Poa nemoralis: 1 en 16 y + en 21; Stachys officinalis: 1 en 7 y 1 en 8 . Otros taxones: Aconitum neapolitanum: + en 16; Agrostis capillaris: 1 en 14 y 1 en 15; Agrostis stolonifera: + en 14; Alisma plantago-aquatica: + en 13 y r en 25; Anthoxanthum amarum: + en 7; Anthoxanthum odoratum:+ en 7; Apium nodiflorum: + en 14 y + en 18; Arrhenatherum bulbosum: 1 en 12 y + en 14; Artemisia absinthium: + en 16; Asplenium onopteris:+ en 9 y + en 21; Bidens sp.: + en 13, + en 14 y + en 15; Briza media: + en 15; Bryonia dioica: +en 11; Calamintha nepeta: + en 15; Calystegia sepium: 1 en 13 y 1 en 14; Canna indica: r en 8; Cardamine flexuosa: r en 9, + en 10 y r en 25; Carex cariophyllea: + en 7 y + en 8; Carex muricata: + en 14 y + en 15; Carex pilulifera: + en 7; Carum verticillatum: + en 8; Cerastium sp.: + en 14 y + en 19; Chelidonium majus: + en 17 y + en 18; Cirsium palustre: + en 16; Clinopodium vulgare: r en 2; Colchicum sp.: + en 19; Conyza bonariensis: + en 9 y + en 15; Cyperus eragrostis: + en 13; Digitalis purpurea: $r$ en 2, $r$ en 7 y + en 8; Epilobium sp.: + en 9, + en 13 y + en 14; Festuca gr. rubra: 1 en 8; Galeopsis tetrahit: + en 14, + en 16 y + en 23; Galium palustre: + en 14 y + en $15 ;$ Geranium lucidum: + en 13 y + en 19; Geranium purpureum: 1 en 15; Glechoma hederacea: 1 en 1, 2 en 14 y + en 19; Holcus lanatus: + en 13; Hydrangea macrophylla: $r$ en 2; Hypericum tetrapterum: + en 1; Hypericum undulatum: 1 en 15; Hypochoeris radicata: + en 15; Linaria triornithophora: $r$ en 15; Lotus corniculatus: + en 25; Lotus pedunculatus: + en 15; Lychnis flos-cuculi: + en 14; Lysimachia vulgaris: + en 13; Meconopsis cambrica: + en 16; Melissa officinalis: + en 13 y + en 18; Melissa officinalis: + en 13 y + en 18; Mentha aquatica: + en 1; Mentha longifolia: 1 en 16; Mentha suaveolens: + en 13; Molinia caerulea: + en 7 y 1 en 8; Narcissus bulbocodium: 1 en 8; Origanum virens: + en 24; Oxalis corniculata: r en 15; Phalaris arundinacea: 1 en 15, 1 en 16 y + en 25; Phytolacca americana: r en 10; Poa pratensis: + en 14; Polygonatum odoratum: + en 7 y + en 20; Polygonum hydropiper: + en 14; Polygonum persicaria: + en 1, + en 10 y + en 25; Polypodium cambricum: 1 en 3, 1 en 4 y + en 24; Potentilla erecta: + en 8; Prunella grandiflora: + en 7 y + en 14; Pseudoarrhenatherum longifolium: + en 7; Rubia peregrina: + en 7 y + en 10; Rumex conglomeratus: + en 15 y + en 18; Rumex obtusifolius: + en 16 y + en 25 ; Saxifraga granulata: + en 19; Senecio aquaticus: r en 8; Silene latifolia: + en 19; Sonchus oleraceus: + en 9, + en 15 y + en 23 ; Sparganium neglectum: + en 13 , + en 14 y + en 25; Stellaria alsine: + en 14; Stellaria media: + en 17, + en 19 y + en 20; Torilis arvensis: + en 15; Tradescantia fluminensis: + en 10; Trifolium pratense: + en 14 y + en 15; Tritonia x crocosmiflora: 1 en 7 y 1 en 8; Ulex europaeus: + en 7; Valeriana officinalis: 1 en 14 y + en 19; Veronica hederifolia: + en 19; Vinca sp.: + en 10; Vincetoxicum nigrum: + en 13; Vitis sp.: + en 10.

\section{Procedencia de los inventarios:}

1: As: Valdés, Río Llorio, margen izquierda por encima del puente de la carretera de El Pontigón a Ayones (709/4815); 2: Lu: Mondoñedo, Rego dos Casteláns, entre Curros y A Braña (634/4804); 3: As: El Franco, San Luís, Río do Mazo (675/4816); 4: Lu: Mondoñedo, Rego de Invernegas, entre Pousalido y O Vilar (631/4805); 5: C: Cariño, San Roque de Feás, Río de Landoi (588/4838); 6: Lu: Lourenzá, Santoadrao, Rego do Batán, aguas arriba de Lavandeira (640/4809); 7: C: Monfero/Cabanas, Río Eume, Cal grande, aguas arriba del puente colgante (572/4807); 8: C: Pontedeume, Ombre, A Alameda, margen izquierda del Río Eume, aguas abajo del área recreativa (571/4808); 9: Lu: Quiroga, Augas Mestas, Parada de Lor, Río Lor, aguas arriba de la estación de aforo (635/4702); 10: As: Villayón, Río Navia, entre la Presa de Arbón y la central eléctrica (683/4816); 11: Lu: Monforte de Lemos, A Vide, Río de Tuiriz (618/4709); 12: Lu: Sober, A Venda, Río das Neiras (618/4705); 13: Lu: Pantón, Río Cabe, aguas arriba de Ponte de Areas (612/4702); 14: Lu: O Páramo, A Veiga, Río Neira (620/4747); 15: Lu: Monforte, Ribas Altas, Río Cabe (624/4712); 16: Le: Villablino, Cuevas del Sil, Río Sil (712/4752); 17: Lu: Monforte de Lemos, Distriz, Río Cinsa (618/4707); 18: Lu: Monforte de Lemos, Canaval, margen izquierda del Río Cabe (615/4704); 19: Lu: Monforte de Lemos, entre A Parte y Remuín, margen derecha del Río Cabe, a altura de su unión con el Río Mao (625/4713); 20: Lu: Pobra de Brollón, A Veiga, Picais, Río Cabe (630/4717); 21: Lu: Quiroga, Campodola, Río Ferreiriño (646/4710); 22: Lu: Sarria, entre Rubián y Noceda, aguas abajo del Muiño da Ferrería (625/4725); 23: Lu: Quiroga, entre Sta. Cubicia y Lamela, Río de Quiroga (645/4705); 24: Lu: Pobra de Brollón, entre Barxa y A Ponte, margen derecha del Río Lor (636/4708); 25: Lu: Monforte de Lemos, As Barrioncas, Río Cabe (622/4712). 
Tabla VIII. Alisedas cantábricas occidentales "no termófilas" (Valeriano pyrenaicae-Alnetum glutinosae): 1-10: subvariante típica; 11: subvariante mesotrofa. Alisedas ovetenses oligo-mesotrofas (Hyperico androsaemi-Alnetum glutinosae) 12: variante de Osmunda regalis

\begin{tabular}{|c|c|c|c|c|c|c|c|c|c|c|c|c|}
\hline $\mathrm{N}^{\circ}$ de orden & 1 & 2 & 3 & 4 & 5 & 6 & 7 & 8 & 9 & 10 & 11 & 12 \\
\hline Altitud (m) & 545 & 470 & 525 & 550 & 350 & 480 & 635 & 1090 & 545 & 485 & 770 & 50 \\
\hline Pendiente $\left({ }^{\circ}\right)$ & 4 & 4 & 2 & 2 & 4 & 4 & $<2$ & $<2$ & 4 & $<2$ & 6 & $<2$ \\
\hline Orientación & $E$ & S & NE & NNW & ENE & E & NNW & SSE & SW & $E$ & NW & $\mathrm{E}$ \\
\hline Altura de copas (m) & 14 & 16 & 10 & 18 & 18 & 16 & 16 & 14 & 24 & 14 & 12 & 18 \\
\hline Cob. $E_{1}(\%)$ & 100 & 100 & 90 & 95 & 95 & 100 & 100 & 95 & 100 & 100 & 90 & 100 \\
\hline Cob. $E_{2}(\%)$ & 20 & 20 & 20 & 20 & 30 & 20 & 15 & 5 & 35 & 30 & 20 & 40 \\
\hline Cob. $E_{3}(\%)$ & 90 & 100 & 90 & 90 & 90 & 65 & 100 & 70 & 85 & 95 & 60 & 90 \\
\hline Área $\left(m^{2}\right)$ & 300 & 200 & 100 & 200 & 200 & 200 & 300 & 240 & 300 & 300 & 200 & 400 \\
\hline $\mathrm{N}^{0}$ de taxones & 34 & 33 & 40 & 41 & 31 & 45 & 43 & 37 & 53 & 44 & 35 & 59 \\
\hline \multicolumn{13}{|c|}{$E_{1}(>4,0 \mathrm{~m})+E_{2}(>1,5-4,0 \mathrm{~m}):$} \\
\hline Alnus glutinosa & 5 & 4 & 3 & 4 & 5 & 3 & 4 & 5 & 4 & 5 & 5 & 4 \\
\hline Salix atrocinerea & 1 & 2 & 1 & 1 & 1 & 3 & 1 & & 1 & + & 1 & 1 \\
\hline Corylus avellana & . & + & 1 & . & 2 & 2 & . & 1 & 3 & . & 2 & 1 \\
\hline Quercus robur & . & 1 & 1 & 1 & + & 1 & . & . & 1 & 1 & . & + \\
\hline Acer pseudoplatanus & . & . & $\cdot$ & 1 & . & 1 & . & 2 & 1 & 1 & 1 & 3 \\
\hline Sambucus nigra & 1 & 1 & $\cdot$ & 2 & . & . & 1 & . & + & + & . & 1 \\
\hline Fraxinus excelsior & . & . & $\cdot$ & 2 & . & 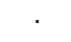 & 1 & . & 2 & + & . & 2 \\
\hline Ilex aquifolium & 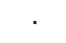 & . & . & & 1 & + & . & + & 1 & + & . & 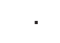 \\
\hline Castanea sativa & 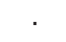 & + & . & . & . & - & . & . & 1 & . & + & + \\
\hline Crataegus monogyna & . & . & . & . & + & 1 & . & . & 1 & . & . & . \\
\hline Frangula alnus & + & 1 & + & . & . & . & . & . & . & . & . & . \\
\hline
\end{tabular}

$\mathrm{E}_{3}(<1,5 \mathrm{~m})$ :

Taxones diferenciales de Hyperico-Alnetum

Salix alba

Rosa sempervirens

Taxones diferenciales de subvariante de Osmunda regalis de Hyperico-Alnetum

\begin{tabular}{lllllllllll|l|l}
\hline Carex reuteriana & 1 & + & 2 & $\cdot$ & 1 & + & $\cdot$ & 1 & $\cdot$ & $\cdot$ & $\cdot$ & 1 \\
Osmunda regalis & $\cdot$ & $\cdot$ & $\cdot$ & $\cdot$ & $\cdot$ & $\cdot$ & $\cdot$ & $\cdot$ & $\cdot$ & $\cdot$ & $\cdot$ & +
\end{tabular}

\begin{tabular}{|c|c|c|c|c|c|c|c|c|c|c|c|}
\hline \multicolumn{12}{|c|}{ Taxones diferenciales de Valeriano-Alnetum frente a Hyperico-Alnetum } \\
\hline Saxifraga spathularis & 2 & . & 1 & 1 & 2 & 2 & . & + & 1 & 1 & + \\
\hline Betula pubescens & 1 & + & 2 & 1 & 1 & 2 & 1 & · & 1 & + & . \\
\hline Deschampsia subtriflora & 3 & 1 & 2 & . & 1 & + & + & . & . & + & . \\
\hline Viola palustris & 2 & 1 & 2 & 1 & . & 1 & . & . & . & . & . \\
\hline Omphalodes nitida & . & . & + & . & . & + & . & $r$ & + & . & + \\
\hline Erica arborea & - & + & + & . & - & + & + & . & . & . & . \\
\hline Vaccinium myrtillus & . & $r$ & + & . & - & + & $\cdot$ & . & . & . & + \\
\hline Peucedanum lancifolium & . & + & . & . & . & + & 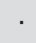 & . & . & · & . \\
\hline Allium victorialis & . & . & 1 & . & . & . & . & . & . & . & . \\
\hline Physospermum cornubiense & . & . & + & . & . & . & . & . & . & . & . \\
\hline Solidago virgaurea & . & . & . & . & + & . & . & . & . & . & . \\
\hline Carex laevigata & . & . & . & . & . & + & . & . & . & . & . \\
\hline Narcissus asturiensis & . & . & . & . & . & + & . & . & . & . & . \\
\hline Euphorbia hyberna & . & . & . & . & . & . & . & $r$ & . & . & . \\
\hline Caltha palustris & . & . & . & . & . & . & . & $r$ & . & . & . \\
\hline Ceratocapnos claviculata & . & · & . & - & · & · & . & . & · & . & r \\
\hline \multicolumn{12}{|c|}{ Taxones diferenciales de Valeriano-Alnetum frente a Senecioni-Alnetum } \\
\hline Chaerophyllum hirsutum & - & . & . & 2 & 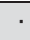 & . & 1 & 1 & . & 1 & + \\
\hline Adenostyles alpina & 1 & . & + & 1 & . & . & . & 1 & . & 1 & . \\
\hline Valeriana pyrenaica & . & . & . & 1 & + & . & + & . & 1 & . & . \\
\hline Scrophularia alpestris & . & . & + & 1 & . & . & . & . & . & 1 & . \\
\hline Lastrea limbosperma & . & 1 & 1 & + & . & . & 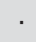 & . & . & . & . \\
\hline Avenella flexuosa & . & . & + & . & . & + & & . & . & . & . \\
\hline Dryopteris aemula & . & . & . & . & 1 & + & . & . & . & . & . \\
\hline Festuca gigantea & . & . & . & . & . & . & . & . & + & . & 1 \\
\hline Helleborus foetidus & . & . & . & · & . & · & & . & 1 & . & · \\
\hline Saxifraga lepismigena & . & . & . & . & & . & 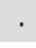 & + & - & . & - \\
\hline
\end{tabular}




\begin{tabular}{|c|c|c|c|c|c|c|c|c|c|c|c|c|}
\hline $\mathrm{N}^{\circ}$ de orden & 1 & 2 & 3 & 4 & 5 & 6 & 7 & 8 & 9 & 10 & 11 & 12 \\
\hline \multicolumn{13}{|c|}{ Taxones diferenciales de subvariante mesotrofa de Valeriano-Alnetum } \\
\hline Allium ursinum & $\cdot$ & $\cdot$ & $\cdot$ & $\cdot$ & $\cdot$ & $\cdot$ & $\cdot$ & $\cdot$ & $\cdot$ & 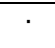 & + & $\cdot$ \\
\hline \multicolumn{13}{|c|}{ Taxones característicos de asociaciones y unidades superiores } \\
\hline Athyrium filix-femina & 2 & 3 & 1 & 4 & 1 & 1 & 2 & 3 & 1 & 4 & 2 & + \\
\hline Hedera hibernica & $\cdot$ & 1 & 2 & 1 & 3 & 1 & 1 & 1 & 2 & 2 & + & 3 \\
\hline Blechnum spicant & 1 & 3 & 1 & 1 & 1 & 2 & 1 & · & + & 1 & $r$ & . \\
\hline Viola riviniana & 1 & + & 1 & 1 & + & + & . & . & 1 & $\cdot$ & + & 2 \\
\hline Dryopteris affinis & 1 & 1 & 2 & 1 & . & 1 & 1 & . & 1 & 1 & 1 & . \\
\hline Lonicera hispanica & $\cdot$ & 1 & 1 & + & 1 & 1 & · & 1 & + & $\cdot$ & 1 & 1 \\
\hline Euphorbia dulcis & 1 & · & 1 & 1 & 1 & + & · & $r$ & + & $\cdot$ & 1 & . \\
\hline Primula acaulis & . & . & + & 1 & + & . & + & + & 1 & + & . & . \\
\hline Luzula sylvatica & 1 & . & 1 & · & 1 & 1 & . & 1 & . & $\cdot$ & 2 & . \\
\hline Senecio nemorensis & 1 & + & . & 1 & . & . & 1 & $\cdot$ & . & 1 & $\cdot$ & . \\
\hline Brachypodium sylvaticum & . & . & . & . & + & . & . & . & + & $\cdot$ & 1 & 1 \\
\hline Oxalis acetosella & . & . & . & + & . & 1 & . & + & + & . & . & . \\
\hline Hypericum androsaemum & . & . & . & . & + & . & . & . & + & + & . & + \\
\hline Polystichum setiferum & . & . & . & · & . & . & . & . & 2 & $\cdot$ & + & 2 \\
\hline Carex remota & . & . & . & . & . & + & . & . & 1 & $\cdot$ & $\cdot$ & + \\
\hline Lysimachia nemorum & $\cdot$ & . & . & . & . & + & · & $\cdot$ & · & 1 & $\cdot$ & · \\
\hline Stachys sylvatica & $\cdot$ & . & . & . & . & . & + & $\cdot$ & + & $\cdot$ & $\cdot$ & . \\
\hline Melica uniflora & . & . & . & . & · & . & · & + & + & $\cdot$ & $\cdot$ & . \\
\hline \multicolumn{13}{|c|}{ Taxones característicos de Quercetea robori-petraeae y Carpino-Fagetea } \\
\hline Teucrium scorodonia & 1 & + & 1 & 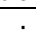 & + & + & + & + & + & 1 & + & $\cdot$ \\
\hline Holcus mollis & 1 & 1 & 1 & 1 & 1 & 1 & 2 & + & . & 1 & $\cdot$ & . \\
\hline Dryopteris dilatata & 1 & · & + & · & 1 & 1 & . & . & + & + & + & . \\
\hline Stellaria holostea & + & . & . & 1 & . & + & 1 & . & 1 & 1 & $\cdot$ & . \\
\hline Polypodium vulgare & $\cdot$ & + & + & + & . & . & + & + & . & $\cdot$ & $\cdot$ & . \\
\hline Crepis lampsanoides & . & . & . & . & . & + & . & + & . & . & + & + \\
\hline Euphorbia amygdaloides & . & . & . & . & . & . & . & + & + & 1 & . & + \\
\hline Anemone nemorosa & $\cdot$ & . & . & + & . & . & . & + & . & $\cdot$ & r & . \\
\hline Dryopteris filix-mas & . & . & . & · & . & . & + & + & + & $\cdot$ & . & . \\
\hline Ajuga reptans & . & . & . & . & . & . & . & . & 1 & $\cdot$ & 1 & . \\
\hline Aquilegia vulgaris & . & · & $\cdot$ & · & $\cdot$ & · & · & + & · & · & $r$ & · \\
\hline \multicolumn{13}{|l|}{ Otros taxones } \\
\hline Rubus sp. & 1 & 1 & 1 & 1 & + & 1 & 1 & 1 & 1 & + & 1 & 1 \\
\hline Brachypodium rupestre & 3 & 1 & 2 & . & 3 & 1 & . & . & . & 2 & . & 1 \\
\hline Dactylis glomerata & + & 1 & . & . & + & + & + & . & . & 1 & r & . \\
\hline Oenanthe crocata & 1 & . & 1 & 2 & . & . & 2 & . & . & 1 & . & 1 \\
\hline Agrostis capillaris & · & 1 & + & · & . & 1 & 1 & . & . & 1 & 1 & . \\
\hline Cruciata glabra & + & 1 & + & . & + & + & . & $\cdot$ & . & $\cdot$ & $\cdot$ & . \\
\hline Lamium maculatum & . & . & . & $r$ & . & . & 1 & . & + & + & . & + \\
\hline Silene dioica & . & . & . & 1 & . & . & . & . & 1 & 2 & . & + \\
\hline Geranium robertianum & . & . & . & + & . & . & 1 & . & . & + & + & . \\
\hline Ranunculus repens & + & · & . & + & . & . & + & $\cdot$ & . & + & $\cdot$ & . \\
\hline Rumex acetosa & + & · & . & . & . & $r$ & + & . & . & + & . & . \\
\hline Cardamine pratensis & 1 & . & . & + & . & + & . & . & . & $\cdot$ & . & . \\
\hline Centaurea rivularis & $\cdot$ & . & 1 & · & . & · & + & $\cdot$ & . & + & $\cdot$ & . \\
\hline Chrysosplenium oppositifolium & . & . & . & 1 & . & . & . & + & + & $\cdot$ & . & . \\
\hline Cardamine hirsuta & . & . & . & . & . & . & + & $\cdot$ & + & $\cdot$ & . & + \\
\hline Pentaglottis sempervirens & & . & . & . & . & . & + & $\cdot$ & + & $\cdot$ & $\cdot$ & + \\
\hline Epilobium sp. & . & . & . & . & . & . & + & $r$ & . & $\cdot$ & $\cdot$ & + \\
\hline
\end{tabular}


Taxones presentes solamente en 1 o 2 inventarios:

$\mathrm{E}_{1}+\mathrm{E}_{2}$ : Cornus sanguinea: 1 en 12; Cytisus scoparius: + en 6; Euonymus europaeus: 1 en 12; Ficus carica: 1 en $12 ;$ Juglans regia: + en 12; Laurus nobilis: 2 en 12; Ligustrum vulgare: 1 en 12; Populus nigra: 1 en 12; Prunus avium: 1 en 9 y + en 12; Prunus spinosa: + en 9; Rosa gr. canina: $r$ en 8. Taxones característicos de asociación y unidades superiores: Arum italicum: + en 12; Carex pendula: 1 en 12; Carex sylvatica: + en 9; Chamaeiris foetidissima: + en 12; Clematis vitalba: 1 en 12; Mercurialis perennis: 1 en 9; Myosotis martini: + en 10; Phyllitis scolopendrium: 1 en 12; Potentilla sterilis: + en 12; Pulmonaria longifolia: + en 9; Ranunculus ficaria: + en 9; Sanicula europea: $r$ en 11; Solanum dulcamara: + en 12; Veronica montana: $r$ en 8. Taxones característicos de Quercetea robori-petraeae y Carpino-Fagetea: Melampyrum pratense: $r$ en 8; Melittis melissophyllum: + en 9; Polygonatum verticillatum: + en 3; Stachys officinalis: 1 en 1; Tamus communis: 1 en 12. Otros taxones: Aconitum neapolitanum: 2 en 8; Alliaria petiolata: + en 12; Angelica major: + en 10; Anthoxanthum amarum: + en 4 y + en 10; Arrhenatherum bulbosum: + en 4; Asphodelus lusitanicus: + en 1; Calystegia sepium: + en 12; Carex pilulifera: + en 9; Cirsium palustre: + en 8; Conyza bonariensis: + en 12; Cystopteris fragilis: + en 4; Digitalis purpurea: + en 1 y + en 5; Equisetum arvense: + en 12; Geum urbanum: + en 7 y 1 en 9; Glechoma hederacea: + en 12; Heracleum sphondylium: + en 4 y 1 en 10; Holcus lanatus: + en 7; Hypericum tetrapterum: + en 7 y + en 11; Juncus effusus: + en 6; Lapsana communis: + en 1 y + en 7 ; Limniris pseudacorus: + en 12; Lotus corniculatus: + en 7; Lotus sp.: r en 11; Mentha aquatica: + en 12; Molinia caerulea: 2 en 3; Orobanche hederae: + en 12; Picris hieracioides: + en 4; Potentilla erecta: + en 2; Pteridum aquilinum: + en 2 y 1 en 10; Rubia peregrina: + en 12; Rumex obtusifolius: + en 7; Ruscus aculeatus: + en 12; Scrophularia auriculata: + en 1 y + en 7; Smilax aspera: + en 12; Taraxacum gr. officinale: $r$ en 12; Tritonia x crocosmiflora: $r$ en 12; Umbilicus rupestris: + en 2 y + en 7; Urtica dioica: 1 en 7 y 1 en 10; Veronica chamaedrys: + en 7 y + en 10; Vicia sepium: + en 9; Wahlenbergia hederacea: + en 8.

\section{Procedencia de los inventarios:}

1: Lu: Abadín, Río Pedrido, aguas arriba del puente entre Romariz y Os Agros (624/4808); 2: Lu: Abadín, Río Arnela, enfrente a Arnela (619/4801); 3: Lu: Abadín, Río Pedrido, aguas arriba de la confluencia con el Rego dos Agros (625/4809); 4: Lu: Abadín, Río Labrada, entre Labrada y As Basilias (621/4807); 5: Lu: Alfoz, Rego das Furnas, enfrente a AAbeleira (621/4815); 6: Lu: Quiroga, O Mazo de Soldón, Rego de Montouto (656/4711); 7: Lu: O Valadouro, Río das Furnas, Cadramón, aguas abajo del puente de la carretera de Abadín a Ferreira (619/4815); 8: Lu: Baleira, A Golpilleira, Rego do Lameirón (642/4766); 9: Le: Candín, Suertes, Río do Carballal (684/4748); 10: Lu: A Fonsagrada, Río Pontigón, entre Vilaframil e Vilardíaz (656/4786); 11: Lu: Abadín, As Goás, Río Labrada (622/4800); 12: As: Salas, entre Bárzana y Quintoños, Río Narcea (730/4808). 